\title{
Correlation of Photocatalytic Activity with Band Structure of Low- dimensional Semiconductor Nanostructures
}

\author{
Fanke Meng \\ West Virginia University
}

Follow this and additional works at: https://researchrepository.wvu.edu/etd

\section{Recommended Citation}

Meng, Fanke, "Correlation of Photocatalytic Activity with Band Structure of Low-dimensional Semiconductor Nanostructures" (2013). Graduate Theses, Dissertations, and Problem Reports. 468. https://researchrepository.wvu.edu/etd/468

This Dissertation is protected by copyright and/or related rights. It has been brought to you by the The Research Repository @ WVU with permission from the rights-holder(s). You are free to use this Dissertation in any way that is permitted by the copyright and related rights legislation that applies to your use. For other uses you must obtain permission from the rights-holder(s) directly, unless additional rights are indicated by a Creative Commons license in the record and/ or on the work itself. This Dissertation has been accepted for inclusion in WVU Graduate Theses, Dissertations, and Problem Reports collection by an authorized administrator of The Research Repository @ WVU. For more information, please contact researchrepository@mail.wvu.edu. 


\title{
Correlation of Photocatalytic Activity with Band Structure of Low-dimensional Semiconductor Nanostructures
}

\author{
Fanke Meng \\ Dissertation submitted to the \\ Benjamin M. Statler College of Engineering and Mineral Resources \\ at West Virginia University
}

in partial fulfillment of the requirements for the degree of

Doctor of Philosophy

In

Mechanical Engineering

Nianqiang Wu, Ph.D., Chair

Ever J. Barbero, Ph.D.

Lian-Shin Lin, Ph.D.

Dongling Ma, Ph.D.

Terence Musho, Ph.D.

Department of Mechanical and Aerospace Engineering

Morgantown, West Virginia University

2013

Keywords: $\mathbf{H}_{2}$, low-dimensional, nanostructure, photocatalysis, semiconductor, solar

Copyright 2013 [Fanke Meng] 


\section{ABSTRACT \\ Correlation of Photocatalytic Activity with Band Structure of Low-dimensional Semiconductor Nanostructures}

Fanke Meng

Photocatalytic hydrogen generation by water splitting is a promising technique to produce clean and renewable solar fuel. The development of effective semiconductor photocatalysts to obtain efficient photocatalytic activity is the key objective. However, two critical reasons prevent wide applications of semiconductor photocatalysts: low light usage efficiency and high rates of charge recombination. In this dissertation, several lowdimensional semiconductors were synthesized with hydrothermal, hydrolysis, and chemical impregnation methods. The band structures of the low-dimensional semiconductor materials were engineered to overcome the above mentioned two shortcomings. In addition, the correlation between the photocatalytic activity of the lowdimensional semiconductor materials and their band structures were studied.

First, we studied the effect of oxygen vacancies on the photocatalytic activity of one-dimensional anatase $\mathrm{TiO}_{2}$ nanobelts. Given that the oxygen vacancy plays a significant role in band structure and photocatalytic performance of semiconductors, oxygen vacancies were introduced into the anatase $\mathrm{TiO}_{2}$ nanobelts during reduction in $\mathrm{H}_{2}$ at high temperature. The oxygen vacancies of the $\mathrm{TiO}_{2}$ nanobelts boosted visible-lightresponsive photocatalytic activity but weakened ultraviolet-light-responsive photocatalytic activity. As oxygen vacancies are commonly introduced by dopants, these results give insight into why doping is not always beneficial to the overall photocatalytic performance despite increases in absorption. Second, we improved the photocatalytic performance of two-dimensional lanthanum titanate $\left(\mathrm{La}_{2} \mathrm{Ti}_{2} \mathrm{O}_{7}\right)$ nanosheets, which are widely studied as an efficient photocatalyst due to the unique layered crystal structure. Nitrogen was doped into the $\mathrm{La}_{2} \mathrm{Ti}_{2} \mathrm{O}_{7}$ nanosheets and then $\mathrm{Pt}$ nanoparticles were loaded onto the $\mathrm{La}_{2} \mathrm{Ti}_{2} \mathrm{O}_{7}$ nanosheets. Doping nitrogen narrowed the band gap of the $\mathrm{La}_{2} \mathrm{Ti}_{2} \mathrm{O}_{7}$ nanosheets by introducing a continuum of states by the valence band edge, unlike the mid-gap states introduced by oxygen vacancies, leading to an improvement in visible and 
UV photocatalysis. The Pt nanoparticles both enhanced separation of charge carriers and acted as reaction sites for hydrogen evolution. The photocatalytic hydrogen generation rate of the $\mathrm{La}_{2} \mathrm{Ti}_{2} \mathrm{O}_{7}$ nanosheets was increased to $\sim 21 \mathrm{\mu} \mathrm{M} \mathrm{g}^{-1} \mathrm{hr}^{-1}$ from zero in visible light by nitrogen doping and Pt loading, showing the importance of the positioning of dopant energy levels within the band gap.

Third, a hematite/reduced graphene oxide $\left(\alpha-\mathrm{Fe}_{2} \mathrm{O}_{3} / \mathrm{rGO}\right)$ nanocomposite was synthesized by a hydrolysis method. The photocatalytic oxygen evolution rate of the hematite was increased from 387 to $752 \mathrm{\mu} \mathrm{M} \mathrm{g}^{-1} \mathrm{hr}^{-1}$ by incorporating rGO. Photoelectrochemical measurements showed that coupling the hematite nanoparticles with the rGO can greatly increase the photocurrent and reduce the charge recombination rate, overcoming the poor charge recombination characteristics of hematite and allowing its small band gap to be taken advantage of. Fourth, a $\mathrm{Au} / \mathrm{La}_{2} \mathrm{Ti}_{2} \mathrm{O}_{7} / \mathrm{rGO}$ heterostructure was synthesized to further enhance the photocatalytic hydrogen generation rate of the $\mathrm{La}_{2} \mathrm{Ti}_{2} \mathrm{O}_{7}$ nanosheets. The enhanced performance of photocatalytic water splitting was due to plasmonic energy transfer, which resulted from the plasmonic Au nanoparticles on the $\mathrm{La}_{2} \mathrm{Ti}_{2} \mathrm{O}_{7}$ nanosheets. This heterostructure showed doping, charge extraction, and plasmonics work synergistically. Fifth, nanoscale $\mathrm{p}-\mathrm{n}$ junctions on the rGO were formed by depositing the $p$-type $\mathrm{MoS}_{2}$ nanoplatelets onto the $n$-type nitrogen-doped rGO. The $p$ $\mathrm{MoS}_{2} / n$-rGO heterostructure had significant photocatalytic hydrogen generation activity under solar light irradiation. The enhanced charge generation and suppressed charge recombination due to the $\mathrm{p}-\mathrm{n}$ junctions led to enhance solar hydrogen generation reaction while allowing replacement of the expensive Pt nanoparticles with an eco-friendly alternative.

The research results in this dissertation are contributed to a better understanding of the relationship between the band structure tuning and photocatalytic activity of lowdimensional semiconductor nanostructures. The results lay out guidelines for the enhancement of large band gap semiconductors with poor solar utilization and small band gap semiconductors with poor charge recombination characteristics alike. Additionally, it is shown that the rare earth co-catalyst can be replaced with an earth friendly alternative, 
leading to a further increase in performance. The findings of this thesis can be used to guide photocatalyst selection and optimization for solar to hydrogen conversion. 


\section{ACKNOWLEDGEMENTS}

I greatly thank my supervisor Associate Professor Nianqiang (Nick) Wu. I am grateful for his valuable suggestions and outstanding guidance in my projects and my 5year studies for Ph.D degree in West Virginia University.

I would like to thank Prof. Ever J. Barbero, Prof. Lian-Shin Lin, Prof. Dongling Ma and Prof. Terence Musho as my Ph.D committees to give me sincere direct and timely advice.

I am thankful to Dr. Ayyakkannu Manivannan, Dr. Weiqiang Ding, Dr. James Poston for their support of materials characterizations with BET, XPS and XRD.

Special thanks are due to Dr. Jin Wang, Dr. Mingjia Zhi, Dr. Ming Li, Dr. Jiangtian Li, Dr. Honglei Gou, Chengcheng Xiang, Scott Cushing, Savan Suri, Joey Bright, Peng Zheng for their inspiring discussion during my Ph. D study.

This work was financially supported by the West Virginia University Research Corporation and the West Virginia EPSCoR Office; the Research Challenge Grant of West Virginia State (EPS08-01); the State Key Laboratory of Silicon Materials (Grant No. SKL2009-14) at Zhejiang University, China. It was also partially financial support by National Science Foundation (NSF) grant (CBET-1033266) and NSF RII grant (EPS 1003907). 


\section{Table of Contents}

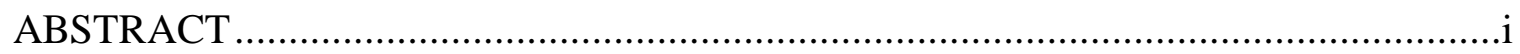

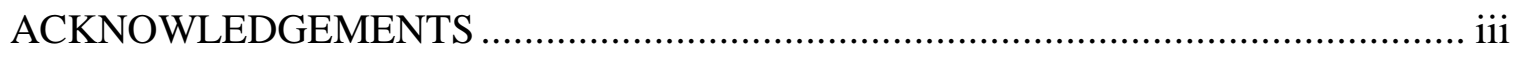

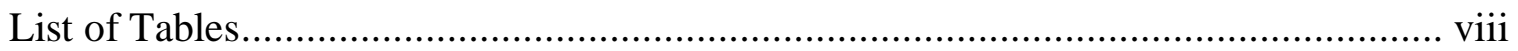

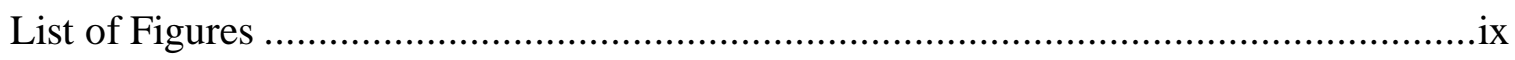

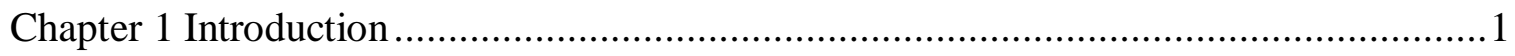

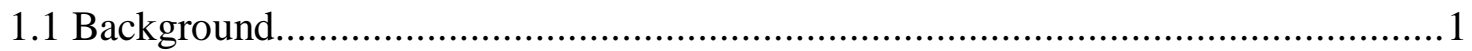

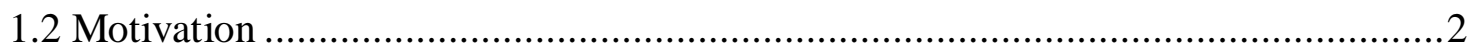

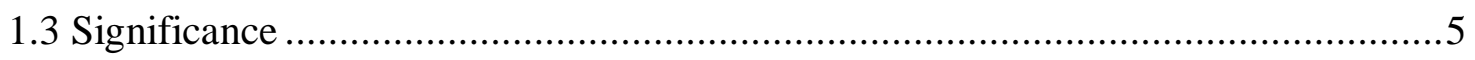

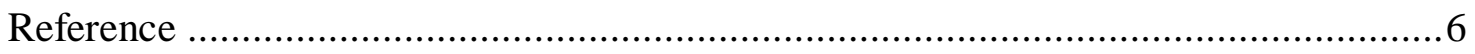

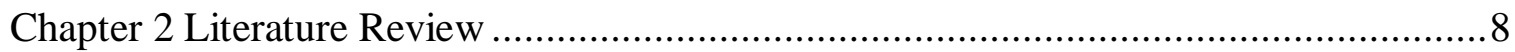

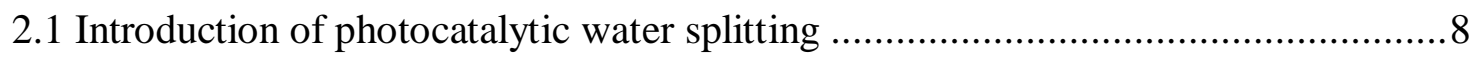

2.1.1 Processes of photocatalytic water splitting ....................................................

2.1.2 Fundamental theory of photocatalytic water splitting .....................................11

2.1.3 Evaluation of photocatalytic water splitting ...............................................14

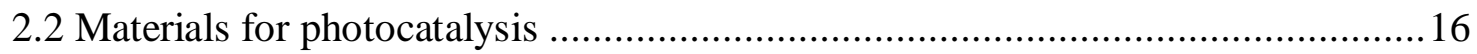

2.3 Approaches for improvement of photocatalytic activity ......................................18

2.3.1 Metal/Nonmetal ion doping and codoping ................................................ 18

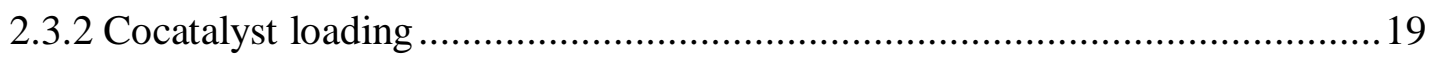

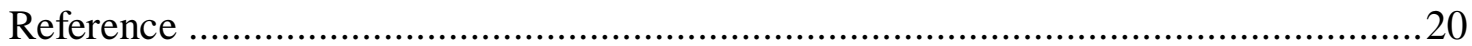

Chapter 3 Effects of Oxygen Vacancy to the Photocatalytic Activity of Anatase $\mathrm{TiO}_{2} \ldots 26$

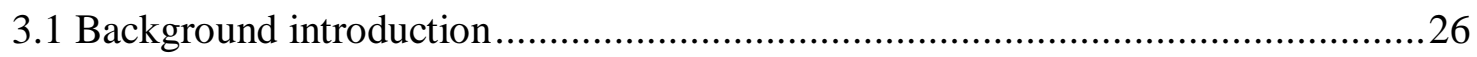

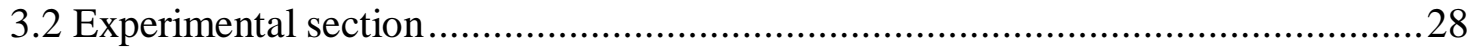

3.2.1 Synthesis of the pristine and reduced $\mathrm{TiO}_{2}$ nanobelts..................................28

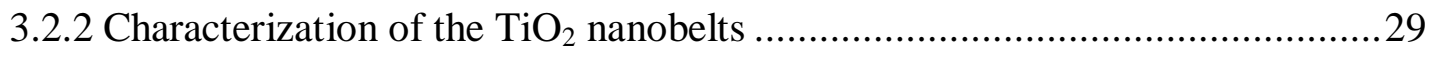

3.2.3 Evaluation of photocatalytic activity of the $\mathrm{TiO}_{2}$ nanobelts............................30

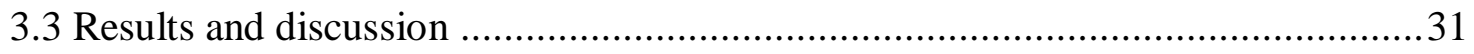

3.3.1 Morphology and crystal structure of the $\mathrm{TiO}_{2}$ nanobelts ...............................31

3.3.2 Chemical status of the $\mathrm{Ti}$ and $\mathrm{O}$ in the $\mathrm{TiO}_{2}$ nanobelts ..................................32

3.3.3 Light absorption of the $\mathrm{TiO}_{2}$ nanobelts .........................................................34 


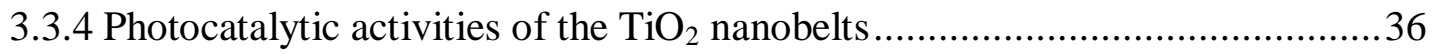

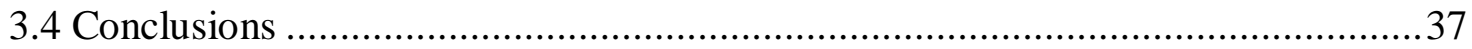

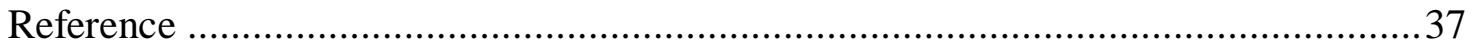

Chapter 4 Visible Light Photocatalytic Activity of Nitrogen-Doped $\mathrm{La}_{2} \mathrm{Ti}_{2} \mathrm{O}_{7}$ Nanosheets Originating from Band Gap Narrowing ............................................................ 41

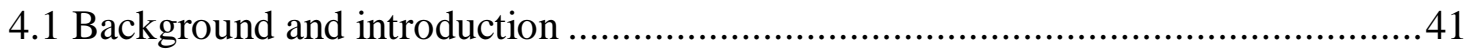

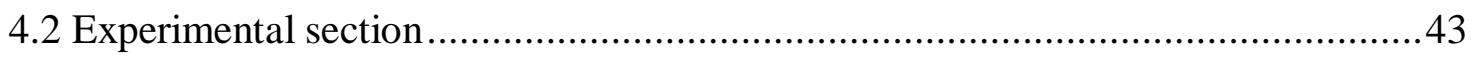

4.2.1 Chemicals and materials ................................................................. 43

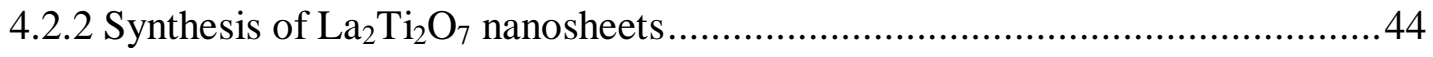

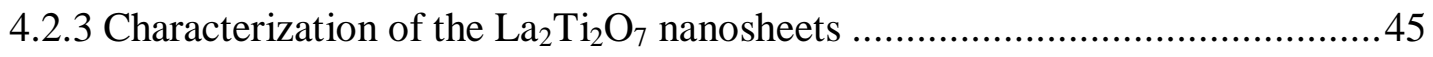

4.2.4 Photocatalysis testing of the $\mathrm{La}_{2} \mathrm{Ti}_{2} \mathrm{O}_{7}$ nanosheets ...............................46

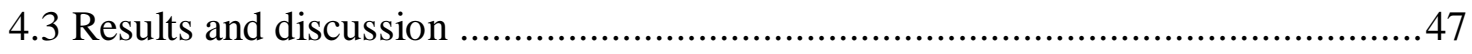

4.3.1 Morphology and crystal structure ............................................... 47

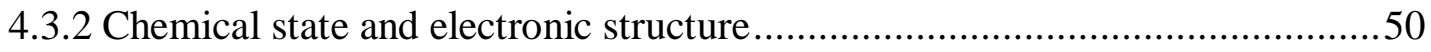

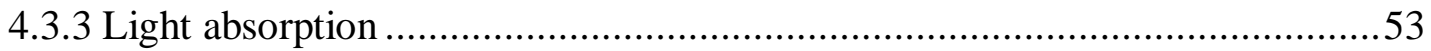

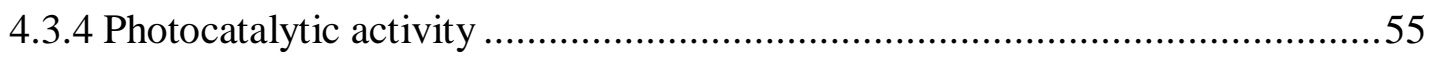

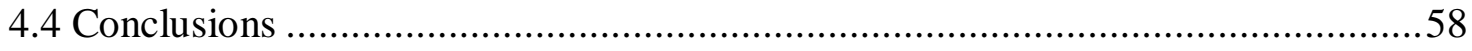

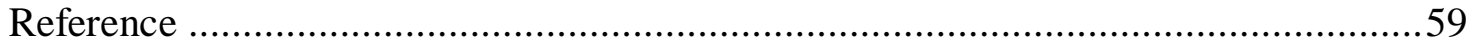

Chapter 5 Photocatalytic Generation of Hydrogen with Visible-light Nitrogen-doped

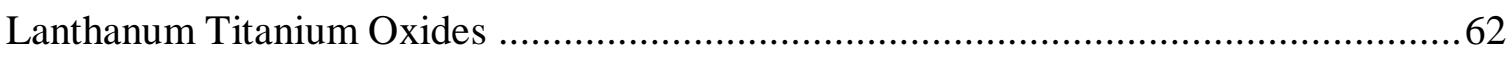

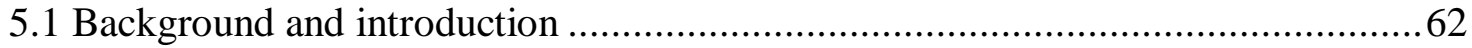

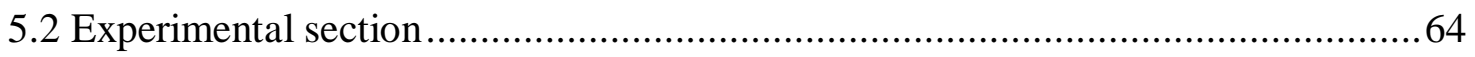

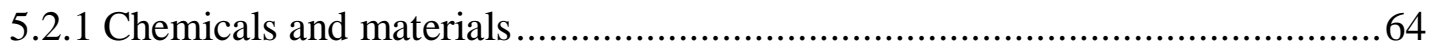

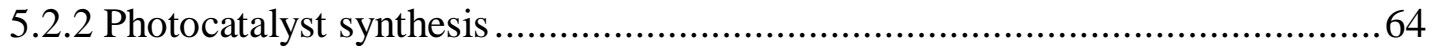

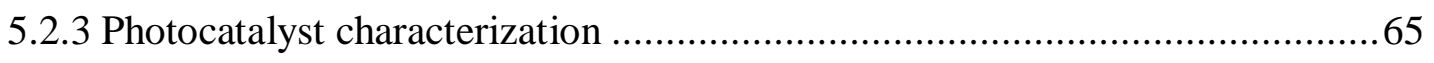

5.2.4 Hydrogen generation by photocatalytic water splitting ............................66

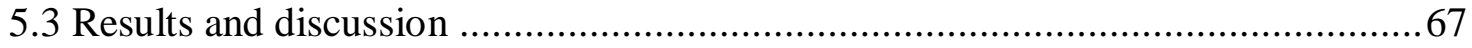

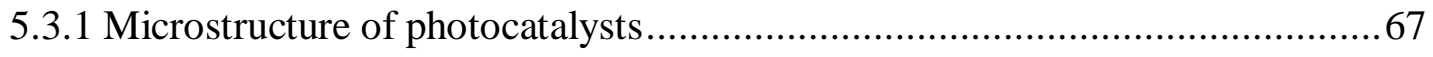

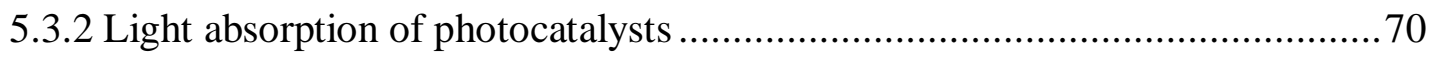

5.3.3 Photocatalytic hydrogen generation ................................................. 71

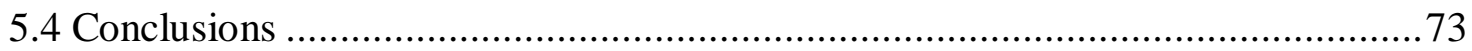


Chapter 6 Photocatalytic Water Oxidation by Hematite/Reduced Graphene Oxide Composite .76

6.1 Background and introduction ................................................................ 76

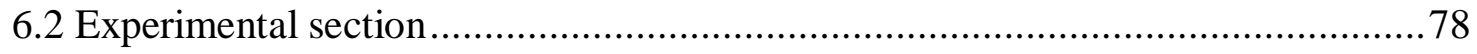

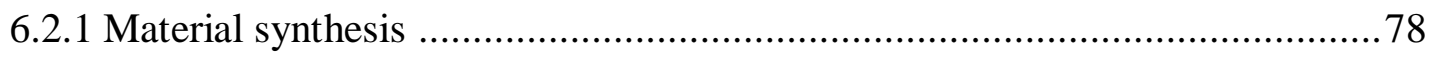

6.2.2 Characterizations and light absorption measurement ............................... 79

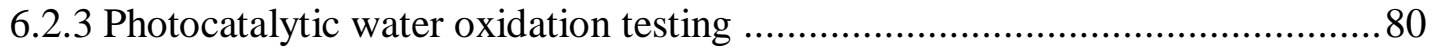

6.2.4 Photoelectrochemical measurement ................................................. 81

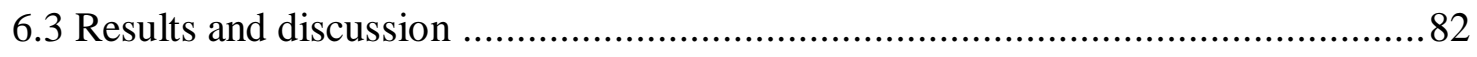

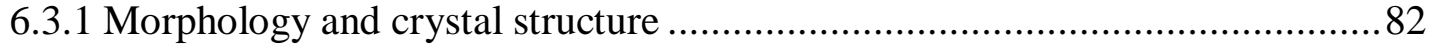

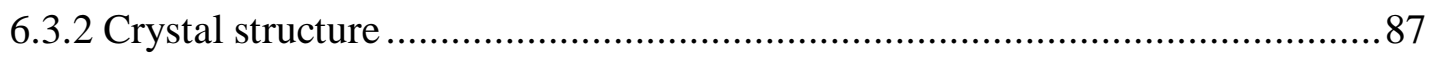

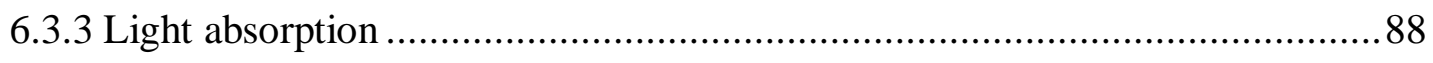

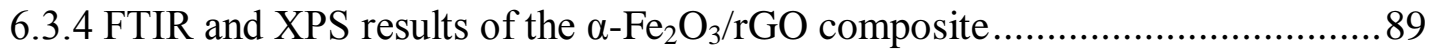

6.3.5 Photocatalytic oxygen generation ............................................... 91

6.3.6 Photoelectrochemical measurements .................................................93

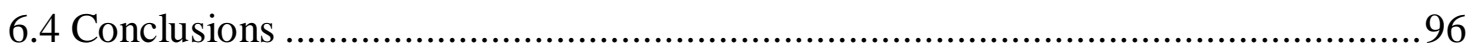

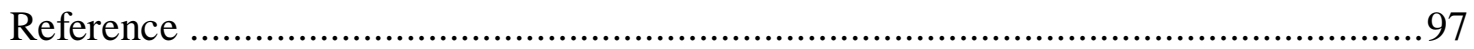

Chapter 7 Solar $\mathrm{H}_{2}$ Generation of $\mathrm{Au} / \mathrm{La}_{2} \mathrm{Ti}_{2} \mathrm{O}_{7} / \mathrm{rGO}$ Heterostructure Enhancement by

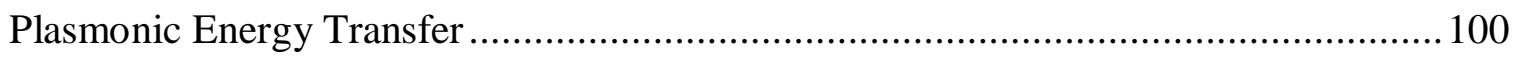

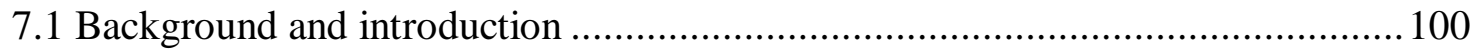

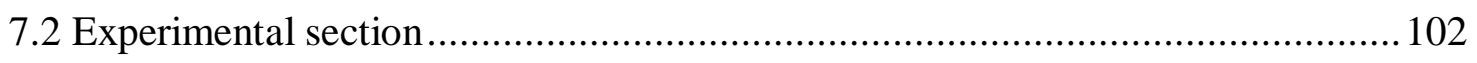

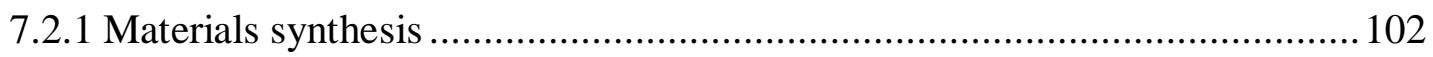

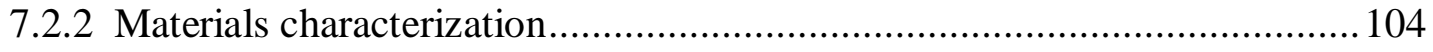

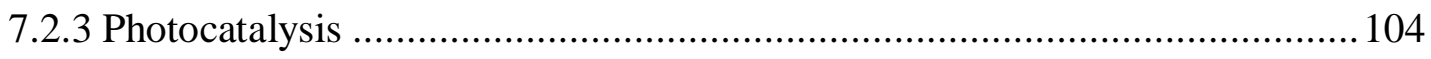

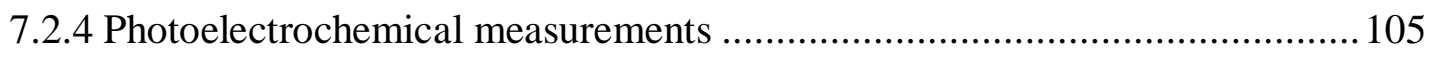

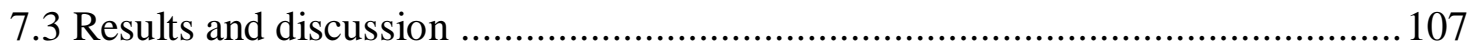

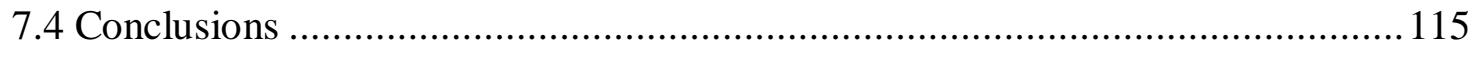

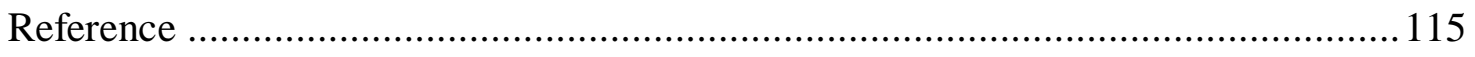

Chapter 8 Solar Hydrogen Generation by Nanoscale p-n Junction of p-type Molybdenum Disulfide/n-type Nitrogen-Doped Reduced Graphene Oxide. 


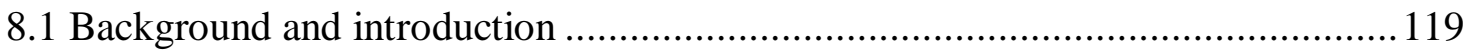

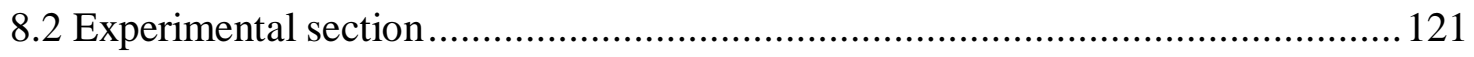

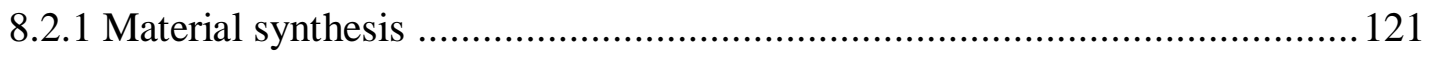

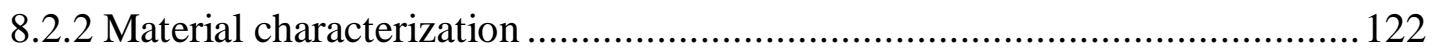

8.2.3 Photocatalytic $\mathrm{H}_{2}$ generation ........................................................... 123

8.2.4 Photoelectrode fabrication and photoelectrochemical measurement ............ 124

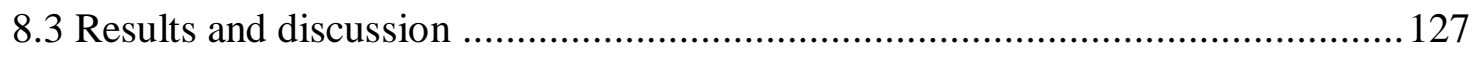

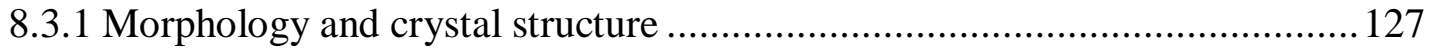

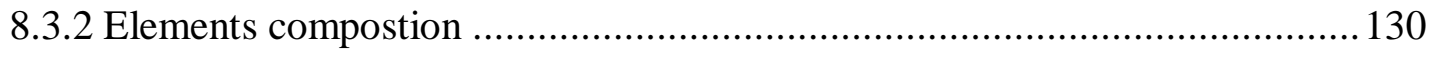

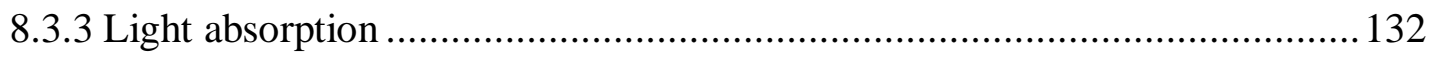

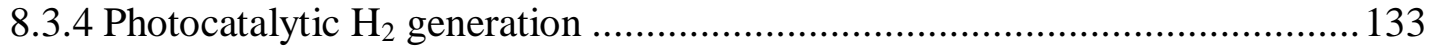

8.3.5 Photoelectrochemical measurements ..................................................... 135

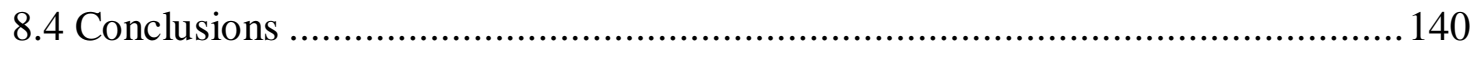

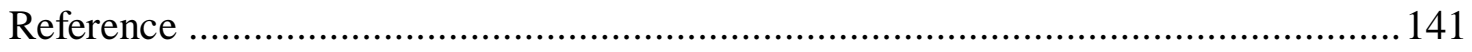

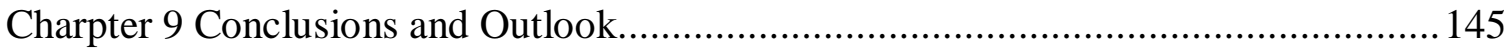

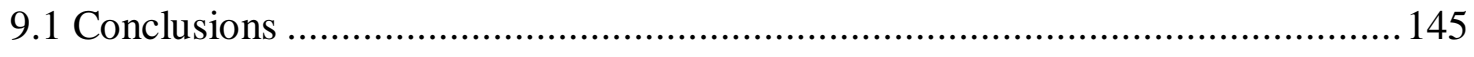

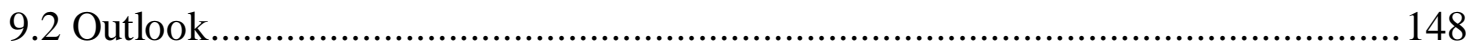

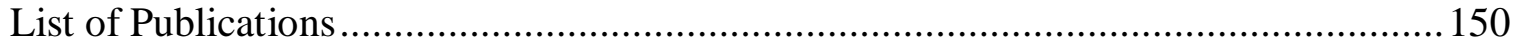




\section{List of Tables}

Page

\#

Table 7-1 The contents of the loaded Pt and Au and doped $\mathrm{N}$ in the four $\mathbf{1 0 9}$ samples.

Table 7-2 The photocatalytic hydrogen generation rates and BET surface 114 areas of the four samples.

Table 8-1 The intensity of sun light during photocatalysis tests 134

Table 8-2 Normalized $\mathrm{H}_{2}$ generation rates of the $\mathrm{p}-\mathrm{MoS}_{2} / \mathrm{n}-\mathrm{rGO}$ under $\mathbf{1 3 5}$ sunlight irradiation 


\section{List of Figures}

Figure 2-1 Schematic of energy change in photosynthesis and photocatalytic $\quad \mathbf{8}$ water splitting [1]

Figure 2-2 Schematic of different processes of photocatalytic water splitting

9 [1]

Figure 2-3 Schematic of principle of water splitting using semiconductor photocatalysts [1]

Figure 2-4 $\mathrm{pH}$ dependence of the conduction band and valence band in an aqueous electrolyte solution [4]

Figure 2-5 Schematic of half reactions of water splitting $-\mathrm{H}_{2}$ or $\mathrm{O}_{2}$ evolution reactions

Figure 3-1 Light spectra of (a) LZC-UVA, Luzchem lamps and (b) LZC420, Luzchem lamps

Figure 3-2 (a) SEM image of pristine $\mathrm{TiO}_{2}$ nanobelts; (b) XRD patterns of pristine and reduced $\mathrm{TiO}_{2}$ nanobelts

Figure 3-3 XPS spectra of $\mathrm{Ti} 2 \mathrm{p}$ peaks of pristine $\mathrm{TiO}_{2}$ nanobelts (a) and reduced $\mathrm{TiO}_{2}$ nanobelts (b)

Figure 3-4 XPS spectra of $\mathrm{O}$ 1s peaks of pristine $\mathrm{TiO}_{2}$ nanobelts (a) and reduced $\mathrm{TiO}_{2}$ nanobelts (b)

Figure 3-5 Diffuse-reflectance spectra of the pristine $\mathrm{TiO}_{2}$ nanobelts and the reduced $\mathrm{TiO}_{2}$ nanobelts. The insets are the optical photos of the pristine $\mathrm{TiO}_{2}$ (left) and reduced $\mathrm{TiO}_{2}$ (right), which shows the color change from white to blue due to reduction in $\mathrm{H}_{2}$ flow at $600{ }^{\circ} \mathrm{C}$

Figure 3-6 Photocatalytic activities of the pristine $\mathrm{TiO}_{2}$ nanobelts $\left(\mathrm{P}-\mathrm{TiO}_{2}\right)$ and the reduced $\mathrm{TiO}_{2}\left(\mathrm{R}-\mathrm{TiO}_{2}\right)$ under visible light irradiation (a), and under UV light irradiation (b)

Figure 4-1 Energy-dispersive $\mathrm{X}$-ray spectroscopy (EDX) spectrum obtained from the pristine $\mathrm{La}_{2} \mathrm{Ti}_{2} \mathrm{O}_{7}$ after hydrothermal processing

Figure 4-2 Images of the pristine $\mathrm{La}_{2} \mathrm{Ti}_{2} \mathrm{O}_{7}$ nanosheets including SEM images at low (a) and high (b) magnification, AFM images in 
two-dimension (c), three-dimensions (d) and height profile analysis (e) along the green line in (c), XRD patterns obtained before and after nitrogen doping (f), and a HRTEM image (g).

Figure 4-3 Schematic of perovskite structure of $\mathrm{La}_{2} \mathrm{Ti}_{2} \mathrm{O}_{7} \quad 48$

Figure 4-4 SEM image of the nitrogen-doped La2Ti2O7 nanosheets 49

Figure 4-5 XPS spectra obtained from the pristine and the nitrogendoped $\mathrm{La}_{2} \mathrm{Ti}_{2} \mathrm{O}_{7}$ nanosheets showing the $\mathrm{N} 1 \mathrm{~s}$ core level (a), and the valence band (b).

Figure 4-6 XPS spectra of the Ti $2 p$ core-level of the pristine (a) and the nitrogen-doped (b) $\mathrm{La}_{2} \mathrm{Ti}_{2} \mathrm{O}_{7}$ nanosheets.

Figure 4-7 XPS spectra of the La 3d core-level of the pristine and nitrogendoped $\mathrm{La}_{2} \mathrm{Ti}_{2} \mathrm{O}_{7}$

Figure 4-8 Diffuse reflectance spectra of the pristine and nitrogendoped $\mathrm{La}_{2} \mathrm{Ti}_{2} \mathrm{O}_{7}$ nanosheets. The insets are the optical photos of the nanosheet samples, showing the change in the sample color from white (left) to yellow (right) due to nitrogen-doping.

Figure 4-9 Schematic illustration of the effects of nitrogen-doping on the band structures of pristine $\mathrm{La}_{2} \mathrm{Ti}_{2} \mathrm{O}_{7}$ (a), and anatase $\mathrm{TiO}_{2}(\mathrm{~b})$.

Figure 4-10 Photocatalytic activities of the pristine and the nitrogendoped $\mathrm{La}_{2} \mathrm{Ti}_{2} \mathrm{O}_{7}$ nanosheets under visible light irradiation (a), and under ultraviolet light irradiation (b).

Figure 5-1 (a) SEM image of the pristine $\mathrm{La}_{2} \mathrm{Ti}_{2} \mathrm{O}_{7}$ nanosheets; (b) TEM image of the Pt-loaded nitrogen-doped $\mathrm{La}_{2} \mathrm{Ti}_{2} \mathrm{O}_{7}$ nanosheets; (c) HRTEM image of the Pt-loaded nitrogendoped nanosheets and (d) XRD patterns of the pristine and the Pt-loaded nitrogen-doped nanosheets.

Figure 5-2 EDX spectrum obtained from the nitrogen doped $\mathrm{La}_{2} \mathrm{Ti}_{2} \mathrm{O}_{7}$ nanosheets loaded with the Pt nanoparticles.

Figure 5-3 SEM image of the nitrogen-doped $\mathrm{La}_{2} \mathrm{Ti}_{2} \mathrm{O}_{7}$ nanosheets. 68

Figure 5-4 XPS spectra of N 1s (a) and Pt 4f (b) of the nitrogen-doped $\mathrm{La}_{2} \mathrm{Ti}_{2} \mathrm{O}_{7}$ nanosheets loaded with Pt.

Figure 5-5 UV-vis absorption spectra of the pristine $\mathrm{La}_{2} \mathrm{Ti}_{2} \mathrm{O}_{7}$ nanosheets (a), the nitrogen-doped nanosheets (b), and the Pt-loaded 
nitrogen-doped nanosheets (c).

Figure 5-6 Hydrogen production by four samples under the visible light (a) 72 and the ultraviolet (b) irradiation.

$\begin{array}{lll}\text { Figure 5-7 Schematic of photocatalytic hydrogen generation. } & \mathbf{7 2}\end{array}$

Figure 6-1 SEM image of the $\alpha-\mathrm{Fe}_{2} \mathrm{O}_{3} / \mathrm{rGO}(\mathrm{h})$ composite containing 6.1 $\mathrm{wt} \%$ of reduced graphene oxide ( $\mathrm{rGO}$ ) after heat treatment at $800{ }^{\circ} \mathrm{C}$

Figure 6-2 (a) SEM and (b) TEM images of the $\alpha-\mathrm{Fe}_{2} \mathrm{O}_{3} / \mathrm{rGO}$

Figure 6-3 SEM image of the $\alpha-\mathrm{Fe}_{2} \mathrm{O}_{3} / \mathrm{rGO}(\mathrm{l})$ composite containing $2.9 \mathrm{wt} \%$ of reduced graphene oxide (rGO) after heat treatment at $800{ }^{\circ} \mathrm{C}$

Figure 6-4 SEM image of pristine $\alpha-\mathrm{Fe}_{2} \mathrm{O}_{3}$; (a) after, and (b) before heat treatment at $800{ }^{\circ} \mathrm{C}$

Figure 6-5 TGA plots; (a) $\alpha-\mathrm{Fe}_{2} \mathrm{O}_{3}$, (b) $\alpha-\mathrm{Fe}_{2} \mathrm{O}_{3} / \mathrm{rGO}$ (l) containing $2.9 \mathrm{wt} \%$ of $\mathrm{rGO}$, (c) $\alpha-\mathrm{Fe}_{2} \mathrm{O}_{3} / \mathrm{rGO}$ containing $3.8 \mathrm{wt} \%$ of $\mathrm{rGO}$, and (d) $\alpha-$ $\mathrm{Fe}_{2} \mathrm{O}_{3} / \mathrm{rGO}(\mathrm{h})$ containing $6.1 \mathrm{wt} \%$ of $\mathrm{rGO}$

Figure 6-6 SEM image of the $\alpha-\mathrm{Fe}_{2} \mathrm{O}_{3} / \mathrm{rGO}$ before heat treatment

Figure 6-7 HRTEM image of one of $\alpha-\mathrm{Fe}_{2} \mathrm{O}_{3}$ nanoparticles on the $\mathrm{rGO}$ sheet after heat treatment at $800{ }^{\circ} \mathrm{C}$; The lattice spacing is $3.74 \AA$, which corresponded to the (012) plane of hematite (JCPDS: 330664)

Figure 6-8 XRD patterns of the $\alpha-\mathrm{Fe}_{2} \mathrm{O}_{3}$ and $\alpha-\mathrm{Fe}_{2} \mathrm{O}_{3} / \mathrm{rGO}$

Figure 6-9 UV-visible absorption spectra of $\alpha$-Fe2O3 and $\alpha-\mathrm{Fe}_{2} \mathrm{O}_{3} / \mathrm{rGO}$

Figure 6-10 (a) FTIR spectra and (b) XPS spectra of the $\alpha-\mathrm{Fe}_{2} \mathrm{O}_{3} / \mathrm{rGO}$ before and after heating in $\mathrm{N}_{2}$ at $800^{\circ} \mathrm{C}$

Figure 6-11 XPS spectra of the $\mathrm{Fe} 2 \mathrm{p}$ peaks of (a) $\alpha-\mathrm{Fe}_{2} \mathrm{O}_{3}$ and (b) $\alpha$ $\mathrm{Fe}_{2} \mathrm{O}_{3} / \mathrm{rGO}$ after heating at $800{ }^{\circ} \mathrm{C}$ for activation

Figure 6-12 Normalized $\mathrm{O}_{2}$ evolution plots of the $\alpha-\mathrm{Fe}_{2} \mathrm{O}_{3}$ and $\alpha-\mathrm{Fe}_{2} \mathrm{O}_{3} / \mathrm{rGO}$

Figure 6-13 Photocatalytic stability measurement (a) $\alpha-\mathrm{Fe}_{2} \mathrm{O}_{3} / \mathrm{rGO}$ containing $3.8 \mathrm{wt} \%$ of $\mathrm{rGO}$, (b) $\alpha-\mathrm{Fe}_{2} \mathrm{O}_{3} / \mathrm{rGO}$ (1) containing $2.9 \mathrm{wt} \%$ of $\mathrm{rGO}$, (c) $\alpha-\mathrm{Fe}_{2} \mathrm{O}_{3} / \mathrm{rGO}(\mathrm{h})$ containing $6.1 \mathrm{wt} \%$ of $\mathrm{rGO}$, (d) $\alpha-\mathrm{Fe}_{2} \mathrm{O}_{3}$, and 
(e) physical mixture of $\alpha-\mathrm{Fe}_{2} \mathrm{O}_{3}+\mathrm{rGO}$ containing $3.8 \mathrm{wt} \%$ of $\mathrm{rGO}$

Figure 6-14 Photocurrent densities (J) as a function of the applied voltage (V vs RHE) of the $\alpha-\mathrm{Fe}_{2} \mathrm{O}_{3}$ and $\alpha-\mathrm{Fe}_{2} \mathrm{O}_{3} / \mathrm{rGO}$ with and without irradiation under AM $1.5 \mathrm{G}$ simulated solar light

Figure 6-15 (a) Photocurrent decay curves of the $\alpha-\mathrm{Fe}_{2} \mathrm{O}_{3}$ and $\alpha-\mathrm{Fe}_{2} \mathrm{O}_{3} / \mathrm{rGO}$ at the bias of $1.23 \mathrm{~V}$ vs RHE; (b) Normalized plots of the photocurrent-time dependence for the $\alpha-\mathrm{Fe}_{2} \mathrm{O}_{3}$ and $\alpha-\mathrm{Fe}_{2} \mathrm{O}_{3} / \mathrm{rGO}$

Figure 6-16 Schematic of charge transfer in the $\alpha-\mathrm{Fe}_{2} \mathrm{O}_{3} / \mathrm{rGO}$ composite

Figure 7-1 Characterization of N-LTO plasmonic and heterostructured samples. The TEM (a) and HR-TEM (b) confirmed the presence of Pt and Au nanoparticles on the NLTO/rGO heterostructure, as represented in (c). (d) The band gap of the LTO increased to 550 $\mathrm{nm}$ after doping, with the LSPR of the Au nanoparticles being centered at $550 \mathrm{~nm}$. The addition of rGO led to an increase in absorption across the spectrum.

Figure 7-2 XPS of the (a) Pt, (b) Au, and (c) N peaks for Au@Pt-NLTO/rGO (curve 1), Au@Pt-NLTO (curve 2), Pt-NLTO (curve 3), and $\mathrm{Au} @ \mathrm{Pt}-\mathrm{LTO} / \mathrm{rGO}$ (curve 4).

Figure 7-3 XRD and peak classification for Au@Pt-NLTO/rGO (curve 1), Au@Pt-NLTO (curve 2), Pt-NLTO (curve 3), and Au@Pt$\mathrm{LTO} / \mathrm{rGO}$ (curve 4).

Figure 7-4 Hydrogen generation rate and photocurrent. (a) The interaction of plasmonics and the doped Pt-NLTO with and without heterostructuring with $\mathrm{rGO}$ is seen to outperform the undoped $\mathrm{Au} @ \mathrm{Pt}-\mathrm{LTO} / \mathrm{rGO}$. (b) Similarly, the largest photocurrent is seen for the Au@Pt-NLTO/rGO sample, with the Au@Pt-NLTO and Pt-NLTO both outperforming the doped Au@Pt-LTO/rGO samples. The hydrogen generation and photocurrent show the strong enhancement of combined doping and plasmonics.

Figure 7-5 Photoelectrochemical performance of the four sample: IPCE 112 curves.

Figure 7-6 Hydrogen generation rate over three cycles for Au@Pt- 113 NLTO/rGO, Au@Pt-NLTO, Pt-NLTO, and Au@Pt-LTO/rGO.

Figure 8-1 SEM images obtained from (a) the rGO sheets, (b) the p-MoS $/ \mathrm{n}$ rGO; (c) TEM image of the p-MoS $/ 2$-rGO, and (d) HRTEM image of the $\mathrm{p}-\mathrm{MoS}_{2} / \mathrm{n}$-rGO from the area marked in red square xii 
in $(c)$

Figure 8-2 TEM image of the $\mathrm{MoS}_{2} / \mathrm{rGO}$ composite in which rGO was 128 undoped

Figure 8-3 SEM images of solitary $\mathrm{MoS}_{2}$

Figure 8-4 XRD pattern of the $\mathrm{p}-\mathrm{MoS}_{2} / \mathrm{n}-\mathrm{rGO}$ junctioned photocatalyst

129

Figure 8-5 XPS spectrum of the p-MoS $2 / n-r G O \quad 130$

Figure 8-6 XPS spectra of N1s (a), C1s (b), S2p (c) and Mo 3d (d). The XPS spectra of the $\mathrm{C} 1 \mathrm{~s}$ in (b) was deconvoluted into the sp2 carbon (284.6 eV), C-O-C (285.6 eV), $\mathrm{C}-\mathrm{OH}$ or $\alpha-\mathrm{C}$ in $-\mathrm{C}-\mathrm{COOH}$ $(286.7 \mathrm{eV}), \mathrm{C}=\mathrm{O}(288.0 \mathrm{eV})$ and $\mathrm{COOH}(289.1 \mathrm{eV})$, respectively [17]

Figure 8-7 UV-Visible absorption spectra of the solitary $\mathrm{MoS}_{2}$, the 132 $\mathrm{MoS}_{2} / \mathrm{rGO}$ and the $\mathrm{p}-\mathrm{MoS}_{2} / \mathrm{n}-\mathrm{rGO}$

Figure 8-8 Hydrogen generated by the $\mathrm{MoS}_{2}$, the $\mathrm{MoS}_{2} / \mathrm{rGO}$ and the p- 133 $\mathrm{MoS}_{2} / \mathrm{n}-\mathrm{rGO}$ photocatalysts

Figure 8-9 Mott-Schottky plots of $\mathrm{MoS}_{2} / \mathrm{rGO}$ (a) and $\mathrm{MoS}_{2}$ (b) in the dark (d) and upon light (1) illumination

Figure 8-10 (a) Mott-Schottky plots of the p-MoS $2 / n-r G O$ in dark (d) and under light (l) irradiation; (b) J-V curves of the various catalysts and the bare rGO in dark (d) and under light (l) irradiation; (c) On-off J-t curves of the $\mathrm{MoS}_{2} / \mathrm{rGO}$ and the $\mathrm{p}-\mathrm{MoS}_{2} / \mathrm{n}$-rGO under light (On) in dark (Off) at $-0.1 \mathrm{~V}$ vs. RHE; (d) normalized plots of the photocurrent-time dependence for the $\mathrm{MoS}_{2} / \mathrm{rGO}$ and the $\mathrm{p}$ $\mathrm{MoS}_{2} / \mathrm{n}-\mathrm{rGO}$

Figure 8-11 IPCE of the $\mathrm{MoS}_{2}$, the $\mathrm{MoS}_{2} / \mathrm{rGO}$ and the p-MoS $/ \mathrm{n}-\mathrm{rGO}$ at a 138 bias of $-0.1 \mathrm{~V}$ vs. RHE

Figure 8-12 On-off J-t curve of $\mathrm{MoS}_{2}$ under a bias of $-0.1 \mathrm{~V}$ vs. RHE 


\section{Chapter 1 Introduction}

\subsection{Background}

The demand for energy has increased hand in hand with the increase of the world population and development of human society. It has been reported by the U.S. Energy Information Administration that the total world consumption of marketed energy will increase from 505 quadrillion British thermal units (Btu) in 2008 to 770 quadrillion Btu by 2035 [1]. In the past decade, the major energy source has been fossil fuels, in particular oil [2]. The replacement of fossil fuels is a critical challenge facing society today. First, the waste gases produced from fossil fuel combustion is the leading reason for air pollution. Second, the reserve of fossil fuels will be diminished in the next decade. Third, fuel prices continue to climb as it becomes harder to extract the remaining oil reserves, while consumption continues to grow. These issues have led to research into alternative, next generation energy resources. While, nuclear power has several advantages, safety is still a concern, especially in the aftermath of the 2011 Fukushima Daiichi nuclear disaster $[3,4]$. The most promising alternative energy resource would be renewable, green, and safe.

Fortunately, renewable energy resources are readily being deployed to meet these requirements. For example, by 2010 the renewable energy that is consumed worldwide has already increased to $16.7 \%$, for which biomass accounted $70 \%$ [5]. Besides bio mass conversion, hydropower, wind power, and ethanol are three most commonly used energy sources, accounting for 3.34, 0.51 and $0.50 \%$ of total energy consumption [5]. The most abundant and clean energy source, solar energy, remains to top this list because of low 
conversion efficiencies, with solar energy, including solar hot water, solar photovoltaic power (solar PV power), and concentrated solar power (solar CSP) accounting for less than $2 \%$ of renewable energy produced [5].

\subsection{Motivation}

If solar energy is to replace fossil fuels as a primary or even supplementary energy source extensive improvement is needed. Among solar energy methods, solar to hydrogen conversion is particularly appealing because it directly produces a fuel which can be stored and transported. Even more, the hydrogen produced can be directly used in many currently energy intensive chemical manufacturing processes, further increasing its appeal. It has been reported that the research and development of hydrogen fuel in the United States will cost more than one billion dollars of federal money [6]. $\mathrm{H}_{2}$ research is intensively studied by two national laboratories (National Renewable Energy Laboratory and Sandia National Laboratories) of the Department of Energy (DOE). Hydrogen is useful for energy storage and for use in airplanes and small devices as a power cell, but is not yet practical for automobile compared to using a battery [6].

The multitude possibilities of solar energy to $\mathrm{H}_{2}$ conversion have inspired research into many production routes. In particular, during recent decades much attention has been paid to obtain hydrogen fuel by splitting water with photocatalysts [7-9]. Similar to photosynthesis, photocatalysis is the process of converting solar energy to chemical energy. In this process, solar energy is absorbed by the semiconductor photocatalyst, creating electron and hole pairs, which then reduce or oxidize the water molecules to $\mathrm{H}_{2}$ or to $\mathrm{O}_{2}$ respectively. In order to effectively generate $\mathrm{H}_{2}$ by photocatalytic water splitting, 
the semiconductor photocatalyst must have a sufficiently small band gap to absorb the solar spectrum, while still maintaining the reduction/oxidation levels with water. Briefly, the conduction band should be lower than $0 \mathrm{~V}$ vs $\mathrm{RHE}(\mathrm{pH}=7)$, and valence band should be higher than $1.23 \mathrm{~V}$ vs. $\mathrm{RHE}(\mathrm{pH}=7)$ [10-13]. This creates a balance between solar absorption and water splitting rate which leads to an ideal band gap of around $2 \mathrm{eV}$.

Even if the semiconductor absorbs solar light efficiently and matches the redox potentials of water, it is not guaranteed to be a good photocatalyst. The photoexcited carriers must also be able to reach the surface active photocatalytic sites, requiring long charge recombination lifetimes and large charge migration distances. In this dissertatioin, it will be discussed how nanomaterials can provide a solution to all three problems of poor charge separation, charge recombination, and charge migration facing semiconductor photocatalysts for solar to hydrogen conversion. It has been known that by shrinking photocatalysts to the nanoscale, the surface area can be increased while decreasing the distance charges must migrate to reach surface active sites and drive water splitting. However, with the decrease in size come the additional challenge of poor light absorption and an increase in trap states which decreases carrier lifetime and mobility, effectively reversing the advantage of a nanoscale material [13-17]. Many materials have been investigated for nanoscale photocatalysis to address these problems, but a single material solution has yet to be found. Large band gap metal oxides have good recombination and migration characteristics, but only absorb the $\sim 5 \%$ of the solar spectrum in the UV. Small band gap materials increase solar spectrum utilization, but are plagued by short migration and recombination lifetimes. Clearly, if nanoscale 
photocatalysts are going to be efficient at solar to hydrogen conversion, a materials engineering approach must be employed instead of relying on a single semiconductor.

Therefore, in this dissertation we provide a series of experiments guiding the engineering of single semiconductor photocatalyst materials to address the necessary improvements to charge separation, charge recombination, and charge migration to create a highly efficient solar to hydrogen conversion efficiency. The problem is approached for both large and small band gap materials, providing the necessary steps to improve either the spectral utilization or charge migration/recombination, respectively, in each type of material. This approach allows maximum flexibility in design of a semiconductor photocatalyst by not placing constraints on material selection.

First, in Chapters 3-5, the enhancement of large band gap oxide photocatalysts is investigated by doping. It is shown that dopants must be selected to extend the conduction or valence band edge if a full spectrum enhancement is to be achieved. If the dopant does not form a continuum, mid-gap states are introduced to reduce the charge separation and migration advantages of large band gap materials. This is shown for both $\mathrm{TiO}_{2}$ with the introduction of oxygen vacancies (Chapter 3) and nitrogen doping in $\mathrm{La}_{2} \mathrm{Ti}_{2} \mathrm{O}_{7}$ (LTO) nanosheets (Chapter 4 and 5) [21]. Secondly, in Chapters 6-8, heterostructuring with metal and graphene oxide materials is used to increase charge separation and migration in small band gap semiconductors [18-20]. In Chapter 6 reduced graphene oxide (rGO) is used to quickly extract charge in hematite, a promising material with a $\sim 2 \mathrm{eV}$ band gap but poor carrier lifetimes. Next, in Chapter 7 these charge extraction techniques are combined with the doping of a large band gap semiconductor as optimized in Chapter 3-5 to create an even higher efficiency photocatalyst using $\mathrm{Au}$ 
nanoparticles, LTO, and rGO. Additionally, in Chapter 8, nanoscale p-n junctions at the semiconductor/graphene oxide interface allow highly efficient charge extraction for hydrogen generation in $p-\mathrm{MoS}_{2} / n$-rGO, similar to the p-n junctions used in solar cells for photoelectric generation.

\subsection{Significance}

The results in this dissertation will advance the design of nanoscale photocatalysts for solar to hydrogen conversion. Strategies for the enhancement in solar coverage for large band gap semiconductors and charge extraction enhancements for small band gap semiconductors have been investigated both separately and synergistically. The research into the oxygen vacancy effects in anatase $\mathrm{TiO}_{2}$ has discovered new knowledge as to why

the donor level of $\mathrm{Ti}^{3+}$ increases spectral coverage but often decreases the overall photocatalytic efficiency. The band gap narrowing studies of nitrogen doped LTO nanosheets furthered these new findings, demonstrating that when a continuum of states was formed doping does not have the usual undesirable side effect of decreasing charge lifetime through mid gap states. The heterostructuring of hematite with rGO provided an effective way to overcome the small charge recombination lifetime, and the addition of nanoscale p-n junctions to $\mathrm{MoS}_{2} / \mathrm{rGO}$ allowed photocatalytic activity when $\mathrm{MoS}_{2}$ is not active alone. Further, the heterostructuring of Au/Nitrogen doped LTO/rGO led to increases in spectral coverage and charge extraction through the synergistic interaction of doping, plasmonics, and rGO. Therefore, the presented studies have both explored the fundamental mechanisms of photocatalysis while providing practical guidelines to 
improving performance in real devices and will have a large impact on furthering solar to hydrogen conversion to a stage where it can replace fossil fuel usage in the future.

\section{Reference}

[1] http://www.eia.gov/forecasts/ieo/index.cfm

[2] Schloegl, R. Nat. Mater., 2008, 7, 772-774.

[3] http://en.wikipedia.org/wiki/Nuclear_power

[4] http://www.scientificamerican.com/article.cfm?id=iaea-head-sees-wide-support

[5] http://en.wikipedia.org/wiki/File:Total_World_Energy_Consumption_2010.png

[6] http://en.wikipedia.org/wiki/Sustainable_energy

[7] Fujishima. A, Honda. K, Nature, 1972, 238, 37-38.

[8] Kudo. A, Miseki. Y, Chem. Soc. Rev., 2009, 38, 253-278.

[9] Abe. R, Higashi. M, Sayama. K, Abe. Y, Sugihara. H, J. Phys. Chem. B., 2006, 110, $2219-2226$.

[10] Li. Y, Wang. H, Xie. L, Y. Liang, G. Hong, H. Dai, J. Am. Chem. Soc. 2011, 133, 7296-7299.

[11] Zong. X, Yan. H, Wu. G, Ma. G, Wen. F, Wang. L, Li. C, J. Am. Chem. Soc., 2008, $130,7176-7177$.

[12] Varghese. O. K, Gong. D. W, Paulose. M, Ong. K. G, Ickey. E. C, Grimes. C. A, Adv. Mater., 2003, 15, 624-627.

[13] Wu. N. Q, Zhao. M. H, Zheng. J, Jiang. J. G, Myers, B; Li, S. X.; Chyu, M.; Mao, S. M. Nanotechnology, 2005, 16, 2878-2881. 
[14] Jeong H., Kim T., Kim D., Kim K., Int. J. Hydrogen Energ., 2006, 31, 1142-1146.

[15] Takata T., Furumi Y., Shinohara K., Tanaka A., Hara M., Kondo J. N., Domen K., Chem. Mater. 1997, 9, 1063-1064.

[16] Kim H. G., Hwang D. W., Kim J., Kim Y. J., Lee J. S., Chem. Commun. 1999, 12, 1077-1078.

[17] Tilley, S. D. Cornuz, M. Sivula, K. Gratzel, M. Angew. Chem. Int. Ed., 2010, 49, 6405-6408.

[18] Sivula K., Zboril R., Formal F., Robert R., Weidenkaff A., Tucek J., J. Frydrych, M. Gratzel, J. Am. Chem. Soc. 2010, 132, 7436-7444.

[19] Li L., Yu Y., Meng F., Tan Y., R. J. Hamers, Jin S., Nano Lett, 2011, 12, 4568-4572.

[20] Wang G., Ling Y., Wheeler D. A., George K. E. N., Horsley K., Heske C., Zhang, J. Z., Li Y., Nano Lett. 2011, 11, 3503-3509.

[21] Wang J., Tafen, D. N. Lewis J. P., Hong Z., Manivannan A., Zhi M., Li M., Wu N. Q., J. Am. Chem. Soc., 2009, 13, 12290-12227. 


\section{Chapter 2 Literature Review}

\subsection{Introduction of photocatalytic water splitting}

Photocatalytic water splitting is the process of the conversion of photon energy into $\mathrm{H}_{2}$ as fuel. The ideal case therefore involves using natural solar light to generate $\mathrm{H}_{2}$. Briefly, when dispersed in water solution, the photocatalyst absorbs solar light creating electron and hole pairs, which can then migrate to the surface of the photocatalyst to drive the production of $\mathrm{H}_{2}$ and $\mathrm{O}_{2}$.

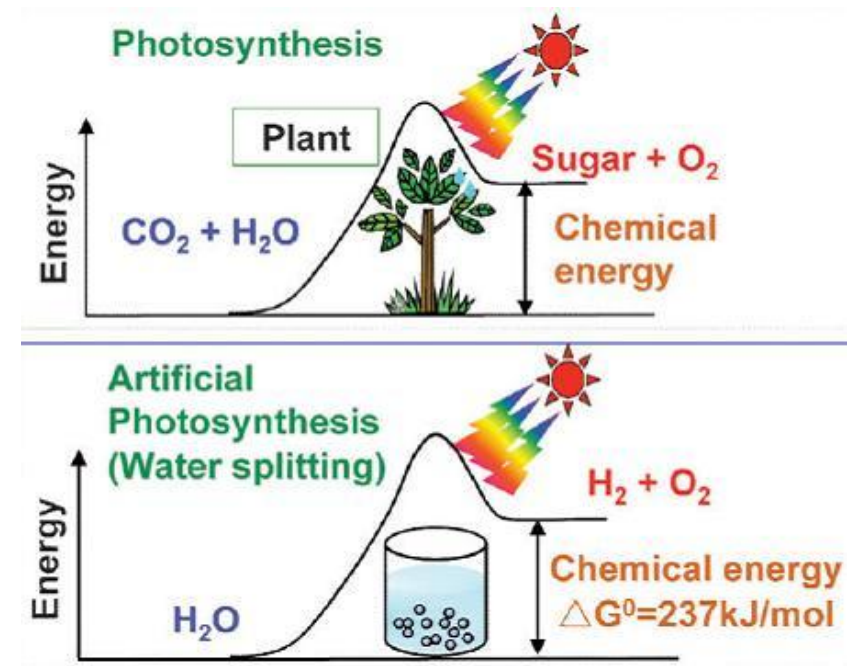

Figure 2-1 Schematic of energy change in photosynthesis and photocatalytic water splitting [1].

Figure 2-1 shows a schematic of photocatalytic water splitting and photosynthesis.

Just as in photosynthesis where the energy from the sun is converted into chemical energy, water splitting converts solar energy into chemical energy as hydrogen and oxygen. Both processes are accompanied by a large, positive change in the Gibbs free 
energy. Because of the similarities, photocatalytic water splitting is considered a type of artificial photosynthesis, and poses many challenging issues in both chemistry and engineering due to the uphill nature of the reaction.

The first experiment for photocatalytic water splitting was conducted by Honda and Fujishima in the early 1970s [3]. It was reported that when $\mathrm{TiO}_{2}$ was irradiated with UV light electron and hole pairs were generated. The photo-generated electrons were able to reduce water to form $\mathrm{H}_{2}$ on a Pt counter electrode and the holes could oxidize water to produce $\mathrm{O}_{2}$ on the $\mathrm{TiO}_{2}$ electrode, but only if some external bias by a power supply or $\mathrm{pH}$ difference between catholyte and anolyte was supplied [3]. Intensive attention has been paid on the study of efficient photocatalysts ever since this discovery. Despite the discovery of a multitude of candidates for photocatalytic water splitting, efficient single materials for water splitting under visible light irradiation remain elusive.

\subsubsection{Processes of photocatalytic water splitting}

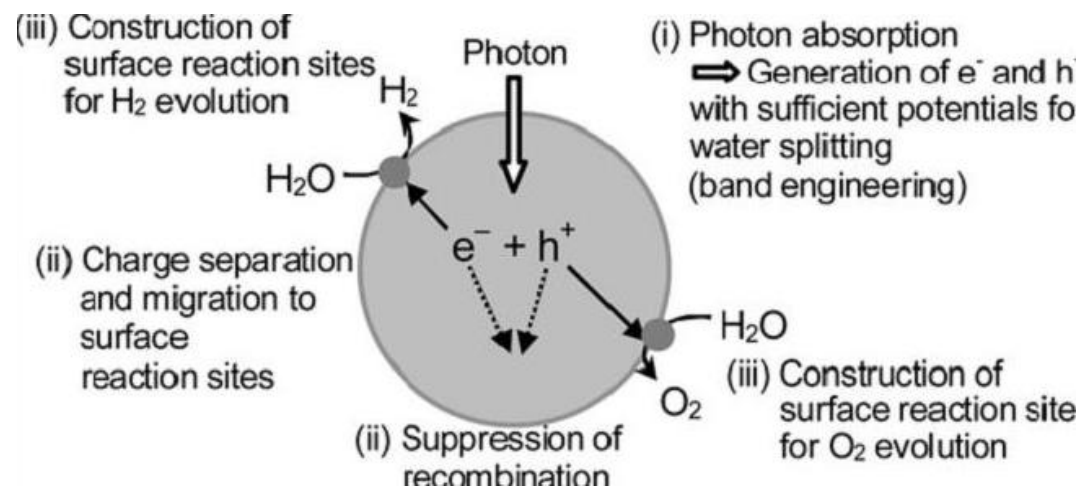

Figure 2-2 Schematic of different processes of photocatalytic water splitting [1]. 
The process of photocatalytic water splitting can be divided into three main steps, which are shown in Figure 2-2 [1]. First, the photocatalyst can absorb photons only if the energy of the incident photons matches the band gap, which is the minimum energy for a photon to excite electrons to the conduction band from valence band. By this process electron and hole pairs are created in the semiconductor photocatalyst. Second, the photoexcited electron hole pairs can either recombine or migrate to different surface reaction sites and drive a chemical reaction. If the excited electrons and holes recombine quickly, the energy will be transferred to heat or re-emitted as photons. If this occurs, the absorbed energy is lost and no product is made, decreasing the efficiency of the energy conversion process.

The suppression of charge recombination is therefore a critical consideration in the design of photocatalysts for water splitting. If the excited electrons and holes do not recombine and can migrate to the surface of the photocatalyst, they can be used for the photocatalytic water splitting reaction. It should be highlighted that crystal structure, crystallinity and particle size all strongly affect this step. Typically, the higher crystalline quality, the higher the activity towards photocatalytic water splitting because less defect sites, such as dislocation and grain boundaries, exist to promote the recombination of electron hole pairs [2]. The effect of particle size of the photocatalyst on the photocatalytic activity is more complicated. On one hand, if the particle size becomes small, the distance for the photo-generated electrons and holes to transfer to reaction sites on the photocatalyst surface becomes short, resulting in a decrease in the overall recombination. On the other hand, the smaller the particle size, the more grain boundaries exist in the photocatalyst, and thus the electron-hole pairs are more likely to recombine. 


\subsubsection{Fundamental theory of photocatalytic water splitting}

As mentioned above, water splitting is an uphill reaction. In equation 2-1, the standard Gibbs free energy change is $237 \mathrm{~kJ} / \mathrm{mol}$, which equates to $1.23 \mathrm{eV}$.

$$
\mathrm{H}_{2} \mathrm{O} \rightarrow \frac{1}{2} \mathrm{O}_{2}+\mathrm{H}_{2} ; \quad \Delta G=+237 \mathrm{~kJ} / \mathrm{mol}
$$

Thus, the minimum band gap energy needed for photocatalytic water splitting reaction is $1.23 \mathrm{eV}$. However, further thermodynamic concerns raises this value to $\sim 2 \mathrm{eV}$ when realistic material properties and chemical kinetics are taken into account. Theoretically, this minimum band gap corresponds to light with a wavelength of about $\sim 620 \mathrm{~nm}$. The relationship of the band gap and light wavelength is described in equation 2-2 [4].

$$
\operatorname{Bandgap}(\mathrm{eV})=1240 / \lambda(\mathrm{nm})
$$

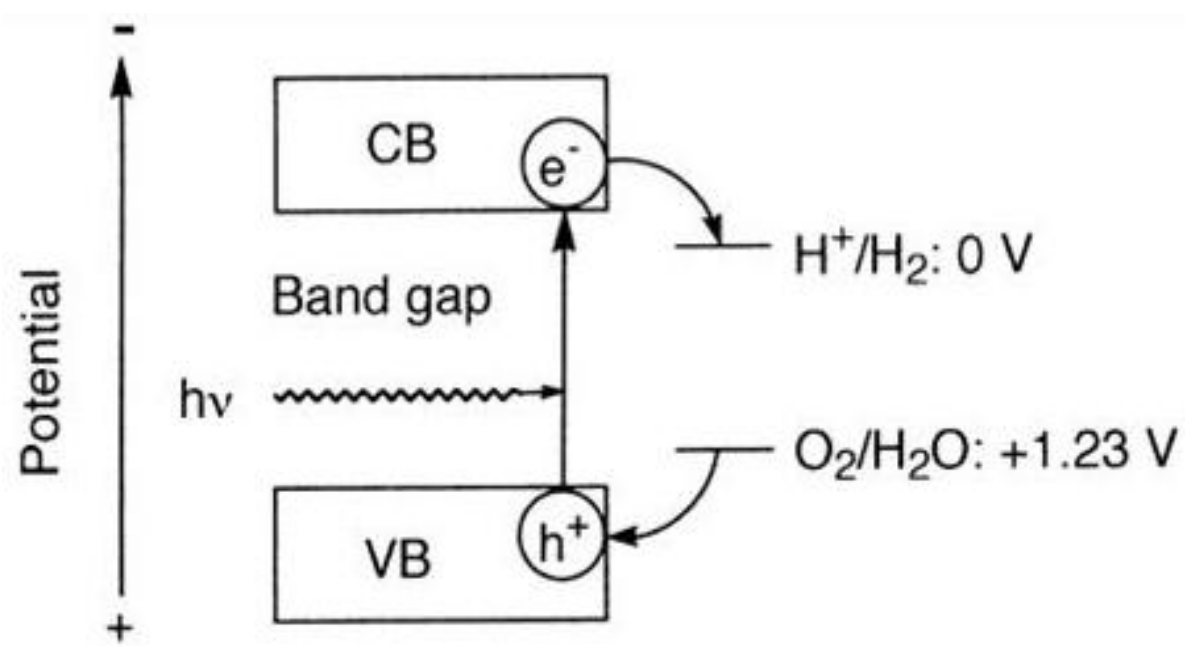

Figure 2-3 Schematic of principle of water splitting using semiconductor photocatalysts [1]. 
A band gap of $\sim 2 \mathrm{eV}$ does not guarantee water splitting is possible. The valance band and conduction band of the photocatalyst must match the redox potentials for the water splitting reaction. Briefly, as shown in Figure 2-3, the conduction band has to be more negative than the potential of $\mathrm{H}^{+} / \mathrm{H}_{2}(0 \mathrm{~V}$ vs. NHE) to ensure reduction, while the top level of the valence band has to be more positive than the potential of $\mathrm{O}_{2} / \mathrm{H}_{2} \mathrm{O}(1.23 \mathrm{~V}$ vs. NHE) for oxidation. If these conditions are met, under illumination the photogenerated electrons and holes in the photocatalyst can reduce and oxidize water molecules to generate $\mathrm{H}_{2}$ and $\mathrm{O}_{2}$, similar to electrolysis.

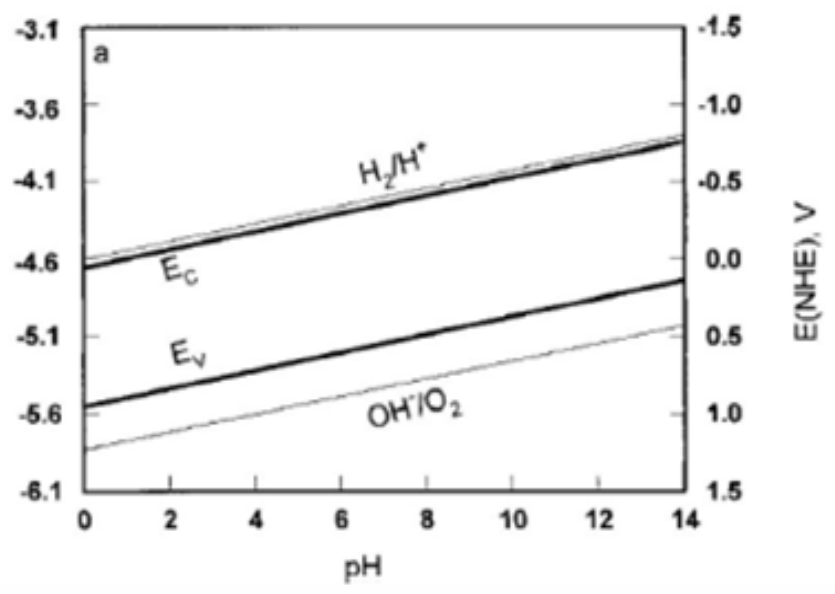

Figure 2-4 $\mathrm{pH}$ dependence of the conduction band and valence band in an aqueous electrolyte solution [4].

The redox potential of water lays out the important material selection criteria that the semiconductor must have both a suitable band gap and positions of the conduction and valence bands. [1]. Even if these conditions are met, the requirements on the band structure is only a thermodynamic requirement, and does not take into account the other important factors of charge separation, mobility, overpotential, and lifetime of photogenerated electrons and holes. All of these factors are important in determining the 
efficiency of the photocatalytic water splitting reactions. For example, one important consideration that must be taken into account is that the band edges (Figure 2-4) of the semiconductor vary with the change of $\mathrm{pH}[4]$.

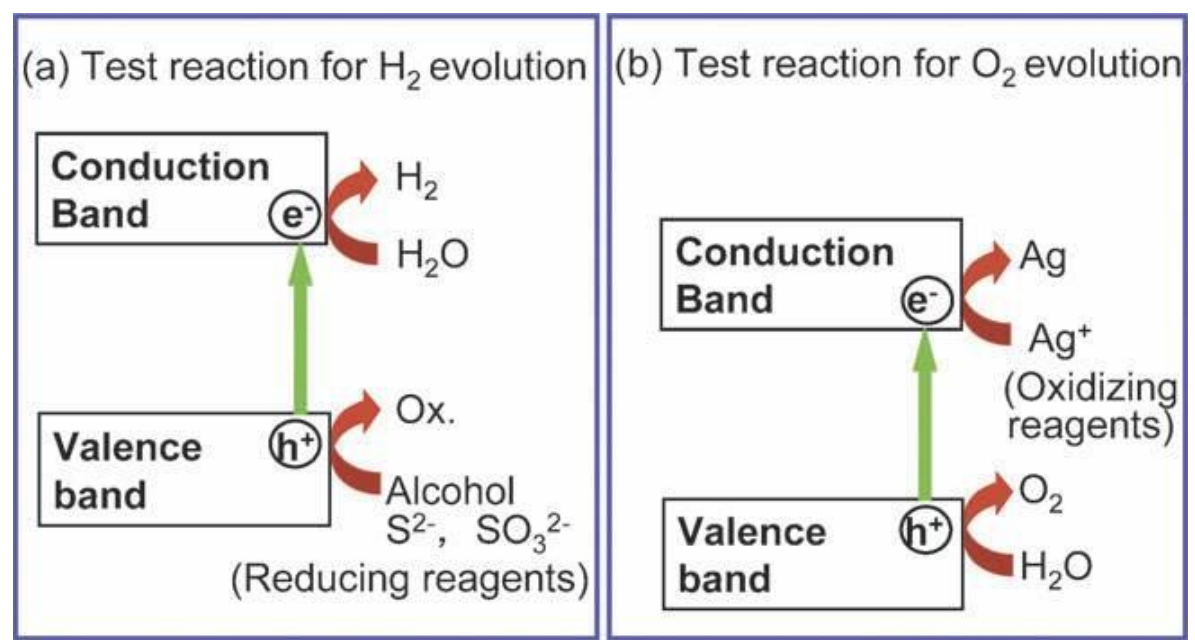

Figure 2-5 Schematic of half reactions of water splitting $-\mathrm{H}_{2}$ or $\mathrm{O}_{2}$ evolution reactions

The long list of requirements makes the complete splitting of water difficult. Instead, the water splitting reaction is usually investigated as two half reactions (water reduction for $\mathrm{H}_{2}$ evolution in Figure 2-5 (a) and water oxidation for $\mathrm{O}_{2}$ evolution in Figure 2-5 (b)). In these cases, sacrificial reagents are often employed to balance the whole photocatalyst system and compensate for the missing half of the water splitting reaction. As shown in Figure 2.5, the commonly used sacrificial reagents for holes scavengers are $\mathrm{S}^{2-}, \mathrm{SO}_{3}{ }^{2-}$, and alcohols. Hole scavengers can give electrons to suppress the extra holes left in the photocatalyst after hydrogen production. If the holes are not compensated in the photocatalyst, photo-corrosion occurs (ex. equation 2-3 and 2-4) and damages the photocatalyst. 


$$
\begin{aligned}
& \mathrm{CdS}+2 h^{+} \rightarrow \mathrm{Cd}^{2+}+S \\
& \mathrm{ZnO}+2 h^{+} \rightarrow \mathrm{Zn}^{2+}+1 / 2 \mathrm{O}_{2}
\end{aligned}
$$

On the contrary, for $\mathrm{O}_{2}$ evolution an electron scavenger such as $\mathrm{AgNO}_{3}$ or $\mathrm{FeCl}_{3}$ is needed as the sacrificial reagent to suppress the extra electrons in the photocatalyst after oxygen production.

\subsubsection{Evaluation of photocatalytic water splitting}

Many methods exist for the evaluation of photocatalytic water splitting. The typical indicators are listed below:

(1) Normalized evaluation rates of $\mathrm{H}_{2}$ or $\mathrm{O}_{2}$ production

The activity of photocatalytic water splitting reaction is calculated by the amount of $\mathrm{H}_{2}$ or $\mathrm{O}_{2}$ evolved under light irradiation in a certain time. Many factors related to instrument design, such as lamps and irradiation configurations, will affect the measured production rate. Therefore, a normalized rate of $\mathrm{H}_{2}$ or $\mathrm{O}_{2}$ evolution is used employed to allow comparison of different photocatalysts and experimental conditions. Generally, the rate unit used for gas evolution is $\mu \mathrm{mol} \cdot \mathrm{h}^{-1} \cdot \mathrm{g}^{-1}$. As long as the irradiation light source and solution systems employed in the photocatalysis experiments are described, different photocatalyst in different experimental conditions should be comparable.

(2) Turn over number (TON)

In the former section, the normalized gas rate is used to evaluate the gas production rate. However, different catalysts have different densities of active sites on 
which the catalytic reaction occurs. Therefore, it is difficult to evaluate the efficiency of the photocatalytic water splitting reaction. The turn over number (TON) is used to solve this problem. TON is usually defined by the ratio of the number of reacted molecules to that of an active site, as shown in equation 2-5.

$$
\text { TON }=\frac{\text { Number of reacted molecules }}{\text { Number of active sites }}
$$

However, for inorganic photocatalysts, it is difficult to calculate the number of active sites so the number of active sites is usually changed to the number of atoms on the surface of the photocatalyst. Equation 2-5 the becomes

$$
\text { TON }=\frac{\text { Number of reacted molecules }}{\text { Number of atoms at the surface of a photocatalyst }}
$$

\section{(3) Quantum yield}

Another factor for the evaluation of the efficiency of energy usage is the quantum yield. Because of the strong scattering in the dispersed solution system, it is hard to determine the exact amount of photons absorbed by a photocatalyst and the exact ratio of energy absorbed to energy converted can not be determined. Therefore, the apparent quantum yield (AQY) is introduced in equation 2-7.

$$
A Q Y=\frac{\text { Number of reacted electrons }}{\text { Number of incident photons }} \times 100 \%
$$




\subsection{Materials for photocatalysis}

A large number of semiconductor materials have been developed as photocatalysts. Most of these materials are UV-responsive photocatalysts, which can be classified into the following four groups.

The semiconductor materials consisting of $\mathrm{d}^{0}$ metal cations, such as $\mathrm{Ti}^{4+}, \mathrm{Zr}^{4+}$, $\mathrm{Nb}^{5+}$ and $\mathrm{Ta}^{5+}$, are the most intensively studied photocatalyst [5-17]. Solid state reaction (SSR) methods are usually used to prepare these mixed metal oxides. Briefly, starting materials containing a metal oxides and/or alkali and alkaline earth carbonates are homogenously mixed and then calcined at high temperatures in air $[13,15]$. A polymerizable complex method can also be used for preparation of these photocatalysts $[9,11,14,18]$. This synthesis method gives fine, crystalline powders as a product. The surface area is also generally higher than the SSR method, due to the relatively low calcination temperature and short calcination time.

$\mathrm{TiO}_{2}$ is the first reported photocatalyst for water splitting [3]. Recently, a series of titanates with perovskite structure were developed as efficient photocatalysts for photocatalytic water splitting [19-23]. For example, $\mathrm{K}_{2} \mathrm{Ti}_{2} \mathrm{O}_{5}, \mathrm{~K}_{2} \mathrm{Ti}_{4} \mathrm{O}_{9}, \mathrm{La}_{2} \mathrm{Ti}_{2} \mathrm{O}_{7}$ and $\mathrm{Na}_{2} \mathrm{Ti}_{3} \mathrm{O}_{7}$ are active for photocatalytic $\mathrm{H}_{2}$ generation in aqueous solution with the assistance of methanol or ethanol as a hole scavenger [19-21]. In order to increase the activity of photocatalytic water splitting, noble metal particles (Pt, $\mathrm{Ru}$, etc.) are usually deposited on the surface of the photocatalyst [20].

Another type of metal oxide like $\mathrm{TiO}_{2}$ is $\mathrm{ZrO}_{2}$. More than one decade ago it was found that $\mathrm{ZrO}_{2}$ powder could split pure water without any deposition of cocatalysts 
under UV light $[24,25]$. Furthermore, the photocatalytic activity of $\mathrm{ZrO}_{2}$ can be significantly increased by the pressure of the reaction system and $\mathrm{pH}$ value of the solution. Interestingly, unlike other semiconductors, loading noble metal particles decreases the performance of photocatalytic water splitting for $\mathrm{ZrO}_{2}$. Unfortunately, the mechanism of decreased performance for water splitting is still unclear.

$\mathrm{Nb}$ - and Ta- groups of metal oxide semiconductors were also studied intensively as an effective photocatalyst. The related strontium niobates $\mathrm{SrNb}_{2} \mathrm{O}_{6}, \mathrm{Sr}_{5} \mathrm{Nb}_{4} \mathrm{O}_{15}$, and $\mathrm{Sr}_{2} \mathrm{Nb}_{2} \mathrm{O}_{7}$ showed efficient photocatalytic activity for both hydrogen and oxygen generation from pure water [26-28]. $\mathrm{Nb}_{2} \mathrm{O}_{5}$ is not active for pure water splitting even under UV irradiation, however, the photocatalytic $\mathrm{H}_{2}$ generation performance of $\mathrm{Nb}_{2} \mathrm{O}_{5}$ exists once modified with Pt and put in solution with methanol [29, 30]. Ta-based oxides only have photocatalytic $\mathrm{H}_{2}$ generation performance under UV irradiation, and a large band gap of $\sim 4.0 \mathrm{eV}$ [29]. The photocatalytic $\mathrm{H}_{2}$ generation rate of $\mathrm{Ta}_{2} \mathrm{O}_{5}$ can be increased significantly by modification with $\mathrm{NiO}$ and $\mathrm{RuO}_{2}$ as the cocatalysts on the surface [29]. It was reported that alkali tantalates $\mathrm{XTaO}_{3}(\mathrm{X}: \mathrm{Li}, \mathrm{Na}$ or $\mathrm{K})$ showed impressive activities for photocatalytic water splitting for both $\mathrm{H}_{2}$ and $\mathrm{O}_{2}$ generation under UV light [31].

The typical $\mathrm{d}^{10}$ group metal oxide photocatalysts include metal oxides containing $\mathrm{In}^{3+}, \mathrm{Ga}^{3+}, \mathrm{Ge}^{4+}, \mathrm{Sn}^{4+}$ and $\mathrm{Sb}^{5+}$ cations [32-35]. They exhibit excellent photochemical water splitting activities under UV light irradiation. Among of them, Ni-loaded $\mathrm{Ga}_{2} \mathrm{O}_{3}$ was a promising photocatalyst for overall water splitting [32]. Especially, $\mathrm{Zn}$ ion doping notably increased the photocatalytic activity of $\mathrm{Ga}_{2} \mathrm{O}_{3}$ for water splitting, and the apparent quantum yield reached up to $20 \%$. 


\subsection{Approaches for improvement of photocatalytic activity}

\subsubsection{Metal/Nonmetal ion doping and codoping}

One major avenue of enhancement is increasing the spectral coverage of the semiconductor by doping. Ion doping techniques have been studied by many researchers, because of the ease of fabrication and large potential improvement of the photocatalytic water splitting performance. The three typical methods for doping are metal doping, nonmetal doping and metal/nonmental codoping.

More than 30 years ago, researchers found that the photocatalytic water splitting

performance of $\mathrm{TiO}_{2}$ can be improved by doping with $\mathrm{Cr}^{5+}$ [36]. Many metal cations have since been doped into $\mathrm{TiO}_{2}$ to improve its performance for photocatalytic water splitting such as, Cr, Fe, Mn, Sn, V, Mo, and Ni. [36-46]. The electronic structures of $\mathrm{TiO}_{2}$ doped with the $3 \mathrm{~d}$ transition metals have been studied in depth by theoretical calculations. It has been reported that doping $3 \mathrm{~d}$ metal ions into $\mathrm{TiO}_{2}$ mainly creates donor levels in the band gap or close to the conduction band [45].

Various nonmetal anions (such as $\mathrm{S}, \mathrm{C}, \mathrm{N}$, etc.) have also been doped into $\mathrm{TiO}_{2}$. The light absorption properties of $\mathrm{TiO}_{2}$ were significantly changed by inclusion of these nonmetal ion dopants. For example, $\mathrm{TiO}_{2}$ showed a clear red-shift of the band edge to longer wavelengths by doping with $\mathrm{C}, \mathrm{N}$, or S [47-49]. The extended light absorption range increases the efficiency of solar energy conversion. The donor levels in these materials were proven by X-ray photoelectron spectroscopy (XPS) to be close to valence band of $\mathrm{TiO}_{2}$, opposite from the donor levels introduced by metal ion doping [47]. 
Both metal doping and nonmetal doping are considered to be single ion doping techniques. While doping extends the band edge, vacancies are also introduced into the $\mathrm{TiO}_{2}$ lattice during the doping process. These vacancies increase charge recombination and trapping, decreasing the overall enhancement in solar energy conversion. In order to maintain the charge balance and decrease the introduction of vacancies, researchers tried to dope both cations and anions together into $\mathrm{TiO}_{2}$. Many ion couples were used in the codoping systems, for example, $\mathrm{Fe}+\mathrm{N}$ or $\mathrm{C} ; \mathrm{Ni}+\mathrm{B}$ or $\mathrm{N}$; $\mathrm{Ce}+\mathrm{C}, \mathrm{I}, \mathrm{N}$, or B; $\mathrm{La}+\mathrm{N}, \mathrm{I}$, or $\mathrm{S} ; \mathrm{Bi}+\mathrm{S}, \mathrm{C}$, or $\mathrm{N}$ [50-62]. The performance of codoped $\mathrm{TiO}_{2}$ was better than either pristine $\mathrm{TiO}_{2}$ or $\mathrm{TiO}_{2}$ with single ion dopants.

\subsubsection{Cocatalyst loading}

Whereas doping extends the spectral coverage and increases initial charge creation, loading cocatalysts onto the surface of the photocatalyst extracts the photogenerated charge carriers, suppressing charge recombination and increasing the apparent quantum yield for absorbed energy. In the following part, three typical cocatalysts used for surface loading will be discussed.

(1) Noble Metal Cocatalyst

When noble metals are deposited on a photocatalyst the photo-generated electrons accumulate on the metal particles, delaying the recombination of the electron-hole pairs, and boosting the hydrogen evolution efficiency [69]. Among all the noble metals, Pt is the most widely used cocatalyst [63-69]. All have been shown to greatly enhance the photocatalytic activity for hydrogen evolution. Up until now, the highest photocatalytic activities for hydrogen production from water using visible-light irradiation are from 
photocatalysts loaded with $\mathrm{Pt}$ as the cocatalyst. Other metals, such as $\mathrm{Au}, \mathrm{Ag}$, and $\mathrm{Ru}$, working as cocatalysts also exhibited efficient photocatalytic activity for hydrogen evolution [70-75].

(2) Metal oxide cocatalysts

The most used metal oxides as cocatalyst were $\mathrm{RuO}_{2}$ and $\mathrm{NiO}$ [76-79]. Actually, $\mathrm{RuO}_{2}$ and $\mathrm{NiO}$ are independent photocatalysts, and when coupled with other semiconductors, they can work together to improve the charge separation. Most of these metal oxides are good candidates for water oxidation to generate $\mathrm{O}_{2}$ under UV light irradiation. The typical example is $\mathrm{IrO}_{2}$, which can used as an efficient water oxidation cocatalyst $[80]$.

(3) Nonmetal-oxide cocatalysts

During recent decade, some nonmetal-oxide cocatalysts have attracted more and more attention. The typical examples are $\mathrm{MoS}_{2}$ and $\mathrm{CdS}$ nanoparticles [81-82]. These cocatalysts can work as a type of sensitizer and give good catalytic sites for $\mathrm{H}_{2}$ generations. It should be clarified that the electrons are not directly transferred from the photocatalysts to $\mathrm{MoS}_{2}$ to reduce water molecules. Instead, the electrons react with water at the active sites on the interface of $\mathrm{MoS}_{2}$ and the semiconductor photocatalyst to generate $\mathrm{H}_{2}$, opposite of the previous cocatalysts [81].

\section{Reference}

[1] Kudo A., Miseki Y., Chem. Soc. Rev. 2009, 38, 253-278.

[2] Kudo A., Catal. Surv. Asia, 2003, 7, 31-38.

[3] Fujishima A., K. Honda, Nature 1972, 238, 37-38. 
[4] Chen X. B.., Shen. S. H., Guo. L. J., Mao. S. S., Chem. Rev. 2010, 110, 6503-6570

[5] Sayama K., Arakawa H., J. Chem. Soc., Faraday Trans., 1997, 93, 1647.

[6] Tabata S., Nishida N., Masaki Y., Tabata K., Catal. Lett., 1995, 34, 245.

[7] Moon S. C., Mametsuka H., Suzuki E., Anpo, M., Chem. Lett., 1998, 27, 117.

[8] Jeong, H., Kim T., Kim D. , Kim K., Int. J. Hydrogen Energy., 2006, 31, 1142.

[9] Ko Y. G., Lee, W. Y, Catal. Lett., 2002, 83, 157.

[10] Kim J., Hwang D. W., Kim H. G., Bae S. W., Lee J. S., Li W. , O S. H. h, Top. Catal., 2005, 35, 295.

[11] Y. Miseki, H. Kato and A. Kudo, Chem. Lett., 2005, 34, 54.

[12] Abe, R., Higashi, M., Zou, Z. G., Sayama, K., Arakawa, H., J. Phys. Chem. B, 2004, $108,811$.

[13] Saito, N., Kadowaki, H., Kobayashi, H., Ikarashi, K., Nishiyama, H., Inoue, Y., Chem. Lett., 2004, 33, 2004.

[14] Kadowaki, H., Saito, N, Nishiyama, H., Kobayashi, H., Shimodaira, Y., Inoue, Y., J. Phys. Chem. C, 2007, 111, 439.

[15] Ikeda, S., Itani, Nango, K. T., Matsumura, M., Catal. Lett., 2004, 98, 229.

[16] Kadowaki, H., Saito, N., Nishiyama, H., Inoue, Y., Chem. Lett., 2007, 36, 440.

[17] Yuan, Y., Zheng, J., Zhang, X., Li, Z., Yu, T., Ye, J., Zou, Z., Solid State Ionics, 2008, 178, 1711.

[18] Kakihana, M., Domen, K., MRS Bull., 2000, 25, 27.

[19] Shibata, M.; Kudo, A.; Tanaka, A.; Domen, K.; Maruya, K.; Ohishi, T. Chem. Lett. 1987, 16, 1017. 
[20] Allen, M. R.; Thibert, A.; Sabio, E. M.; Browning, N. D.; Larsen, D. S.; Osterloh, F. E. Chem. Mater. 2010, 22, 1220.

[21] Sun, W.; Zhang, S.; Wang, C.; Liu, Z.; Mao, Z. Catal. Lett. 2007, 119, 148.

[22] Kim, A.; Hwang, D. W.; Bae, S. W.; Kim, Y. G.; Lee, J. S., Korean J. Chem. Eng. 2001, 18, 941.

[23] Kim, J.; Hwang, D. W.; Kim, H. G.; Bae, S. W.; Ji, S. M.; Lee, J. S. Chem. Commun. 2002, 21, 2488.

[24] Sayama, K.; Arakawa, H. J. Phys. Chem. 1993, 97, 531.

[25] Sayarna, K.; Arakawa, H. J. Photochem. Photobiol., A 1996, 94, 67.

[26] Miseki, Y.; Kato, H.; Kudo, A. Energy EnViron. Sci. 2009, 2, 306.

[27] Kim, H. G.; Hwang, D. W.; Kim, J.; Kim, Y. G.; Lee, J. S. Chem. Commun. 1999, $12,1077$.

[28] Chen, D.; Ye, J. Chem. Mater. 2009, 21, 2327.

[29] Sayama, K.; Arakawa, H.; Domen, K. Catal. Today 1996, 28, 175.

[30] Chen, X.; Yu, T.; Fan, X.; Zhang, H.; Li, Z.; Ye, J.; Zou, Z. Appl. Surf. Sci. 2007, 253,8500 .

[31] Kato, H.; Kudo, A. Chem. Phys. Lett. 1998, 295, 487.

[32] Yanagida, T.; Sakata, Y.; Imamura, H. Chem. Lett. 2004, 33, 726.

[33] Sakata, Y.; Matsuda, Y.; Yanagida, T.; Hirata, K.; Imamura, H.; Teramura, K. Catal. Lett. 2008, 125, 22.

[34] Wang, D.; Zou, Z.; Ye, J. Chem. Phys. Lett. 2004, 384, 139.

[35] Kudo, A.; Mikami, I. J. Chem. Soc., Faraday Trans. 1998, 94, 2929. 
[36] Borgarello, E.; Kiwi, J.; Gratzel, M.; Pelizzetti, E.; Visca, M. J. Am. Chem. Soc. 1982, 104, 2996.

[37] Chen, X.; Mao, S. S. Chem. Rev. 2007, 107, 2891.

[38] Ji, P. F.; Takeuchi, M.; Cuong, T. M.; Zhang, J. L.; Matsuoka, M.; Anpo, M. Res. Chem. Intermed. 2010, 36, 327.

[39] Leung, D. Y. C.; Fu, X. L.; Wang, C. F.; Ni, M.; Leung, M. K. H.; Wang, X. X.; Fu, X. Z. ChemSusChem, 2010, 3, 681.

[40] Liu, J.; Chen, G.; Lia, Z.; Zhang, Z. J. Solid State Chem. 2006, 179, 3704.

[41] Hwang, D. W.; Kim, H. G.; Lee, J. S.; Li, W.; Oh, S. H. J. Phys. Chem. B 2005, 109, 2093.

[42] Tian, B.; Li, C.; Gu, F.; Jiang, H.; Hu, Y.; Zhang, J. Chem. Eng. J. 2009, 151, 220.

[43] Devi, L. G.; Kumar, S. G.; Murthy, B. N.; Kottam, N. Catal. Commun. 2009, 10, 794.

[44] Takaoka, G. H.; Nose, T.; Kawashita, M. Vacuum 2008, 83, 679.

[45] Fan, X.; Chen, X.; Zhu, S.; Li, Z.; Yu, T.; Ye, J.; Zou, Z. J. Mol. Catal. A: Chem. 2008, 284, 155 .

[46] Kim, D. H.; Choi, D. K.; Kim, S. J.; Lee, K. S. Catal. Commun. 2008, 9, 654.

[47] J. Wang, D. N. Tafen, J. P. Lewis, Z. Hong, A. Manivannan, M. Zhi, M. Li, N. Q. Wu, J. Am. Chem. Soc., 2009, 131, 12290-12227.

[48] Chen, X.; Glans, P. A.; Qiu, X.; Dayal, S.; Jennings, W. D.; Smith, K. E.; Burda, C.; Guo, J. J. Electron Spectrosc. Relat. Phenom. 2008, 162, 67.

[49] Chen, X.; Burda, C. J. Am. Chem. Soc. 2008, 130, 5018.

[50] Xu, J.; Ao, Y.; Fu, D. Appl. Surf. Sci. 2009, 256, 884. 
[51] Song, S.; Tu, J.; Xu, L.; Xu, X.; He, Z.; Qiu, J.; Ni, J.; Chen, J. Chemosphere 2008, $73,1401$.

[52] Liu, C.; Tang, X.; Mo, C.; Qiang, Z. J. Solid State Chem. 2008, 181, 913.

[53] Wei, C.; Tang, X.; Liang, J.; Tan, S.; J. Environ. Sci. 2007, 19, 90.

[54] Tryba, B. Int. J. Photoenergy., 2008, 72, 1824.

[55] Shen, X. Z.; Guo, J.; Liu, Z. C.; Xie, S. M. Appl. Surf. Sci. 2008, 254, 4726.

[56] Lv, K.; Zuo, H.; Sun, J.; Deng, K.; Liu, S.; Li, X.; Wang, D. J. Hazard. Mater. 2009, $161,396$.

[57] Wang, Y.; Wang, Y.; Meng, Y.; Ding, H.; Shan, Y.; Zhao, X.; Tang, X. J. Phys. Chem. C 2008, 112, 6620.

[58] Huang, Y.; Ho, W.; Ai, Z.; Song, X.; Zhang, L.; Lee, S. Appl. Catal. B., 2009, 89, 398.

[59] Zhang, X.; Liu, Q. Appl. Surf. Sci. 2008, 254, 4780.

[60] Xia, H.; Zhuang, H.; Xiao, D.; Zhang, T. J. Alloys Compd. 2008, 465, 328.

[61] He, Z.; Xu, X.; Song, S.; Xie, L.; Tu, J.; Chen, J.; Yan, B. J. Phys. Chem. C, 2008, $112,16431$.

[62] Liu, Z.; Zhou, Y.; Li, Z.; Wang, Y.; Ge, C. Rare Met. 2007, 26, 263.

[63] Ikuma, Y.; Bessho, H. Int. J. Hydrogen Energy 2007, 32, 2689.

[64] Sobczynski, A.; Yildiz, A.; Bard, A. J.; Campion, A.; Fox, M. A.; Mallouk, T.; Webber, S. E.; White, J. M. J. Phys. Chem. 1988, 92, 2311.

[65] Jang, J. S.; Joshi, U. A.; Lee, J. S. J. Phys. Chem. C 2007, 111, 13280.

[66] Bessekhouad, Y.; Mohammedi, M.; Trari, M. Sol. Energy Mater. Sol. Cells 2002, 73, 339. 
[67] Bao, N.; Shen, L.; Takata, T.; Lu, D.; Domen, K. Chem. Lett. 2006, 35, 318.

[68] Xiao, W.; Yuan, J.; Zhang, Y.; Shangguan, W. Mater. Chem. Phys. 2007, 105, 6.

[69] Meng, F. K.; Li, J. T.; Hong, Z. L.; Zhi, M. J.; Sakala, A.; Xiang, C. C.; Wu, N. Q.;

Catal. Today., DOI: 10.1016/j.cattod.2012.05.038

[70] Chiarello, G. L.; Selli, E.; Forni, L. Appl. Catal., B 2008, 84, 332.

[71] Sreethawong, T.; Yoshikawa, S. Catal. Commun. 2005, 6, 661.

[72] Navarro, R. M.; del Valle, F.; Fierro, J. L. G. Int. J. Hydrogen Energy 2008, 33, 4265.

[73] Lu, W.; Gao, S.; Wang, J. J. Phys. Chem. C 2008, 112, 16792.

[74] Anandan, S.; Kumar, P. S.; Pugazhenthiran, N.; Madhavan, J.; Maruthamuthu, P. Sol. Energy Mater. Sol. Cells 2008, 92, 929.

[75] Hou, X. G.; Huang, M. D.; Wu, X. L.; Liu, A. D. Chem. Eng. J. 2009, 146, 42.

[76] Arai, N.; Saito, N.; Nishiyama, H.; Domen, K.; Kobayashi, H.; Sato, K.; Inoue, Y. Catal. Today 2007, 129, 407.

[77] Ebina, Y.; Sakai, N.; Sasaki, T. J. Phys. Chem. B 2005, 109, 17212.

[78] Sreethawong, T.; Suzuki, Y.; Yoshikawa, S. Int. J. Hydrogen Energy 2005, 30, 1053.

[79] Lin, H.; Chen, Y.; Chen, Y. Int. J. Hydrogen Energy 2007, 32, 86.

[80] Ma, B.; Yang, J.; Han, H.; Wang, J.; Zhang, X.; Li, C. J. Phys. Chem. C 2010, 114, 12818.

[81] Zong, X.; Yan, H.; Wu, G.; Ma, G.; Wen, F.; Wang, L.; Li, C. J. Am. Chem. Soc. 2008, $130,7176$.

[82] Zong, X.; Wu, G.; Yan, H.; Ma, G.; Shi, J.; Wen, F.; Wang, L.; Li, C. J. Phys. Chem. C 2010, 114, 1963. 


\section{Chapter 3 Effects of Oxygen Vacancy to the Photocatalytic}

\section{Activity of Anatase $\mathrm{TiO}_{2}$}

\subsection{Background introduction}

As mentioned in Chapter 1 , titanium dioxide $\left(\mathrm{TiO}_{2}\right)$ has been widely studied because of its prospective future in solar energy conversion and environmental cleanup [1-7]. While $\mathrm{TiO}_{2}$ has great surface kinetics, charge recombination, and charge migration characteristics, the possibility of high efficiency is sabotaged by the low spectral coverage of its large band gap $(\sim 3.2 \mathrm{eV})$, which allows only UV light (accounting for less $5 \%$ natural solar light) to be utilized [7]. Despite its large band gap, $\mathrm{TiO}_{2}$ remains one of the most successful catalysts to date, so extending the light-absorption regime remains a high priority. The most commonly used technique for improving the light-absorption efficiency of $\mathrm{TiO}_{2}$ is doping metal cations or nonmetal anions into the $\mathrm{TiO}_{2}$ lattice [7-16]. Cationic doping creates $d$ bands deep into the band gap of $\mathrm{TiO}_{2}$, while anion doping creates $p$ bands near valence band of $\mathrm{TiO}_{2}[7,12]$. The sub-bands created by doping allow for electrons to be photoexcited into the conduction or valance band with less energy, decreasing the band gap of the semiconductor, and extending the light-absorption regime from the UV to longer wavelengths (normally 400-600 nm) [7, 17, 18]. Therefore, the efficiency of solar-light-energy utilization in $\mathrm{TiO}_{2}$ photocatalysts should be greatly increased when doped.

Although many studies have been performed for ion-doping methods in $\mathrm{TiO}_{2}$, the conclusions are often contradictory. Although the solar light absorption range is always 
increased, the overall photocatalysis is often worse. Particularly, an increase in visible light photocatalysis often leads to a decrease in UV photocatalysis. When a dopant is introduced into $\mathrm{TiO}_{2}$ oxygen vacancies commonly form [7-14]. In fact, one popular form of doping involves purposely introducing oxygen vacancies to extend the absorption of $\mathrm{TiO}_{2}[8,19]$. It has also been discovered that the oxygen vacancy on rutile $\mathrm{TiO}_{2}$ surface can adsorb gas molecules, which might be helpful for photocatalysis [1,2]. Almost all of the research to date involving oxygen vacancies is on single crystal rutile $\mathrm{TiO}_{2}$, while much less work involves anatase titanium dioxide despite its more favorable photocatalytic activity $[1,19-22]$. Although it would seem oxygen vacancies are central to understanding the effects of doping on photocatalysis, the relationship remains unclear.

To clarify this critical point, we have investigated the effects of oxygen vacancies in anatase $\mathrm{TiO}_{2}$. First, a hydrothermal method was employed to synthesize pristine singlecrystalline anatase nanobelts. Then, reduced anatase nanobelts were obtained in $\mathrm{H}_{2}$ flow at high temperatures to ensure only oxygen was removed without other dopants being added into the $\mathrm{TiO}_{2}$ lattice. Single-crystalline anatase nanobelts were chosen as the photocatalyst due to the following reasons. First, free electron diffusion favors the onedimensional geometric structure of nanobelts over nanoparticles because the long direction acts as a diffusion channel $[23,24]$. Second, the high length-to-diameter ratio of nanobelts is able to enhance visible-light scattering and absorption [25, 26]. Third and most importantly, the synthesized nanobelts are of a high degree of crystallinity and have a low number of grain boundaries, reducing the effects of electron-hole recombination at the grain boundaries, and deconvoluting the effect of oxygen vacancies from other forms of defect states. [27] 


\subsection{Experimental section}

\subsubsection{Synthesis of the pristine and reduced $\mathrm{TiO}_{2}$ nanobelts}

The synthesis procedure of pristine $\mathrm{TiO}_{2}$ nanobelts is described briefly in the following as in our previous works $[7,15,16]$. First, $1.0 \mathrm{~g}$ of anatase $\mathrm{TiO}_{2}$ particles (232033, Sigma-Aldrich) and $70 \mathrm{~mL}$ of $10 \mathrm{M} \mathrm{NaOH}$ aqueous solution were mixed in a 80 $\mathrm{mL}$ Teflon cylinder to form a white suspension. The suspension was stirred rigorously with a glass stick for 2 min and then placed in a Teflon cylinder in an ultrasonic bath for another 30 min to make sure the suspension was mixed homogeneously. Second, the sealed Teflon cylinder was placed in an autoclave and a preheated furnace $\left(200^{\circ} \mathrm{C}\right)$ for 24 h. Hydrothermal synthesis occurred at this temperature. Third, after hydrothermal processing, the autoclave was cooled down to room temperature and a white fluffy powder was collected from the Teflon cylinder. Fourth, the obtained white powder was washed with $0.1 \mathrm{M}$ hydrochloric acid and deionized water $\left(18.2 \mathrm{M} \Omega \cdot \mathrm{cm}\right.$ at $\left.25{ }^{\circ} \mathrm{C}\right)$ alternately until the $\mathrm{pH}$ of the suspension was less than 7. Fifth, the white powder was dried in an oven at $80{ }^{\circ} \mathrm{C}$ overnight and calcined it at a furnace at $700{ }^{\circ} \mathrm{C}$ for $30 \mathrm{~min}$ at a ramp rate of $1{ }^{\circ} \mathrm{C} / \mathrm{min}$. As a result, a dried white powder of pristine single-crystalline anatase $\mathrm{TiO}_{2}$ nanobelts could be collected. Next, the pristine single-crystalline anatase $\mathrm{TiO}_{2}$ nanobelts powder were placed in a quartz-tube furnace, equipped with a gas-flowcontrolled system. The powder was heated at $600{ }^{\circ} \mathrm{C}$ for $3 \mathrm{~h}$ in $\mathrm{H}_{2}$ flow $(0.2 \mathrm{~L} / \mathrm{min})$ at a ramp rate of $10{ }^{\circ} \mathrm{C} / \mathrm{min}$ to form reduced anatase $\mathrm{TiO}_{2}$ nanobelts. The color of the nanobelts became dark blue after this reduction. 


\subsubsection{Characterization of the $\mathrm{TiO}_{2}$ nanobelts}

Five characterization techniques were used for the nanobelts. A field-emission scanning electron microscope (FE-SEM) (JEOL 7600F) was used to observe the morphology of the $\mathrm{TiO}_{2}$ nanobelts. For SEM sampling, $1 \mathrm{mg}$ of the nanobelts was mixed into $5 \mathrm{~mL}$ of deionized water and subsequently sonicated for $1 \mathrm{~min}$ to achieve a homogeneous white suspension. The suspension was dropped onto a piece of silicon wafer substrate $(5 \times 5 \mathrm{~mm}$, SPI Inc.) and dried naturally in air. The dried powder specimens were then ready for SEM observation. The crystal structure of the anatase nanobelts was characterized by a transmission electron microscope (TEM), a highresolution TEM (HRTEM, JEOL JEM 2100F) and X-ray diffraction (XRD, X' Pert Pro PW3040-Pro, Panalytical Inc.) with $\mathrm{Cu} \mathrm{K} \alpha$ radiation. The anatase $\mathrm{TiO}_{2}$ sample was directly placed in a sampling vessel for XRD operation. The sample powder suspension in anhydrous ethanol was dropped onto a holey carbon film supported by a copper grid for TEM. X-ray photoelectron spectroscopy (XPS) experiments were conducted with PHI 5000 Versa Probe system (Physical Electronics, MN). The sample was pressed to a pellet with a diameter around $5 \mathrm{~mm}$. The binding energy of all XPS spectra was calibrated according to the reference of $\mathrm{C} 1 \mathrm{~s}$ peak at $284.8 \mathrm{eV}$. During all XPS measurements, only the electron beam was used to neutralize surface charges rather than argon ions because the argon ions would affect the sample surface and induce oxygen vacancies [28, 29]. $\mathrm{UV}-\mathrm{V}$ is absorption performance was measured under diffuse reflection mode using an integrating sphere (UV2401/2, Shimadzu) attached to a Shimadzu $2550 \mathrm{UV}-\mathrm{Vis}$ spectrometer. The white powder was pressed tightly to a pellet with a diameter of $5-8 \mathrm{~mm}$ in the center of $\mathrm{BaSO}_{4}$ plate. 


\subsubsection{Evaluation of photocatalytic activity of the $\mathrm{TiO}_{2}$ nanobelts}
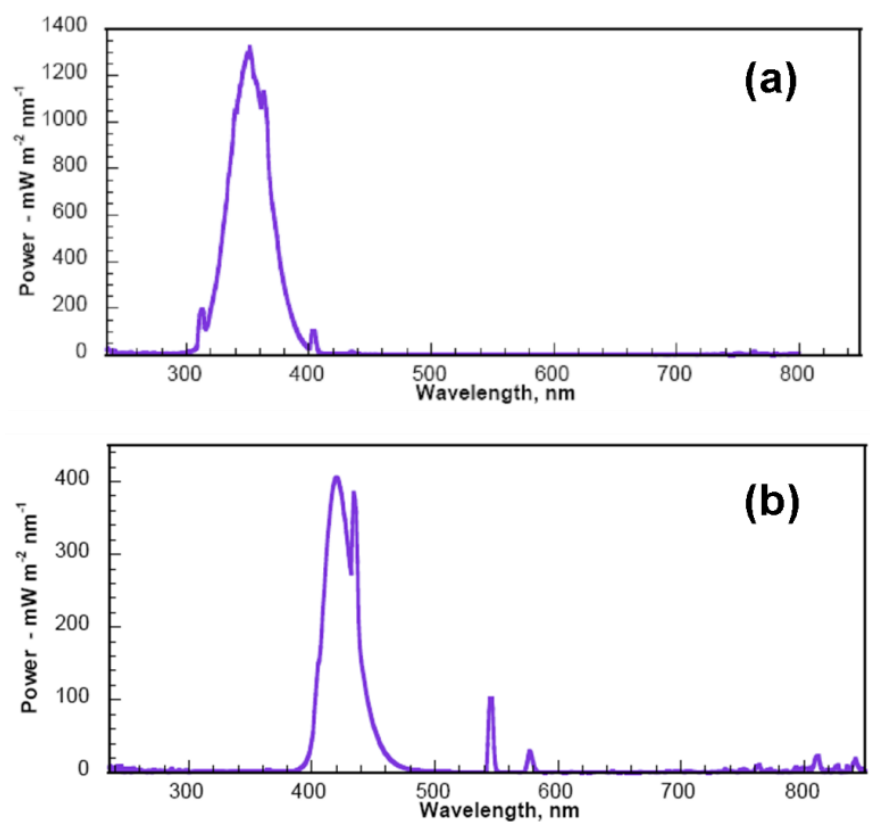

Figure 3-1 Light spectra of LZC-UVA, Luzchem lamps (a) and LZC-420, Luzchem lamps (b).

A commercially obtained photoreactor (LUZ-4 V, Luzchem) with fourteen $8 \mathrm{~W}$ lamps was used in the photocatalytic experiments. Two kinds of lamps were chosen: the first was UV lamps (light spectrum centered at $350 \mathrm{~nm}$, LZC-UVA, Luzchem), the second was visible light lamps (light spectrum centered at $420 \mathrm{~nm}$, LZC-420, Luzchem). The light spectra of the two lamps are shown in Figure 3-1. Decomposition performance of methyl orange was used for evaluating photocatalytic activity of $\mathrm{TiO}_{2}$ nanobelts. 10 mg of $\mathrm{TiO}_{2}$ nanobelts were added into $10 \mathrm{~mL}$ of methyl orange solution (the methyl orange solution was prepared by dissolving methyl orange powder into deionized water with a $\mathrm{pH}$ around 7) in a UV-light-transparent polyethylene tube. The initial methyl orange concentrations were $20 \mathrm{mg} / \mathrm{L}$ and $5 \mathrm{mg} / \mathrm{L}$ for $\mathrm{UV}$ and visible light radiation respectively. The suspensions of methyl orange solution with $10 \mathrm{mg}$ of $\mathrm{TiO}_{2}$ nanobelts 
were sonicated for $0.5 \mathrm{~min}$, preserved in dark for $1 \mathrm{~h}$ to achieve adsorption equilibrium. Then the tubes were placed onto a carousel in the photoreactor to expose every tube to the light evenly. After favorable time intervals, the tubes were taken out from the photoreactor and centrifuged at $10000 \mathrm{rpm}$ for $1.5 \mathrm{~h}$ to separate the supernatant liquid from the bottom catalysts. The liquid was analyzed by UV-Vis spectrometer with a characteristic absorption peak of methyl orange at $464 \mathrm{~nm}$. The intensity of the absorption peak was linearly dependent on the concentration of methyl orange according to Beer's law, so the concentration of methyl orange left after exposure to a fixed time interval of light reflects the photocatalytic rate of $\mathrm{TiO}_{2}$ nanobelts.

\subsection{Results and discussion}

\subsubsection{Morphology and crystal structure of the $\mathrm{TiO}_{2}$ nanobelts}
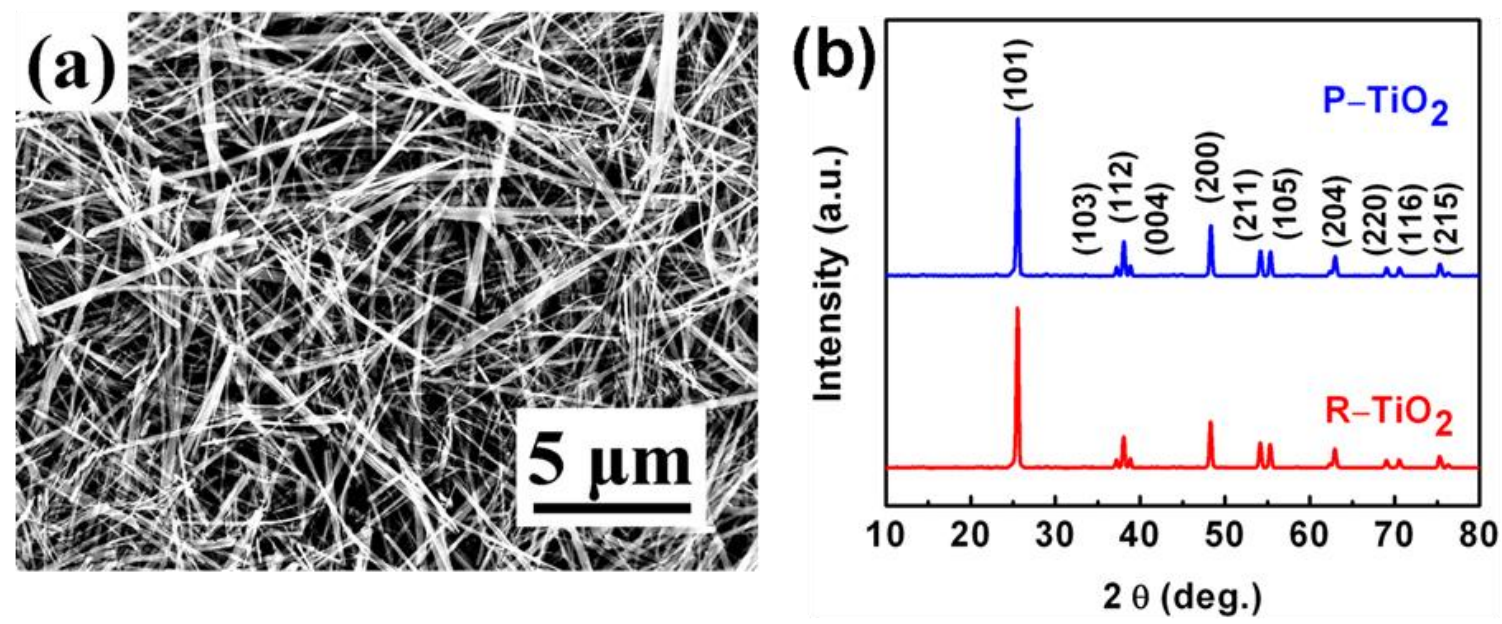

Figure 3-2 (a) SEM image of pristine $\mathrm{TiO}_{2}$ nanobelts; (b) XRD patterns of pristine and reduced $\mathrm{TiO}_{2}$ nanobelts. 
An SEM image of the pristine $\mathrm{TiO}_{2}\left(\mathrm{P}-\mathrm{TiO}_{2}\right)$ nanobelts is shown in Figure 3-2 (a). The hydrothermally prepared $\mathrm{TiO}_{2}$ showed morphology typical of nanobelts. It can be estimated that the width of the nanobelts was below $500 \mathrm{~nm}$ and the length ranged from several micrometers to $15 \mu \mathrm{m}$. The size of the nanobelts synthesized in the present experiment was almost the same as that used in our previous research [7, 16]. In Figure 32 (b), the crystal structure of $\mathrm{TiO}_{2}$ nanobelts did not change after reduction in $\mathrm{H}_{2}$ at 600 ${ }^{\circ} \mathrm{C}$. In other words, the amount of oxygen atoms removed from the $\mathrm{TiO}_{2}$ lattice during reduction process is too little to change the crystal structure. XRD patterns of both the pristine and reduced $\mathrm{TiO}_{2}$ exhibited the monolithic anatase phase. In our previous research literatures, detail crystal structure of the anatase $\mathrm{TiO}_{2}$ nanobelts synthesized with the same method was analyzed by high-resolution transmission electron microscopy [30,31]. Briefly, the $\mathrm{TiO}_{2}$ nanobelts were single-crystalline and grew along the direction of [101], with the dominated facet of (101).

\subsubsection{Chemical status of the $\mathrm{Ti}$ and $\mathrm{O}$ in the $\mathrm{TiO}_{2}$ nanobelts}
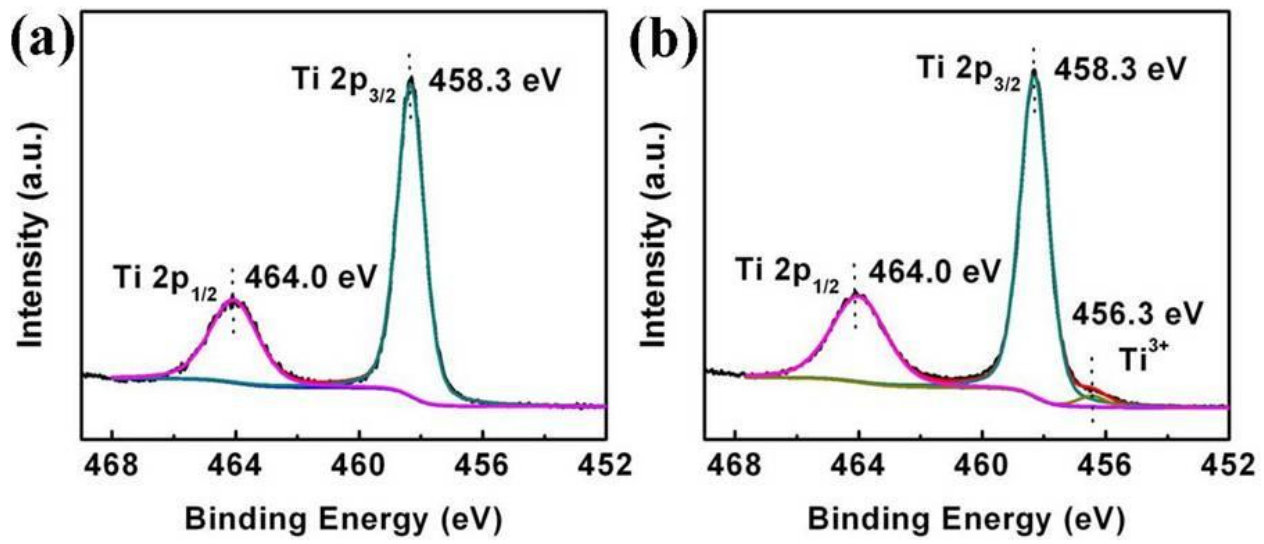

Figure 3-3 XPS spectra of Ti 2p peaks of pristine $\mathrm{TiO}_{2}$ nanobelts (a) and reduced $\mathrm{TiO}_{2}$ nanobelts (b). 

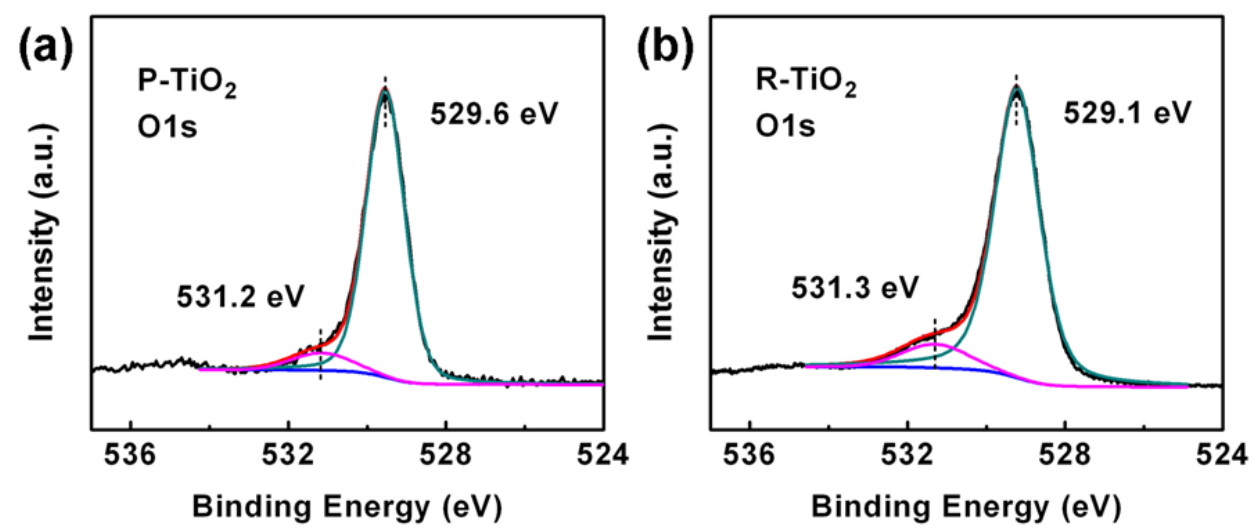

Figure 3-4 XPS spectra of $\mathrm{O} 1 \mathrm{~s}$ peaks of pristine $\mathrm{TiO}_{2}$ nanobelts (a) and reduced $\mathrm{TiO}_{2}$ nanobelts (b).

XPS measurements were performed on both the pristine and reduced $\mathrm{TiO}_{2}$ nanobelts. Figure 3-3 shows XPS spectra of the Ti 2p core levels. Both the pristine and reduced $\mathrm{TiO}_{2}$ exhibited a $\mathrm{Ti} 2 \mathrm{p} 3 / 2$ peak at $458.3 \mathrm{eV}$ and a $\mathrm{Ti} 2 \mathrm{p} 1 / 2$ peak at $464.0 \mathrm{eV}$, with a standard energy difference of $5.7 \mathrm{eV}$ [32]. It is also shown that there were no changes of the Ti $2 p$ peak before and after reduction. However, a peak of the reduced $\mathrm{TiO}_{2}$ nanobelts was bumped up at $456.3 \mathrm{eV}$ in Figure 3-3 (b). This small peak at lower binding energy level was attributed to $2 p$ status of $\mathrm{Ti}^{3+}$, which proves the existence of oxygen vacancy in $\mathrm{TiO}_{2}$ lattice [33]. During the XPS measurements, the argon ion gun was not used for neutralizing surface charges, so the oxygen vacancy could not be generated by the XPS measurement. In other words, the oxygen vacancy was produced in the reduction procedure exclusively. Based on our XPS analysis, it could be calculated that the $\mathrm{Ti}^{3+}$ content was 3.4 at. $\%$. Accordingly, the content of oxygen vacancy was 0.85 at. \%. Parallel shifting of the $\mathrm{O} 1 \mathrm{~s}$ peak to a lower binding energy level is another direct evidence of the oxygen vacancy $[7,34]$. In the present experiments, the $\mathrm{O} 1 \mathrm{~s}$ peak of $\mathrm{TiO}_{2}$ nanobelts was shifted from $529.6 \mathrm{eV}$ to $529.1 \mathrm{eV}$ after reduction treatment in Figure 
3-4. The shift of $\mathrm{O} 1 \mathrm{~s}$ binding energy was similar as the result in our previous experiments of doping nitrogen into anatase [7]. The difference of binding energy shift in these two experiments was probably due to different amount of oxygen vacancies induced into the $\mathrm{TiO}_{2}$ lattice. It was demonstrated that chemical status of the oxygen vacancy generated in reduction process was almost the same as that from nitrogen doping. In Figure 3-4, another two $\mathrm{O} 1 \mathrm{~s}$ peaks were located at $531.2 \mathrm{eV}$ and $531.3 \mathrm{eV}$ respectively. These two peaks with almost the same binding energy were resulted from hydroxides of moisture adsorbed on the $\mathrm{TiO}_{2}$ surface [32].

\subsubsection{Light absorption of the $\mathrm{TiO}_{2}$ nanobelts}

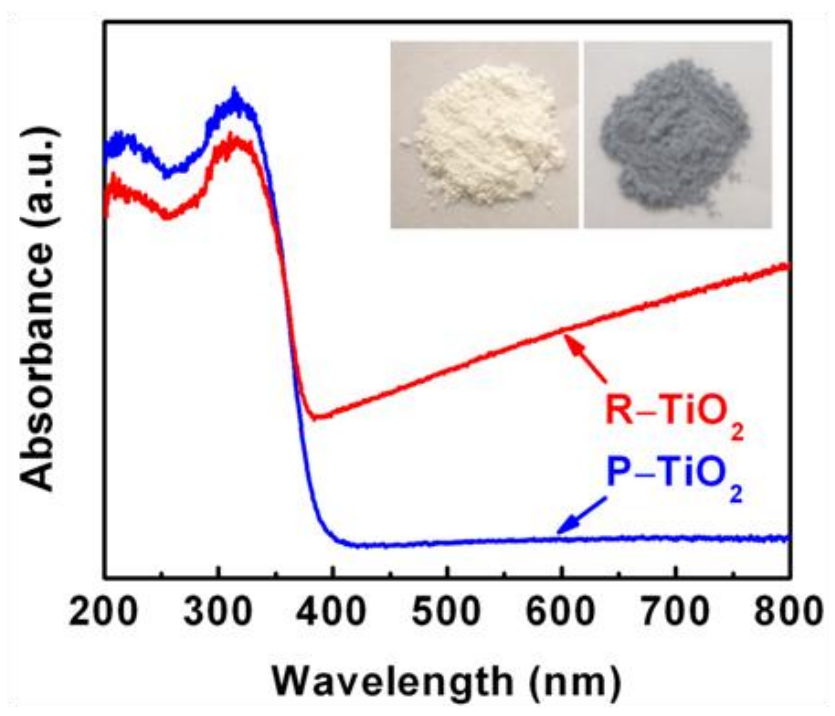

Figure 3-5 Absorbance spectra of the pristine $\mathrm{TiO}_{2}$ nanobelts and the reduced $\mathrm{TiO}_{2}$ nanobelts. The insets are the optical photos of the pristine $\mathrm{TiO}_{2}$ (left) and reduced $\mathrm{TiO}_{2}$ (right), which shows the color change from white to blue due to reduction in $\mathrm{H}_{2}$ flow at $600^{\circ} \mathrm{C}$. 
Figure 3-5 shows the UV-Vis absorbance curves of the pristine $\mathrm{TiO}_{2}$ nanobelts and reduced $\mathrm{TiO}_{2}$ nanobelts. The blue spectrum was the typical anatase $\mathrm{TiO}_{2}$ absorbance curve with optical absorption edge at $385 \mathrm{~nm}$, which corresponds to an optical band gap of $3.2 \mathrm{eV}$. The red spectrum is of the reduced anatase $\mathrm{TiO}_{2}$ and had the same absorption edge at $385 \mathrm{~nm}$, which meant the band gaps of the pristine and reduced $\mathrm{TiO}_{2}$ were identical. The reduced $\mathrm{TiO}_{2}$, however, had an up trending absorption tail in the visible light range, which means the visible-light-absorption of reduced $\mathrm{TiO}_{2}$ was increased. This is easily seen by the color change from white (inset of Figure 3-5) to blue after reduction process in $\mathrm{H}_{2}$ flow at $600{ }^{\circ} \mathrm{C}$. Although doping nitrogen ions or other nonmetal ions can also change the color of $\mathrm{TiO}_{2}$, the color change resulted from oxygen vacancy is completely different $[7,35]$. As nitrogen atoms were doped into $\mathrm{TiO}_{2}$, the $\mathrm{N} 2 \mathrm{p}$ state could be hybridized with $\mathrm{O} 2 \mathrm{p}$ state, which added an absorption shoulder onto the band edge $[35,36]$. However, the valence band structures of the pristine and reduced $\mathrm{TiO}_{2}$ were identical without add-on shoulders, because there were no dopants in $\mathrm{TiO}_{2}$. Herein, the color change was attributed to the oxygen vacancy and associated $\mathrm{Ti}^{3+}$ species, which could act as color centers [37]. 


\subsubsection{Photocatalytic activities of the $\mathrm{TiO}_{2}$ nanobelts}
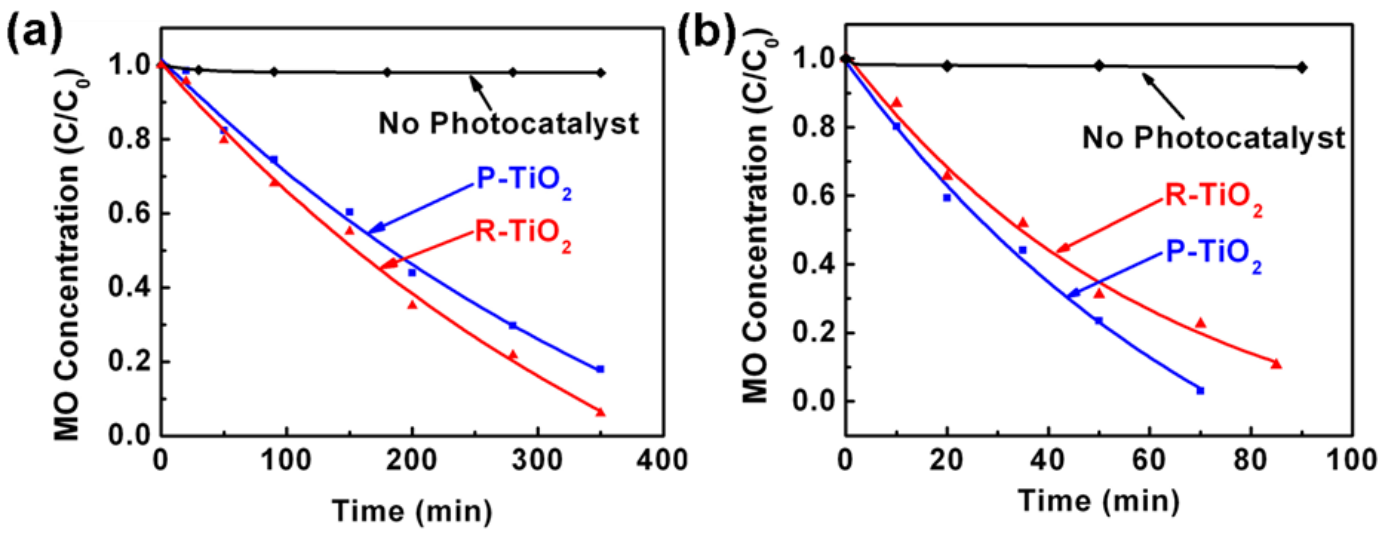

Figure 3-6 Photocatalytic activities of the pristine $\mathrm{TiO}_{2}$ nanobelts $\left(\mathrm{P}-\mathrm{TiO}_{2}\right)$ and the reduced $\mathrm{TiO}_{2}\left(\mathrm{R}-\mathrm{TiO}_{2}\right)$ under visible light irradiation (a), and under UV light irradiation (b).

The evaluation of photocatalytic activities of the $\mathrm{TiO}_{2}$ nanobelts was conducted by monitoring the concentration change of methyl orange (MO) aqueous solution with $\mathrm{TiO}_{2}$ nanobelts as the photocatalyst under visible light and UV light irradiation. The more methyl orange decomposed in the fixed time interval, the higher the photocatalytic activity. Methyl orange aqueous solution without any photocatalyst was used as a reference under both UV and visible light irradiation to ensure no photodegradation was present. It is clearly shown in Figure 3-6 that methyl orange was only decomposed slightly under UV light and visible light without photocatalysts in the solutions. Figure 36 (a) and (b) show the degradation curves of methyl orange with $\mathrm{TiO}_{2}$ nanobelts in visible light and UV light, respectively. When exposed in visible light, as in Figure 3-6 (a), the reduced $\mathrm{TiO}_{2}$ nanobelts had higher photocatalytic activity than the pristine $\mathrm{TiO}_{2}$ nanobelts. In Figure 3-5 (b), when exposed in UV light, the photocatalytic activity of the reduced $\mathrm{TiO}_{2}$ nanobelts was lower than the pristine $\mathrm{TiO}_{2}$ nanobelts. The photocatalytic 
activities of the pristine $\mathrm{TiO}_{2}$ and the reduced $\mathrm{TiO}_{2}$ in UV light were completely reversed compared with that in visible light. The decomposition rates of methyl orange in UV light were higher than that in visible light for the both pristine and reduced $\mathrm{TiO}_{2}$ nanobelts.

\subsection{Conclusions}

These results confirm that the oxygen vacancy defects in anatase $\mathrm{TiO}_{2}$ play both a positive and a negative role for photocatalysis, and that ion doping does not always beneficial to the photocatalytic performance. In visible light, the light absorption of the

reduced $\mathrm{TiO}_{2}$ was higher than the pristine $\mathrm{TiO}_{2}$, increasing visible-light photocatalysis. However, in UV light range, there was no increase in absorption but the photocatalysis decreased in the reduced sample. This shows that the oxygen vacancies must be acting as trap states and decreasing the yield of photoexcited carriers, decreasing the overall performance. Since oxygen vacancies are almost always produced when $\mathrm{TiO}_{2}$ is doped, these results are important for guiding the selection of dopants and design of oxygen vacancy based photocatalysts.

\section{Reference}

[1] Tracy, L.T.; Yates, J. T. Chem. Rev. 2006, 106, 4428-4453.

[2] Linsebigler, A. L.; Lu, G. Q.; Yates, J. T. Chem. Rev. 1995, 95, 735-758.

[3] Fox, M. A.; Dulay, M. T. Chem. Rev. 1993, 93, 341-357.

[4] Hoffmann, M. R.; Martin, S. T.; Choi, W.; Bahnemannt, D. W. Chem. Rev. 1995, 95, 69-96. 
[5] Hagfeldtt, A.; Gratzel, M. Chem. Rev. 1995, 95, 49-68.

[6] Kalyanasundaram, K.; Gratzel, M. Coord. Chem. Rev. 1998, 77, 347-414.

[7] Wang, J.; Tafen, D. N.; Lewis, J. P.; Hong, Z. L.; Manivannan, A.; Zhi, M. J.; Li, M.;Wu, N. Q. J. Am. Chem. Soc. 2009, 131, 12290-12297.

[8] Yamashita, H.; Harada, M.; Misaka, J.; Takeuchi, M.; Ichihashi, Y.; Goto, F.; Ishida, M.; Sasaki, T.; Anpo, M. J. Synchrotron. Rad. 2001, 8, 569-571.

[9] Sasikala, R.; Sudarsan, V.; Sudakar, C.; Naik, R.; Sakuntala, T.; Bharadwaj, S. R. Int. J. Hydrog. Energy. 2008, 33, 4966-4973.

[10] Zhu, H.; Tao, J.; Dong, X. J. Phys. Chem. C. 2009, 114, 2873-2879.

[11] Yu, H. G.; Irie, H.; Hashimoto, K. J. Am. Chem. Soc. 2010, 132, 6898-6899.

[12] Gai, Y. Q.; Li, J. B.; Li, S. S.; Xia, J. B.; Wei, S. H. Phys. Rev. Lett. 2009, 102, 036402(4).

[13] Lindgren, T.; Mwabora, J. M.; Avendano, E.; Jonsson, J.; Hoel, A.; Granqvist, C. G.; Lindquist, S. E. J. Phys. Chem. B. 2003, 107, 5709-5716.

[14] Hamal, D. B.; Haggstrom, J. A.; Marchin, G. L.; Ikenberry, M. A.; Hohn, K.; Klabunde, K. J.; Langmuir. 2010, 26, 2850-2810.

[15] Tafen, D. N.; Wang, J.; Wu, N. Q.; Lewis, J. P. Appl. Phys. Lett. 2009, 94, 093101.

[16] Wu, N. Q.; Wang, J.; Tafen, D. N.; Wang, H.; Zheng, J. G.; Lewis, J. P.; Liu, X. G.; Leonard, S. S.; Manivannan, A. J. Am. Chem. Soc. 2010, 132, 6679-6685.

[17] Jiang, Z.; Yang, F.; Luo, N. J.; Chu, B. T. T.; Sun, D. Y.; Shi, H. H.; Xiao, T. C.; Edwards, P. P. Chem. Commun. 2008, 47, 6372-6374.

[18] Chen, X. B.; Burda, C. J. Am. Chem. Soc. 2008, 130, 5018-5019.

[19] Tao, J. G.; Batzill, M. J. Phys. Chem. L. 2010, 1, 3200-3206. 
[20] Dulub, O.; Batzill, M.; Solovev, S.; Loginova, E.; Alchagirov, A.; Madey, T. E.; Diebold, U. Science, 2007, 317, 1052-1056.

[21] Wendt, S.; Sprunger, P. T.; Lira, E.; Madsen, G. K. H.; Li, Z. S.; Hansen, J.; Matthiesen, J.; Rasmussen, A. B.; Laegsgaard, E.; Hammer, B. et al. Science, 2008, 320, $1755-1759$.

[22] Linsebigler, A.; Lu, G. Q.; Yates, J. T. J. Chem. Phys. 1995, 103, 9438-9443.

[23] Varghese, O. K.; Gong, D. W.; Paulose, M.; Ong, K. G.; Dickey, E. C.; Grimes, C. A. Adv. Mater. 2003, 15, 624-627.

[24] Wu, N. Q.; Zhao, M. H.; Zheng, J. G.; Jiang, G. B.; Myers, B.; Li, S. X.; Chyu, M.; Mao, S. M. Nanotechnology 2005, 16, 2878-2881.

[25] Tan, B.; Wu, Y. Y. J. Phys. Chem. B. 2006, 110, 15932-15938.

[26] Zhu, K.; Neale, N. R.; Miedaner, A.; Frank, A. J. Nano Lett. 2007, 7, 69-74.

[27] Kudo, A.; Miseki, Y. Chem. Soc. Rev. 2009, 38, 253-278.

[28] Olojo, R. O.; Xia, R. H.; Abramson, J. J. Anal. Biochem. 2005, 339, 338-344.

[29] Barry, H.; Matthew, W. Br. J. Pharmacol. 2004, 142, 231-255.

[30] Setsukinai, K.; Urano, Y.; Kakinuma, K.; Majima, H. J.; Nagano, T. J. Biol. Chem. 2003, 278, 3170-3175.

[31] Tachikawa, T.; Majima, T. Langmuir 2009, 25, 7791-7802.

[32] Moulder, J. F.; Stickle, W. F.; Sobol, P. E.; Bomben, K. D. Handbook of X-ray Photoelectron Spectroscopy; Eden Prairie: 1992.

[33] He, Y. B.; Dulub, O.; Cheng, H. Z.; Selloni, A.; Diebold, Ulrike. Phys. Rev. Lett. 2009, 102, 106105(4). 
[34] Rumaiz, A. K.; Ali, B.; Ceylan, A.; Boggs, M.; Beebe, T.; Shah, S. I. Solid State Commun. 2007, 144, 334-338.

[35] Chen, X. B.; Burda, C. J. Am. Chem. Soc. 2008, 130, 5018-5019.

[36] Asahi, R.; Morikawa, T.; Ohwaki, T.; Aoki, K.; Taga, Y. Science, 2001, 293, 269271.

[37] Sekiya, T.; Ichimura, K.; Igarashi, M.; Kurita, S. J. Phys. Chem. Solids. 2000, 61, $1237-1242$. 


\section{Chapter 4 Visible Light Photocatalytic Activity of Nitrogen- Doped $\mathrm{La}_{2} \mathrm{Ti}_{2} \mathrm{O}_{7}$ Nanosheets Originating from Band Gap \\ Narrowing}

\subsection{Background and introduction}

As we discussed above, semiconductor photocatalysts have attracted considerable attention from scientists and engineers owing to their broad potential applications in solar energy conversion, medical photodynamic therapy and environmental remediation [1-4]. However, significant challenges remain in developing semiconductor photocatalysts. These challenges include the limited absorption of visible light and infrared light, low mobility of charge carriers and high recombination rate of electron-hole pairs in previously reported materials. As shown in section 3, anatase titanium dioxide $\left(\mathrm{TiO}_{2}\right)$ is the most extensively used photocatalyst [5]. However, its large band gap (3.2 eV) only allows the absorption of ultraviolet (UV) light, which accounts less than $5 \%$ of natural solar radiation [6]. In order to develop visible light responsive photocatalysts, intense efforts are being focused on doping ions into anatase $\mathrm{TiO}_{2}$. Either anion [6-8] or cation [9, 10] doping induces localized states between the valence band and the conduction band, as shown in Chapter 3. The charge carriers created in such mid-gap states exhibit poor mobility and high recombination rate of the electron-hole pairs. Hence doping the anatase $\mathrm{TiO}_{2}$ lattice with ions usually leads to limited visible light photocatalytic activity and even has an adverse effect on the UV light photocatalytic activity [10]. Therefore if 
doping is to lead to a net increase in photocatalytic activity, the dopants must form a continuum with the valence or conduction band edge and not mid gap states.

The results of oxygen vacancies in Chapter 3 show the electronic band structure plays a critical role when considering dopants. In this chapter, we therefore switch from $\mathrm{TiO}_{2}$ to $\mathrm{La}_{2} \mathrm{Ti}_{2} \mathrm{O}_{7}$ which is a member of a family of the perovskite-type layer-structured photocatalysts (such as $\mathrm{Sr}_{3} \mathrm{Ti}_{2} \mathrm{O}_{7}, \mathrm{~K}_{2} \mathrm{La}_{2} \mathrm{Ti}_{3} \mathrm{O}_{10}$ and $\mathrm{La}_{2} \mathrm{Ti}_{2} \mathrm{O}_{7}$ ) [11-13]. $\mathrm{La}_{2} \mathrm{Ti}_{2} \mathrm{O}_{7}$ has periodically arranged corner-shared $\mathrm{TiO}_{6}$ octahedrons and La cations [14]. In such a structure, the mobility of charge carriers is boosted by the network of octahedrons [14], leading to high quantum yields especially for photocatalytic water splitting $[4,12,13]$. However, its large band gap is still a major technical barrier for practical applications [15-17]. We show that by doping $\mathrm{La}_{2} \mathrm{Ti}_{2} \mathrm{O}_{7}$ with nitrogen a continuum of states is formed instead of mid-gap trap states and a net increase in overall photocatalysis is gained without the losses seen in reduced $\mathrm{TiO}_{2}$ in Chapter 3 . This is achieved by using anion instead of cation doping, since cation doping only induces donor bands (i.e., mid-gap states) between the valence band and the conduction band $[14,18,19]$.

Another technical barrier in the development of the $\mathrm{La}_{2} \mathrm{Ti}_{2} \mathrm{O}_{7}$ photocatalysts is the lack of an effective synthesis approach. Currently solid-state reaction (SSR) is the commonly used method for the synthesis of perovskite $\mathrm{La}_{2} \mathrm{Ti}_{2} \mathrm{O}_{7}$ photocatalysts $[12,18$, 20-22]. It requires high temperatures $\left(>1100{ }^{\circ} \mathrm{C}\right)$ and usually generates micron-size particles that have a very low specific surface area $[14,23]$. Here we employ a hydrothermal method to synthesize $\mathrm{La}_{2} \mathrm{Ti}_{2} \mathrm{O}_{7}$ photocatalysts because such an approach is simple, inexpensive and able to produce large quantities of nanomaterials at a lowtemperature $\left(<260{ }^{\circ} \mathrm{C}\right)[16,24]$. Here we also demonstrate the ability to synthesize 
nitrogen-doped $\mathrm{La}_{2} \mathrm{Ti}_{2} \mathrm{O}_{7}$ nanosheets, a two-dimensional (2-D) nanostructure. Our previous report has shown that one-dimensional (1-D) and 2-D nanostructures exhibit higher mobility and lower recombination rates of charge carriers than zero-dimensional (0-D) nanoparticles [25]. Therefore, 1-D and 2-D nanostructures show better photocatalytic activity than $0-\mathrm{D}$ counterparts.

In this chapter, an effective method is developed to synthesize nitrogen-doped $\mathrm{La}_{2} \mathrm{Ti}_{2} \mathrm{O}_{7}$ nanosheets with excellent visible light photocatalytic activity. The band gap and the light absorption properties of the nanosheets are studied. Furthermore, the photocatalytic activity of the resulting material is evaluated under both ultraviolet and visible light irradiation. Finally, the correlation of the band structure with the photocatalytic performance is discussed.

\subsection{Experimental section}

\subsubsection{Chemicals and materials}

Lanthanum(III) nitrate hexahydrate $\left(\mathrm{La}\left(\mathrm{NO}_{3}\right)_{3} \cdot 6 \mathrm{H}_{2} \mathrm{O}, \geq 99.0 \%\right.$, particles), sodium hydroxide $(\mathrm{NaOH}, \geq 98 \%$, pellets $)$ and anatase titanium dioxide $\left(\mathrm{TiO}_{2}, \geq 99.8 \%\right.$, powder $)$ were purchased from Sigma-Aldrich. Titanium sulfate nonahydrate $\left(\mathrm{Ti}\left(\mathrm{SO}_{4}\right)_{2} \cdot 9 \mathrm{H}_{2} \mathrm{O}\right)$ was purchased from Chem Service, Inc.. The resistivity of deionized water was $18.2 \mathrm{M} \Omega \cdot \mathrm{cm}$ at $25^{\circ} \mathrm{C}$. 


\subsubsection{Synthesis of $\mathrm{La}_{2} \mathrm{Ti}_{2} \mathrm{O}_{7}$ nanosheets}

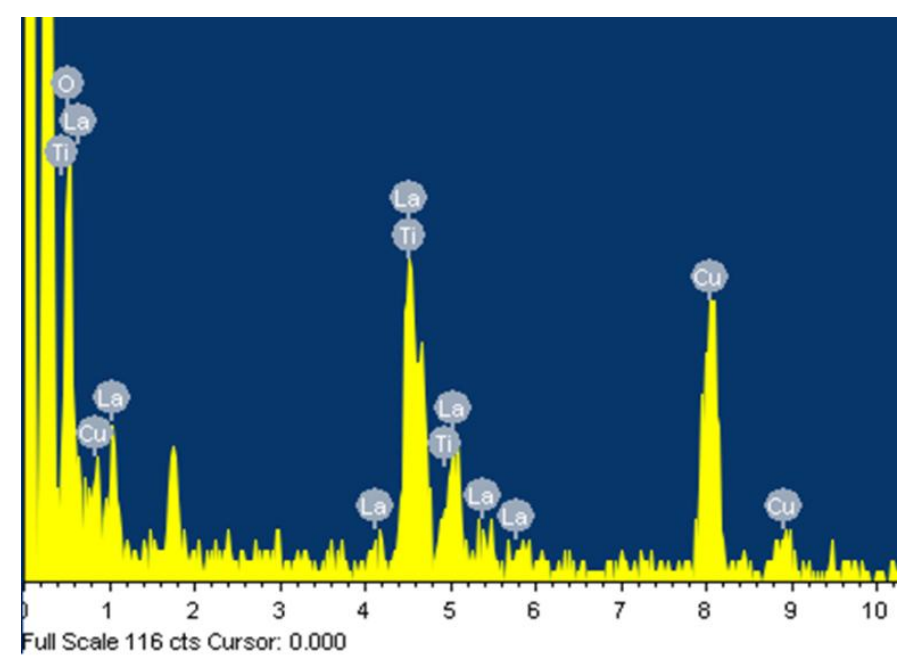

Figure 4-1 Energy-dispersive X-ray spectroscopy (EDX) spectrum obtained from the pristine $\mathrm{La}_{2} \mathrm{Ti}_{2} \mathrm{O}_{7}$ after hydrothermal processing

The nitrogen-doped $\mathrm{La}_{2} \mathrm{Ti}_{2} \mathrm{O}_{7}$ nanosheets were synthesized in several steps. First, $0.5 \mathrm{~g}$ of $\mathrm{Ti}\left(\mathrm{SO}_{4}\right)_{2} \cdot 9 \mathrm{H}_{2} \mathrm{O}$ and $0.9 \mathrm{~g}$ of $\mathrm{La}\left(\mathrm{NO}_{3}\right)_{3} \cdot 6 \mathrm{H}_{2} \mathrm{O}$ were added to $20 \mathrm{~mL}$ of $1.0 \mathrm{~mol} / \mathrm{L}$ $\mathrm{NaOH}$ aqueous solution. The mixture was stirred vigorously in an ultrasonic bath for 30 min and then transferred to a $30 \mathrm{~mL}$ Teflon-lined nickel alloy autoclave. The autoclave was sealed and then heated at $240{ }^{\circ} \mathrm{C}$ for $24 \mathrm{~h}$. After hydrothermal treatment, the autoclave was cooled down to room temperature naturally. A white fluffy powder was obtained. The collected product was then washed with copious amounts of deionized water and centrifuged several times until the $\mathrm{pH}$ of the solution reached 7 . The product was subsequently dried in air at $80{ }^{\circ} \mathrm{C}$ overnight. The white powder contained only $\mathrm{La}$, $\mathrm{Ti}$ and $\mathrm{O}$ elements as confirmed by the energy-dispersive X-ray spectroscopy (EDX) spectrum in shown as Figure 4-1. Next, the white sample was heated in $\mathrm{NH}_{3}$ flow in a 
tubular furnace equipped with a gas flow meter to $600{ }^{\circ} \mathrm{C}$ at a ramp rate of $5{ }^{\circ} \mathrm{C} / \mathrm{min}$, and then held at $600{ }^{\circ} \mathrm{C}$ in $\mathrm{NH}_{3}$ at a flow rate of $20 \mathrm{~mL} / \mathrm{min}$ for $8 \mathrm{~h}$ in order to dope nitrogen into the $\mathrm{La}_{2} \mathrm{Ti}_{2} \mathrm{O}_{7}$ nanosheets. After heat treatment in $\mathrm{NH}_{3}$ flow, the sample was cooled down to room temperature in $\mathrm{N}_{2}$ flow at a rate of $5{ }^{\circ} \mathrm{C} / \mathrm{min}$. The sample became yellow after $\mathrm{NH}_{3}$ treatment.

\subsubsection{Characterization of the $\mathrm{La}_{2} \mathrm{Ti}_{2} \mathrm{O}_{7}$ nanosheets}

The structure and morphology of the $\mathrm{La}_{2} \mathrm{Ti}_{2} \mathrm{O}_{7}$ nanosheets was characterized using a transmission electron microscope (TEM), a high-resolution TEM (HRTEM, JEOL JEM 2100F) and X-ray diffraction (XRD, X'Pert PRO PW3040-Pro, PANalytical Inc.) with $\mathrm{Cu} \mathrm{Ka}$ radiation. For TEM sample preparation, the powders were suspended in ethanol. A drop of suspension was deposited onto a holey carbon film supported by a copper grid. The specimen was dried in air prior to TEM examination. The morphology was observed with a field-emission scanning electron microscope (FE-SEM) (JEOL 7600F) equipped with an energy-dispersive X-ray spectrometer (EDX) and an atomic force microscope (AFM) (Pico Scan 3000). For SEM sample preparation, $1 \mathrm{mg}$ of $\mathrm{La}_{2} \mathrm{Ti}_{2} \mathrm{O}_{7}$ nanosheets was mixed with $2 \mathrm{~mL}$ of deionized water in a $5 \mathrm{~mL}$ sample vial and then sonicated in an ultrasonic bath for $10 \mathrm{~s}$ to achieve a suspension. The suspension was then dropped onto a smooth silicon wafer substrate (SPI Inc.) in air. For AFM sample preparation, the suspension was prepared in a similar way except for the use of mica as the substrate. The $\mathrm{La}_{2} \mathrm{Ti}_{2} \mathrm{O}_{7}$ nanosheets were also characterized by X-ray photoelectron spectroscopy (XPS) (PHI 5000 Versa Probe system, Physical Electronics, MN). The C 1s peak at $284.8 \mathrm{eV}$ was used as a reference for calibrating the binding energy of the XPS spectra. The UV- 
visible absorption spectra were obtained in the diffuse reflectance mode with a Shimadzu 2550 UV-visible spectrometer equipped with an integrating sphere (UV 2401/2, Shimadzu). The sample powder was pressed to form a pellet and placed on a $\mathrm{BaSO}_{4}$ plate. The specific surface area of the nanosheets was determined using the Brunauer-EmmettTeller (BET) method.

\subsubsection{Photocatalysis testing of the $\mathrm{La}_{2} \mathrm{Ti}_{2} \mathrm{O}_{7}$ nanosheets}

A commercial photoreactor (LUZ-4V, Luzchem) equipped with fourteen $8 \mathrm{~W}$ visible (Luzchem LZC- 420) or ultraviolet (Luzchem LZC-UVA) lamps was used for visible and UV photocatalysis experiments, respectively. $10 \mathrm{mg}$ of $\mathrm{La}_{2} \mathrm{Ti}_{2} \mathrm{O}_{7}$ nanosheets were added to $10 \mathrm{~mL}$ of a solution of $5 \mathrm{mg} / \mathrm{mL}$ of methyl orange (MO) in a $10 \mathrm{~mL}$ polyethylene tube. The MO solution was prepared by dissolving MO powder into deionized water with a $\mathrm{pH}$ around 7. For each photocatalytic experiment, the tubes containing the suspensions were sonicated in the dark for $1 \mathrm{~min}$, and then kept in the dark for $2 \mathrm{~h}$ to reach surface adsorption equilibrium. Then the tubes were mounted onto the carousel inside the photoreactor to ensure the even exposure of each tube to the light. At different time intervals of irradiation, the tubes were unloaded and centrifuged at 5000 $\mathrm{rpm}$ for $1 \mathrm{~h}$ to separate the supernatant liquid from the catalysts. The supernatant liquid was collected and analyzed by recording the characteristic absorption of methyl orange (464 nm) using the UV-visible spectrometer. According to the calibration plot of the UV absorbance as a function of the remaining methyl orange concentration, the efficiency of the methyl orange decomposition was calculated. As a reference, methyl orange solution without any photocatalyst was used as a control sample in all photocatalysis experiments. 


\subsection{Results and discussion}

\subsubsection{Morphology and crystal structure}
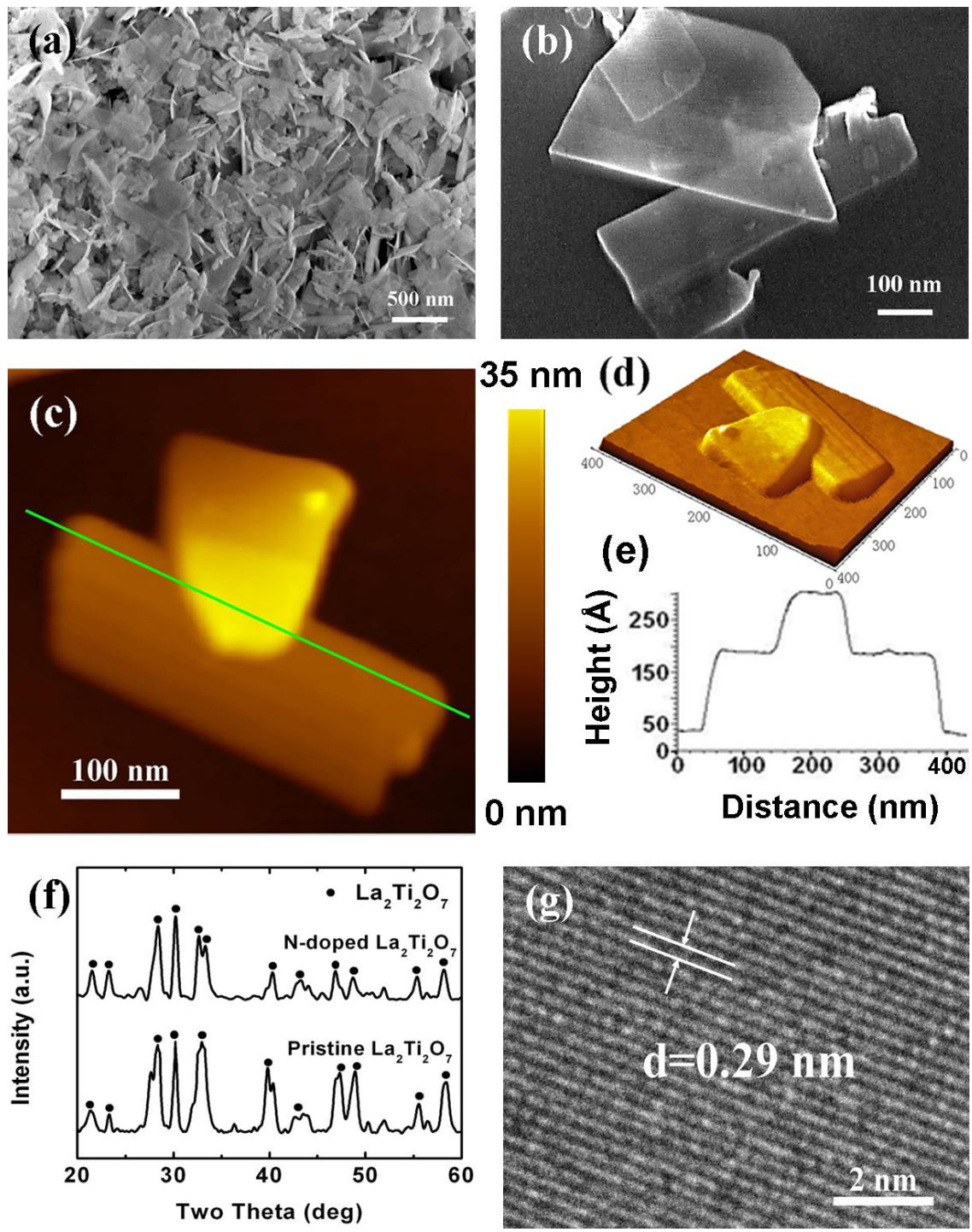

Figure 4-2 Images of the pristine $\mathrm{La}_{2} \mathrm{Ti}_{2} \mathrm{O}_{7}$ nanosheets including $\mathrm{SEM}$ images at low (a) and high (b) magnification, AFM images in two-dimension (c), three-dimensions (d) and height profile analysis (e) along the green line in (c), XRD patterns obtained before and after nitrogen doping (f), and a HRTEM image (g). 
Figure 4-2 (a) shows the accumulation of the $\mathrm{La}_{2} \mathrm{Ti}_{2} \mathrm{O}_{7}$ nanosheets deposited on a silicon wafer substrate. Most of the nanosheets were between $100 \mathrm{~nm}$ and $500 \mathrm{~nm}$ in size. A high-magnification SEM image (Figure 4-2 (b)) shows two pieces of $\mathrm{La}_{2} \mathrm{Ti}_{2} \mathrm{O}_{7}$ nanosheets stacked together. To examine the thickness of the nanosheets, AFM was used to image individual pieces (Figures 4-2 (c) and 1(d)). The AFM image reveals that the surface of the nanosheets was very smooth. The profile analysis in Figure 4-2 (e) shows that the thicknesses of the top and bottom $\mathrm{La}_{2} \mathrm{Ti}_{2} \mathrm{O}_{7}$ nanosheets were 12 and $15 \mathrm{~nm}$, respectively.

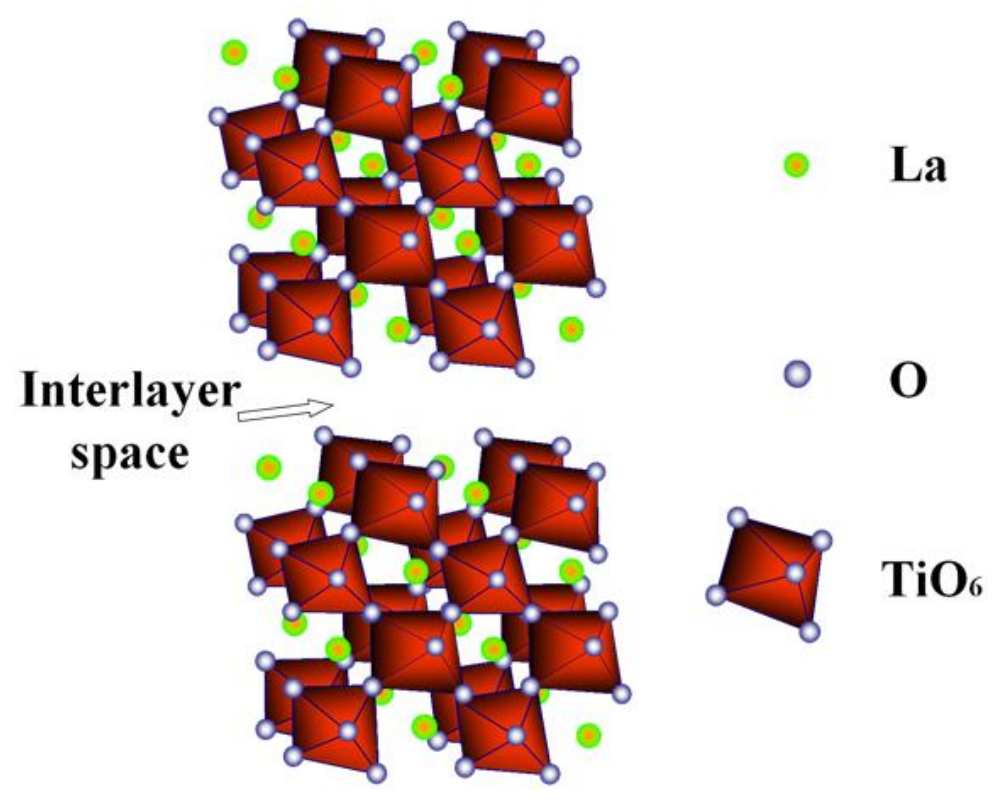

Figure 4-3 Schematic of perovskite structure of $\mathrm{La}_{2} \mathrm{Ti}_{2} \mathrm{O}_{7}$

The XRD pattern of the pristine $\mathrm{La}_{2} \mathrm{Ti}_{2} \mathrm{O}_{7}$ nanosheets (Figure 4-2 (f)) confirmed that the nanosheets had a monoclinic structure with space group P21 formed by alternating perovskite-like blocks of nTiO6 octahedron slabs (as shown in Figure 4-3). HRTEM observation revealed that individual $\mathrm{La}_{2} \mathrm{Ti}_{2} \mathrm{O}_{7}$ nanosheets were single-crystals 
(Figure 4-2 (g)). This is one of the advantages of our synthesis method over the conventional SSR method, since SSR processing usually leads to a polycrystalline structure. A single-crystal structure is highly desirable for photocatalysis since the grain boundaries typically act as the sites for recombination of photogenerated electron-hole pairs [25]. The HRTEM image in Figure 4-2 (g) shows lattice fringes with a spacing of $0.29 \mathrm{~nm}$, which corresponds to the (212) spacing of $\mathrm{La}_{2} \mathrm{Ti}_{2} \mathrm{O}$ 7. It is also worth noting that the two predominant surfaces of the $\mathrm{La}_{2} \mathrm{Ti}_{2} \mathrm{O}_{7}$ nanosheets were the (202) facets.

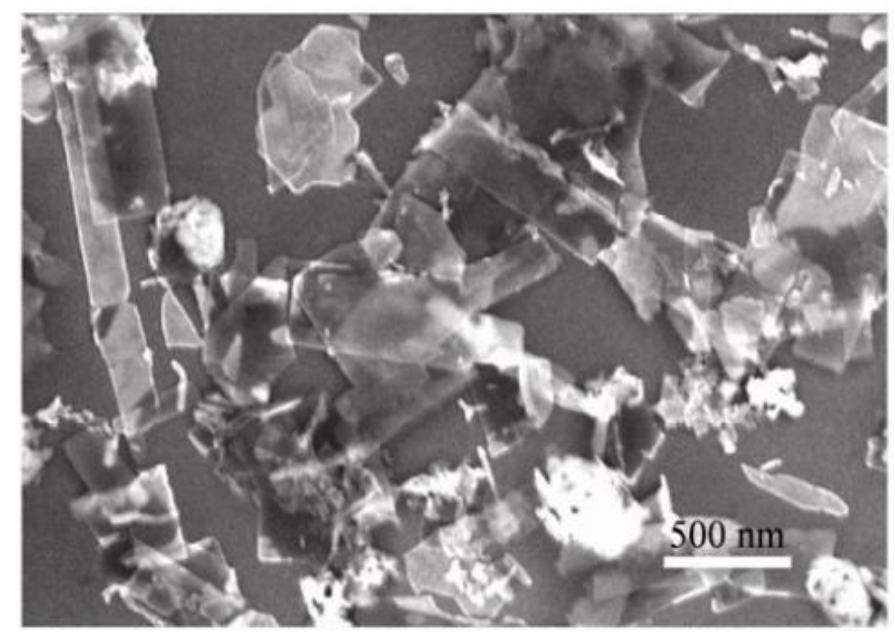

Figure 4-4 SEM image of the nitrogen-doped $\mathrm{La}_{2} \mathrm{Ti}_{2} \mathrm{O}_{7}$ nanosheets

The shape and the dimension of the nanosheets were retained after nitrogen doping (Figure 4-4 in the ESM). The BET specific surface area of the N-doped $\mathrm{La}_{2} \mathrm{Ti}_{2} \mathrm{O}_{7}$ nanosheets was determined to be $38 \mathrm{~m}^{2} / \mathrm{g}$, which is much larger than that of the $\mathrm{La}_{2} \mathrm{Ti}_{2} \mathrm{O}_{7}$ sample synthesized by the SSR method $\left(1-2 \mathrm{~m}^{2} / \mathrm{g}\right)$ [26]. After heat treatment in $\mathrm{NH}_{3}$ flow, the crystal phase did not change, which was confirmed by the XRD pattern in Figure 4-2 (f). No impurities or new phases were found. 


\subsubsection{Chemical state and electronic structure}
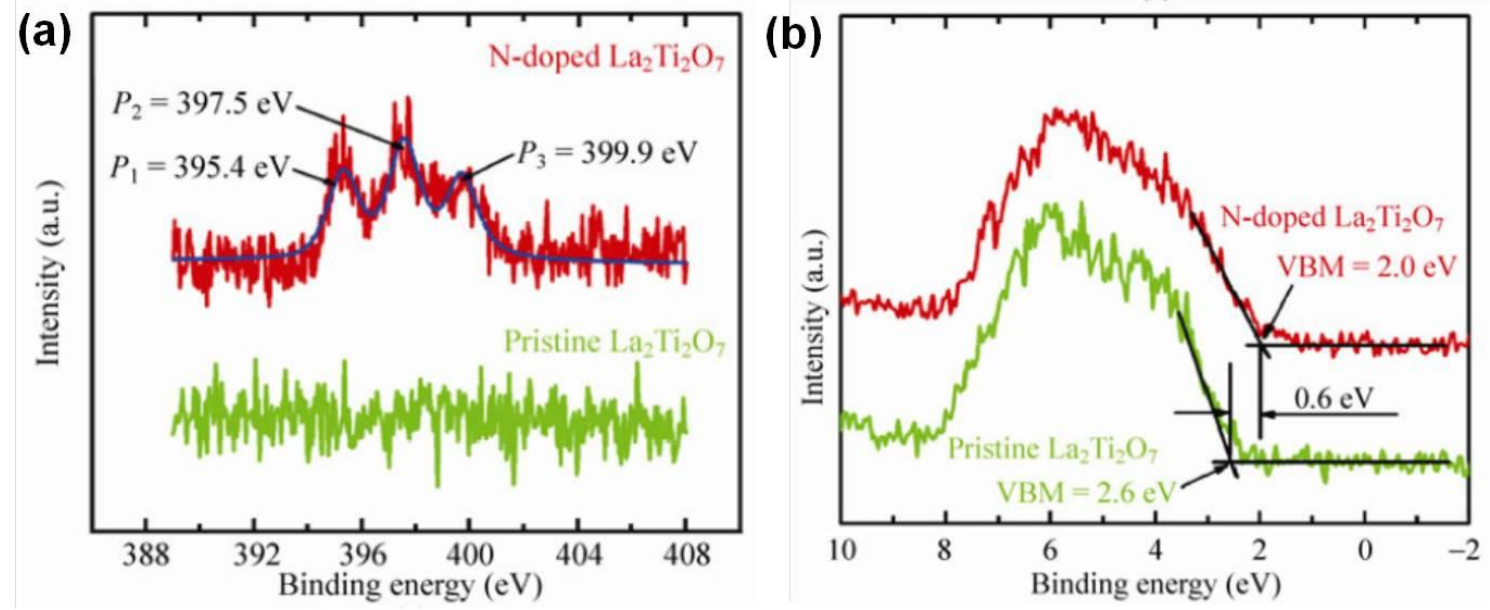

Figure 4-5 XPS spectra obtained from the pristine and the nitrogendoped $\mathrm{La}_{2} \mathrm{Ti}_{2} \mathrm{O}_{7}$ nanosheets showing the $\mathrm{N}$ 1s core level (a), and the valence band (b).

No nitrogen was detected in the pristine $\mathrm{La}_{2} \mathrm{Ti}_{2} \mathrm{O}_{7}$ nanosheets by XPS (Figure 4-5 (a)). XPS analysis showed that the nanosheets contained 3.3 at. \% of nitrogen after $\mathrm{NH}_{3}$ treatment. The N 1s core-level XPS spectrum in Figure 4-5 (a), which was obtained from the $\mathrm{N}$-doped nanosheets, displayed three distinct peaks. The $\mathrm{N} 1 \mathrm{~s}$ peak at $399.9 \mathrm{eV}$ can be assigned to the $\mathrm{Ti}-(\mathrm{N}-\mathrm{O})$ bond [27]. The $\mathrm{N} 1 \mathrm{~s}$ peak at $395.4 \mathrm{eV}$ corresponds to the $\mathrm{Ti}-\mathrm{N}$ bond, indicating that some doped nitrogen atoms were directly bonded to the titanium atoms in the $\mathrm{TiO} 6$ octahedron [27-29]. An additional peak at $397.5 \mathrm{eV}$, located between the above two peaks, is generally ascribed to the $\mathrm{N}-\mathrm{Ti}-\mathrm{O}$ bond [30]. 

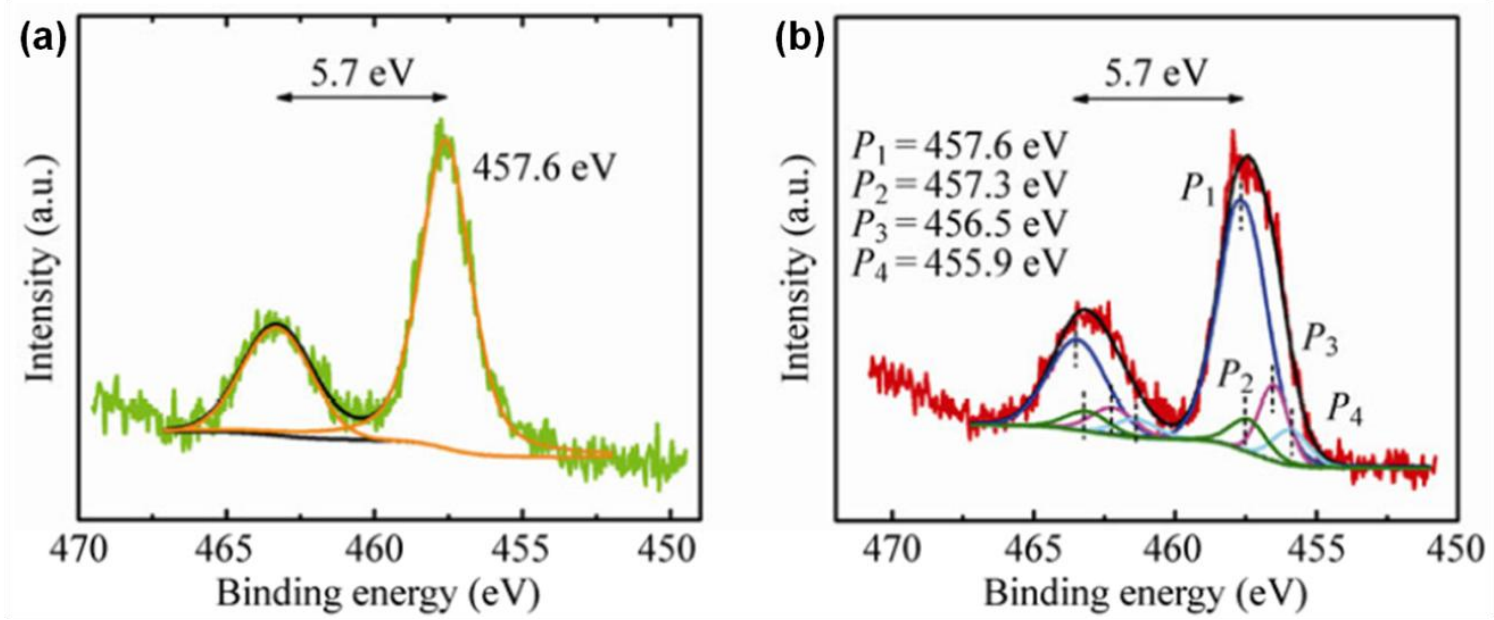

Figure 4-6 XPS spectra of the Ti 2p core-level of the pristine (a) and the nitrogen-doped (b) $\mathrm{La}_{2} \mathrm{Ti}_{2} \mathrm{O}_{7}$ nanosheets.

The assignment of the $\mathrm{N} 1 \mathrm{~s}$ peaks was confirmed by the difference in the Ti $2 \mathrm{p}$ peaks before and after nitrogen-doping (Figure 4-6). The Ti 2p core level spectrum of the pristine $\mathrm{La}_{2} \mathrm{Ti}_{2} \mathrm{O}_{7}$ nanosheets exhibited a symmetric $\mathrm{Ti} 2 \mathrm{p} 3 / 2$ peak at $457.6 \mathrm{eV}$, which can be assigned to the $\mathrm{Ti}-\mathrm{O}$ bond. In contrast, the $\mathrm{Ti} 2 \mathrm{p} 3 / 2$ peak of the $\mathrm{N}$-doped nanosheets was asymmetric and can be deconvoluted into four components. Because only 3.3 at. \% of nitrogen was doped into the $\mathrm{La}_{2} \mathrm{Ti}_{2} \mathrm{O}_{7}$ lattice, most of $\mathrm{TiO}_{6}$ octahedron slabs did not contain nitrogen atoms. Therefore, the major component can still be assigned to the Ti-O bonds. The component at $457.3 \mathrm{eV}$ can be attributed to the $\mathrm{Ti}-(\mathrm{N}-\mathrm{O})$ bond. The peak at $456.5 \mathrm{eV}$ corresponds to the $\mathrm{N}-\mathrm{Ti}-\mathrm{O}$ bond. The fourth component at $455.9 \mathrm{eV}$ can be ascribed to the Ti-N bond, on the basis of the reference literature [28]. Therefore, it is clear that nitrogen atoms have been successfully doped into the $\mathrm{La}_{2} \mathrm{Ti}_{2} \mathrm{O}_{7}$ lattice. 


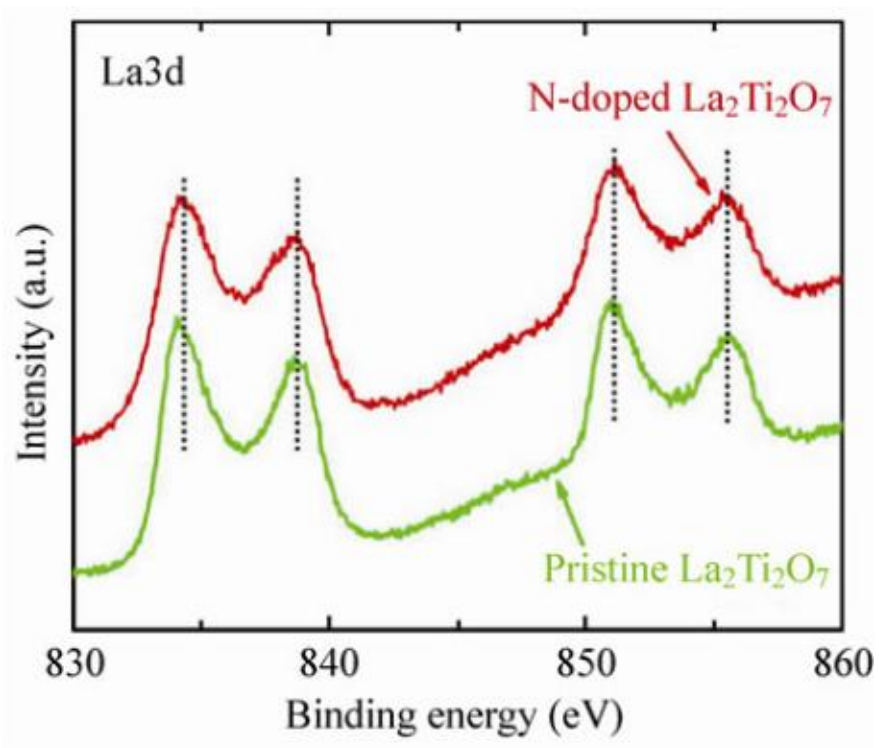

Figure 4-7 XPS spectra of the $\mathrm{La} 3 \mathrm{~d}$ core-level of the pristine and nitrogen-doped $\mathrm{La}_{2} \mathrm{Ti}_{2} \mathrm{O}_{7}$

It is interesting that there was no change in the La $3 \mathrm{~d}$ doublet peak before and after N-doping in Figure 4-7. This strongly suggests that the doped nitrogen atoms are preferentially positioned in the $\mathrm{TiO}_{6}$ octahedron slabs, in agreement with previous studies $[15,31]$. Figure 4-5 (b) shows the valence band spectra of the pristine and the nitrogendoped $\mathrm{La}_{2} \mathrm{Ti}_{2} \mathrm{O}_{7}$ nanosheets. The valence band maximum (VBM) of the nanosheets exhibited a red shift of $0.60 \mathrm{eV}$ after nitrogen doping. 


\subsubsection{Light absorption}

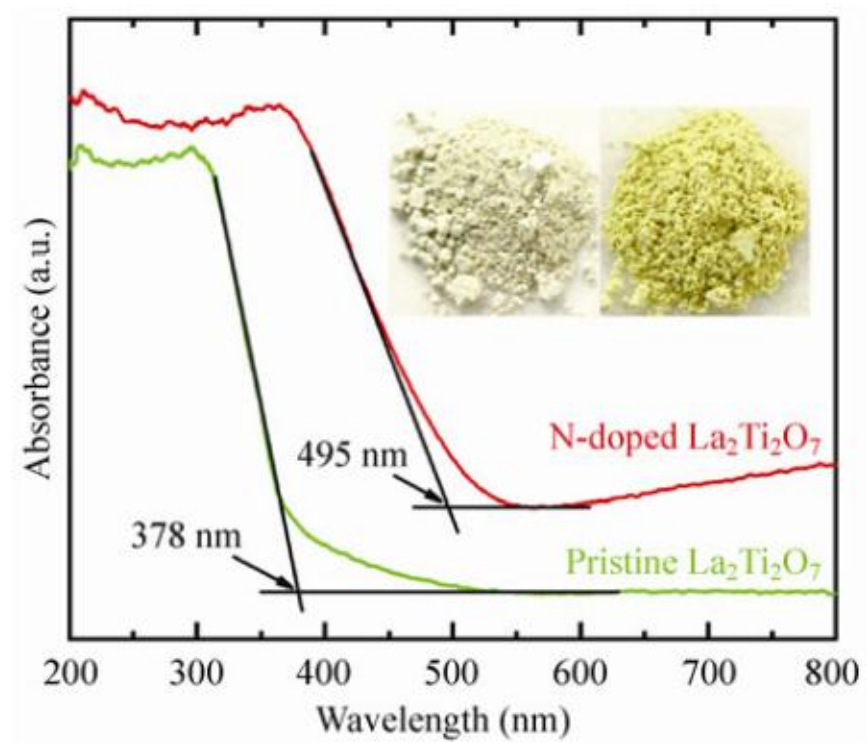

Figure 4-8 Diffuse reflectance spectra of the pristine and nitrogendoped $\mathrm{La}_{2} \mathrm{Ti}_{2} \mathrm{O} 7$ nanosheets. The insets are the optical photos of the nanosheet samples, showing the change in the sample color from white (left) to yellow (right) due to nitrogen-doping.

The color change of the $\mathrm{La}_{2} \mathrm{Ti}_{2} \mathrm{O}_{7}$ nanosheets from white to yellow after nitrogen doping was visible to the naked eye (as shown in the inset in Figure 4-8). The light absorption property of the nanosheets was further evaluated by UV-visible spectroscopy (Figure 4-8). The optical absorption edge of the pristine $\mathrm{La}_{2} \mathrm{Ti}_{2} \mathrm{O}_{7}$ nanosheets, had a cut off at $378 \mathrm{~nm}$, which corresponds to an optical band gap of $3.28 \mathrm{eV}$. The optical absorption edge of the $\mathrm{N}$-doped nanosheets was extended to $495 \mathrm{~nm}$, which is equivalent to an optical band gap of $2.51 \mathrm{eV}$. This indicates that nitrogen doping resulted in band gap shift of $0.77 \mathrm{eV}$. 

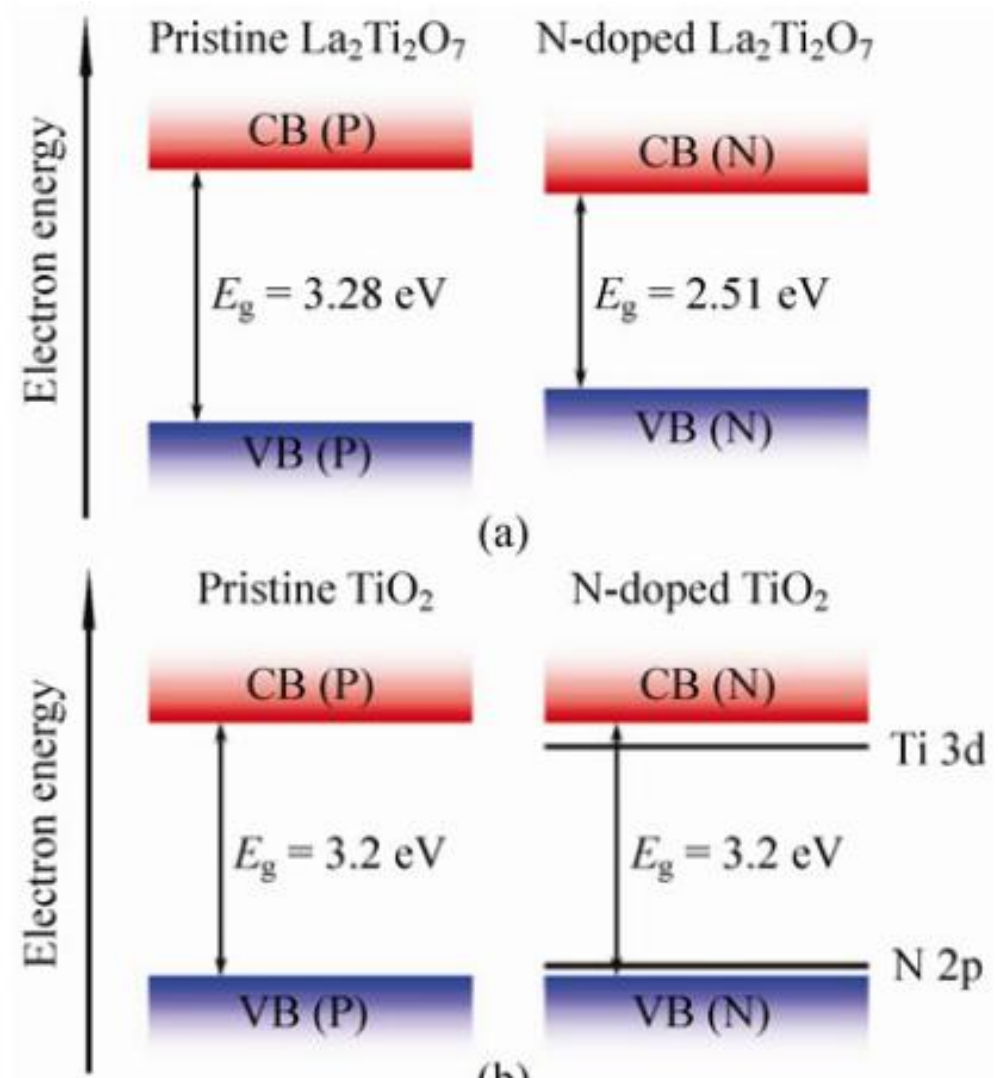

(b)

Figure 4-9 Schematic illustration of the effects of nitrogen-doping on the band structures of pristine $\mathrm{La}_{2} \mathrm{Ti}_{2} \mathrm{O}_{7}(\mathrm{a})$, and anatase $\mathrm{TiO}_{2}(\mathrm{~b})$.

It is worth noting that nitrogen-doping into $\mathrm{La}_{2} \mathrm{Ti}_{2} \mathrm{O}_{7}$ nanosheets induced a parallel shift of the whole optical absorption edge to the longer wavelength direction. This behavior is totally different from that of the $\mathrm{N}$-doped anatase $\mathrm{TiO}_{2}$ samples. Nitrogen-doping into $\mathrm{TiO}_{2}$ imposes an add-on shoulder on the cutoff edge of the absorption spectrum [10], which is due to the fact that nitrogen-doping results in the localized N 2p states near the valence band edge, as schematically illustrated in Figure 49 (b). However, the parallel shift of the whole optical absorption edge indicates that the valence band was broadened after the $\mathrm{La}_{2} \mathrm{Ti}_{2} \mathrm{O}_{7}$ nanosheets were doped with nitrogen, 
leading to the upshift of the valence band edge in Figure 4-9 (a). In other words, nitrogendoping into $\mathrm{TiO}_{2}$ induces localized states above the valence band edge while nitrogendoping into the $\mathrm{La}_{2} \mathrm{Ti}_{2} \mathrm{O}_{7}$ nanosheets results in the narrowing of the band gap (Figure 4-9). This conclusion was supported by the XPS spectra of the valence band shown in Figure $4-5$, in which the valence band maximum showed a red shift after nitrogen doping. The shift-up of the valence band edge could be due to the fact that that the $\mathrm{N} 2 \mathrm{p}$ orbitals of the dopant become hybridized with the $\mathrm{O} 2 \mathrm{p}$ orbital of the valence band of perovskite. Indeed, previous density functional theory (DFT) calculations showed that the $\mathrm{N} 2 \mathrm{p}$ orbital of the dopant was hybridized with the $\mathrm{O} 2 \mathrm{p}$ orbital of the valence band of perovskite to form $\mathrm{AO}_{x} \mathrm{~N}_{y}(\mathrm{~A}=$ metal cation, $x+y=6)$ octahedral structure slabs in the $\mathrm{La}_{2} \mathrm{Ti}_{2} \mathrm{O}_{7}$ sample after treatment in $\mathrm{NH}_{3}[15]$.

\subsubsection{Photocatalytic activity}
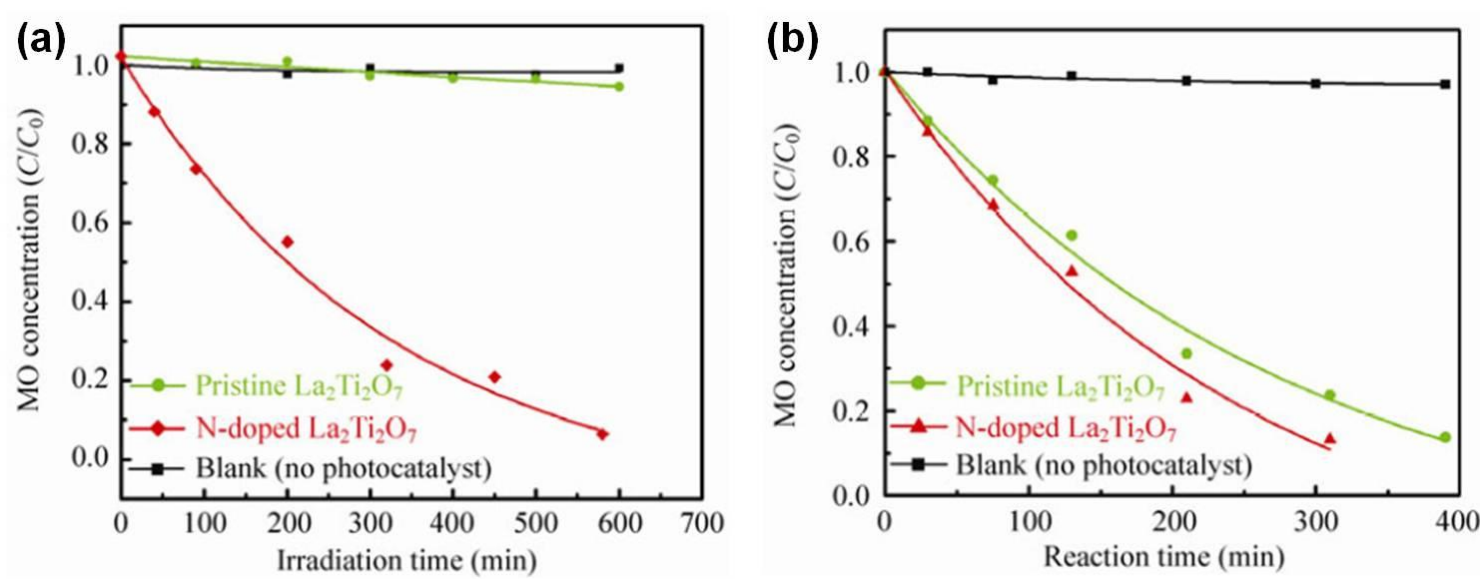

Figure 4-10 Photocatalytic activities of the pristine and the nitrogendoped $\mathrm{La}_{2} \mathrm{Ti}_{2} \mathrm{O} 7$ nanosheets under visible light irradiation (a), and under ultraviolet light irradiation (b). 
The photocatalytic activities of the nanosheets were evaluated by monitoring the decomposition of methyl orange in aqueous solution under visible and UV light irradiation. Figure 4-10 (a) shows the degradation curves of methyl orange induced by the pristine and the $\mathrm{N}$-doped $\mathrm{La}_{2} \mathrm{Ti}_{2} \mathrm{O}_{7}$ nanosheets under visible light irradiation. As a control experiment, the blank curve represents the change in the concentration of methyl orange in aqueous solution in the absence of any photocatalyst. After $10 \mathrm{~h}$ of visible light irradiation, the decomposition of methyl orange in the absence of a photocatalyst was less than $3 \%$, which was within the experimental tolerance range. Addition of the pristine $\mathrm{La}_{2} \mathrm{Ti}_{2} \mathrm{O}_{7}$ nanosheets into the solution did not accelerate the decomposition of methyl orange. In other words, no photocatalytic activity was observed for the pristine $\mathrm{La}_{2} \mathrm{Ti}_{2} \mathrm{O}_{7}$ nanosheets. In contrast, methyl orange was completely decomposed in the presence of the nitrogen-doped $\mathrm{La}_{2} \mathrm{Ti}_{2} \mathrm{O}_{7}$ nanosheets after $10 \mathrm{~h}$ of visible light irradiation. This clearly shows that the $\mathrm{N}$-doped $\mathrm{La}_{2} \mathrm{Ti}_{2} \mathrm{O}_{7}$ had an excellent visible light photocatalytic activity. In addition, the photocatalytic activity of the nitrogen-doped $\mathrm{La}_{2} \mathrm{Ti}_{2} \mathrm{O}_{7}$ nanosheets showed better photocatalytic activity than that of the pristine $\mathrm{La}_{2} \mathrm{Ti}_{2} \mathrm{O}_{7}$ nanosheets under UV light irradiation (Figure 4-10 (b)). As described in the previous sections, the shape and the dimensions of the $\mathrm{La}_{2} \mathrm{Ti}_{2} \mathrm{O}_{7}$ nanosheets did not change after nitrogen doping. In addition, no phase transition occurred during $\mathrm{NH}_{3}$ treatment. The enhancement of photocatalytic activity can therefore be attributed to the narrowing of the band gap of $\mathrm{La}_{2} \mathrm{Ti}_{2} \mathrm{O}_{7}$ nanosheets.

This work demonstrates that the nitrogen-doped $\mathrm{La}_{2} \mathrm{Ti}_{2} \mathrm{O}_{7}$ nanosheets not only showed significant visible light photocatalytic activity but also enhanced the UV light photocatalytic activity. In contrast, our previous studies revealed that nitrogen-doping 
into anatase $\mathrm{TiO}_{2}$ led to limited visible light photocatalytic activity but did not improve UV light photocatalytic activity at a low levels of nitrogen-doping and even had an adverse effect on the UV light photocatalytic activity at high levels of nitrogen-doping [10]. The difference in the photocatalytic performance of the $\mathrm{N}$-doped $\mathrm{La}_{2} \mathrm{Ti}_{2} \mathrm{O}_{7}$ nanosheets and the $\mathrm{N}$-doped anatase $\mathrm{TiO}_{2}$ can be ascribed to the different changes in the band structure induced by nitrogen-doping. Nitrogen-doping results in the narrowing of the band gap of $\mathrm{La}_{2} \mathrm{Ti}_{2} \mathrm{O}_{7}$ nanosheets (Figure 4-9 (a)) while it induces localized mid-gap states in the band structure of anatase $\mathrm{TiO}_{2}$ (Figure 4-9 (b)). The difference in the band structure results in differences in light absorption, the mobility of charger carriers and the recombination rate of photogenerated electron-hole pairs.

For the $\mathrm{N}$-doped $\mathrm{La}_{2} \mathrm{Ti}_{2} \mathrm{O}_{7}$ nanosheets under visible light irradiation, electrons are excited to the conduction band at lowered energy from the broadened valence band, generating holes in the valence band. The photogenerated electrons and holes are delocalized and have high mobility in the conduction band and the valence band, respectively. For the $\mathrm{N}$-doped anatase $\mathrm{TiO}_{2}$ under visible light irradiation, electrons cannot jump to the conduction band from the valence band due to the large band gap. Electrons jump to the conduction band only from the localized $N 2 p$ midgap state near the valence band, creating holes in the $\mathrm{N} 2 \mathrm{p}$ state, as shown in Fig. 4(b). The photogenerated holes in the localized N 2p state have very low mobility [6]. Therefore, nitrogen-doping significantly enhances the visible light photocatalytic activity of $\mathrm{La}_{2} \mathrm{Ti}_{2} \mathrm{O}$, while bringing limited visible light photocatalytic activity to $\mathrm{TiO}_{2}$.

$\mathrm{N}$-doped $\mathrm{La}_{2} \mathrm{Ti}_{2} \mathrm{O}_{7}$ has a narrower band gap than the pristine $\mathrm{La}_{2} \mathrm{Ti}_{2} \mathrm{O}_{7}$ (Figure 4-9 (a)). Hence more electrons and holes are generated in $\mathrm{N}$-doped $\mathrm{La}_{2} \mathrm{Ti}_{2} \mathrm{O}_{7}$ than in pristine 
$\mathrm{La}_{2} \mathrm{Ti}_{2} \mathrm{O}_{7}$ under UV light irradiation. Narrowing of the band gap has no adverse effect on the photocatalyst activity. As a result, the $\mathrm{N}$-doped $\mathrm{La}_{2} \mathrm{Ti}_{2} \mathrm{O}_{7}$ nanosheets exhibited much better UV light photocatalytic activity than the pristine $\mathrm{La}_{2} \mathrm{Ti}_{2} \mathrm{O}_{7}$ nanosheets. For Ndoped $\mathrm{TiO}_{2}$ under UV light irradiation, besides the photogenerated charge carriers in the valence band and the conduction band, some additional charge carriers are also produced in the localized states in the mid-gap although the number is limited, which is beneficial to the photocatalytic activity. However, the localized states in the mid-gap also act as sites for recombination of the photogenerated charge carriers, which has an adverse effect on the photocatalytic activity. This adverse effect cancels out or even overrides the positive effect. Therefore, nitrogen-doping is not able to improve the UV light photocatalytic activity of $\mathrm{TiO}_{2}$, and may even make it worse.

\subsection{Conclusions}

Perovskite-type layer-structured $\mathrm{La}_{2} \mathrm{Ti}_{2} \mathrm{O}_{7}$ nanosheets were successfully prepared by a hydrothermal method. Heat treatment in $\mathrm{NH}_{3}$ flow resulted in the incorporation of the nitrogen atoms into the perovskite $\mathrm{La}_{2} \mathrm{Ti}_{2} \mathrm{O}_{7}$ lattice. The $\mathrm{N}$-doped $\mathrm{La}_{2} \mathrm{Ti}_{2} \mathrm{O}_{7}$ nanosheets exhibited a single-crystalline structure with a thickness of $\sim 15 \mathrm{~nm}$. Nitrogen-doping leads to a narrowing of the band gap of the $\mathrm{La}_{2} \mathrm{Ti}_{2} \mathrm{O}_{7}$ nanosheets by $0.77 \mathrm{eV}$ but no change in the particle shape, the dimensions or the crystal phase. The pristine $\mathrm{La}_{2} \mathrm{Ti}_{2} \mathrm{O}_{7}$ nanosheets showed no photocatalytic activity under visible light irradiation. In contrast, the nitrogendoped $\mathrm{La}_{2} \mathrm{Ti}_{2} \mathrm{O}_{7}$ nanosheets not only showed significant visible light photocatalytic activity but also enhanced UV light photocatalytic activity. The enhancement of photocatalytic activity originates from the narrowing of the band gap of the $\mathrm{La}_{2} \mathrm{Ti}_{2} \mathrm{O}_{7}$ 
nanosheets. The present work has important implications for the development of new visible light photocatalysts.

\section{Reference}

[1] Fujishima, A.; Honda, K. Nature 1972, 238, 37.

[2] Linsebigler, A. L.; Lu, G. Q.; Yates, J. T. Chem. Rev. 1995, 95, 735.

[3] Hashimoto, K.; Irie, H.; Fujishima, A. Jpn. J. Appl. Phys. 2005, 44, 8269.

[4] Kudo, A.; Miseki, Y. Chem. Soc. Rev. 2009, 38, 253.

[5] He, Z. Q.; Xu, X.; Song, S.; Xie, L.; Tu, J. J.; Chen, J. M.; Yan, B. A. J. Phys. Chem. C 2008, 112, 16431.

[6] Tafen, D. N.; Wang, J.; Wu, N. Q.; Lewis, J. P. Appl. Phys. Lett. 2009, 94, 093101.

[7] Sakthivel, S.; Kisch, H. Angew. Chem. Int. Ed. 2003, 42,4908.

[8] Yamashita, H.; Harada, M.; Misaka, J.; Takeuchi, M.; Ichihashi, Y.; Goto, F.; Ishida, M.; Sasaki, T.; Anpo, M. J. Synchrot. Radiat. 2001, 8. 569.

[9] Henderson, M. A.; White, J. M.; Uetsuka, H.; Onishi, H. J. Am. Chem. Soc. 2003, 125, 14974.

[10] Wang, J.; Tafen, D. N.; Lewis, J. P.; Hong, Z. L.; Manivannan, A.; Zhi, M. J.; Li, M.; Wu, N. Q. J. Am. Chem. Soc. 2009, 131, 12290.

[11] Jeong, H.; Kim, T.; Kim, D.; Kim, K. Int. J. Hydrogen Energy, 2006, 31, 1142-1146. [12] Takata, T.; Furumi, Y.; Shinohara, K.; Tanaka, A.; Hara, M.; Kondo, J. N.; Domen, K. Chem.Mater. 1997, 9, 1063-1064.

[13] Kim, H. G.; Hwang, D. W.; Kim, J.; Kim, Y. G.; Lee, J. S. Chem.Commun. 1999, 1077. 
[14] Abe, R.; Higashi, M.; Sayama, K.; Abe, Y.; Sugihara, H. J. Phys. Chem. B 2006, 110, 2219.

[15] Kasahara, A.; Nukumizu, K.; Takata, T.; Kondo, J. N.; Hara, M.; Kobayashi, H.; Domen, K. J. Phys. Chem. B 2003, 107, 791-797.

[16] Song, H. B.; Peng, T. Y.; Cai, P.; Yi, H. B.; Yan, C. H. Catal. Lett. 2007, 113, 54-58.

[17] Li, Z. H.; Xue, H.; Wang, X. X.; Fu, X. Z. J. Mol. Catal. A-Chem. 2006, 260, 56-61.

[18] Hwang, D. W.; Kim, H. G.; Lee, J. S.; Kim, J.; Li, W.; Oh, S. H. J. Phys. Chem. B 2005, 109, 2093-2102.

[19] Kudo, A.; Niishiro, R.; Iwase, A.; Kato, H. Chem. Phys. 2007, 339, 104-110.

[20] Hwang, D. W.; Cha, K. Y.; Kim, J.; Kim, H. G.; Bae, S. W.; Lee, J. S. Ind. Eng. Chem. Res. 2003, 42, 1184-1189.

[21] Kim, J.; Hwang, D. W.; Kim, H. G.; Bae, S. W.; Ji, S. M.; Lee, J. S. Chem. Commun. 2002, 2488-2489.

[22] Luo, W. J.; Li, Z. S.; Jiang, X. J.; Yu, T.; Liu, L. F.; Chen, X. Y.; Ye, J. H.; Zou, Z. G.

Phys. Chem. Chem. Phys. 2008, 10, 6718-6723.

[23] Kim, J.; Hwang, D. W.; Kim, H. G.; Bae, S. W.; Lee, J. S.; Li, W.; Oh, S. H. Top. Catal. 2005, 35, 295-303.

[24] Li, K. W.; Wang, Y.; Wang, H.; Zhu M. K.; Yan, H. Nanotechnology 2006, 17, $4863-4867$.

[25] Wu, N. Q.; Wang, J.; Tafen, D. N.; Wang, H.; Zheng, J. G.; Lewis, J. P.; Liu, X. G.; Leonard, S. S.; Manivannan, A. J. Am. Chem. Soc. 2010, 132, 6679-6685. 
[26] Kim, H. G.; Hwang, D. W.; Bae, S. W.; Jung, J. H.; Lee, J. S. Catal. Lett. 2003, 91, 193.

[27] Fujishima, A.; Zhang, X. T.; Tryk, D. A. Surf. Sci. Rep. 2008, 63, 515-582.

[28] Moulder, J. F.; Stickle, W. F.; Sobol, P. E.; Bomben, K. D. Handbook of X-ray Photoelectron Spectroscopy; Physical Electronics: Eden Prairie, 1995.

[29] Esaka, F.; Furuya, K.; Shimada, H.; Imamura, M.; Matsubayashi, N.; Sato, H.; Nishijima, A.; Kawana, A.; Ichimura, H.; Kikuchi, T. J. Vac. Sci. Technol. A 1997, 15, $2521-2528$.

[30] Kasahara, A.; Nukumizu, K.; Hitoki, G.; Takata, T.; Kondo, J. N.; Hara, M.; Kobayashi, H.; Domen, K. J. Phys. Chem. A 2002, 106, 6750-6753.

[31] Sathish, M.; Viswanathan, B.; Viswanath, R. P.; Gopinath, C. S. Chem. Mater. 2005, 17,6349 . 


\section{Chapter 5 Photocatalytic Generation of Hydrogen with Visible- light Nitrogen-doped Lanthanum Titanium Oxides}

\subsection{Background and introduction}

As we discussed in the introduction, the energy shortage and environmental disruption become a global concern, there is an incentive to produce clean fuels in a sustainable fashion to replace fossil fuels. Solar fuel production recently attracted increasing attention. Hydrogen has been generated by directly splitting water with photocatalysts under the solar light irradiation [1-3]. $\mathrm{TiO}_{2}$ is the most common photocatalyst used for solar hydrogen generation due to its stability, low cost and environmental benignancy [4-6]. The perovskite semiconductor photocatalyst family including $\mathrm{La}_{2} \mathrm{Ti}_{2} \mathrm{O}_{7}$ (LTO), $\mathrm{Sr}_{3} \mathrm{Ti}_{2} \mathrm{O}_{7}$ (STO) and $\mathrm{K}_{2} \mathrm{La}_{2} \mathrm{Ti}_{3} \mathrm{O}_{10}$ (KLTO) were found to be alternative photocatalysts for water splitting due to their unique perovskite layered structure [7-10]. It has been reported that the periodically ranged corner-shared $\mathrm{TiO}_{6}$ octahedrons and La cations in the LTO phase can boost the mobility of charge carriers [3]. In Chapter 4 we showed the photocatalytic activity of LTO could be improved in the UV and visible by the introduction of a continuum of doped states near the valence band edge. Therefore, in this chapter we extend the study from methyl orange degradation into hydrogen production to see if the same enhancements, and the results regarding the harmful effects of mid gap states, extend to a practical application.

As introduced in Chapter 4, there are two major hurdles in the development of LTO photocatalyst for water-splitting. First, the LTO synthesis is currently performed via 
a solid-state reaction (SSR). SSR requires high reaction temperature typically above $1100{ }^{\circ} \mathrm{C}[9,11]$, which results in large LTO particles on the micrometer scale, leading to a very low catalytic surface area $[3,12]$. Second, similar to $\mathrm{TiO}_{2}$, the wide band gap $(3.3-$ $3.9 \mathrm{eV}$ ) of LTO limits its light harvesting only in the ultraviolet (UV) light range. The UV light comprises less than $5 \%$ of natural solar light radiation. Many attempts have been made to improve the photocatalytic performance of LTO. For example, ion doping could effectively enhance the visible light absorption of LTO. However, doping at hightemperature SSR decreases the surface area of the photocatalysts $[11,13]$. Furthermore, cation doping only introduces the mid-gap states into the band gap, and cannot enhance the visible-light-responsive photocatalytic activity effectively even though it enhances the light absorption $[3,11]$. In order to obtain large specific surface area, LTO was prepared at a lower reaction temperature $[14,15]$. Unfortunately, the band gap of pristine LTO obtained was still large. Hence the photocatalytic hydrogen generation from water splitting was conducted only in the UV light range [2].

It is highly desirable to develop a synthesis approach that extends the photocatalysis into the visible light range while creating a high specific surface area. Here the hydrothermal process is employed to fabricate single-crystalline perovskite LTO nanosheet photocatalysts at a low temperature. The as-synthesized LTO has showed twodimensional nanosheet morphology. Our previous report has shown that one-dimensional (1-D) and 2-D nanostructures exhibit higher mobility and lower recombination rate of charge carriers than zero-dimensional (0-D) nanoparticles $[6,16]$. Therefore, $1-\mathrm{D}$ and 2-D nanostructures show better photocatalytic activity than 0 -D counterparts. In addition, the nanosheets are doped with nitrogen to increase the visible light absorption. As a result, 
the N-doped LTO nanosheet exhibit good photocatalytic activity toward hydrogen generation.

\subsection{Experimental section}

\subsubsection{Chemicals and materials}

Tetraammineplatinum(II) chloride hydrate $\left(\mathrm{Pt}\left(\mathrm{NH}_{3}\right) 4 \mathrm{Cl}_{2} \cdot \mathrm{xH}_{2} \mathrm{O}, \geq 98.0 \%\right.$, powder), lanthanum(III) nitrate hexahydrate $\left(\mathrm{La}\left(\mathrm{NO}_{3}\right)_{3} \cdot 6 \mathrm{H}_{2} \mathrm{O}, \geq 99.0 \%\right.$, particles $)$, sodium hydroxide ( $\mathrm{NaOH}, \geq 98 \%$, pellets) were purchased from Sigma-Aldrich. Titanium sulfate nonahydrate $\left(\mathrm{Ti}\left(\mathrm{SO}_{4}\right)_{2} \cdot 9 \mathrm{H}_{2} \mathrm{O}\right)$ was purchased from Chem Service, Inc. The resistivity of deionized water was $18.2 \mathrm{M} \cdot \mathrm{cm}$ at $25^{\circ} \mathrm{C}$.

\subsubsection{Photocatalyst synthesis}

$0.5 \mathrm{~g}$ of $\mathrm{Ti}\left(\mathrm{SO}_{4}\right)_{2} \cdot 9 \mathrm{H}_{2} \mathrm{O}$ and $0.9 \mathrm{~g}$ of $\mathrm{La}\left(\mathrm{NO}_{3}\right)_{3} \cdot 6 \mathrm{H}_{2} \mathrm{O}$ were dissolved into $20 \mathrm{~mL}$ of $1.0 \mathrm{M} \mathrm{NaOH}$ aqueous solution. The mixed solution was stirred in ultrasonic bath vigorously for at least $30 \mathrm{~min}$ and then sealed into a $30 \mathrm{~mL}$ of Teflon-lined autoclave. The autoclave was placed into a pre-heated oven at $240{ }^{\circ} \mathrm{C}$ for $24 \mathrm{~h}$ for hydrothermal processing. After hydrothermal treatment, the autoclave was cooled down to room temperature in air. As a result, whitish fluffy powder was obtained. The collected powder was rinsed with deionized water and centrifuged to reduce the $\mathrm{pH}$ of the solution close to 7. The product was dried in air at $80{ }^{\circ} \mathrm{C}$ overnight, and then heated to $700{ }^{\circ} \mathrm{C}$ for $1 \mathrm{~h}$ to obtain the pristine LTO nanosheets (P-LTO). 
The surface of the LTO nanosheets was further loaded with the platinum nanoparticles by impregnation. Briefly, $0.5 \mathrm{~g}$ of the LTO powder and $0.05 \mathrm{~g}$ of $\mathrm{Pt}\left(\mathrm{NH}_{3}\right)_{4} \mathrm{Cl}_{2}$ powder were added into $20 \mathrm{~mL}$ of deionized water to form a suspension. The suspension was then stirred for $24 \mathrm{~h}$. The suspension was centrifuged at a high speed for $2 \mathrm{~h}$ to obtain the white powder. The powder was dried at $80{ }^{\circ} \mathrm{C}$ overnight and then heated at $300{ }^{\circ} \mathrm{C}$ in air for $2 \mathrm{~h}$. The powder was then heated in the $\mathrm{H}_{2}$ flow at a flow rate of 20 $\mathrm{cm}^{3} / \mathrm{min}$ at $500{ }^{\circ} \mathrm{C}$ for $1 \mathrm{~h}$. Consequently, the Pt-loaded pristine LTO nanosheets (Pt@PLTO) were obtained.

Finally, nitrogen doping was performed on the Pt@P-LTO to obtain the Pt-loaded nitrogen-doped LTO nanosheets (Pt@N-LTO). The detail procedure for nitrogen doping was described previously [7]. Additionally, the nitrogen-doped LTO nanosheets (N-LTO) without Pt loading were synthesized by doping nitrogen directly into the LTO nanosheets.

\subsubsection{Photocatalyst characterization}

The morphology of the photocatalysts was observed with a field emission scanning electron microscope (FE-SEM) (JEOL 7600F), a transmission electron microscope (TEM) and a high-resolution TEM (HRTEM, JEOL JEM 2100F) equipped with energy-dispersive X-ray spectroscopy (EDX). The crystal structure of the samples were analyzed by X-ray Diffraction (XRD, Bruker D8 Discovery X-ray Diffractometer)The chemical status of N and Pt in the LTO nanosheets was analyzed by X-ray photoelectron spectroscopy (XPS) (PHI 5000 Versa Probe, Physical Electronics, $\mathrm{MN})$. The $\mathrm{C} 1 \mathrm{~s}$ peak at $284.8 \mathrm{eV}$ was used as the reference for calibrating the XPS spectra. The light-absorption properties were characterized by the diffuse-reflection mode 
with Shimadzu 2550 UV-Visible spectrometer equipped with an integrating sphere (UV 2401/2, Shimadzu). Prior to measurement, the sample powder was pressed onto a $\mathrm{BaSO}_{4}$ plate to form a pellet. The specific surface area of the pristine LTO nanosheets was determined using the Brunauer-Emmett-Teller (BET) method.

\subsubsection{Hydrogen generation by photocatalytic water splitting}

A commercial photoreactor (LUZ-4V, Luzchem) equipped with fourteen $8 \mathrm{~W}$ of visible (Luzchem LZC-420, centered at $420 \mathrm{~nm}$ ) or ultraviolet (Luzchem LZC-UVA, centered at $360 \mathrm{~nm}$ ) light lamps were used as the visible light and the UV light sources, respectively. The light intensity was 50-60 mW/cm². Four samples (Pt@NLTO, Pt@PLTO, N-LTO and P-LTO) were used for photocatalytic hydrogen generation, respectively. Water splitting was carried out according to the following procedure. $0.2 \mathrm{~g}$ of the photocatalyst sample was suspended into $10 \mathrm{~mL}$ of deionized water and stirred for $30 \mathrm{~s}$. The solution was then transferred into four quartz flasks, followed by injection of high purity $\mathrm{N}_{2}$, which was sealed with a septum flask stopper. The samples were irradiated with UV or visible light for $5,10,15$ and $20 \mathrm{~h}$, respectively. During the photocatalytic reaction, the solution was always stirred magnetically. Finally, $5 \mathrm{~mL}$ of gas generated was extracted from each flask to analyze the amount of $\mathrm{H}_{2}$ produced at each reaction time with a gas chromatography (GC) equipped with a thermal conductivity detector (TCD). 


\subsection{Results and discussion}

\subsubsection{Microstructure of photocatalysts}
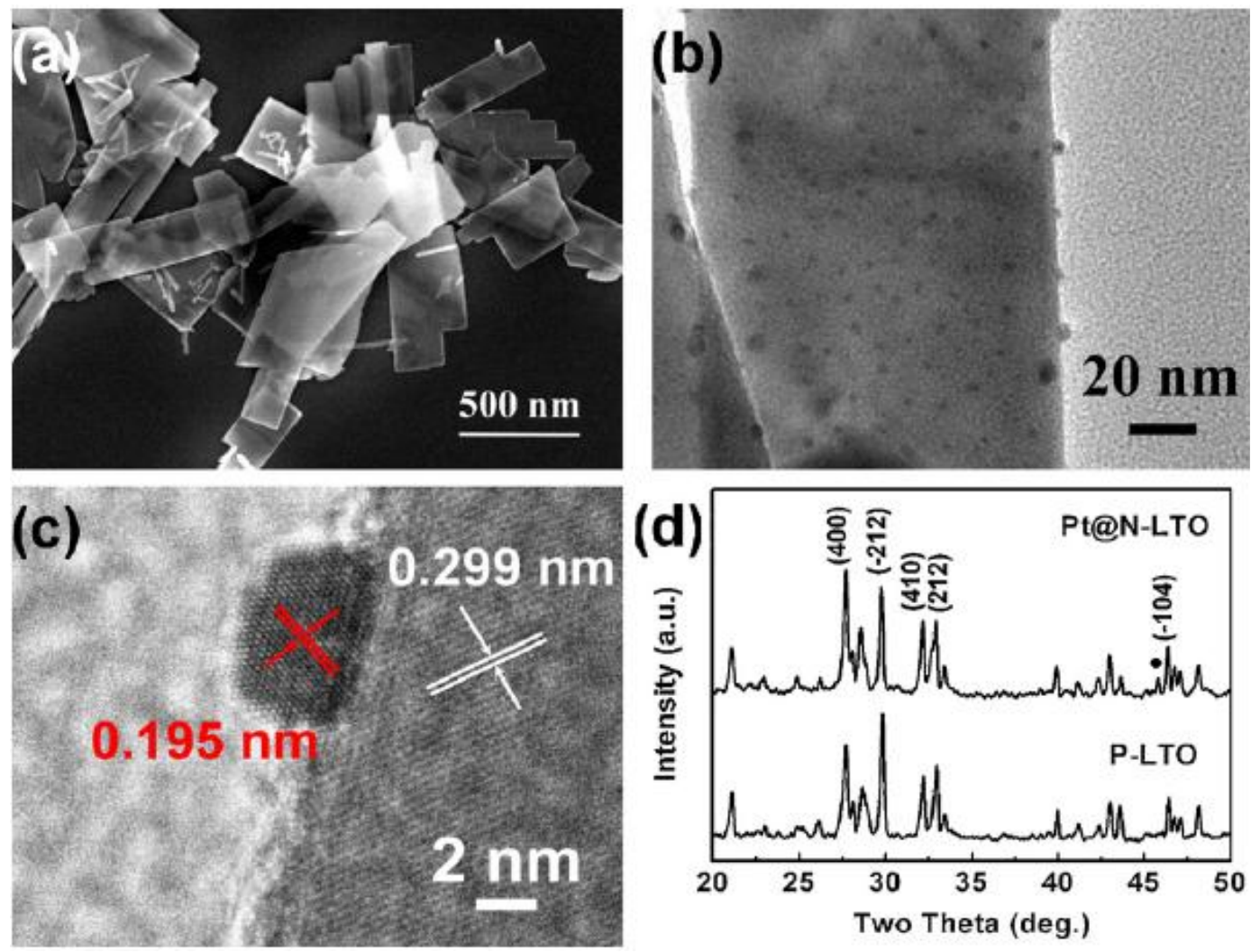

Figure 5-1 (a) SEM image of the pristine $\mathrm{La}_{2} \mathrm{Ti}_{2} \mathrm{O}_{7}$ nanosheets; (b) TEM image of the Pt-loaded nitrogendoped $\mathrm{La}_{2} \mathrm{Ti}_{2} \mathrm{O}_{7}$ nanosheets; (c) HRTEM image of the Pt-loaded nitrogendoped nanosheets and (d) XRD patterns of the pristine and the Pt-loaded nitrogen-doped nanosheets. 


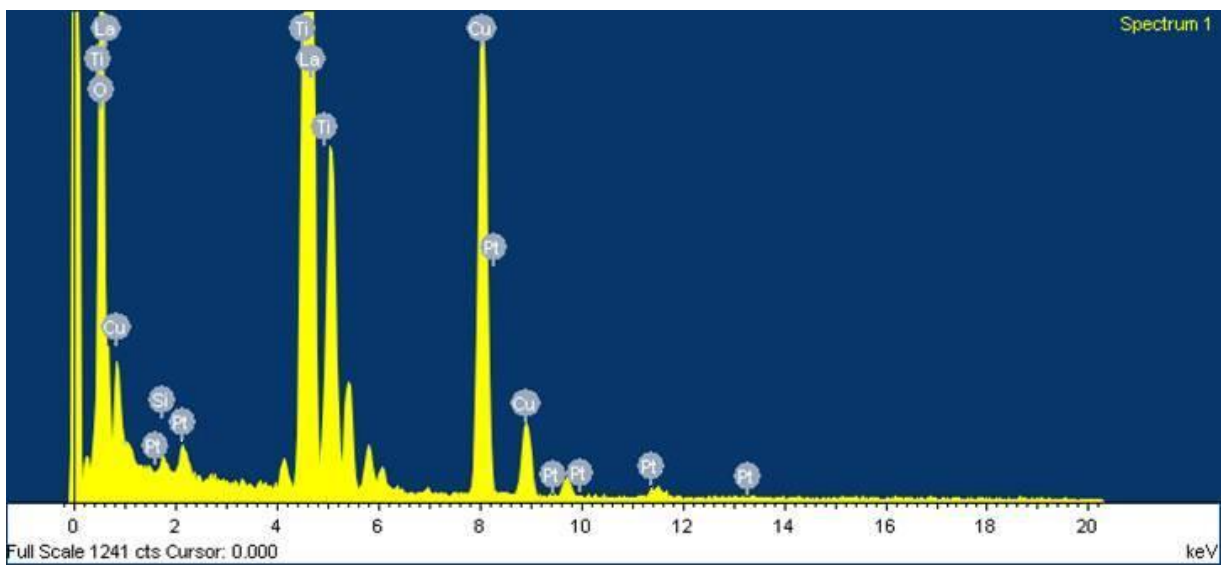

Figure 5-2 EDX spectrum obtained from the nitrogen doped $\mathrm{La}_{2} \mathrm{Ti}_{2} \mathrm{O}_{7}$ nanosheets loaded with the $\mathrm{Pt}$ nanoparticles.

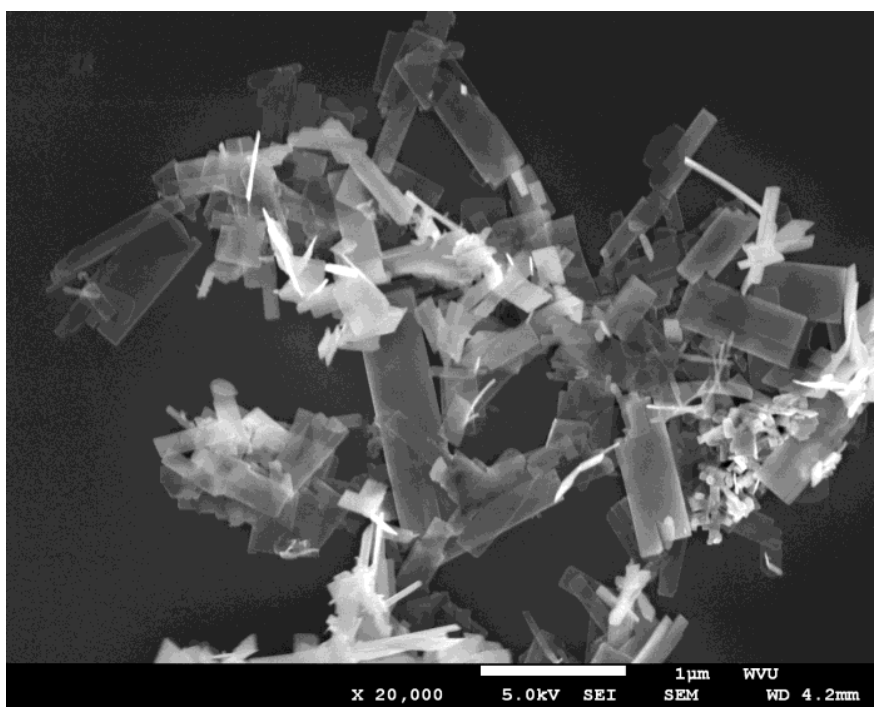

Figure 5-3 SEM image of the nitrogen-doped $\mathrm{La}_{2} \mathrm{Ti}_{2} \mathrm{O}_{7}$ nanosheets.

Figure 5-1 (a) shows the SEM image of the pristine LTO. The nanosheets were around $500 \mathrm{~nm}$ in size. The LTO nanosheets had a specific surface area of $37.20 \mathrm{~m}^{2} / \mathrm{g}$, which was measured by the BET method. After impregnation in the Pt-containing precursor, $5 \mathrm{~nm}$ sized nanoparticles were distributed on the surface of the LTO nanosheets homogeneously as shown in the TEM image (Figure 5-1 (b)). EDX analysis 
further confirmed that these dark nanoparticles were Pt nanoparticles as shown in Figure 5-2. Furthermore, the HRTEM image in Figure 5-1 (c) showed one of the Pt nanoparticles on the LTO nanosheet surface. The lattice fringe with a spacing of 0.195 $\mathrm{nm}$ in the dark area corresponded to Pt $(200)$. The lattice fringe with a spacing of 0.299 $\mathrm{nm}$ was attributed to LTO $\left(\begin{array}{lll}-2 & 1 & 2\end{array}\right)$. Therefore, it was concluded that metallic Pt nanoparticles was successfully deposited on the LTO nanosheet surface. After nitrogen doping, the nanosheets structure of LTO was preserved as shown in Figure 5-3, which retained high specific surface area and active catalytic sites. Figure 5-1 (d) shows the XRD patterns of P-LTO and Pt@N-LTO. It can be seen that nitrogen doping did not change the crystal phase of LTO nanosheets. After loading with Pt, a small peak at $46.24^{\circ}$ on the left of the LTO ( $\left.\begin{array}{lll}-1 & 0 & 4\end{array}\right)$ peak was attributed to Plane $\left(\begin{array}{lll}2 & 0 & 0\end{array}\right)$ of metallic Pt. The diffraction intensity of the Pt peak was weak due to the small amount and the small size of the Pt nanoparticles.
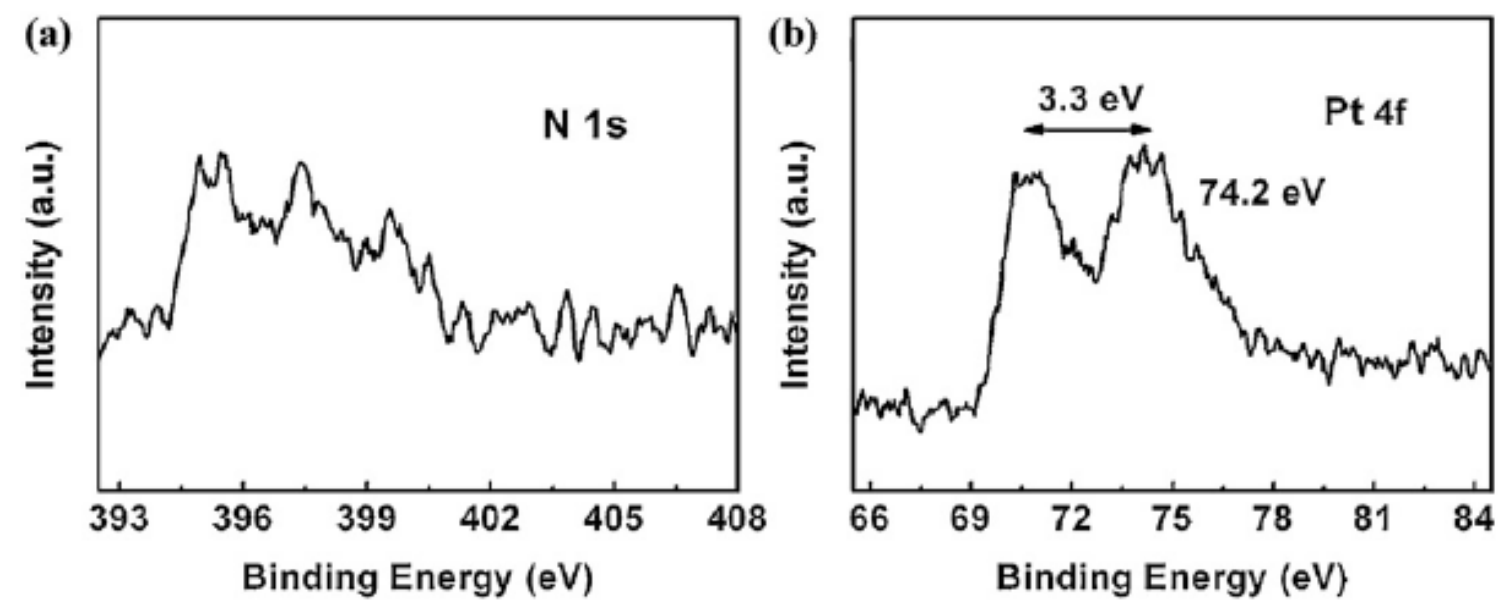

Figure 5-4 XPS spectra of $\mathrm{N} 1 \mathrm{~s}(\mathrm{a})$ and Pt $4 \mathrm{f}(\mathrm{b})$ of the nitrogen-doped $\mathrm{La}_{2} \mathrm{Ti}_{2} \mathrm{O}_{7}$ nanosheets loaded with Pt. 
XPS was used to determine the chemical status of doped nitrogen and loaded Pt in the Pt@N-LTO sample. The nitrogen content was estimated to be 2.0 at. \%. A broad and symmetric N 1s peak was observed between $395 \mathrm{eV}$ and $400 \mathrm{eV}$ (Figure 5-4 (a)). The N 1s peak consisted of several components due to different chemical statuses of the $\mathrm{N}$ atoms, which were analyzed in our previous study [7]. The XPS peak in Figure 5-4 (b) was characteristic of the Pt $4 \mathrm{f}$ core-level with a gap of $3.3 \mathrm{eV}$ between $\mathrm{Pt} 4 \mathrm{f} 5 / 2$ and $\mathrm{Pt}$ 4f7/2. The Pt 4f7/2 peak was located at $74.2 \mathrm{eV}$, which confirmed metallic Pt on the NLTO nanosheets [17].

\subsubsection{Light absorption of photocatalysts}

The light absorption property of the nanosheets was evaluated by UV-Vis spectroscopy (Figure 3). For P-LTO, the optical absorption edge was cut off at around $330 \mathrm{~nm}$, corresponding to a band gap of $3.76 \mathrm{eV}$. The optical absorption edge shifted to around $560 \mathrm{~nm}$ after nitrogen doping. The band gap of N-LTO was estimated to be 2.20 $\mathrm{eV}$ based on the optical absorption edge. Nitrogen doping led to a red shift of the band gap up to $1.56 \mathrm{eV}$. It is worth noting that the red shift of light absorption of the N-LTO nanosheets was completely different from that of the nitrogen-doped $\mathrm{TiO}$ 2. In $\mathrm{N}$-doped $\mathrm{TiO} 2$, an add-on shoulder closely followed the cutoff edge of the absorption due to the localized $\mathrm{N} 2 \mathrm{p}$ orbitals near the valence band maximum [5]. However, the N-LTO exhibited a parallel shift of the optical absorption edge rather than the add-on shoulder, which was attributed to band gap narrowing [7]. Loading Pt on the N-LTO did not cause any significant change in the absorption spectrum. The P-LTO power was white while the N-LTO became yellow, which also suggested the enhanced visible light absorption after 
nitrogen doping. There was no evident difference in the color between N-LTO and Pt@N-LTO.

\subsubsection{Photocatalytic hydrogen generation}

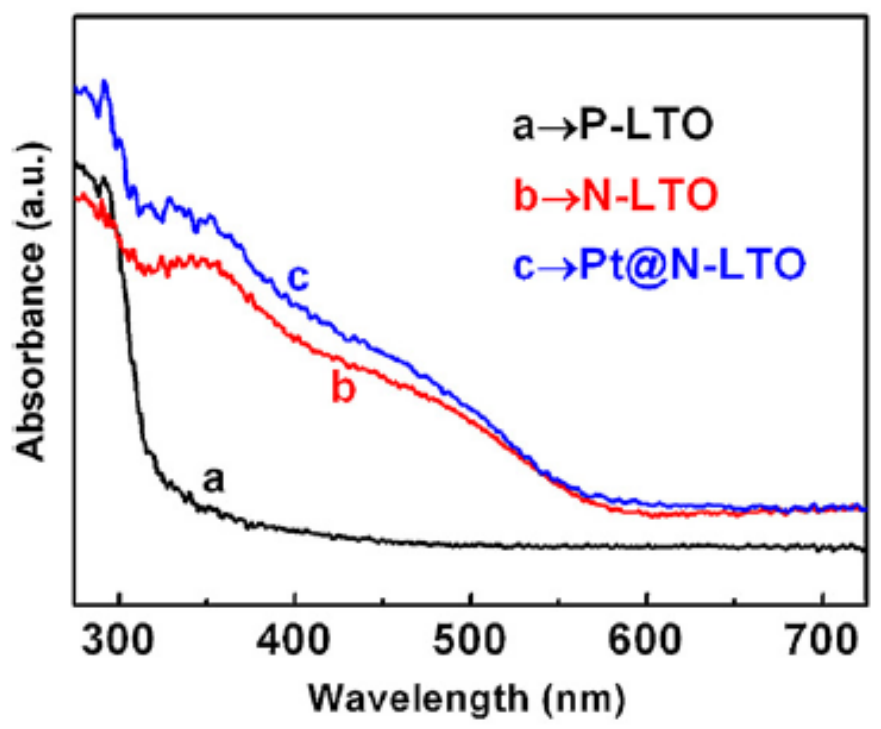

Figure 5-5 UV-vis absorption spectra of the pristine $\mathrm{La}_{2} \mathrm{Ti}_{2} \mathrm{O}_{7}$ nanosheets (a), the nitrogen-doped nanosheets (b), and the Pt-loaded nitrogen-doped nanosheets (c).

The photocatalytic water-splitting activity was monitored by measuring the amount of $\mathrm{H} 2$ produced under the visible light and the UV irradiation, respectively. Figure 5-5 (a) shows the $\mathrm{H} 2$ production under visible light irradiation after different time intervals $(5,10,15$ and 20 h). Neither P-LTO nor Pt@P-LTO displayed the photocatalytic activity toward water splitting. The photon energy of the incident visible light centered at $420 \mathrm{~nm}$ was insufficient to overcome the energy barrier of charge separation due to the large band gap of pristine LTO $(3.76 \mathrm{eV})$. Since nitrogen doping 
narrowed the band gap down to $2.2 \mathrm{eV}$, the incident visible light can excite electrons and holes in N-LTO, leading to significant photocatalytic activity toward water-splitting.
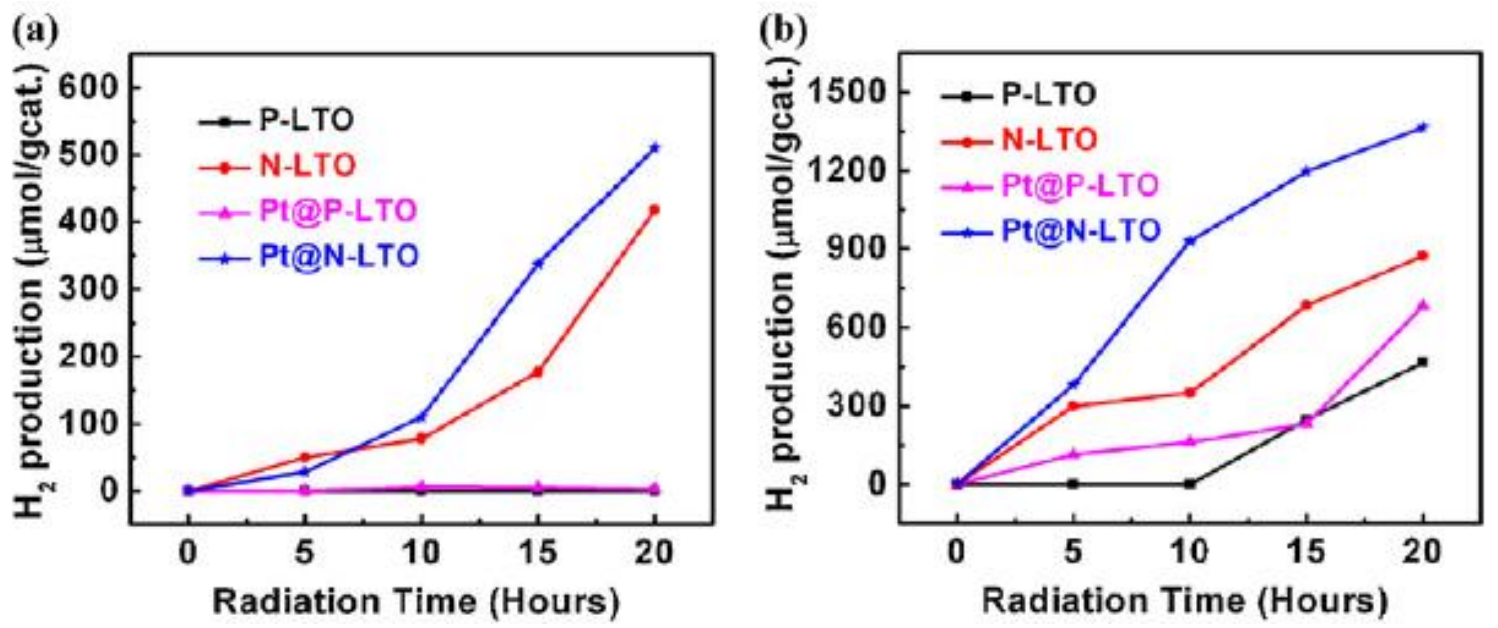

Figure 5-6 Hydrogen production by four samples under the visible light (a) and the ultraviolet (b) irradiation.

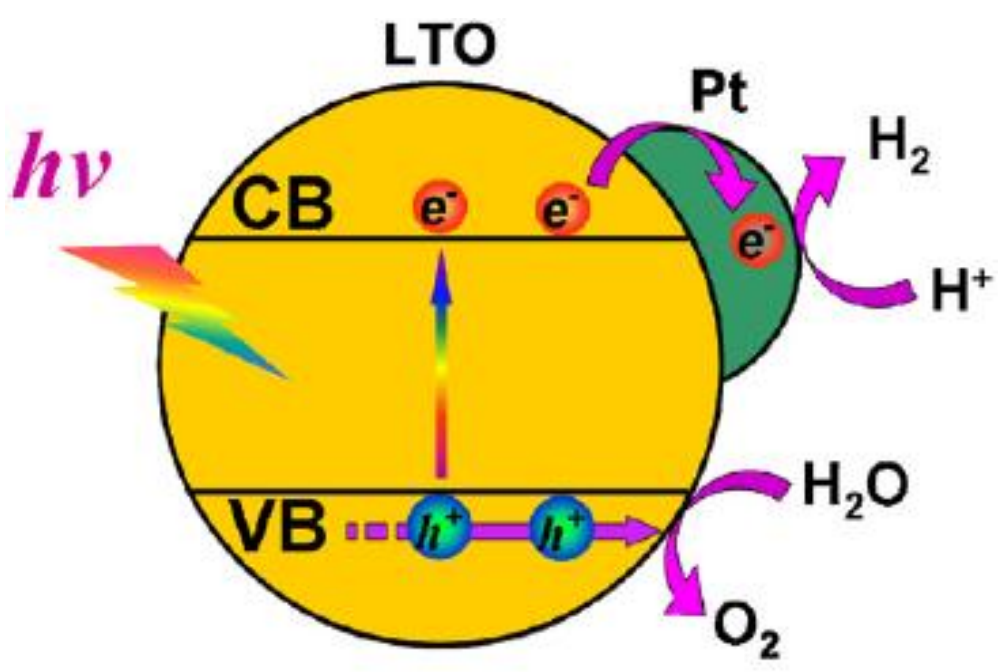

Figure 5-7 Schematic of photocatalytic hydrogen generation.

Pt-loading enhanced the photocatalytic activity under both the visible light (Figure 5-6 (a)) and the UV light (Figure 5-6 (b)) irradiation. In order to understand the 
underlying mechanism, a scheme was drawn to illustrate the process of photocatalytic water-splitting (Figure 5-7). Electrons in LTO are excited to the conduction band (CB) by the incident photons, leaving holes in the valence band (VB). Electrons migrate to the photocatalyst surface and to react with $\mathrm{H}^{+}$, producing $\mathrm{H}_{2}$. In the meanwhile, the accumulated holes transport to the photocatalyst surface to form $\mathrm{O}_{2}$. If the photogenerated electrons do not move out quickly, some of them will recombine with holes. When Pt is present on LTO surface, the Fermi levels of metal Pt and the LTO are aligned. This Fermi level alignment would facilitate the electron transfer from the LTO to the reaction site, and thus suppress the recombination between the photogenerated electrons and holes. Therefore, Pt-loading can enhance the photocatalytic activity toward water splitting for both P-LTO and N-LTO. Under the UV light irradiation, all four samples exhibited the photocatalytic activity toward water splitting as shown in Figure 56 (b). The N-doped samples showed much better photocatalytic activity than that of the undoped samples because the N-doped samples had much narrower band gap. Therefore, the efficiency of the charge separation was much higher.

\subsection{Conclusions}

The pristine LTO nanosheets were synthesized by a hydrothermal method. $5 \mathrm{~nm}$ sized Pt nanoparticles were homogeneously dispersed on the surface of LTO. Nitrogen was doped into the lattice of LTO nanosheets while preserving the nanosheet morphology. Nitrogen doping extended the light absorption up to $560 \mathrm{~nm}$, enabling the photocatalytic activity toward water splitting under visible light irradiation. Loading the Pt nanoparticles 
on the LTO surface further improved the photocatalytic activity due to the enhanced separation of charge carriers.

\section{Reference}

[1] Fujishima, A.; Honda, K. Nature, 1972, 238, 37-38.

[2] Kudo, A.; Miseki,Y. Chem. Soc. Rev. 2009, 38, 253.

[3] Abe, R., Higashi, M., Sayama, K., Abe, Y., Sugihara, H., J. Phys. Chem. B., 2006, 110, 2219.

[4] Tafen, D, Wang, J., Wu, N. Q., Lewis, J. P., Appl. Phys. Lett., 2009, 94, 093101.

[5] Wang, J., Tafen, D. N., Lewis, J. P., Hong, Z. L., Manivannan, A., Zhi, M. J., Li, M., Wu, N. Q., J. Am. Chem. Soc., 2009, 131, 12290.

[6] N.Q. Wu, J. Wang, D.N. Tafen, H. Wang, J.G. Zheng, J.P. Lewis, X.G. Liu, S.S.

Leonard, A. Manivannan, J. Am. Chem. Soc., 2009, 131, 12290.

[7] F.K. Meng, H.Z. Lian, J. Arndt, M. Li, M.J. Zhi, F. Yang, N.Q. Wu, Nano Research, 2012, 5 213-221.

[8] Jeong. H, Kim. T, Kim. D, Kim. K, Int. J. Hydrogen Energ, 2006, 31, 1142.

[9] Takata. T, Furumi. Y, Shinohara. K, Tanaka. A, Hara. M, Kondo. J. N, Domen. K, Chem. Mater., 1997, 9, 1063.

[10] Kim. H. G, Hwang. D. W, Kim. J, Kim. Y. J, Lee. J. S, Chem. Commun., 1999, 12, 1077.

[11] Hwang, D. W., Kim, H. G., Lee, J. S., Kim, J., Li, W., Oh, S. H., J. Phys. Chem. B., 2005, 109, 2093. 
[12] Kim, J. D., Hwang, D. W., Kim, H. G., Bae, S. W., Lee, J. S., Li, W., Oh, S. H., Top. Catal., 2005, 35, 295.

[13] Kasahara, A., Nukumizu, K., Takata, T., Kondo, J. N., Hara, M., Kobayashi, H., Domen, K., J. Phys. Chem. B., 2003, 107, 791.

[14] Li, K.; Wang, Y.; Wang, H.; Zhu M.; and Yan, H. Nanotech. 2006 17, 4863.

[15] Kasahara, A., Nukumizu, K., Hitoki, G., Takata, T., Kondo, J. N., Hara, M.,

Kobayashi, H., Domen, K., J. Phys. Chem. A., 2002, 106, 6750.

[16] Sathish, M., Viswanathan, B., Viswanath, R. P., Gopinath, S., Chem. Mater., 2005, $17,6349$.

[17] J.F. Moulder, W.F. Stickle, P.E. Sobol, K.D. Bomben, Handbook of X-ray Photoelectron Spectroscopy, Eden Prairie, 1992. 


\section{Chapter 6 Photocatalytic Water Oxidation by}

\section{Hematite/Reduced Graphene Oxide Composite}

\subsection{Background and introduction}

Energy challenges have inspired the development of inexpensive and active materials for solar fuel production by photocatalytic water splitting [1-3]. In Chapters 3-5 the performance of large band gap metal oxide photocatalysts was enhanced by doping without the introduction of mid-gap states. However, another class of materials exists for photocatalysis. These small band gap materials do not suffer from poor spectral coverage, but instead suffer from high charge recombination and poor charge migration. Therefore a different enhancement strategy must be developed opposite of that for large band gap materials where the opposite situation exists.

Hematite $\left(\alpha-\mathrm{Fe}_{2} \mathrm{O}_{3}\right)$ stands out among many promising candidate materials because of its low cost and favorable band gap (2.1-2.2 eV) to absorb photons in the visible light range $[4,5]$. However, its photocatalytic efficiency is hindered by the short lifetime of the photogenerated charge carriers $(<10 \mathrm{ps})$, short hole diffusion length $(2-4$ $\mathrm{nm})$, and poor mobility of charge carriers $\left(<0.2 \mathrm{~cm}^{2} \cdot \mathrm{V}^{-1} \cdot \mathrm{s}^{-1}\right)[5,6]$. To address these limitations, $\alpha-\mathrm{Fe}_{2} \mathrm{O}_{3}$ should be created as nanostructures to ensure that the charge carriers produced deep inside the $\alpha-\mathrm{Fe}_{2} \mathrm{O}_{3}$ are able to diffuse to the hematite/electrolyte interface [6,7]. Many methods have been developed to engineer various morphologies of $\alpha-\mathrm{Fe}_{2} \mathrm{O}_{3}$ $[8,9]$. Nevertheless, $\alpha-\mathrm{Fe}_{2} \mathrm{O}_{3}$ still suffers from a high charge recombination rate because of boundaries between the particles. $\alpha-\mathrm{Fe}_{2} \mathrm{O}_{3}$ nanowires have shown the enhanced 
photoelectrochemical cell (PEC) performance [10,11] because one- or two-dimensional nanostructures generally have higher mobility and lower charge recombination rates than zero dimensional nanoparticles [12,13]. However, the short hole diffusion length still limits the efficiency of charge carrier transport along the axial direction of the nanowires. Alternatively, $\alpha-\mathrm{Fe}_{2} \mathrm{O}_{3}$ has been combined with charge extraction layers to improve the charge separation by providing a more mobile hole pathway; the typical example being cobalt/phosphate catalyst or $\mathrm{IrO}_{2}$ deposited onto $\alpha-\mathrm{Fe}_{2} \mathrm{O}_{3}$ photoanodes $[4,14]$. Also, a p-n junction could effectively extract holes from $\alpha-\mathrm{Fe}_{2} \mathrm{O}_{3}$ and improves the PEC performance $[2]$.

In contrast, coupling $\alpha-\mathrm{Fe}_{2} \mathrm{O}_{3}$ with an electron acceptor is rare [15]. Graphene or reduced graphene oxide ( $\mathrm{rGO}$ ) are good candidates because of the high electron mobility $\left(>15000 \mathrm{~cm}^{2} \cdot \mathrm{V}^{-1} \cdot \mathrm{s}^{-1}\right)$ and the flexible sheet nature that is beneficial to support photocatalysts [16]. The previous studies have shown that the incorporation of graphene (or rGO) with metal oxide can enhance the photocatalytic activity $[15,16]$. It is claimed that photocatalysis enhancement by graphene is due to the fact that graphene provides a pathway for transport of charge carriers. However, no direct experimental evidence has yet been provided to prove this point. The graphene-enhanced photocatalysis mechanism remains poorly understood.

Herein, $\alpha-\mathrm{Fe}_{2} \mathrm{O}_{3} / \mathrm{rGO}$ composites in which hematite nanoparticles are supported on the rGO nanosheets are synthesized by a hydrothermal process. The $\alpha-\mathrm{Fe}_{2} \mathrm{O}_{3} / \mathrm{rGO}$ composite shows the enhanced photocatalytic activity toward water oxidation compared with the pristine $\alpha-\mathrm{Fe}_{2} \mathrm{O}_{3}$ nanoparticles. Transient absorption and time-domain terahertz spectroscopy provide the direct evidence for the first time that electrons can transfer as 
the mobile carriers from $\alpha-\mathrm{Fe}_{2} \mathrm{O}_{3}$ into the $\mathrm{rGO}$, which can diffuse and become trapped, thus reducing the charge recombination rate.

\subsection{Experimental section}

\subsubsection{Material synthesis}

Graphene oxide (GO) was synthesized by the established Hummer method [17]. The $\alpha-\mathrm{Fe}_{2} \mathrm{O}_{3} /$ reduced graphene oxide (rGO) was prepared by the hydrothermal method in a previous report [18]. Briefly, a proper amount of $\mathrm{FeCl}_{3} \cdot 6 \mathrm{H}_{2} \mathrm{O}(97 \%$, Alfa Aesar) was mixed with $44 \mathrm{mg}$ of GO, and then the mixture was dissolved into $200 \mathrm{~mL}$ of deionized water (DI water, $18.2 \mathrm{M} \Omega \cdot \mathrm{cm}$ at $25^{\circ} \mathrm{C}$ ) and sonicated for $30 \mathrm{~min}$. Then, $200 \mathrm{~mL}$ of ethanol (94-96\%, Alfa Aesar) was mixed with the prepared solution and placed in a boiling aqueous bath for $2 \mathrm{~h}$ for thermal hydrolysis. Consequently, the $\alpha-\mathrm{Fe}_{2} \mathrm{O}_{3} / \mathrm{rGO}$ sample was collected by centrifuging at a rate of $4000 \mathrm{rpm}$. Finally, the sample was heated in air at $350{ }^{\circ} \mathrm{C}$ for $2 \mathrm{~h}$ and then in pure nitrogen at $800{ }^{\circ} \mathrm{C}$ for $15 \mathrm{~min}$. The $\alpha-$ $\mathrm{Fe}_{2} \mathrm{O}_{3} / \mathrm{rGO}$ ratio was adjusted by varying the amount of hematite precursor added during the hydrothermal process. Three ratios $\left(1,2\right.$, and $3 \mathrm{~g}$ of $\left.\mathrm{FeCl}_{3} \cdot 6 \mathrm{H}_{2} \mathrm{O}\right)$ were employed in this study. According to the content of the rGO, the resulting samples were denoted as $\alpha$ $\mathrm{Fe}_{2} \mathrm{O}_{3} / \mathrm{rGO}(\mathrm{h})$ with a high $\mathrm{rGO}$ content, $\alpha-\mathrm{Fe}_{2} \mathrm{O}_{3} / \mathrm{rGO}$ with a medium $\mathrm{rGO}$ content, and $\alpha-$ $\mathrm{Fe}_{2} \mathrm{O}_{3} / \mathrm{rGO}(\mathrm{l})$ with a low rGO content, respectively. For preparation of monolithic $\alpha-$ $\mathrm{Fe}_{2} \mathrm{O}_{3}$, no $\mathrm{GO}$ was added into the solution, and the heating process was directly conducted in air at $800{ }^{\circ} \mathrm{C}$ for $15 \mathrm{~min}$. A physical mixture of $\alpha-\mathrm{Fe}_{2} \mathrm{O}_{3}$ and $\mathrm{rGO}(\alpha-$ 
$\mathrm{Fe}_{2} \mathrm{O}_{3}+\mathrm{rGO}$ ) was also prepared as a control sample to measure the photocatalytic water oxidation performance.

\subsubsection{Characterizations and light absorption measurement}

The morphologies of $\alpha-\mathrm{Fe}_{2} \mathrm{O}_{3}$ and $\alpha-\mathrm{Fe}_{2} \mathrm{O}_{3} / \mathrm{rGO}$ were observed by a fieldemission scanning electron microscope (SEM, JEOL 7600F) and a transmission electron microscope (TEM, JEOL JEM 2100F) respectively. For SEM sample preparation, 2 mg of powder sample was mixed in $1 \mathrm{~mL}$ of deionized water, which was then placed in an ultrasonic bath for $30 \mathrm{~s}$ to obtain a homogeneous suspension. Consequently, the suspension was deposited onto a precleaned silicon wafer substrate $(1 \mathrm{~cm} \times 1 \mathrm{~cm}$, SPI Inc.), and then dried in air at $50{ }^{\circ} \mathrm{C}$. For TEM sample preparation, the powder was suspended in ethanol and dropped onto a TEM grid (Ted Pella Inc.), and then dried at room temperature overnight. The crystal structures of $\alpha-\mathrm{Fe}_{2} \mathrm{O}_{3}$ and $\alpha-\mathrm{Fe}_{2} \mathrm{O}_{3} / \mathrm{rGO}$ were characterized by a high-resolution TEM (HRTEM) and X-ray diffraction (XRD, $\mathrm{X}^{\prime}$ Pert Pro PW3040-Pro, Panalytical Inc.) with $\mathrm{Cu} \mathrm{K \alpha}$ radiation. X-ray photoelectron spectroscopy (XPS, PHI 5000 Versa Probe System, Physical Electronics, MN) was used for determining the chemical status of $\mathrm{Fe}$ and $\mathrm{C}$. The $\mathrm{C} 1 \mathrm{~s}$ peak at $284.6 \mathrm{eV}$ worked as a reference to calibrate the binding energies in XPS spectra. Shimadzu 2550 UV-visible spectrometer equipped with an integrating sphere (UV 2401/2, Shimadzu) was used to obtain the UV-visible absorption spectra under the diffuse reflection mode. The typical sample preparation for the UV-visible spectrometer was pressing the sample powder (0.3-0.5 g) onto a pre-pressed $\mathrm{BaSO}_{4}$ paste to form a solid pellet. The Fourier transform infrared spectra (FTIR) were obtained from the $\mathrm{KBr}$ pellet containing the $\alpha-\mathrm{Fe}_{2} \mathrm{O}_{3}$ and the 
$\alpha-\mathrm{Fe}_{2} \mathrm{O}_{3} / \mathrm{rGO}$ samples under transmission mode with a Nicolet 6700 spectrometer (Thermo Scientific, Waltham, MA). The surface areas of all the samples were measured by the Brunauer-Emmett-Teller (BET) method with the instrument of Micromeritics ASAP 2020. The content of rGO was measured by Thermogravimetric Analysis (TGA, TA Instrument Q50).

\subsubsection{Photocatalytic water oxidation testing}

The simulated sunlight (light intensity: $80 \mathrm{~mW} / \mathrm{cm}^{2}$ ) was generated by a commercial xenon lamp solar simulator (300 W, Newport) equipped with an AM 1.5G filter. The $\alpha-\mathrm{Fe}_{2} \mathrm{O}_{3}$ and $\alpha-\mathrm{Fe}_{2} \mathrm{O}_{3} / \mathrm{rGO}$ samples were used for oxygen generation by photocatalytic water splitting as follows. First, DI water was boiled on a hot plate for 10 min to ensure all oxygen dissolved into the water was bubbled out. Second, the oxygenfree DI water and a magnetic stirring bar were added in a $100 \mathrm{~mL}$ quartz flask, which was then sealed with a septum stopper. Third, pure nitrogen was injected into the flask to squeeze out $85 \mathrm{~mL}$ of DI water. Fourth, $6 \mathrm{mg}$ of the $\alpha-\mathrm{Fe}_{2} \mathrm{O}_{3}$ or $\alpha-\mathrm{Fe}_{2} \mathrm{O}_{3} / \mathrm{rGO}$ samples and $68 \mathrm{mg}$ of $\mathrm{AgNO}_{3}(>99 \%$, Sigma-Aldrich) were mixed with $5 \mathrm{~mL}$ of the oxygen-free DI water and ultrasonicated for $30 \mathrm{~s}$ and then injected into the flask, so the total liquid in the flask was $20 \mathrm{~mL}$. Fifth, the flask was then placed in an ultrasonic bath for another $10 \mathrm{~min}$ to form a homogeneous suspension. Sixth, the flask was exposed to the light for $4,8,12$, 16 , and $20 \mathrm{~h}$, respectively. Finally, $5 \mathrm{~mL}$ of gas sample was extracted from the flask to measure the gas composition at each reaction time with a gas chromatograph (GC) equipped with a thermal conductivity detector (TCD). 
The stability of the photocatalytic water oxidation was evaluated by measuring the rate of photocatalytic $\mathrm{O}_{2}$ evolution in four cycles of the re-collected samples. Between each cycle, the photocatalyst was collected by centrifuging the suspension and then redissolved into $0.02 \mathrm{M} \mathrm{AgNO}_{3}$ solution and sealed into a quartz flask. The detail procedure was identical as that described above.

\subsubsection{Photoelectrochemical measurement}

First, $0.1 \mathrm{~g}$ of $\alpha-\mathrm{Fe}_{2} \mathrm{O}_{3}$ or $\alpha-\mathrm{Fe}_{2} \mathrm{O}_{3} / \mathrm{rGO}$ samples were mixed with $0.5 \mathrm{~mL}$ of terpineol ( $~ 95 \%$, Sigma-Aldrich) and stirred on a magnetic stirrer for $24 \mathrm{~h}$. Second, the terpineol suspension was coated onto fluorine-doped tin oxide (FTO) glass substrates (Hartford, TEC 15) with the doctor blading technique to prepare a series of photoelectrodes under the similar conditions to ensure the same thickness of each photoelectrode. Third, the $\alpha-\mathrm{Fe}_{2} \mathrm{O}_{3}$ or $\alpha-\mathrm{Fe}_{2} \mathrm{O}_{3} / \mathrm{rGO}$ photoelectrodes were dried on a hot plate and then transferred to a quartz tubular furnace to sinter in nitrogen at $550{ }^{\circ} \mathrm{C}$ for 2 h. Fourth, a silver wire was linked on the FTO with silver paste and then the epoxy was solidified onto the sample-uncovered areas on FTO substrate to avoid short current in the following electrochemical measurement. Photoelectrochemical measurement was conducted with a three-electrode cell using the prepared photoanodes as the working electrode, $\mathrm{Pt}$ wire as the counter electrode, and $\mathrm{Ag} \mid \mathrm{AgCl}$ as the reference electrode. As the electrolyte, $1 \mathrm{~mol} / \mathrm{L} \mathrm{NaOH}(>98.0 \%$, Sigma-Aldrich) aqueous solution $(\mathrm{pH}=13.6)$ was bubbled for $30 \mathrm{~min}$ with $\mathrm{N}_{2}$ prior to measurement. The identical light source used in the photocatalysis experiments was applied to illuminate the samples. The J-V curve was acquired with a Gamry electrochemical station (Reference 3000). The obtained potentials 
vs $\mathrm{Ag} \mid \mathrm{AgCl}$ can be converted to the reversible hydrogen electrode (RHE) according to the following Nernst equation 6-1:

$$
E_{\text {RHE }}=E+0.05916 p H+E_{0}
$$

where Erhe is the potential vs RHE, $\mathrm{E}_{0}=0.1976 \mathrm{~V}$ at $25{ }^{\circ} \mathrm{C}$, and $\mathrm{E}$ is the measured potential vs $\mathrm{Ag} \mid \mathrm{AgCl}$.

\subsection{Results and discussion}

\subsubsection{Morphology and crystal structure}

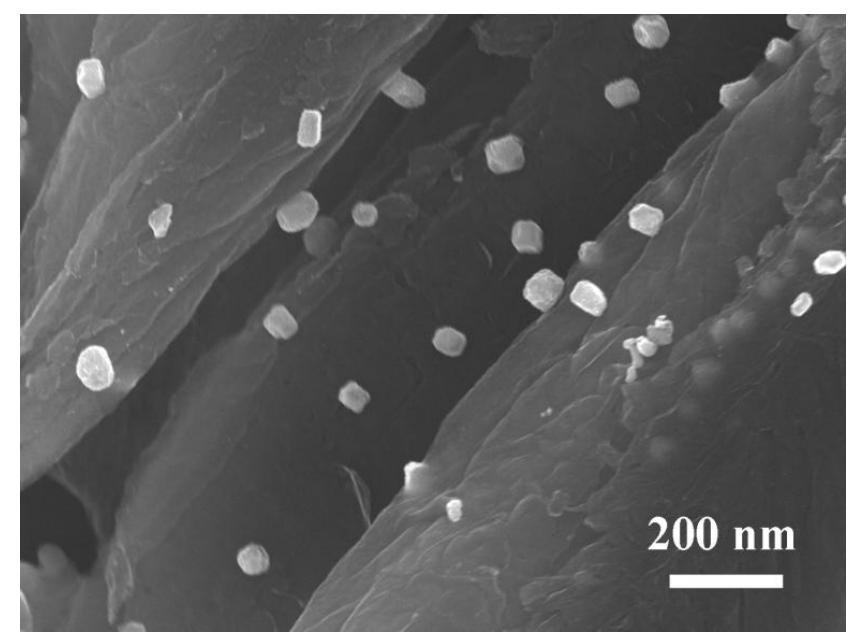

Figure 6-1. SEM image of the $\alpha-\mathrm{Fe}_{2} \mathrm{O}_{3} / \mathrm{rGO}(\mathrm{h})$ composite containing $6.1 \mathrm{wt} \%$ of reduced graphene oxide (rGO) after heat treatment at $800^{\circ} \mathrm{C}$. 


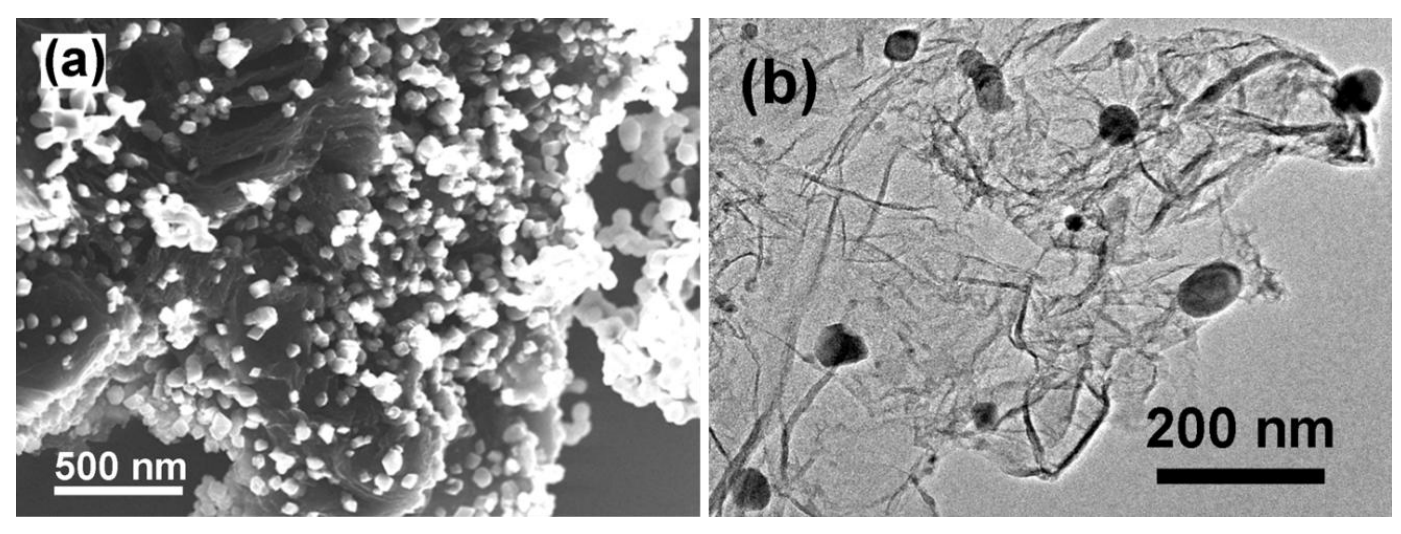

Figure 6-2. (a) SEM and (b) TEM images of the $\alpha-\mathrm{Fe}_{2} \mathrm{O}_{3} / \mathrm{rGO}$

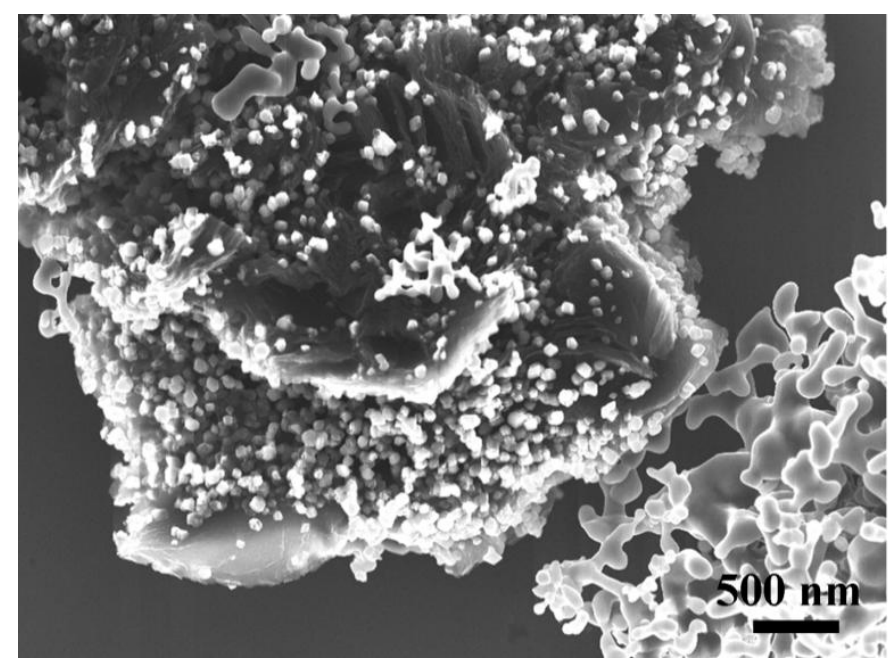

Figure 6-3. SEM image of the $\alpha-\mathrm{Fe}_{2} \mathrm{O}_{3} / \mathrm{rGO}$ (l) composite containing $2.9 \mathrm{wt} \%$ of reduced graphene oxide (rGO) after heat treatment at $800{ }^{\circ} \mathrm{C}$. 

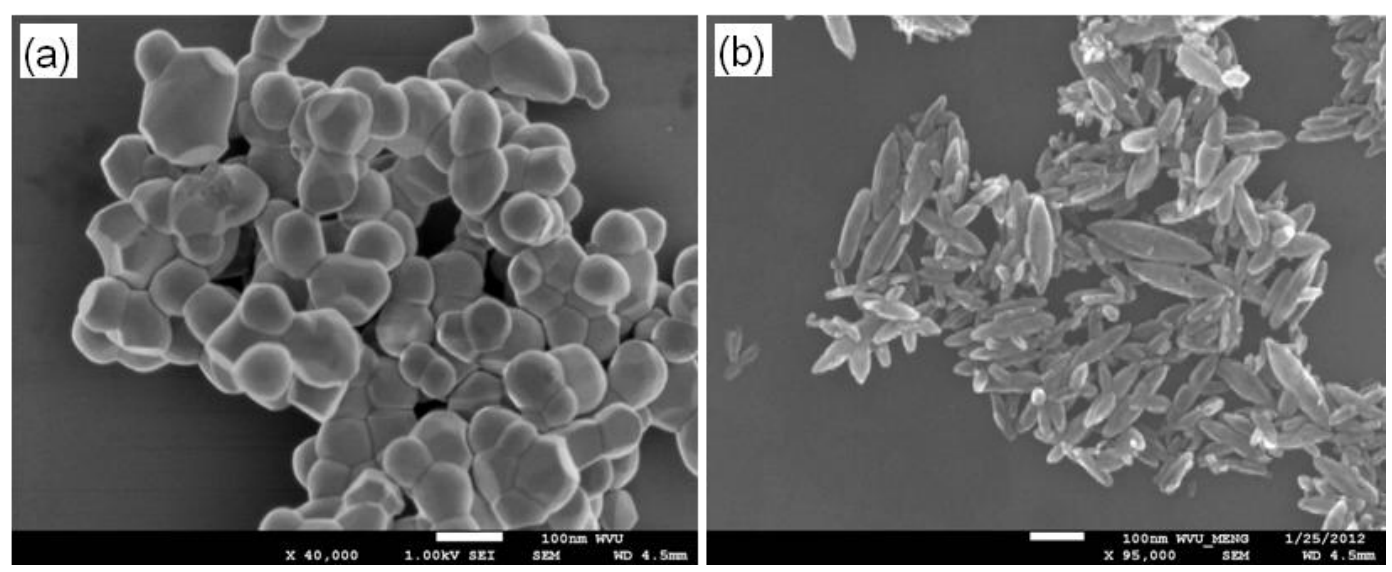

Figure 6-4. SEM image of pristine $\alpha-\mathrm{Fe}_{2} \mathrm{O}_{3}$; (a) after, and (b) before heat treatment at $800{ }^{\circ} \mathrm{C}$.

Four types of samples were prepared by the hydrothermal processing and the follow-up heat treatment: (a) the $\alpha-\mathrm{Fe}_{2} \mathrm{O}_{3} / \mathrm{rGO}(\mathrm{h})$ composites containing 6.1 wt $\%$ of reduced graphene oxide (rGO) as shown in Figure 6-1, (b) the $\alpha-\mathrm{Fe}_{2} \mathrm{O}_{3} / \mathrm{rGO}$ composites containing 3.8 wt $\%$ of rGO as shown in Figure $6-2$, (c) the $\alpha-\mathrm{Fe}_{2} \mathrm{O}_{3} / \mathrm{rGO}(\mathrm{l})$ composites containing 2.9 wt $\%$ of rGO as shown in Figure 6-3, and (d) pristine $\alpha-\mathrm{Fe}_{2} \mathrm{O}_{3}$ as shown in Figure 6-4. The rGO contents in the $\alpha-\mathrm{Fe}_{2} \mathrm{O}_{3} / \mathrm{rGO}$ composites were calculated from the TGA data in Figure 6-5. 


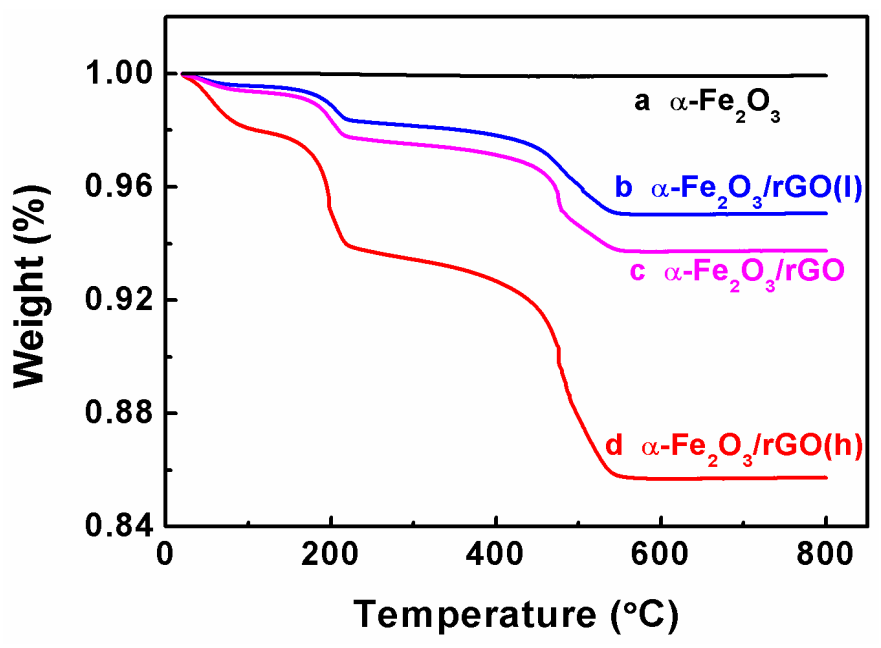

Figure 6-5. TGA plots; (a) $\alpha-\mathrm{Fe}_{2} \mathrm{O}_{3}$, (b) $\alpha-\mathrm{Fe}_{2} \mathrm{O}_{3} / \mathrm{rGO}$ (l) containing 2.9 wt $\%$ of $\mathrm{rGO}$, (c) $\alpha-\mathrm{Fe}_{2} \mathrm{O}_{3} / \mathrm{rGO}$ containing $3.8 \mathrm{wt} \%$ of $\mathrm{rGO}$, and (d) $\alpha-\mathrm{Fe}_{2} \mathrm{O}_{3} / \mathrm{rGO}(\mathrm{h})$ containing $6.1 \mathrm{wt} \%$ of $\mathrm{rGO}$.

It can be seen that the $\alpha-\mathrm{Fe}_{2} \mathrm{O}_{3}$ sample showed a little loss in mass from room temperature to $200{ }^{\circ} \mathrm{C}$, which was apparently due to the loss of adsorbed water. The $\alpha$ $\mathrm{Fe}_{2} \mathrm{O}_{3} / \mathrm{rGO}$ composites containing different $\mathrm{rGO}$ contents demonstrated weight losses in the temperature ranges of $20-100{ }^{\circ} \mathrm{C}, 150-220^{\circ} \mathrm{C}$, and $420-550{ }^{\circ} \mathrm{C}$, respectively, which corresponded to the losses of the adsorbed water, the residual groups on the rGO, and the burnout of rGO, respectively. 


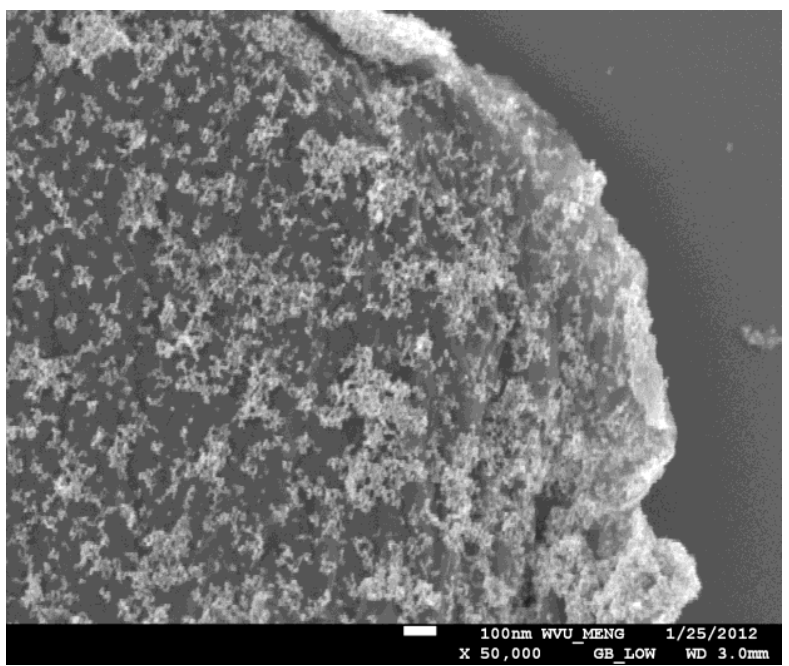

Figure 6-6. SEM image of the $\alpha-\mathrm{Fe}_{2} \mathrm{O}_{3} / \mathrm{rGO}$ before heat treatment.

SEM and TEM images in Figures 6-2 (a) and (b) show the morphology of the $\alpha$ $\mathrm{Fe}_{2} \mathrm{O}_{3} / \mathrm{rGO}$ composite. The particle sizes of $\alpha-\mathrm{Fe}_{2} \mathrm{O}_{3}$ and $\alpha-\mathrm{Fe}_{2} \mathrm{O}_{3} / \mathrm{rGO}$ increased after heating in $\mathrm{N}_{2}$ as shown in Figure 6-4, Figure 6-2 (a) and Figure 6-6.

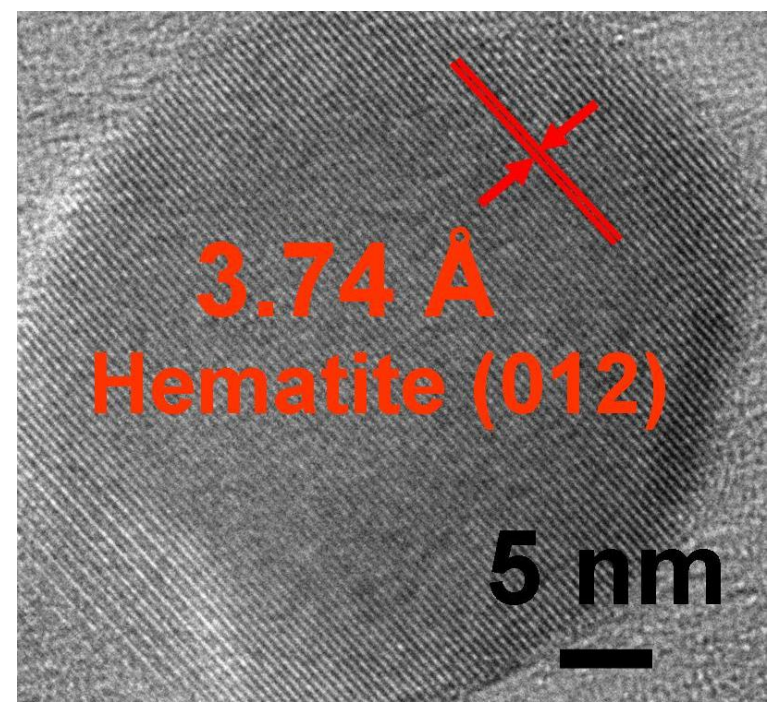

Figure 6-7 HRTEM image of one of $\alpha-\mathrm{Fe}_{2} \mathrm{O}_{3}$ nanoparticles on the rGO sheet after heat treatment at $800{ }^{\circ} \mathrm{C}$; The lattice spacing is $3.74 \AA$, which corresponded to the (012) plane of hematite (JCPDS: 33-0664) 
The TEM image of an $\alpha-\mathrm{Fe}_{2} \mathrm{O}_{3}$ particle on the $\mathrm{rGO}$ sheet in Figure 6-7 shows single crystalline structure with a lattice spacing of $3.74 \AA$, corresponding to the $(012)$ plane (JCPDS: 33-0664).

\subsubsection{Crystal structure}

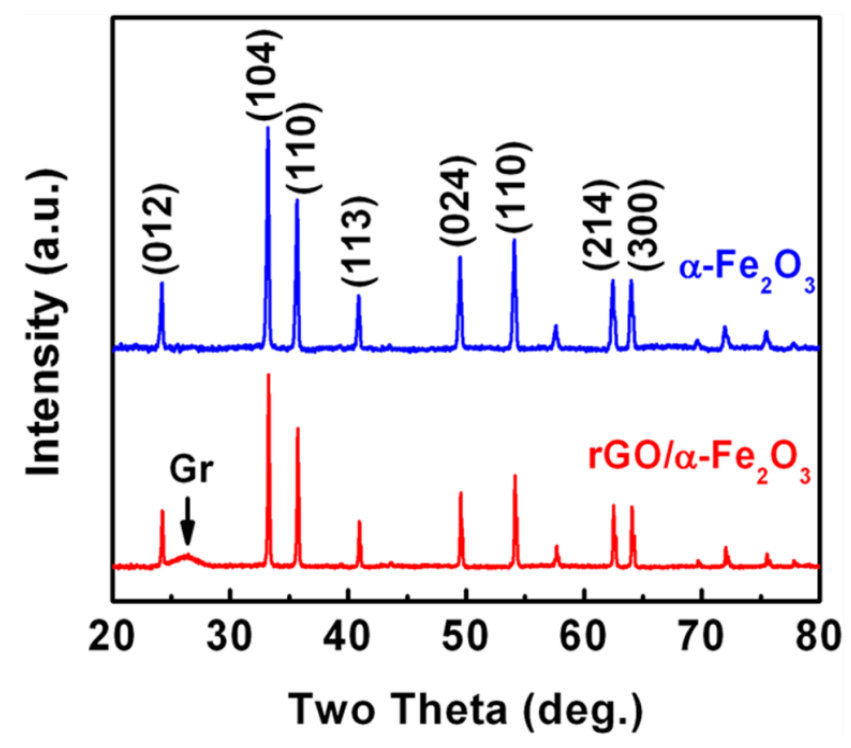

Figure 6-8. XRD patterns of the $\alpha-\mathrm{Fe}_{2} \mathrm{O}_{3}$ and $\alpha-\mathrm{Fe}_{2} \mathrm{O}_{3} / \mathrm{rGO}$

From the XRD patterns in Figure 6-8, iron oxide in both samples was indexed as hematite. The crystalline sizes were calculated based on the strong diffraction peak (104) by using the Scherrer equation. The crystalline size of monolithic $\alpha-\mathrm{Fe}_{2} \mathrm{O}_{3}(63.8 \mathrm{~nm})$ was larger than that of $\alpha-\mathrm{Fe}_{2} \mathrm{O}_{3}(41.4 \mathrm{~nm})$ grown on the rGO sheets. The broad peak located at about $27^{\circ}$ in $\alpha-\mathrm{Fe}_{2} \mathrm{O}_{3} / \mathrm{rGO}$ was assigned to be $\mathrm{rGO}[19]$. 


\subsubsection{Light absorption}

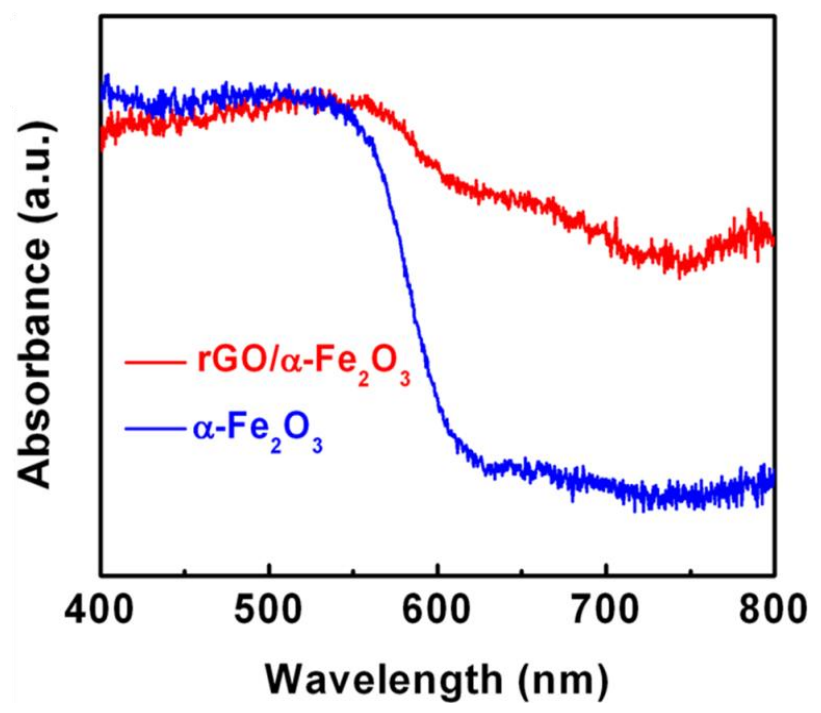

Figure 6-9. UV-visible absorption spectra of $\alpha-\mathrm{Fe} 2 \mathrm{O} 3$ and $\alpha-\mathrm{Fe} 2 \mathrm{O} 3 / \mathrm{rGO}$

The UV-visible absorption spectra in Figure 6-9 shows an absorption edge at 600 $\mathrm{nm}$, corresponding to the band gap of $\alpha-\mathrm{Fe}_{2} \mathrm{O}_{3}$ at $2.07 \mathrm{eV}$. Absorption at wavelengths longer than $600 \mathrm{~nm}$ was much stronger in the composite structure because of the presence of rGO. 


\subsubsection{FTIR and XPS results of the $\alpha-\mathrm{Fe}_{2} \mathrm{O}_{3} / \mathrm{rGO}$ composite}
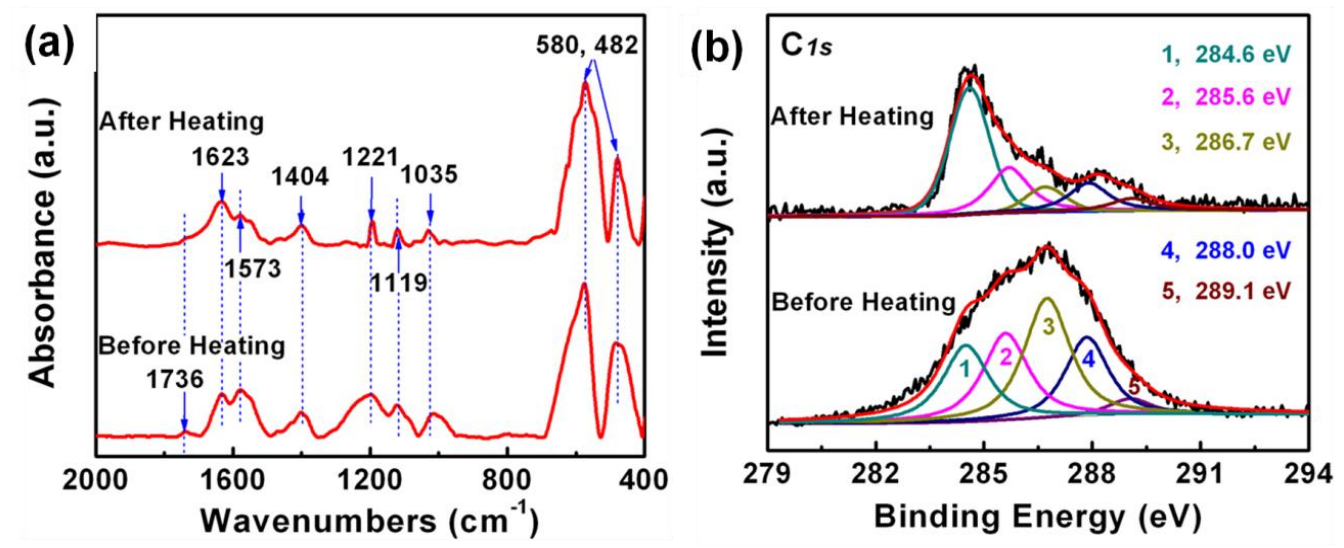

Figure 6-10. (a) FTIR spectra and (b) XPS spectra of the $\alpha-\mathrm{Fe}_{2} \mathrm{O}_{3} / \mathrm{rGO}$ before and after heating in $\mathrm{N}_{2}$ at $800{ }^{\circ} \mathrm{C}$.

Figure 6-10 (a) shows the FTIR spectra of the $\alpha-\mathrm{Fe}_{2} \mathrm{O}_{3} / \mathrm{rGO}$ composite. Signatures from the typical functional groups in the GO [20], such as $\mathrm{C}=\mathrm{O}\left(1736 \mathrm{~cm}^{-1}\right)[20-22]$ benzene ring skeletal vibration $\left(1623 \mathrm{~cm}^{-1}\right)$ [20], aromatic $\mathrm{C}=\mathrm{C}\left(1573 \mathrm{~cm}^{-1}\right)$ [20], $\mathrm{COO}-$ $\left(1404 \mathrm{~cm}^{-1}\right)[20,21], \mathrm{C}-\mathrm{OH}\left(1221 \mathrm{~cm}^{-1}\right)$ [20], $\mathrm{C}-\mathrm{O}\left(1119 \mathrm{~cm}^{-1}\right)$ [20], and $\mathrm{C}-\mathrm{O}-\mathrm{C}(1035$ $\mathrm{cm}^{-1}$ ) [20], were observed before heating. After heating, the intensities of $\mathrm{C}=\mathrm{O}, \mathrm{COO}$, $\mathrm{C}-\mathrm{OH}, \mathrm{C}-\mathrm{O}$, and $\mathrm{C}-\mathrm{O}-\mathrm{C}$ band were reduced, but the benzene ring skeletal vibration signature remained, indicating that the GO was reduced. The XPS spectra of the C 1s core level in Figure 6-10 (b) were deconvoluted into the sp2 carbon $(284.6 \mathrm{eV}), \mathrm{C}-\mathrm{O}-\mathrm{C}$ (285.6 eV), $\mathrm{C}-\mathrm{OH}$ or $\alpha-\mathrm{C}$ in $-\mathrm{C}-\mathrm{COOH}(286.7 \mathrm{eV}), \mathrm{C}=\mathrm{O}(288.0 \mathrm{eV})$ and $\mathrm{COOH}(289.1$ $\mathrm{eV})$, respectively $[20,23,24]$. The significant drops of the peaks at 285.6, 286.7, 288.0, and $289.1 \mathrm{eV}$ indicated the reduction of GO. 

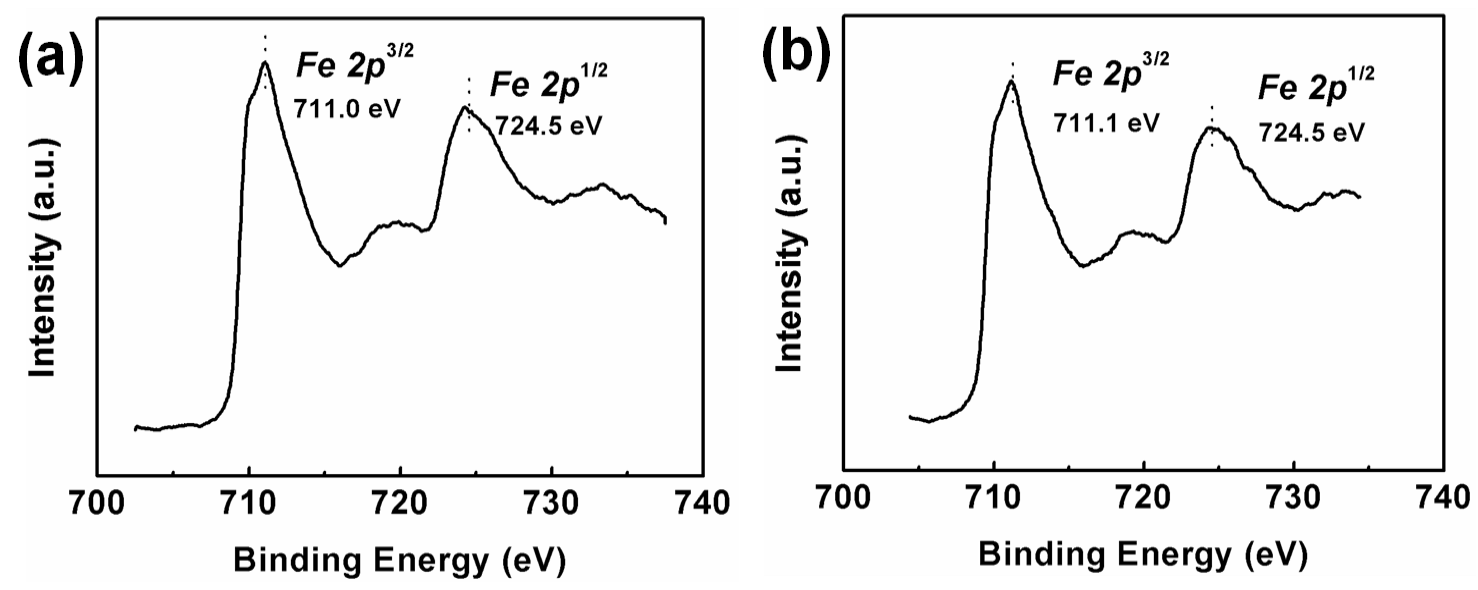

Figure 6-11. XPS spectra of the $\mathrm{Fe} 2 \mathrm{p}$ peaks of (a) $\alpha-\mathrm{Fe}_{2} \mathrm{O}_{3}$ and (b) $\alpha-\mathrm{Fe}_{2} \mathrm{O}_{3} / \mathrm{rGO}$ after heating at $800{ }^{\circ} \mathrm{C}$ for activation.

However, there was no change in the XPS spectra of $\mathrm{Fe} 2 \mathrm{p}$ of the $\alpha-\mathrm{Fe}_{2} \mathrm{O}_{3}$ and the $\alpha-\mathrm{Fe}_{2} \mathrm{O}_{3} / \mathrm{rGO}$ in Supporting Information, Figure 6-11. The characterization data demonstrated that the $\alpha-\mathrm{Fe}_{2} \mathrm{O}_{3} / \mathrm{rGO}$ composite was successfully formed. The specific surface areas were $19.3 \mathrm{~m}^{2} / \mathrm{g}$ and $24.9 \mathrm{~m}^{2} / \mathrm{g}$ for the pure $\alpha-\mathrm{Fe}_{2} \mathrm{O}_{3}$ sample and the $\mathrm{rGO} / \alpha$ $\mathrm{Fe}_{2} \mathrm{O}_{3}$ composite, respectively. 


\subsubsection{Photocatalytic oxygen generation}

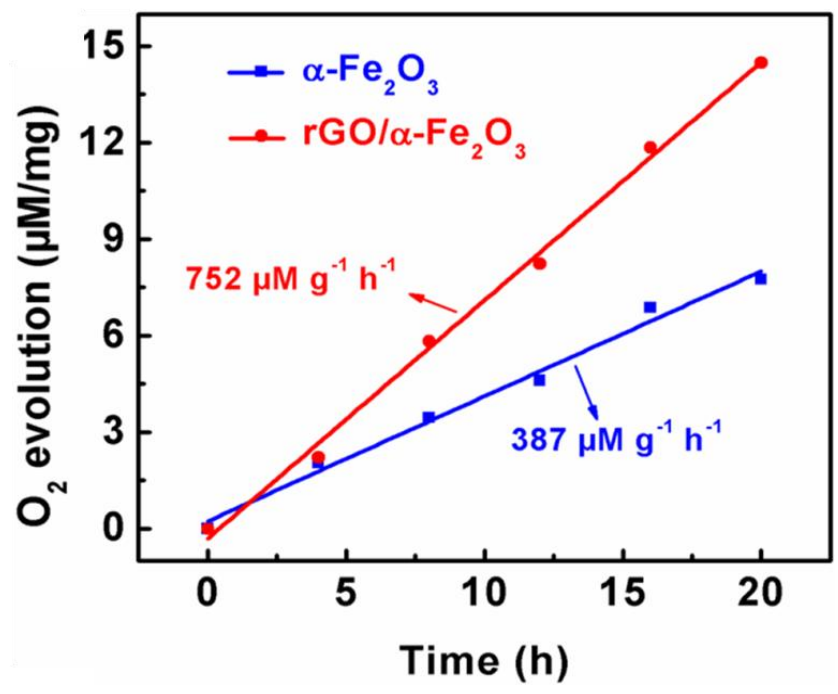

Figure 6-12. Normalized $\mathrm{O}_{2}$ evolution plots of the $\alpha-\mathrm{Fe}_{2} \mathrm{O}_{3}$ and $\alpha-\mathrm{Fe}_{2} \mathrm{O}_{3} / \mathrm{rGO}$

Both the $\alpha-\mathrm{Fe}_{2} \mathrm{O}_{3}$ and the $\alpha-\mathrm{Fe}_{2} \mathrm{O}_{3} / \mathrm{rGO}$ were irradiated under simulated solar light to evaluate the photocatalytic activity toward oxygen evolution from water. As shown in Figure 6-12, the $\mathrm{O}_{2}$ generation rate was measured to be $387 \mu \mathrm{M} / \mathrm{g} / \mathrm{h}$ for the $\alpha-\mathrm{Fe}_{2} \mathrm{O}_{3}$ and $752 \mu \mathrm{M} / \mathrm{g} / \mathrm{h}$ for the $\alpha-\mathrm{Fe}_{2} \mathrm{O}_{3} / \mathrm{rGO}$ composite, respectively. Normalized with surface areas, the $\mathrm{O}_{2}$ generation rates were $20.1 \mu \mathrm{M} / \mathrm{g} / \mathrm{h}$ and $30.2 \mu \mathrm{M} / \mathrm{g} / \mathrm{h}$ for the $\alpha-\mathrm{Fe}_{2} \mathrm{O}_{3}$ and $\alpha$ $\mathrm{Fe}_{2} \mathrm{O}_{3} / \mathrm{rGO}$ composite, respectively. Coupling a semiconductor with $\mathrm{rGO}$ is one of the effective routes to improve the photocatalytic activity of semiconductors $[25,26]$. In the present work, the $\alpha-\mathrm{Fe}_{2} \mathrm{O}_{3} / \mathrm{rGO}$ composites with different rGO contents exhibited difference in the photocatalytic activity toward oxygen evolution. 

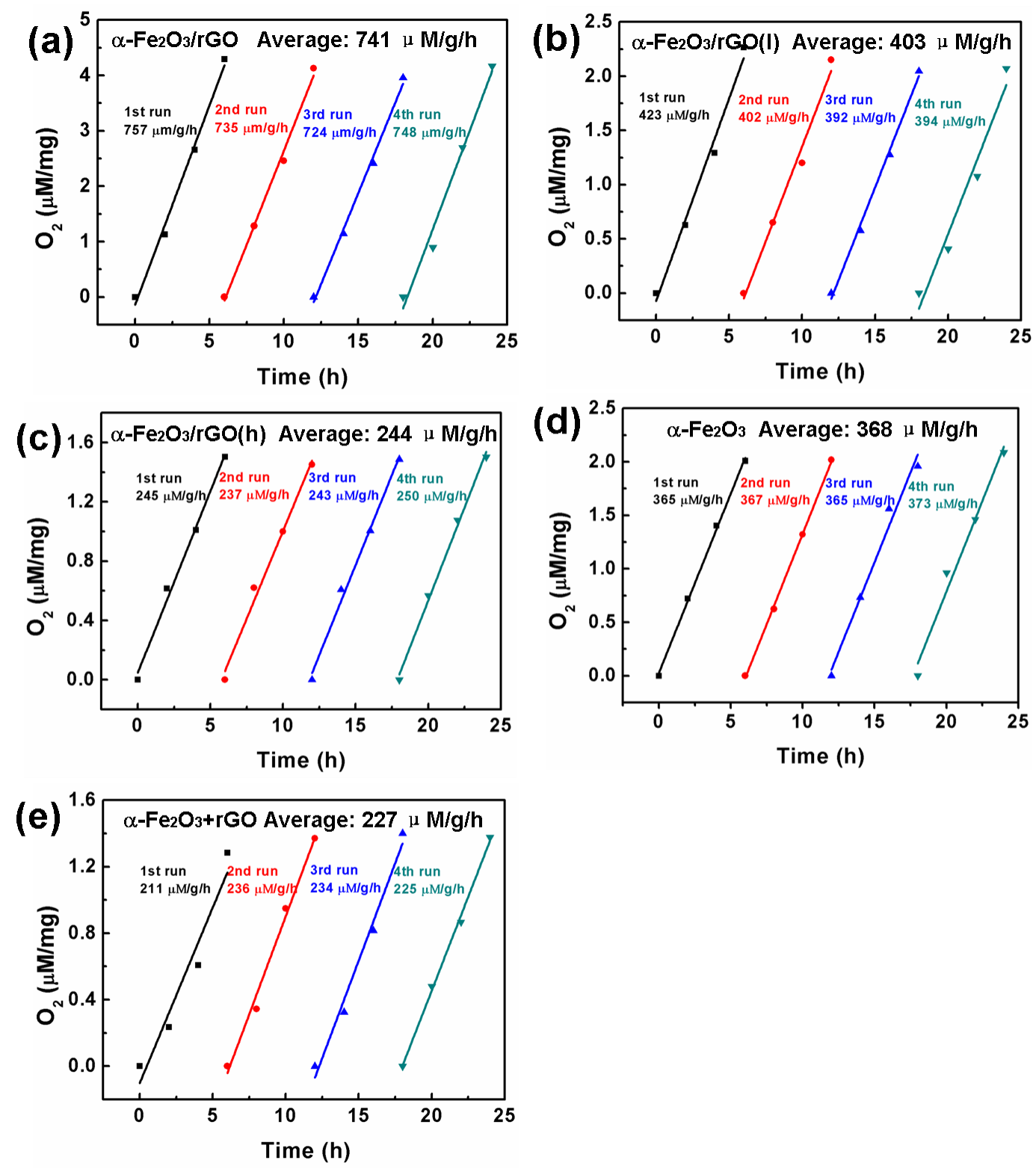

Figure 6-13. Photocatalytic stability measurement (a) $\alpha-\mathrm{Fe}_{2} \mathrm{O}_{3} / \mathrm{rGO}$ containing $3.8 \mathrm{wt} \%$ of rGO, (b) $\alpha$ $\mathrm{Fe}_{2} \mathrm{O}_{3} / \mathrm{rGO}$ (1) containing 2.9 wt $\%$ of $\mathrm{rGO}$, (c) $\alpha-\mathrm{Fe}_{2} \mathrm{O}_{3} / \mathrm{rGO}$ (h) containing $6.1 \mathrm{wt} \%$ of $\mathrm{rGO}$, (d) $\alpha-\mathrm{Fe}_{2} \mathrm{O}_{3}$, and (e) physical mixture of $\alpha-\mathrm{Fe}_{2} \mathrm{O}_{3}+\mathrm{rGO}$ containing $3.8 \mathrm{wt} \%$ of $\mathrm{rGO}$

A too high rGO content resulted in relatively scarce $\alpha-\mathrm{Fe}_{2} \mathrm{O}_{3}$ particles on the rGO sheets (Figure 6-1). A too low rGO content led to the heavily agglomerated $\alpha-\mathrm{Fe}_{2} \mathrm{O}_{3}$ particles on the rGO sheets (Figure 6-3); even some of $\alpha-\mathrm{Fe}_{2} \mathrm{O}_{3}$ particles were not 
supported on the rGO sheets. Consequently, both the $\alpha-\mathrm{Fe}_{2} \mathrm{O}_{3} / \mathrm{rGO}(\mathrm{h})$ (Figure 6-13 (c)) and the $\alpha-\mathrm{Fe}_{2} \mathrm{O}_{3} / \mathrm{rGO}(\mathrm{l})$ (Figure 6-13 (b)) exhibited worse photocatalytic activity than the $\alpha-\mathrm{Fe}_{2} \mathrm{O}_{3} / \mathrm{rGO}$ with an optimal rGO content (Figure 6-13 (a)). In addition, a physical mixture of $\alpha-\mathrm{Fe}_{2} \mathrm{O}_{3}$ with rGO was prepared as a control sample. This physical mixture ( $\alpha$ $\mathrm{Fe}_{2} \mathrm{O}_{3}+\mathrm{rGO}$ ) in Figure 6-13 (e) showed much worse photocatalytic activity than the hydrothermally prepared $\alpha-\mathrm{Fe}_{2} \mathrm{O}_{3} / \mathrm{rGO}$ composite (Figure 6-13 (a)) although both the samples had the same rGO content $(3.8 \mathrm{wt} \%)$. According to the average photocatalytic $\mathrm{O}_{2}$ evolution rate measured with four cycles, the five samples showed the order: $\alpha$ $\mathrm{Fe}_{2} \mathrm{O}_{3} / \mathrm{rGO}>\alpha-\mathrm{Fe}_{2} \mathrm{O}_{3} / \mathrm{rGO}(\mathrm{h})>\alpha-\mathrm{Fe}_{2} \mathrm{O}_{3}>\alpha-\mathrm{Fe}_{2} \mathrm{O}_{3} / \mathrm{rGO}(\mathrm{l})>\alpha-\mathrm{Fe}_{2} \mathrm{O}_{3}+\mathrm{rGO}$. This indicated that the interaction of $\alpha-\mathrm{Fe}_{2} \mathrm{O}_{3}$ with $\mathrm{rGO}$ played an important role in the photocatalytic activity.

\subsubsection{Photoelectrochemical measurements}

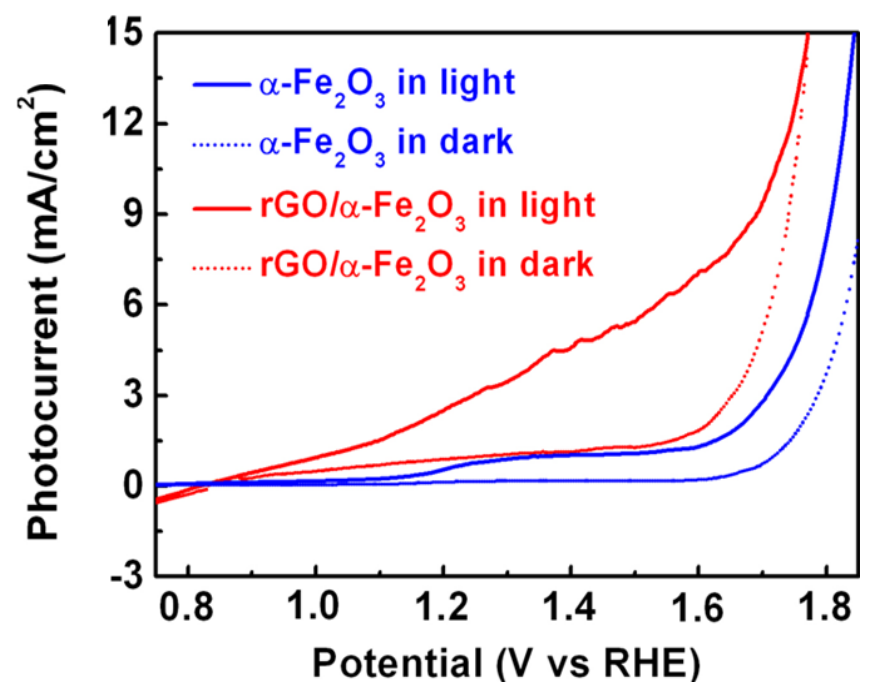

Figure 6-14. Photocurrent densities $(J)$ as a function of the applied voltage (V vs RHE) of the $\alpha-\mathrm{Fe}_{2} \mathrm{O}_{3}$ and $\alpha-\mathrm{Fe}_{2} \mathrm{O}_{3} / \mathrm{rGO}$ with and without irradiation under AM $1.5 \mathrm{G}$ simulated solar light. 
To further understand why the $\alpha-\mathrm{Fe}_{2} \mathrm{O}_{3} / \mathrm{rGO}$ composite showed better photocatalytic activity than $\alpha-\mathrm{Fe}_{2} \mathrm{O}_{3}$ alone, both the powder samples were immobilized on the FTO electrodes. Figure 6-14 shows the PEC behavior of both the samples. The photocurrent observed in $\alpha-\mathrm{Fe}_{2} \mathrm{O}_{3}$ started at $1.05 \mathrm{~V}$ vs $\mathrm{RHE}$, raised to $0.80 \mathrm{~mA} \mathrm{~cm}{ }^{-2}$ at 1.23 $\mathrm{V}$ vs RHE, and finally reached $1.30 \mathrm{~mA} \mathrm{~cm}^{-2}$ before the dark current onset. For the $\alpha-\mathrm{Fe}_{2} \mathrm{O}_{3} / \mathrm{rGO}$, the photocurrent onset shifted to lower potential $(0.8 \mathrm{~V}$ vs $\mathrm{RHE})$ and the photocurrent density increased continuously up to $6 \mathrm{~mA} \mathrm{~cm}^{-2}$ before the dark current onset. Small oscillation of photocurrent was observed at around $1.4 \mathrm{eV}$. Similar phenomenon was also shown in the previous literature $[27,28]$. The dark current for the $\alpha-\mathrm{Fe}_{2} \mathrm{O}_{3} / \mathrm{rGO}$ indicated that some electrons were transferred from rGO to the electrode [29]. Two conclusions were drawn from the $J$ - $V$ curves. First, the lower onset potential of the $\alpha-\mathrm{Fe}_{2} \mathrm{O}_{3} / \mathrm{rGO}$ indicates a smaller kinetic energy barrier for charge transfer across the interface of $\mathrm{rGO}$ and $\alpha-\mathrm{Fe}_{2} \mathrm{O}_{3}$ [4,5]. In other words, $\mathrm{rGO}$ can lower the overpotential required for water oxidation of $\alpha-\mathrm{Fe}_{2} \mathrm{O}_{3}$. Second, the photocurrent of the $\alpha-\mathrm{Fe}_{2} \mathrm{O}_{3} / \mathrm{rGO}$ composite $\left(6 \mathrm{~mA} \cdot \mathrm{cm}^{-2}\right)$ was higher than the pristine $\alpha-\mathrm{Fe}_{2} \mathrm{O}_{3}\left(1.0-3.0 \mathrm{~mA} \cdot \mathrm{cm}^{-2}\right)$, which was in agreement with the previous literature [4,5]. The higher photocurrent of the $\alpha$ $\mathrm{Fe}_{2} \mathrm{O}_{3} / \mathrm{rGO}$ must be due to more electrons being transferred to the photo-electrode, which is only possible if $\mathrm{rGO}$ acted as an electron transfer channel, transferring the photogenerated electrons from $\alpha-\mathrm{Fe}_{2} \mathrm{O}_{3}[29,30]$. 

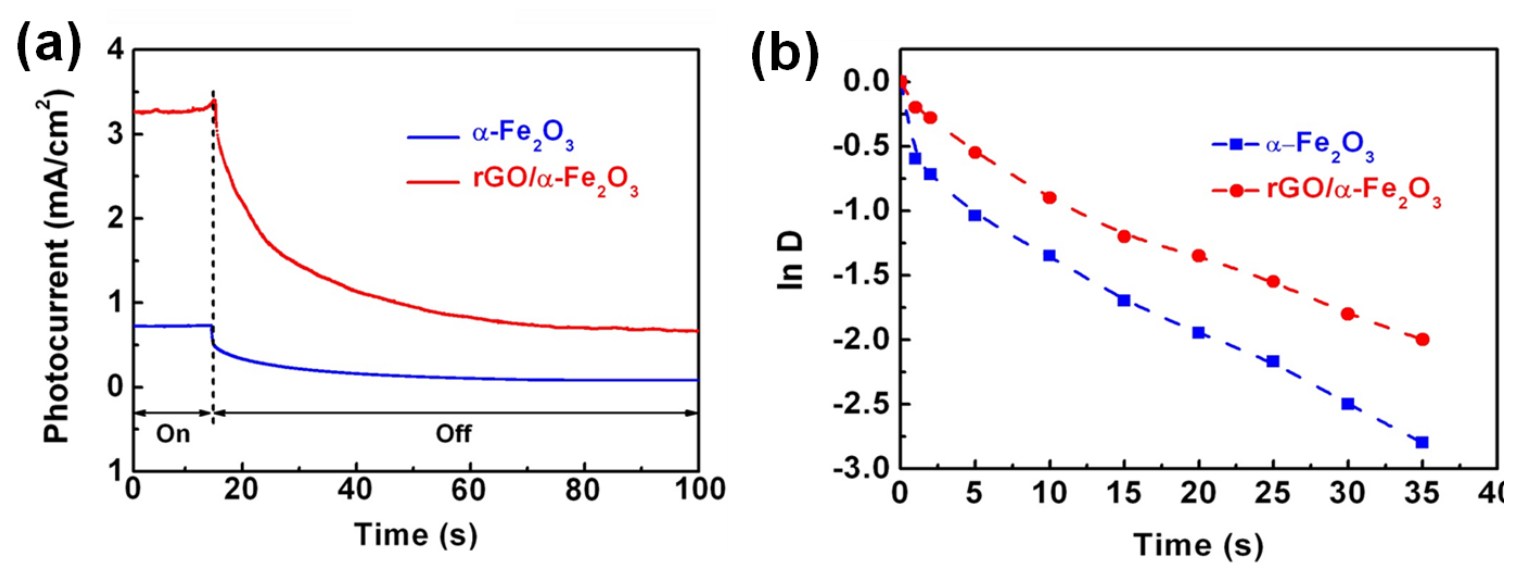

Figure 6-15. (a) Photocurrent decay curves of the $\alpha-\mathrm{Fe}_{2} \mathrm{O}_{3}$ and $\alpha-\mathrm{Fe}_{2} \mathrm{O}_{3} / \mathrm{rGO}$ at the bias of $1.23 \mathrm{~V}$ vs RHE;

(b) Normalized plots of the photocurrent-time dependence for the $\alpha-\mathrm{Fe}_{2} \mathrm{O}_{3}$ and $\alpha-\mathrm{Fe}_{2} \mathrm{O}_{3} / \mathrm{rGO}$.

Electron transfer to the rGO could increase the charge separation and suppress the charge recombination, leaving long lived holes in the $\alpha-\mathrm{Fe}_{2} \mathrm{O}_{3}$ to oxidize water, increasing the photocatalytic water splitting rate. Transient photocurrent plots for $\alpha-\mathrm{Fe}_{2} \mathrm{O}_{3}$ and $\alpha-\mathrm{Fe}_{2} \mathrm{O}_{3} / \mathrm{rGO}$ photo-electrodes at a constant potential (1.23 V vs RHE) confirmed this scenario. After the potential was removed, the $\alpha-\mathrm{Fe}_{2} \mathrm{O}_{3}$ revealed a sharper photocurrent drop off than the $\alpha-\mathrm{Fe}_{2} \mathrm{O}_{3} / \mathrm{rGO}$. The photocurrent of the $\mathrm{rGO} / \alpha-\mathrm{Fe}_{2} \mathrm{O}_{3}$ decayed to the dark current level slower than $\alpha-\mathrm{Fe}_{2} \mathrm{O}_{3}$. To quantitatively determine the charge recombination behavior, a normalized parameter $D$ is introduced in Equation 6-2: [31]

$$
D=\left(I_{t}-I_{s t}\right) /\left(I_{\text {in }}-I_{s t}\right)
$$

where $I_{t}, I_{s t}$, and $I_{i n}$ are the time-dependent, steady-state and initial photocurrents, respectively. Figure 6-15 (b) shows the normalized plots of $\ln D^{-} t$. The transient time constant $(\tau)$ is defined as the time when $\ln D=-1$ [31], which reflects the general behavior of charge recombination. From Figure 6-15 (b), $\tau$ was estimated to be $4.8 \mathrm{~s}$ for $\alpha-\mathrm{Fe}_{2} \mathrm{O}_{3}$ 
and $10.6 \mathrm{~s}$ for $\alpha-\mathrm{Fe}_{2} \mathrm{O}_{3} / \mathrm{rGO}$. The larger transient time constant for the composite structure indicates that it has a lower charge recombination rate than the $\alpha-\mathrm{Fe}_{2} \mathrm{O}_{3}$ alone.

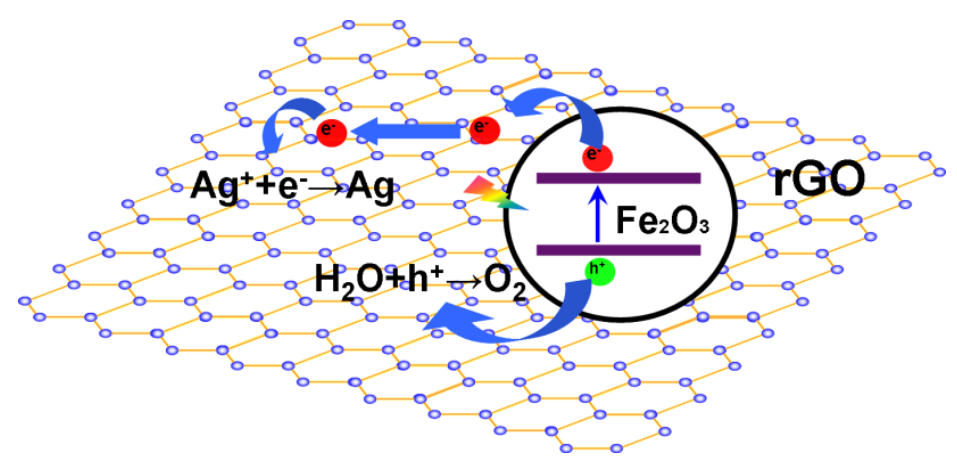

Figure 6-16. Schematic of charge transfer in the $\alpha-\mathrm{Fe}_{2} \mathrm{O}_{3} / \mathrm{rGO}$ composite.

Transient absorption measurements proved the photocatalysis enhancement mechanism in the $\alpha-\mathrm{Fe}_{2} \mathrm{O}_{3} / \mathrm{rGO}$ composite as illustrated in Figure 6-16. In this scheme, mobile electrons are initially created in $\alpha-\mathrm{Fe}_{2} \mathrm{O}_{3}$, quickly transfer to the $\mathrm{rGO}$ sheet, where they diffuse into trap states in the rGO on a time scale of picoseconds. These long-lived trapped electrons then react with the $\mathrm{Ag}^{+}$, while the accumulated holes in the $\alpha-\mathrm{Fe}_{2} \mathrm{O}_{3}$ lead to water oxidation, generating $\mathrm{O}_{2}$. Alternatively, in a PEC, the trapped electrons can hop between successive layers of rGO to reach the photoelectrode, leading to an increase in the photocurrent and the recombination time. The interface of $\mathrm{rGO}$ and $\alpha-\mathrm{Fe}_{2} \mathrm{O}_{3}$ enhances the charge separation rate, increasing the photocurrent.

\subsection{Conclusions}

In summary, an $\alpha-\mathrm{Fe}_{2} \mathrm{O}_{3} / \mathrm{rGO}$ composite was synthesized for photocatalytic water oxidation. The suppressed charge recombination and enhanced charge separation in $\alpha$ - 
$\mathrm{Fe}_{2} \mathrm{O}_{3}$ was realized due to the extraction of photogenerated electrons from $\alpha-\mathrm{Fe}_{2} \mathrm{O}_{3}$ to rGO, which resulted in the enhanced photocatalytic activity toward oxygen evolution. This mechanistic study has paved a way for improving the photocatalytic activity of semiconductors with the poor charge mobility and the short charge carrier diffusion length.

\section{Reference}

[1] Tilley, S. D., Cornuz, M., Sivula, K., Gratzel, M., Angew. Chem. Int. Ed., 2010, 49, 6405.

[2] Sivula, K., Zboril, R., Formal, F. L., Robert, R., Weidenkaff, A., Tucek, J., Frydrych, J., Gratzel, M., J. Am. Chem. Soc., 2010, 132, 7436.

[3] Li, L., Yu, Y. H., Meng, F., Tan, Y. Z., Hamers, R. J., Jin, S., Nano Lett., 2012, 12, 724.

[4] Wang, G. M., Ling, Y. C., Wheeler, D. A., George, K. N., Horsley, K., Heske, C., Zhang, J. Z., Li, Y., Nano Lett., 2011, 11, 3503.

[5] Townsend, T. K., Sabio, E. M., Browning, N. D., Osterloh, F. E., Energy Environ. Sci., 2011, 4, 4270.

[6] Kay, A., Cesar, I., Gratzel, M., J. Am. Chem. Soc., 2006, 128, 15714.

[7] Cesar, I., Kay, A., Martinez, J. A. G., Gratzel, M., J. Am. Chem. Soc., 2006, 128, 4582.

[8] Khan, S. U. M., Akikusa, J., J. Phys. Chem. B, 1999, 103, 7184.

[9] Duret, A., Gratzel, M., J. Phys. Chem. B, 2005, 109, 17184.

[10] Goncalves, R. H., Lima, B. H. R., Leite, E. R., J. Am. Chem. Soc., 2011, 133, 6012. 
[11] Meng, F. K., Hong, Z. L., Arndt, J., Li, M., Zhi, M. J., Yang, F., Wu, N. Q., Nano Res., 2012, 5, 213.

[12] Wang, J., Tafen, D. N., Lewis, J. P., Hong, Z. L., Manivannan, A., Zhi, M. J., Li, M., Wu, N. Q., J. Am. Chem. Soc., 2009, 131, 12290.

[13] Wu, N. Q., Wang, J., Tafen, D. N., Wang, H., Zheng, J. G., Lewis, J. P., Liu, X. G., Leonard, S. S., Manivannan, A., J. Am. Chem. Soc., 2010, 132, 6679.

[14] Meng, F. K., Li, J. T., Hong, Z. L., Zhi, M. J., Sakla, A., Xiang, C. C., Wu, N. Q., Catal. Today, 2012, j. cattod. 2012.05.038.

[15] Zhong, D. K., Sun, J. W., Inumaru, H., Gamelin, D. R., J. Am. Chem. Soc., 2009, $131,6086$.

[16] Li, J. T., Meng, F. K., Suri, S., Ding, W. Q., Huang, F. Q., Wu, N. Q., Chem. Commun., 2012, 10.1039/C2CC30376K.

[17] Lightcap, I. V., Kosel, T. H., Kamat, P. V., Nano Lett., 2010, 10, 577.

[18] Li, N., Liu, G., Zhen, C., Li, F., Zhang, L. L., Cheng, H. M., Adv. Funct. Mater., 2011, 21, 1717.

[19] Wu, N. Q., Fu, L., Su, M., Aslam, M., Wong, K. C., Dravid, V. P., Nano Lett., 2004, $4,383$.

[20] Lim, M. S., Feng, K., Chen, X. Q., Wu, N. Q., Raman, A., Nightingale, J., Gawalt, E. S., Korakakis, D., Hornak, L. A., Timperman, A. T., Langmuir, 2007, 23, 2444.

[21] Stankovich, S., Piner, R. D., Chen, X. Q,, Wu, N. Q., Nguyen, S. T., Ruoff, R. S., J. Mater. Chem., 2006, 16, 155.

[22] Ng, Y. H., Iwase, A., Kudo, A., Amal, R., J. Phys. Chem. Lett., 2010, 1, 2607. 
[23] Jia, L., Wang, D. H., Huang, Y. X., Xu, A. W., Yu, H. Q., J. Phys. Chem. C., 2011, $115,11466$.

[24] Hagfeldt, A., Lindstrom, H., Sodergren, S., Lindquist, S. E., J. Electroanal. Chem., 1995, 381, 39.

[25] Tang, L. H., Wang, Y., Li, Y. M., Feng, H. B., Lu, J., Li, J. H., Adv. Funct. Mater., 2009, 19, 2782.

[26] Ulbricht, R., Hendry, E., Shan, J., Heinz, T. F., Rev. Mod. Phys., 2011, 3, 543.

[27] Kaniyankandy, S., Achary, S. N., Rawalekar, S., Ghosh, H. N., J. Phys. Chem. C, 2011, 115, 19110.

[28] Chang, C. Y.; Wang, C. H.; Tseng, C. J.; Cheng, K. W.; Hourng, L. W.; Tsai, B. T. Int. J. Hydrogen Energy 2012, 37, 13616.

[29] Ng, Y. H.; Iwase, A.; Kudo, A.; Amal, R. J. Phys. Chem. Lett. 2010, 1, 2607.

[30] Jia, L.; Wang, D. H.; Huang, Y. X.; Xu, A. W.; Yu, H. Q. J. Phys. Chem. C 2011, $115,11466$.

[31] Tafalla, D.; Salvador, P.; Benito, R. M. J. Electrochem. Soc. 1990, 137, 1810. 


\section{Chapter 7 Solar $\mathrm{H}_{2}$ Generation of $\mathrm{Au} / \mathrm{La}_{2} \mathrm{Ti}_{2} \mathrm{O}_{7} / \mathbf{r G O}$}

\section{Heterostructure Enhancement by Plasmonic Energy Transfer}

\subsection{Background and introduction}

Hydrogen generation by photocatalytic water splitting is a promising technique to provide clean solar fuel to meet the sustainable development needs of the world [1-4]. For single material devices a trade off exists between large band gap oxides with good carrier recombination and surface kinetics but poor solar utilization, and small band gap materials with good spectral utilization but poor charge recombination and stability [515]. Doping is one successful approach to increasing spectral utilization in large band gap oxides [12,16-18]. Unfortunately, the mid gap states created by the dopants increase charge recombination, decreasing the overall photocatalytic performance. This loss can be avoided if the dopant forms a continuum with the valence or conduction band edge, preventing localized states mid gap as shown in Chapters 3-5. Unfortunately, the requirement for a connected density of states ultimately limits the extent to which the band gap can be narrowed $[16,18]$. As well, in Chapter 6 we have shown the effects of using rGO as a charge extraction layer. Although LTO has good carrier mobility compared to $\mathrm{Fe}_{2} \mathrm{O}_{3}$, a large enhancement in performance can still be seen by improving carrier recombination lifetimes.

Therefore, in this chapter we combine the results of the enhancements found in Chapters 3-6 with a different avenue to increasing the photocatalytic performance, plasmonics. Plasmonics can lead to enhancements in both charge separation [19-21] and 
carrier lifetime [22-24]. First, plasmonics can increase spectral utilization at energies below the band edge through plasmon induced resonant energy transfer (PIRET) and direct electron transfer (DET), or increase absorption at wavelengths above the band edge by increased scattering [19-21]. PIRET is a nonradiative energy transfer process, so its strength depends on the spectral overlap between the plasmon and semiconductor. The strength of DET depends on the ability of photo excited plasmonic electrons to overcome the Schottky barrier present at the metal/semiconductor interface [20].

Also, the plasmonic metal nanoparticle can increase carrier lifetime and the reduction potential by the Fermi level shift at the semiconductor/metal/electrolyte interface [22-24]. Au nanoparticles have also been shown to act as an electron relay in heterostructures, further increasing charge separation [25]. Plasmonics can therefore further increase the spectral utilization of a doped semiconductor while also decreasing the negative effect on carrier lifetimes. Despite this possibility, little research has investigated the interaction of dopants with plasmonics.

Our previous work has shown that Pt-loaded $\mathrm{La}_{2} \mathrm{Ti}_{2} \mathrm{O}_{7}$ (Pt-LTO) can be doped with nitrogen to form a continuum of states above the band edge, extending the absorption into the visible without negatively effecting carrier lifetimes [16]. However, the $\mathrm{N}$-doped LTO still has a band gap below the ideal $2 \mathrm{eV}$ value and LTO is primarily an ionic conductor, limiting charge migration [7,16,32-35].

We have shown that by combining the individual enhancement avenues of doping and plasmonics, the hydrogen generation efficiency of nitrogen doped LTO can be increased from 65.3 to $163.4 \mu \mathrm{M} \cdot \mathrm{g}^{-1} \cdot \mathrm{h}^{-1}$, beyond the sum of each individual enhancement. 


\subsection{Experimental section}

\subsubsection{Materials synthesis}

Pt-loaded $\mathrm{La}_{2} \mathrm{Ti}_{2} \mathrm{O}_{7}$ nanosheets were synthesized as a precursor material. The detail synthesis process was described as follows. First, $\mathrm{La}_{2} \mathrm{Ti}_{2} \mathrm{O}_{7}$ nanosheets were synthesized as the procedures in chapter 4 and 5. Second, $0.05 \mathrm{~g}$ of $\mathrm{Pt}\left(\mathrm{NH}_{3}\right) 4 \mathrm{Cl}_{2}(\mathrm{Sigma}-$ Aldrich) and $0.5 \mathrm{~g}$ powder of $\mathrm{La}_{2} \mathrm{Ti}_{2} \mathrm{O}_{7}$ nanosheets were mixed in $20 \mathrm{~mL}$ of deionized water (DI) to form a homogenous suspension. Third, the suspension was stirred rigorously for $24 \mathrm{hrs}$ at room temperature. Fourth, the suspension was centrifuged to obtain a fluffy white powder, which was then dried at $80{ }^{\circ} \mathrm{C}$ overnight. Fifth, the dried white powder was heated in air at $400{ }^{\circ} \mathrm{C}$ for $3 \mathrm{hrs}$ to become a grey powder. Sixth, the grey powder was reduced at $500{ }^{\circ} \mathrm{C}$ in a $\mathrm{H}_{2}$ flow for $1 \mathrm{hr}$. Consequently, four samples were synthesized based the precursor of Pt-loaded $\mathrm{La}_{2} \mathrm{Ti}_{2} \mathrm{O}_{7}$ nanosheets:

1.1 Au,Pt-loaded $\mathrm{La}_{2} \mathrm{Ti}_{2} \mathrm{O}_{7}$ nanosheets coupled with rGO was synthesized as follows: first, mixed $0.5 \mathrm{~g}$ powder of Pt-loaded $\mathrm{La}_{2} \mathrm{Ti}_{2} \mathrm{O}_{7}$ nanosheets with $0.05 \mathrm{~g}$ of $\mathrm{HAuCl}_{4}$ (Alfa. Inc) in $10 \mathrm{~mL}$ DI water to form a suspension; second, centrifuged the suspension to get a fluffy powder and then dry the powder at $80{ }^{\circ} \mathrm{C}$ overnight; third, heated the powder in air at $400{ }^{\circ} \mathrm{C}$ for $3 \mathrm{hrs}$; fourth, reduced the powder in a $\mathrm{H}_{2}$ flow at $500{ }^{\circ} \mathrm{C}$ for $1 \mathrm{hr}$; fifth, synthesized graphene oxide (GO) as the Hummer method; sixth, mixed 0.5 g powder of the Au,Pt-loaded $\mathrm{La}_{2} \mathrm{Ti}_{2} \mathrm{O}_{7}$ nanosheets with $0.0125 \mathrm{~g}$ of $\mathrm{GO}(2.5$ wt.\%) in $15 \mathrm{~g}$ of DI water to make a suspension; seventh, stirred the suspension rigorously for $20 \mathrm{sec}$ in ultrasonic bath; eighth, added $15 \mathrm{ml}$ of ethanol in the suspension and then stirred it for 1 
min; ninth, sealed the suspension in a teflon-lined pressure vessel, which was then placed in a pre-heated oven $\left(200{ }^{\circ} \mathrm{C}\right)$ for $24 \mathrm{hrs}$; tenth, cooled the pressure vessel to room temperature and washed the sample 5-6 times with DI water; eleventh, dried the sample at $80{ }^{\circ} \mathrm{C}$ on a hotplate overnight.

1.2 Pt-loaded, $\mathrm{N}$-doped $\mathrm{La}_{2} \mathrm{Ti}_{2} \mathrm{O}_{7}$ nanosheets was synthesized as follows: heated $0.5 \mathrm{~g}$ powder of Pt-loaded $\mathrm{La}_{2} \mathrm{Ti}_{2} \mathrm{O}_{7}$ nanosheets in a $\mathrm{NH}_{3}$ flow at $600{ }^{\circ} \mathrm{C}$ for $3 \mathrm{hrs}$ with a ramp rate of $.5^{\circ} \mathrm{C} / \mathrm{min}$ in a tubular furnace.

1.3 Au,Pt-loaded, $\mathrm{N}$-doped $\mathrm{La}_{2} \mathrm{Ti}_{2} \mathrm{O}_{7}$ nanosheets was synthesized as follows: heated $0.5 \mathrm{~g}$ powder of Au,Pt-loaded $\mathrm{La}_{2} \mathrm{Ti}_{2} \mathrm{O}_{7}$ nanosheets in a $\mathrm{NH}_{3}$ flow at $600{ }^{\circ} \mathrm{C}$ for $3 \mathrm{hrs}$ with a ramp rate of $.5^{\circ} \mathrm{C} / \mathrm{min}$ in a tubular furnace.

1.4 Au,Pt-loaded, N-doped $\mathrm{La}_{2} \mathrm{Ti}_{2} \mathrm{O}_{7}$ nanosheets coupled with $\mathrm{rGO}$ was synthesized with hydrothermal method by mixing $0.5 \mathrm{~g}$ powder of Au,Pt-loaded, N-doped $\mathrm{La}_{2} \mathrm{Ti}_{2} \mathrm{O}_{7}$ nanosheets and GO as described in 1.1.

Finally, the four samples of $\mathrm{Au}$,Pt-loaded $\mathrm{La}_{2} \mathrm{Ti}_{2} \mathrm{O}_{7}$ nanosheets coupled with $\mathrm{rGO}$, Pt-loaded, N-doped $\mathrm{La}_{2} \mathrm{Ti}_{2} \mathrm{O}_{7}$ nanosheets, Au,Pt-loaded, N-doped $\mathrm{La}_{2} \mathrm{Ti}_{2} \mathrm{O}_{7}$ nanosheets and $\mathrm{Au}$,Pt-loaded, $\mathrm{N}$-doped $\mathrm{La}_{2} \mathrm{Ti}_{2} \mathrm{O}_{7}$ nanosheets coupled with rGO were collected and named shortly as Au@Pt-LTO/rGO, Pt-NLTO, Au@Pt-NLTO and Au@Pt-NLTO/rGO respectively. 


\subsubsection{Materials characterization}

The detail materials characterization methods were reported in chapter 4 and 5 . Briefly, the morphology was characterized with a scanning electron microscope (SEM, JEOL 7600F) and a transmission electron microscope (TEM, JEOL, JEM 2100). The crystal structures of the materials were characterized by a high-resolution TEM (HRTEM) and X-ray diffraction (XRD, X' Pert Pro PW3040-Pro, Panalytical Inc.) with Cu Ka radiation. X-ray photoelectron spectroscopy (XPS, PHI 5000 Versa Probe system, Physical Electronics) was used for element (N, Au and Pt) composition analysis with a reference to the $\mathrm{C} 1 \mathrm{~s}$ peak at $284.8 \mathrm{eV}$. The light absorption performance of the samples were characterized by an ultraviolet-visible spectrometer (UV-Vis, Lambda 750, PerkinElmer Inc.). The surface areas of the samples were measured by Brunauer, Emmett and Teller (BET, Micromeritics ASAP 2020).

\subsubsection{Photocatalysis}

The light source used for photocatalytic $\mathrm{H}_{2}$ generation was a commercial solar light simulator (300 W, Newport, Intensity: 40-50 mW/cm ${ }^{2}$, which was equipped with monochromic shutter and an AM 1.5G filter. Briefly, $10 \mathrm{mg}$ powder of the sample was dissolved in $20 \mathrm{~mL}$ of solution $(10 \mathrm{~mL}$ of $\mathrm{DI}$ and $10 \mathrm{~mL}$ of ethanol); then the solution was transferred into a $100 \mathrm{~mL}$ of quart flask and bubbled with $\mathrm{N}_{2}(99.9 \%$, Air Gas Inc.) for $10 \mathrm{~min}$; after bubbling, the flask was sealed and irradiated under the simulated solar light with a light window of $2.0 \mathrm{~cm}^{2}$ for different times; finally, $5 \mathrm{~mL}$ of the gas sample was collected from the flask for chromatography (GC, Shimadzu 2014) measurement. 


\subsubsection{Photoelectrochemical measurements}

\section{(1) Preparation of photoeletrodes}

Typically, $0.1 \mathrm{~g}$ of the powder sample was stirred with $0.5 \mathrm{~mL}$ of terpineol $(\sim 95 \%$, Sigma-Aldrich) with a magnetic stirrer overnight to form a homogenous sticky suspension; then the sticky suspension was coated onto a piece of fluorine-doped tin oxide (FTO) substrate (Hartford, TEC 15) with doctor blading method to make sure the sample suspension was homogenously coated; after drying the sample-coated FTO photoelectrodes on a hot plate in air, the electrodes were heated in $\mathrm{N}_{2}$ at $550{ }^{\circ} \mathrm{C}$ for $2 \mathrm{hr}$ to ensure strong connection between the sample and the FTO glass substrate; finally, a copper wire was connected with the FTO substrate with silver colloid paste and the sealed with epoxy glue.

\section{(2) Electrochemical measurement}

A Photoelectrochemical cell (PEC) was constructed as a three-electrode system and measured with a Gamry electrochemical station (Reference $3000^{\mathrm{TM}}$ ). The Pt plate, $\mathrm{Ag} / \mathrm{AgCl}$ and sample electrodes were acted as counter electrode, reference electrode and working electrode respectively. The three electrodes were immersed into a $1 \mathrm{M} \mathrm{NaOH}$ (>98.0\%, Sigma-Aldrich) aqueous electrolyte ( $\mathrm{pH}=13.6$, bubbled in pure $\mathrm{N}_{2}$ for $30 \mathrm{~min}$ )

simultaneously for electrochemical testing. The light source for the PEC was the same as that used in photocatalytic water splitting. 
The relationship between current density ( $\mathrm{J})$ and potential (V) was obtained. The potential (vs. $\mathrm{Ag} / \mathrm{AgCl}$ ) was converted to the reversible hydrogen electrode (RHE) according to the Nernst equation:

$$
E_{R H E}=E+0.05916 p H+E_{0}
$$

In the equation 7-1, the $E_{R H E}$ is the potential $v s$. RHE, $E_{0}=0.1976 \mathrm{~V}$ at $25{ }^{\circ} \mathrm{C}$, and $E$ is the measured potential vs. $\mathrm{Ag} \mid \mathrm{AgCl}$. The on-off $J-V$ curves were obtained under a bias of $0.25 \mathrm{~V} v s$. RHE.

The incident photo-to-electron conversion efficiency (IPCE) plots were calculated based on the photocurrents at different incident light wavelengths under a bias of $-0.25 \mathrm{~V}$ vs. RHE. The IPCE equation was as follows:

$$
I P C E=1240 J / \lambda I_{\text {light }}
$$

In equation 7-2, the $\mathrm{J}$ and $I_{\text {light }}$ are measured as the photocurrent and light intensity respectively at a wavelength $\lambda$. $\lambda$ was varied from $250 \mathrm{~nm}$ to $700 \mathrm{~nm}$ with an interval of $25 \mathrm{~nm}$. 


\subsection{Results and discussion}
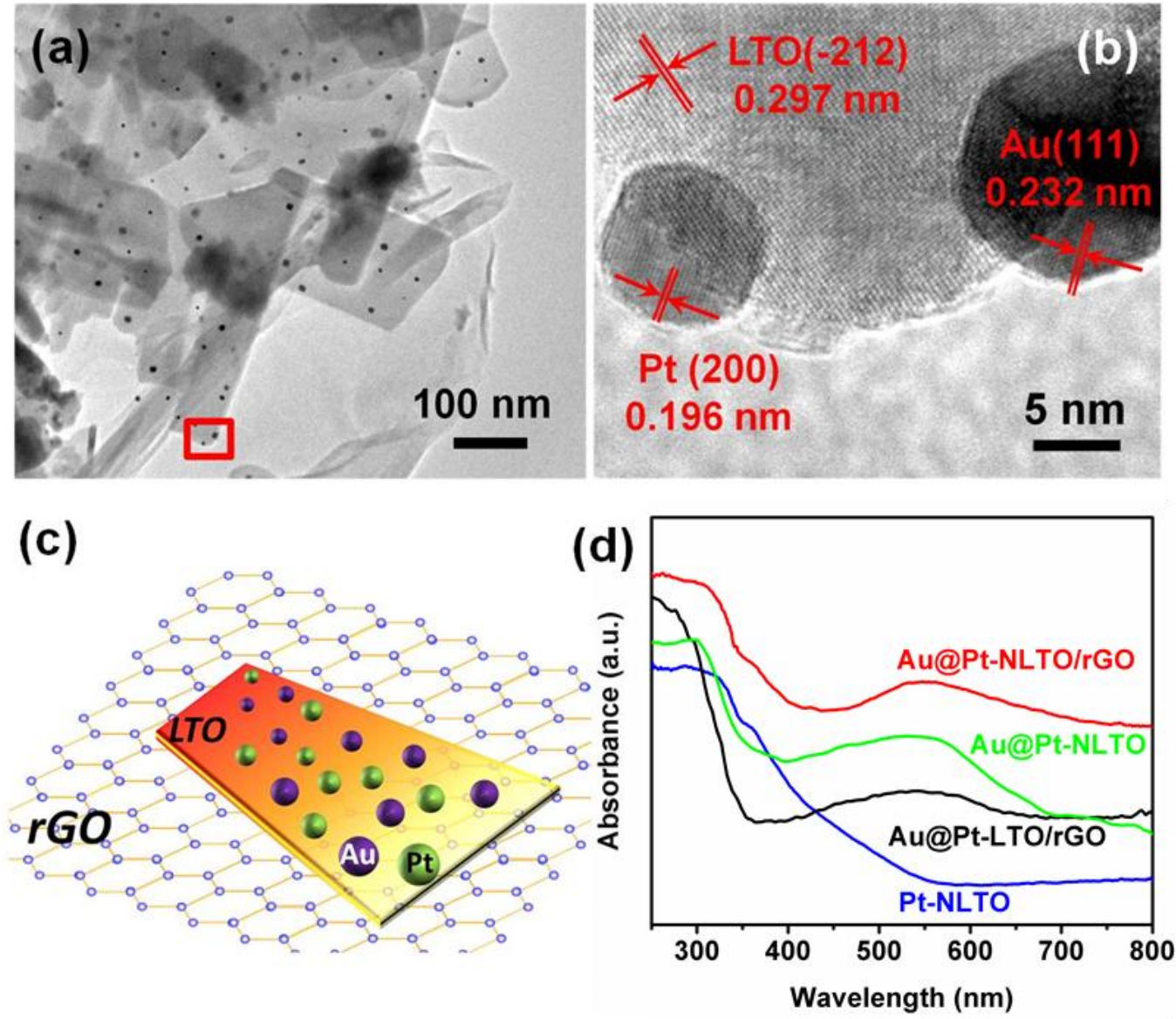

Figure 7-1 Characterization of N-LTO plasmonic and heterostructured samples. The TEM (a) and HRTEM (b) confirmed the presence of Pt and Au nanoparticles on the NLTO/rGO heterostructure, as represented in (c). (d) The band gap of the LTO increased to $550 \mathrm{~nm}$ after doping, with the LSPR of the Au nanoparticles being centered at $550 \mathrm{~nm}$. The addition of rGO led to an increase in absorption across the spectrum.

The NLTO nanosheets with size 200-300 nm were first synthesized with Au or Pt nanoparticles decorating the surface, then combined with rGO in Figure 7-1 (c) $[7,16]$. The TEM and HRTEM images of the Au@Pt-NLTO/rGO are shown in Figure 7-1 (a) and (b). The HRTEM image in Figure 7-1 (b), taken from the red box area in Figure 7-1 
(a), clearly showed that both $\mathrm{Pt}$ and $\mathrm{Au}$ nanoparticle were loaded onto the NLTO nanosheet. The NLTO nanosheet showed a crystal lattice fringe of $0.297 \mathrm{~nm}$, which is attributed to the LTO (-212) facet (JCPDS:28-0517). The left nanoparticle in Figure 7-1 (b) is a Pt (200) facet with a crystal lattice fringe of $0.196 \mathrm{~nm}$ (JCPDS: 04-0802). The right nanoparticle in Figure $1 \mathrm{~b}$ has a lattice fringe of $0.232 \mathrm{~nm}$, which is the $\mathrm{Au}(111)$ facet (JCPDS: 04-0784).
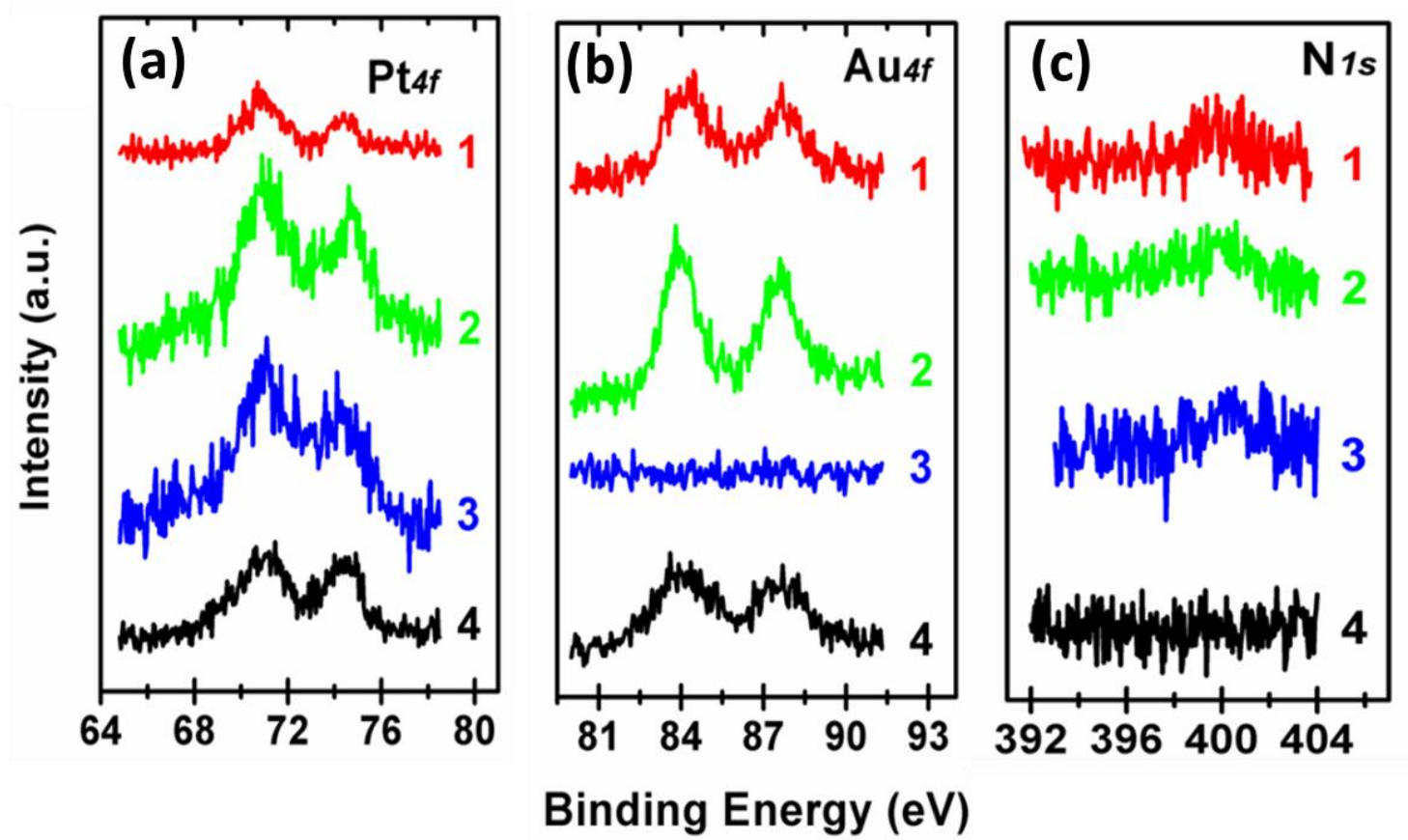

Figure 7-2. XPS of the (a) Pt, (b) Au, and (c) N peaks for Au@Pt-NLTO/rGO (curve 1), Au@Pt-NLTO (curve 2), Pt-NLTO (curve 3), and Au@Pt-LTO/rGO (curve 4). 
Table 7-1. The contents of the loaded Pt and Au and doped $\mathrm{N}$ in the four samples.

\begin{tabular}{|c|c|c|c|}
\hline Sample & Pt (at. \%) & Au (at. \%) & N (at. \%) \\
\hline Au@NLTO/rGO & 1.55 & 2.12 & 1.99 \\
\hline Au@ NLTO & 1.71 & 2.21 & 2.08 \\
\hline NLTO & 1.65 & 0 & 2.13 \\
\hline Au@LTO/rGO & 1.61 & 2.05 & 0 \\
\hline
\end{tabular}

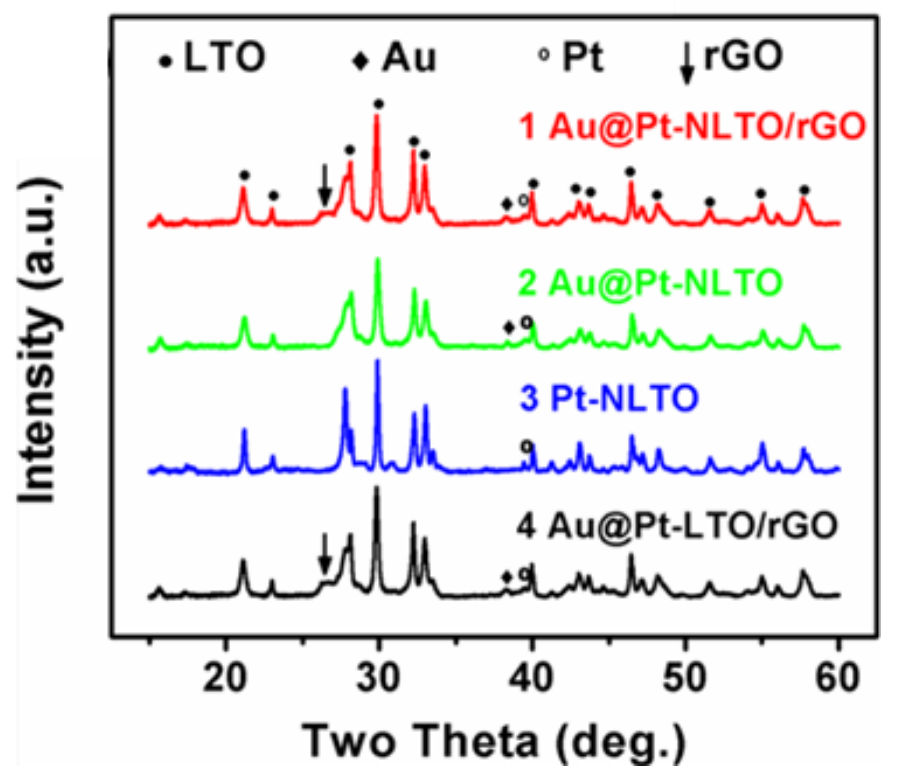

Figure 7-3 XRD and peak classification for Au@Pt-NLTO/rGO (curve 1), Au@Pt-NLTO (curve 2), PtNLTO (curve 3), and Au@Pt-LTO/rGO (curve 4).

The XPS results are in Figure 7-2. Each of the four samples showed a Pt4f peak with two typical sub-peaks of $\mathrm{Pt} 4 \mathrm{f}_{5 / 2}$ and $\mathrm{Pt} 4 \mathrm{f}_{7 / 2}$ at $74.1 \mathrm{eV}$ and $71.0 \mathrm{eV}$ respectively. Except for the Pt-NLTO, the other three samples showed clear Au 4f peaks, which were split into $\mathrm{Au} 4 \mathrm{f}_{5 / 2}$ and $\mathrm{Au} 4 \mathrm{f}_{7 / 2}$ at 87.8 and $83.9 \mathrm{eV}$, respectively. For the three samples 
doped with nitrogen, the $\mathrm{N} 1$ s peak at $398 \mathrm{eV}$ showed a broad peak, which resulted from three typical $\mathrm{N}$ status in the LTO nanosheets $[16,36]$. According to XPS data, the contents of the N, Pt and Au in the samples were calculated (table 7-1). The XRD patterns of the four samples in Figure 7-3 are consistent with the standard XRD data (JCPDS:28-0517) of LTO, which means that the crystal structure of the LTO nanosheets were not changed after nitrogen doping, the Pt or Au loading, or the coupling with GO. Only the Au@Pt-NLTO/rGO and Au@Pt-LTO/rGO showed clear rGO peaks at $27^{\circ}[9$, 37]. A small XRD peak for Au (111) existed at $38.2^{\circ}$ in the XRD patterns of the samples which were loaded with Au nanoparticles. The Pt (111) peak was consistent in all four samples. From the TEM, XPS and XRD data, both the Au and Pt in the samples existed in metallic status instead of oxides on the LTO nanosheets, which indicated the Au and Pt nanoparticles were successfully maintained after nitrogen doping and hydrothermal synthesis.

The absorption data for the four samples is shown in Figure 7-1 (d). The Au@Pt$\mathrm{LTO} / \mathrm{rGO}$ shows the $350 \mathrm{~nm}$, or $3.54 \mathrm{eV}$, band gap of LTO as well as the absorption due to the localized surface plasmon resonance (LSPR) of the Au nanoparticle centered at $550 \mathrm{~nm}$. After nitrogen doping the band gap of LTO decreases to $550 \mathrm{~nm}$, or $2.25 \mathrm{eV}$ $[7,16]$. It should be noted that in LTO the nitrogen doping does not form localized states but instead forms a continuum with the band edge, as discussed in detail in our previous literature [16]. All three samples with Au nanoparticles showed an absorption peak centered at $550 \mathrm{~nm}$ due to the LSPR [20,38,39]. The rGO absorption adds a decreasing offset with wavelength $[9,12]$. The LSPR peak overlaps with the doped N-LTO but not the undoped LTO. Since plasmon induced resonant energy transfer (PIRET) depends on 
the spectral overlap between the semiconductor and plasmon, this spectral enhancement mechanism should only be present in the Au@Pt-NLTO samples [20,21]. Direct electron transfer depends only on the band alignment at the interface, and can potentially exist in both the Au@Pt-NLTO and Au@Pt-LTO [20,21].
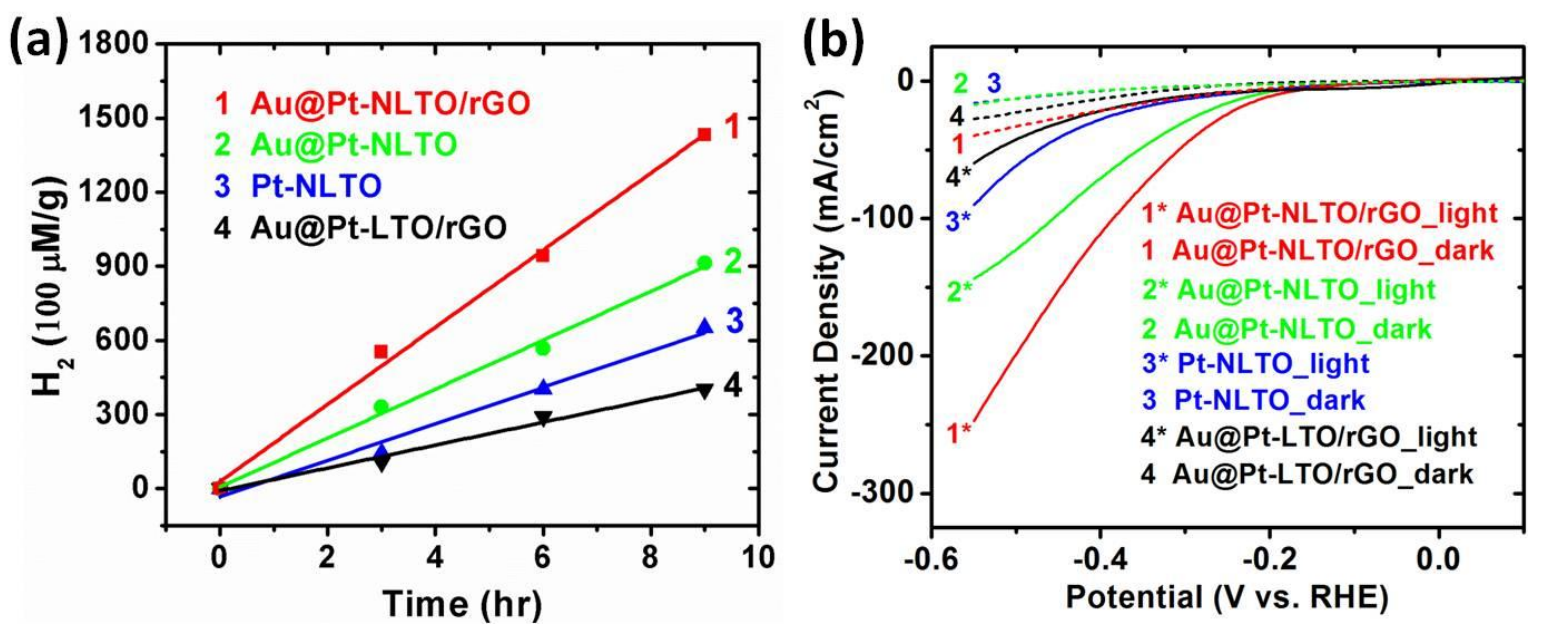

Figure 7-4. Hydrogen generation rate and photocurrent. (a) The interaction of plasmonics and the doped Pt-NLTO with and without heterostructuring with rGO is seen to outperform the undoped Au@PtLTO/rGO. (b) Similarly, the largest photocurrent is seen for the Au@Pt-NLTO/rGO sample, with the Au@Pt-NLTO and Pt-NLTO both outperforming the doped Au@Pt-LTO/rGO samples. The hydrogen generation and photocurrent show the strong enhancement of combined doping and plasmonics.

The photocatalytic production of hydrogen under simulated solar irradiation is shown in Figure 7-4 (a). Ethanol was used as the hole scavenger in all photocatalysis experiments. The undoped sample Au@Pt-LTO/rGO showed the lowest hydrogen production rate, despite the possibility of plasmonic enhancement by DET and a charge extraction enhancement by the rGO. After nitrogen doping, the Pt-NLTO showed a greater hydrogen production rate than the Au@Pt-LTO/rGO due to the increased spectral 
utilization. When the Au nanoparticles are deposited on the Au@Pt-NLTO the hydrogen production rate is increased by $\sim 150 \%$ when compared to the Pt-NLTO alone. Since a large plasmonic enhancement was present only when the NLTO and LSPR overlapped spectrally, but not in the Au@Pt-LTO sample without spectral overlap, the enhancement is most likely due to PIRET. However, it is also possible that the Fermi level equilibration is increasing the carrier lifetime and increasing the reductive potential [2224].

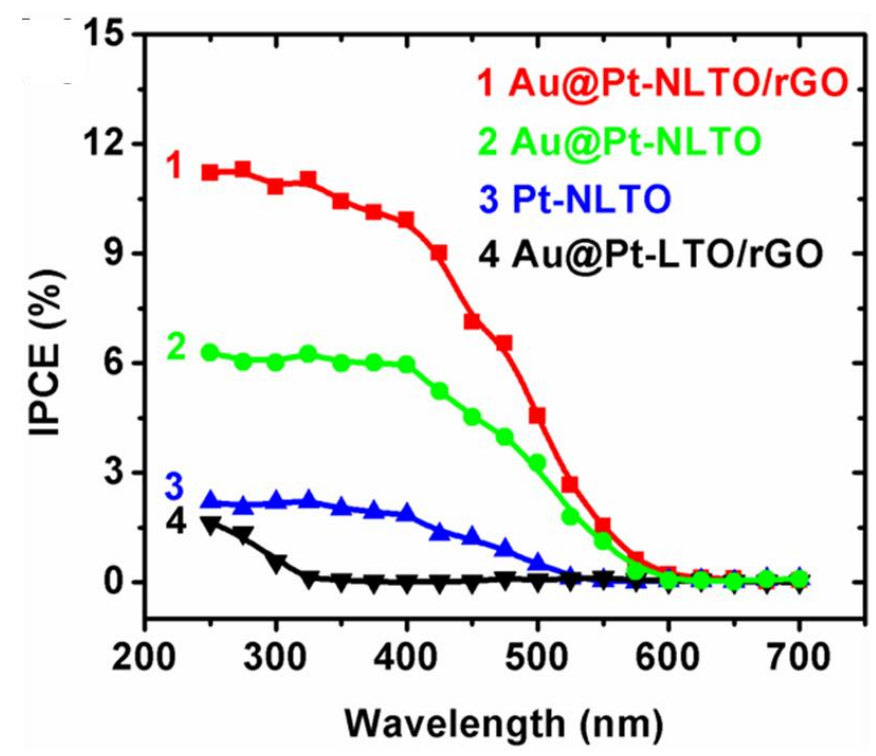

Figure 7-5. Photoelectrochemical performance of the four sample: IPCE curves.

In Figure 7-5, the PEC activity of the photoelectrode against the wavelength of the irradiation light was evaluated according to the incident photon-to-electron conversion efficiency (IPCE), which was measured at a bias of $-0.25 \mathrm{~V}$ vs. RHE from $250 \mathrm{~nm}$ to $700 \mathrm{~nm}$ with an interval of $25 \mathrm{~nm}$. Compared with the nitrogen doped samples, the onset point of the IPCE of the sample Au@Pt-LTO/rGO initiated from $325 \mathrm{~nm}$. However, for the nitrogen doped samples, the onset points of the IPCE curves were 112 
extended to visible light range. The onset points of the IPCEs reflected the difference of band gaps due to nitrogen doping. Moreover, both of the IPCE of the Au @Pt-NLTO/rGO and the Au@Pt-NLTO started to rise from zero at $600 \mathrm{~nm}$, however, the IPCE was dropped to zero at $525 \mathrm{~nm}$ for the Pt-NLTO, which was in agreement of light absorption curves in Figure 1 (d).

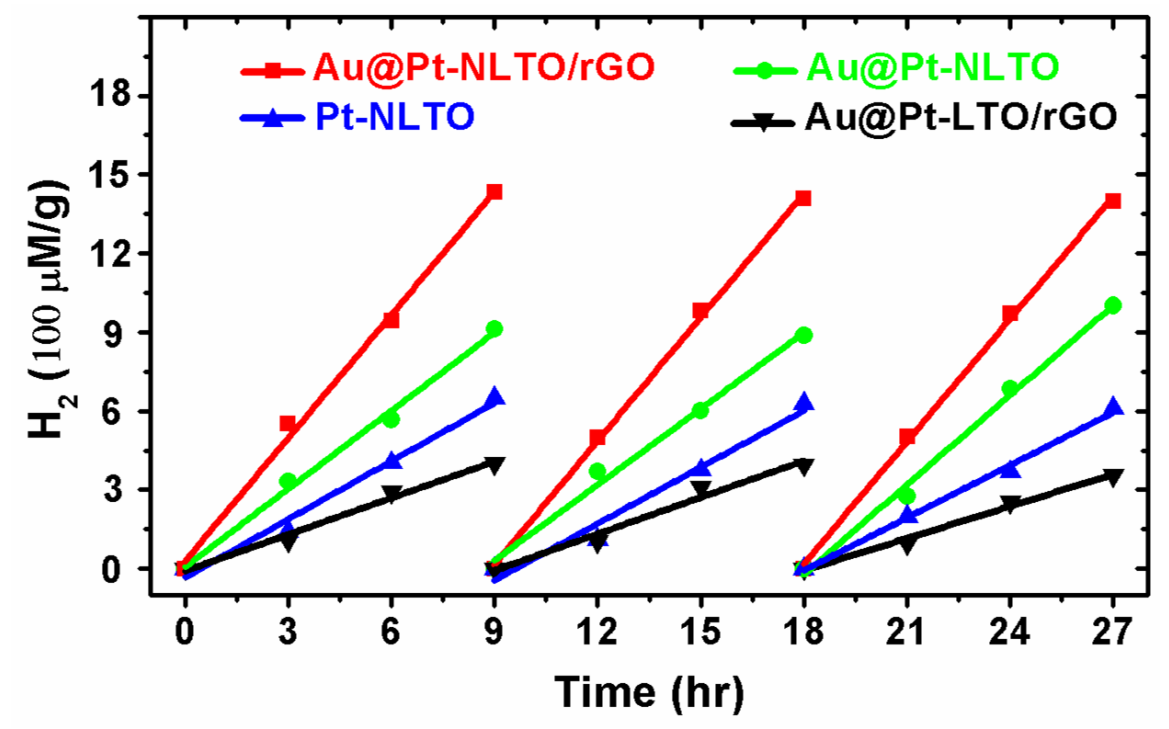

Figure 7-6 Hydrogen generation rate over three cycles for Au@Pt-NLTO/rGO, Au@Pt-NLTO, Pt-NLTO, and Au@Pt-LTO/rGO. 
Table 7-2 The photocatalytic hydrogen generation rates and BET surface areas of the four samples.

\begin{tabular}{|c|c|c|c|}
\hline Sample & $\mathbf{H}_{\mathbf{2}}\left(\boldsymbol{\mu} \mathbf{M} \cdot \mathbf{g}^{-\mathbf{1}} \cdot \mathbf{h}^{-\mathbf{1}}\right)$ & $\begin{array}{c}\text { Surface areas } \\
\left(\mathbf{m}^{2} \cdot \mathbf{g}^{-1}\right)\end{array}$ & $\mathbf{H}_{\mathbf{2}}\left(\boldsymbol{\mu M} \cdot \mathbf{m}^{-\mathbf{2}} \cdot \mathbf{h}^{-\mathbf{1}}\right)$ \\
\hline Au@Pt-NLTO/rGO & 163.4 & 44.65 & 3.66 \\
\hline Au@Pt-NLTO & 101.1 & 38.54 & 2.62 \\
\hline Pt-NLTO & 65.3 & 40.25 & 1.62 \\
\hline Au@Pt-LTO/rGO & 44.2 & 43.53 & 1.02 \\
\hline
\end{tabular}

After addition of rGO to the Au@Pt-NLTO sample the hydrogen production rate again increased by $250 \%$ compared to the Pt-NLTO alone because of the increased charge extraction. [40-42] The hydrogen production rate showed that the doping and plasmonic enhancements act synergistically, enhancing the performance beyond that of the undoped Au@Pt-LTO/rGO sample. All four samples showed stable and recyclable photocatalytic properties in three continuous experiments of photocatalytic hydrogen generation (Figure 7-6). The average rates of photocatalytic hydrogen generation and BET surface areas of the four samples were collected in Table 7-2, showing the difference in photocatalytic activity between samples was not caused by differences in surface area. The photocurrent (Figure 7-4 (b)) is seen to follow the same increasing trend as the hydrogen production rate with doping, addition of the plasmon, and addition of the rGO synergistically increasing the photocurrent beyond that of the Au@PtLTO/rGO before doping. 


\subsection{Conclusions}

In conclusion, it has been shown that doping and plasmonics act synergistically to increase photocatalytic hydrogen generation and photocurrent beyond that of an undoped sample combined treated by the same enhancement techniques. The Fermi level equilibration of the Au NP both with and without the charge extraction of the rGO acts to increase carrier lifetime and increase the reductive potential. The plasmon overlaps with the doped NLTO spectrally, leading to PIRET and an increase in spectral utilization. These effects combine to increase the hydrogen generation rate from 44.2 to $163.4 \mu \mathrm{M} \cdot \mathrm{g}^{-}$ ${ }^{1} \cdot \mathrm{h}^{-1}$. The reported results can be used to guide the optimization of the plasmonic effect with doping in other nanomaterial photocatalysts for enhancement of hydrogen generation.

\section{Reference}

[1] Fujishima, A.; Honda, K. Nature 1972, 238, 37.

[2] Kudo, A.; Miseki, Y., Chem. Soc. Rev., 2009, 38, 253.

[3] Zou, Z. G., Ye, J. H., Sayama, K., Arakawa, H., Nature, 2001, 414, 625-627.

[4] Chen, X. B., Shen, S. H., Guo, L. J., Mao, S. S., Chem. Rev., 2010, 110, 6503-6570.

[5] Li, J. T.; Meng, F. K.; Suri, S.; Ding, W. Q.; Huang, F. Q.; Wu, N. Q. Chem. Commun. 2012, 48, 8213.

[6] Wang, X. C., Maeda, K., Thomas, A., Takanabe, K., Xin, G., Carlsson, J. M., Domen, K., Antonietti, M., Nat. Mater.,2009, 8, 76-80. 
[7] Meng, F. K.; Li, J. T.; Hong, Z. L.; Zhi, M. J.; Sakla, A.; Xiang, C. C.; Wu, N. Q. Catal. Today. 2013, 199, 48.

[8] Chen, X. B., Liu, L., Yu, P. Y., Mao, S. S., Science, 2011, 331, 746-750.

[9] Meng, F. K.; Li, J. T.; Cushing, S. K.; Bright, J.; Zhi, M. J.; Rowley, J. D.; Hong, Z. L.; Manivannan, A.; Bristow, A. D.; Wu, N. Q. ACS Catal. 2013, 3, 746.

[11] Abe, R., Higashi, M., Domen, K., J. Am. Chem. Soc., 2010, 132, 11828-11829.

[12] Meng, F.K., Li, J. T., Cushing, S. K., Zhi, M. J., Wu, N. Q., J. Am. Chem. Soc. 2013, $135,10286-10289$

[13] Kay, A.; Cesar, I.; Gratzel, M., J. Am. Chem. Soc. 2006, 128, 15714.

[14] Xiang, Q. J.; Yu, J. G.; Jaroniec, M. J. Am. Chem. Soc. 2012, 134, 6575.

[15] Li, Q., Guo, B. D., Yu, J. G., Ran, J. R., Zhang, B. H., Yan, H. J., Gong, J. R., J. Am. Chem. Soc. 2011, 133, 10878-10884.

[16] Meng, F. K.; Hong, Z. L.; Arndt, J.; Li, M.; Zhi, M. J.; Yang, F.; Wu, N. Q. Nano Res. 2012, 5, 213.

[17] Wu, N. Q.; Wang, J.; Tafen, D. N.; Wang, H.; Zheng, G. Z.; Lewis, J. P.; Liu, X. G.; Leonard, S. S.; Manivannan, A. J. Am. Chem. Soc. 2010, 132, 6679.

[18] M. Li, J. Zhang, W. Dang, S. K. Cushing, D. Guo, N. Q. Wu, P. Yin, Phys. Chem. Chem. Phys. 2013, 15, 16220-16226.

[19] Li, J. T., Cushing, S. K., Zheng, P., Meng, F. K., Chu, D., Wu, N. Q., Nature Comm. 2013, 4, 2651.

[20] Cushing, S. K., Li, J. T., Meng, F. K., Senty, T. R., Suri, S., Zhi, M. J., Li, M., Bristow, A. D., Wu, N. Q., J. Am. Chem. Soc. 2012, 134, 15033-15041. 
[21] Li, J. T., Cushing, S. K., Bright, J., Meng, F. K., Senty, T. R., Zheng, P., Bristow, A. D., Wu, N. Q., ACS Catal. 2013, 3, 47-51.

[22] Subramanian, V., Wolf, E. E., Kamat, P. V. J. Am. Chem. Soc. 2004, 126, 49434950

[23] Jakob, M., Levanon, H., Kamat, P. V. Nano Lett., 2003, 3, 353-358.

[24] Rosseler, O., Shankar, M. V., Karkmaz-Le Du, M., Schmidlin, L., Keller, N., Keller, V. J. Catalysis 2010, 269, 179-190

[25] Tada, H., Mitsui, T., Kiyonaga, T., Akita, T., Tanaka, K. Nat. Mat. 2006, 5, 782-786.

[27] Jiang, B. J.; Tian, C. G.; Fan, Q. J.; Jiang, Z.; Wang, J. Q.; Yan, W. S.; Fu, H. G. J. Phys. Chem. C. 2011, 115, 23718.

[28] Zhang, X. Y.; Li, H. P.; Cui, X. L.; Lin, Y. H. J. Mater. Chem. 2010, 20, 2801.

[29] Xiang, Q. J.; Yu, J. G.; Jaroniec, M. Chem. Soc. Rev. 2012, 41, 782.

[30] Williams, G.; Seger, B.; Kamat, P. V. ACS Nano 2008, 2, 1487.

[31] Ng, Y. H.; Iwase, A.; Kudo, A.; Amal, R. J. Phys. Chem. Lett. 2010, 1, 2607.

[32] Li, K. W.; Wang, Y.; Wang, H.; Zhu, M. K.; Yan, H., Nanotechnology, 2006, 17, 4863.

[33] Kasahara, A.; Nukumizu, K.; Takata, T.; Kondo, J. N.; Hara, M.; Kobayashi, H.; Domen, K., J. Phys. Chem. B., 2003, 107, 791-797.

[34] Ahn, P. A.; Shin, E. C.; Jo, J. M.; Yu, J. H.; Woo, S. K.; Lee, J. S., Fuel Cells, 2012, 12, 1070-1084.

[35]Canales-Vazquez, J.; Tao, S. W.; Irvine, J. T. S., Solid State Ionics, 2003, 159, 159165. 
[36] J.F. Moulder, W.F. Stickle, P.E. Sobol, K.D. Bomben, Handbook of X-ray Photoelectron Spectroscopy, Eden Prairie, 1992.

[37] Li, N.; Liu, G.; Zhen, C.; Li, F.; Zhang, L. L.; Cheng, H. M. Adv. Funct. Mater. 2011, 21, 1717.

[38] Link, S., EI-Sayed, M., J. Phys. Chem. B., 1999, 103, 8410-8426.

[39] Link, S., EI-Sayed, M., J. Phys. Chem. B., 1999, 103, 4212-4217.

[40] Lightcap, I. V.; Kosel, T. H.; Kamat, P. V. Nano Lett. 2010, 10, 577.

[41] Geim, A. K., Novoselov, K. S., Nat. Mater. 2007, 6, 183-191.

[42] Geim, A. K., Science, 2009, 324, 1530-1534. 


\section{Chapter 8 Solar Hydrogen Generation by Nanoscale p-n}

\section{Junction of p-type Molybdenum Disulfide/n-type Nitrogen-}

\section{Doped Reduced Graphene Oxide}

\subsection{Background and introduction}

It is of great interest to utilize solar energy to produce hydrogen from renewable resources such as water [1-5]. In Chapters 3-7 we have shown how doping, charge extraction, and plasmonics can all lead to enhancements in hydrogen generation for both large and small band gap metal oxide photocatalysts. However all of these materials have relied on precious metals such as $\mathrm{Pt}, \mathrm{Rh}$ and $\mathrm{Pd}$ are essential components in the catalysts for hydrogen evolution reaction (HER). The high cost and scarcity of these precious metals counters the benefits gained from an otherwise environmentally sustainable energy source $[1,6,7]$. It is vital to find inexpensive, earth-abundant materials to replace precious metal catalysts for sustainable development. Molybdenum disulfide $\left(\mathrm{MoS}_{2}\right)$ is a nontoxic, environmentally friendly, abundant semiconductor with the potential to fill this void. $\mathrm{MoS}_{2}$ is widely used as an electrocatalyst for $\operatorname{HER}[1,8,9] . \mathrm{MoS}_{2}$ alone has negligible photocatalytic activity despite the fact that bulk $\mathrm{MoS}_{2}$ has an indirect absorbance edge at $\sim 1040 \mathrm{~nm}$ [10], which seems ideal for solar light harvesting. It remains unclear why $\mathrm{MoS}_{2}$ is effective as an electrocatalyst but inactive as a photocatalyst. However, when $\mathrm{MoS}_{2}$ is combined with other co-catalysts it does exhibit photocatalytic activity toward HER [11-16]. For example, $\mathrm{MoS}_{2}$ has shown 
photocatalytic activity when it was coupled with $\mathrm{Ru}(\mathrm{bpy})^{32+}$-based photosensitizer molecules [14], indicating that insufficient charge separation in $\mathrm{MoS}_{2}$ may limits its photoactivity. Similarly, $\mathrm{MoS}_{2}$ has been used as a co-catalyst with CdS [11]. Unfortunately $\mathrm{CdS}$ is toxic and instable under photochemical reaction.

Graphene and reduced graphene oxide (rGO) have been incorporated with semiconductors to form heterostructures to improve the catalytic activity in either photocatalysis [17-26] or electrocatalysis8. For example, $\mathrm{MoS}_{2}$ nanoparticles (NPs) supported on rGO have been proven to be active HER electrocatalysts8. Recently, Eosin $\mathrm{Y}$ dye and the $\mathrm{MoS}_{2}$ NPs have been co-deposited on the rGO nanosheets to form a photocatalyst [26]. However, the $\mathrm{MoS}_{2}$-rGO system shows no photocatalytic activity in the absence of a photosensitizer (Eosin $\mathrm{Y}$ dye), which indicates that $\mathrm{MoS}_{2}$ provides reaction sites for HER but it does not harvest solar energy. In the semiconductor-rGO composite photocatalysts reported previously, rGO was treated as a metallic support to allow conduction. It was always considered as a passive component that provided a channel for charge transport, not help with the charge generation.

$\mathrm{MoS}_{2}$ has a layered S-Mo-S structure, which has extremely low conductivity between adjacent S-Mo-S layers1, leading to poor charge mobility. Bulk $\mathrm{MoS}_{2}$ has poor catalytic activity toward HER. For nano-sized $\mathrm{MoS}_{2}$, the edges of the S-Mo-S layers are the active sites for HER activity [27,28]. Two strategies have been proposed to improve the HER catalytic activity of $\mathrm{MoS}_{2}$ [29,30]: (i) increase the number of active sites, and (ii) promote the charge transport. In this paper, a thin nanoplatelet structure of $\mathrm{MoS}_{2}$ will be fabricated to create a large percentage of edge sites. This nanoplatelet structure will also shorten the distance that photo-generated charge carriers diffuse to the reaction sites, 
lowering the charge recombination loss that usually occurs during volume diffusion. The nanoplatelet geometry will also increase the contact area with the rGO sheets as compared to the spherical particles that are only in point contact with rGO. The increased contact area will promote the charge transfer at the $\mathrm{MoS}_{2} / \mathrm{rGO}$ interface. In addition, the rGO nanosheets will be doped with nitrogen to form a n-type semiconductor as a support to grow the $\mathrm{p}$-type $\mathrm{MoS}_{2}$ nanoplatelets, forming many nanoscale $\mathrm{p}$-n junctions on each rGO nanosheet. The space charge layer created by the nanoscale p-n junctions will not only suppress the charge recombination but also enhance the photo-generation of electron-hole pairs. In this photocatalyst system, $\mathrm{MoS}_{2}$ not only acts as a catalytic center but also as a photocenter for absorbing solar light to generate charge carriers. As a result, the nanoscale $p-n$ junction increases the photocatalytic activity toward HER. Furthermore, no noble metals or toxic materials are used in the $p-\mathrm{MoS}_{2} / n-\mathrm{rGO}$ photocatalyst. This work presents an effective method to improve the HER photocatalysis of semiconductors without sacrificing environmental sustainability by incorporating precious metals or toxic co-catalysts.

\subsection{Experimental section}

\subsubsection{Material synthesis}

Graphene oxide (GO) was synthesized by the established Hummer method [25].

The $\mathrm{MoS}_{2} /$ reduced graphene oxide (rGO) was synthesized by the hydrothermal method [8,9]. Briefly, $132 \mathrm{mg}$ of $\left(\mathrm{NH}_{4}\right)_{2} \mathrm{MoS}_{4}(>99 \%$, Sigma-Aldrich), $60 \mathrm{mg}$ of GO and $0.6 \mathrm{~g}$ of L-Ascorbic acid (L-AA, >99\%, Sigma-Aldrich) was dissolved into $60 \mathrm{~mL}$ of N, N- 
Dimethylformamide (DMF, anhydrous, 99.8\%, Alfa-Aesar) and then sonicated for 20 minutes to form a homogenous solution. The solution was then transferred to a $75 \mathrm{~mL}$ of Teflon-lined autoclave, and heated at $200{ }^{\circ} \mathrm{C}$ for $10 \mathrm{~h}$. After hydrothermal treatment, the autoclave was cooled down to room temperature and the product was collected. Subsequently, the product was washed with DI water and then re-collected by centrifugation at $13000 \mathrm{rpm}$ for $10 \mathrm{~min}$. The washing-centrifuging step was repeated seven times to remove DMF. Finally the wet product was dried at $50{ }^{\circ} \mathrm{C}$ overnight to obtain the $\mathrm{MoS}_{2} / \mathrm{rGO}$ powder sample. The solitary $\mathrm{MoS}_{2}$ sample was obtained with a similar hydrothermal method except that the GO and L-AA was added into the solution.

Nitrogen-doping was performed on the as-prepared $\mathrm{MoS}_{2} / \mathrm{rGO}$ sample. The $\mathrm{MoS}_{2} / \mathrm{rGO}$ was heated in a $\mathrm{NH}_{3}$ flow $(100 \mathrm{~mL} / \mathrm{min})$ to $500{ }^{\circ} \mathrm{C}$ at a ramp rate of $5{ }^{\circ} \mathrm{C} / \mathrm{min}$, and then held at $500{ }^{\circ} \mathrm{C}$ in $\mathrm{NH}_{3}$ for $30 \mathrm{~min}$ in order to dope nitrogen into rGO. It should be pointed out that GO was simultaneously reduced to rGO during the nitrogen-doping process. Finally, the $p-\mathrm{MoS}_{2} / n-\mathrm{rGO}$ sample with the nanoscale $p$ - $n$ junctions was obtained.

\subsubsection{Material characterization}

The shape and dimension of samples were characterized with a field-emission scanning electron microscope (SEM, JEOL 7600F) and a transmission electron microscope (TEM, JEOL JEM 2100F). For preparation of SEM samples, $1 \mathrm{mg}$ of powder sample was mixed with $1 \mathrm{~mL}$ of de-ionized water to form a suspension, which was then ultrasonated for 30 seconds. The suspension was then deposited onto a pre-cleaned silicon wafer substrate (SPI Inc.), and dried in air. For preparation of TEM samples, the 
powder was mixed with ethanol and deposited onto a TEM grid (Ted Pella, Inc.), and then dried at room temperature overnight. The crystal structures of the samples were characterized by a high-resolution TEM (HRTEM) and X-ray diffraction (XRD, X' Pert Pro PW3040-Pro, Panalytical Inc.) with $\mathrm{Cu} \mathrm{K \alpha}$ radiation. The element composition of samples and the chemical status of N, C, S and Mo were analyzed with X-ray photoelectron spectroscopy (XPS, PHI 5000 Versa Probe system, Physical Electronics) was used. The binding energies in all XPS spectra were calibrated with a reference to the C 1s peak at $284.8 \mathrm{eV}$. The light absorption properties of samples were evaluated by an ultraviolet-visible spectrometer (UV-Vis, Lambda 750, PerkinElmer Inc.). The surface areas of the samples were measured by the Brunauer, Emmett and Teller (BET) method with the instrument of Micromeritics ASAP 2020.

\subsubsection{Photocatalytic $\mathrm{H}_{2}$ generation}

A commercial solar simulator (300 W, Newport) equipped with a Xenon lamp and an AM 1.5G filter was employed as the light source for photocatalytic $\mathrm{H}_{2}$ generation. At First, $10 \mathrm{mg}$ of photocatalyst sample was mixed with $10 \mathrm{~mL}$ of de-ionized water and $10 \mathrm{~mL}$ of ethanol to form a homogenous solution. The solution was then transferred to a $100 \mathrm{~mL}$ of quartz flask, and bubbled with pure $\mathrm{N}_{2}$ for 2 min. Subsequently, the quartz flask was sealed with a septum stopper. The quartz flask was placed in front of the simulated solar light with the intensity of $40-50 \mathrm{~mW} / \mathrm{cm}^{2}$ with the irradiation area of about $1.8 \mathrm{~cm}^{2}$. The flask was exposed to the light for $4,8,12,16$ and $20 \mathrm{~h}$, respectively. Finally, the gas composition was measured with a gas chromatography (GC) equipped with a thermal conductivity detector (TCD) from $5 \mathrm{~mL}$ of gas sample extracted from the 
flask.

Photocatalytic $\mathrm{H}_{2}$ generation under natural sun light irradiation was performed on the Evansdale campus of West Virginia University, Morgantown, WV, USA. $\left(39^{\circ} 38^{\prime} 1^{\prime \prime} \mathrm{N}\right.$ $\left.79^{\circ} 57^{\prime} 2^{\prime \prime} \mathrm{W}\right)$. The experiments were run from 12:00 pm to 2:00 pm on September 21th, 2012. Sunlight incident angle was about $45.2^{\circ}$ at noon. The intensity of the sun light was recorded. Three batches of $p-\mathrm{MoS}_{2} / n$-rGO samples were used for photocatalytic $\mathrm{H}_{2}$ generation simultaneously.

\subsubsection{Photoelectrode fabrication and photoelectrochemical measurement}

Photoelectrode fabrication: $0.1 \mathrm{~g}$ of photocatalyst sample was mixed with $0.5 \mathrm{~mL}$ of terpineol ( $\sim 95 \%$, Sigma-Aldrich) and stirred with a magnetic stirrer for $24 \mathrm{~h}$. The suspension was dip-coated onto the fluorine-doped tin oxide (FTO) glass substrate (Hartford, TEC 15). A doctor blading technique was employed to ensure the same thickness for each photoelectrode. The photoelectrode was then dried on a hot plate at $80{ }^{\circ} \mathrm{C}$, and then heated in a quartz tubular furnace in nitrogen at $550{ }^{\circ} \mathrm{C}$ for $2 \mathrm{~h}$. A silver wire was then connected to the FTO substrate with the silver colloid paste. Finally epoxy was solidified to cover the FTO substrate, the silver paste and wire to avoid short current in the measurement.

Photoelectrochemical measurement: A Photoelectrochemical cell (PEC) with a three-electrode configuration was constructed. In the PEC, the Pt wire and $\mathrm{Ag} \mid \mathrm{AgCl}$ were used as the counter electrode and the reference electrode, respectively. Photoelectrochemical performance of the photoelectrode was measure in $1 \mathrm{M} \mathrm{NaOH}$ (>98.0\%, Sigma-Aldrich) aqueous electrolyte $(\mathrm{pH}=13.6)$. The electrolyte was bubbled 
with $\mathrm{N}_{2}$ for 30 min prior to PEC measurement. The light source used in the PEC measurements was identical with the simulated light used in the photocatalytic $\mathrm{H}_{2}$ generation testing.

The $J-V$ curves were obtained with a Gamry electrochemical station (Reference $\left.3000^{\mathrm{TM}}\right)$. The obtained potentials versus $\mathrm{Ag} \mid \mathrm{AgCl}$ was converted to the reversible hydrogen electrode (RHE) according to the Nernst Equation 8-1

$$
E_{\text {RHE }}=E+0.05916 p H+E_{0}
$$

where $E_{R H E}$ is the potential vs. RHE, $E_{0}=0.1976 \mathrm{~V}$ at $25{ }^{\circ} \mathrm{C}$, and $E$ is the measured potential vs. $\mathrm{Ag} \mid \mathrm{AgCl}$. The on-off $J-V$ curves were obtained under a bias of $-0.1 \mathrm{~V} v s$. RHE.

To acquire the Mott-Schottky (M-S) plots, impedance spectroscopy was performed at a $\mathrm{AC}$ frequency of $10 \mathrm{kHz}$ in the dark and under simulated light irradiation, respectively. The capacitances was derived from the Equation 8-2

$$
Z_{\text {img }}=1 / 2 \pi f C
$$

where $Z_{\text {img }}$ is the imaginary component of the impedance, $f$ is the frequency and $C$ is the capacitance [2]. The densities of charge carriers was obtained from the slope of M-S plot, according to the Equation 8-3 [2]:

$$
N_{d}=\left(\frac{2}{e_{0} \varepsilon_{0}}\right)\left[\frac{d\left(1 / C^{2}\right)}{d(V)}\right]^{-1}
$$

where $N_{d}$ is the charge carrier density, $C$ is the capacitance of the space charge region, $\varepsilon$ 
is the dielectric constant of the semiconductor, and $\varepsilon_{0}$ is the permittivity of free space.

The incident photo-to-electron conversion efficiency (IPCE) curve was obtained at a bias of $-0.1 \mathrm{~V}$ vs. RHE for all the samples according to the Equation 8-4 [2]:

$$
I P C E=1240 J / \lambda I_{\text {light }}
$$

where the $J$ and $I_{l i g h t}$ are the measured photocurrent and light intensity at the wavelength $\lambda . \lambda$ was varied from $400 \mathrm{~nm}$ to $900 \mathrm{~nm}$ with an interval of $25 \mathrm{~nm}$. 


\subsection{Results and discussion}

\subsubsection{Morphology and crystal structure}
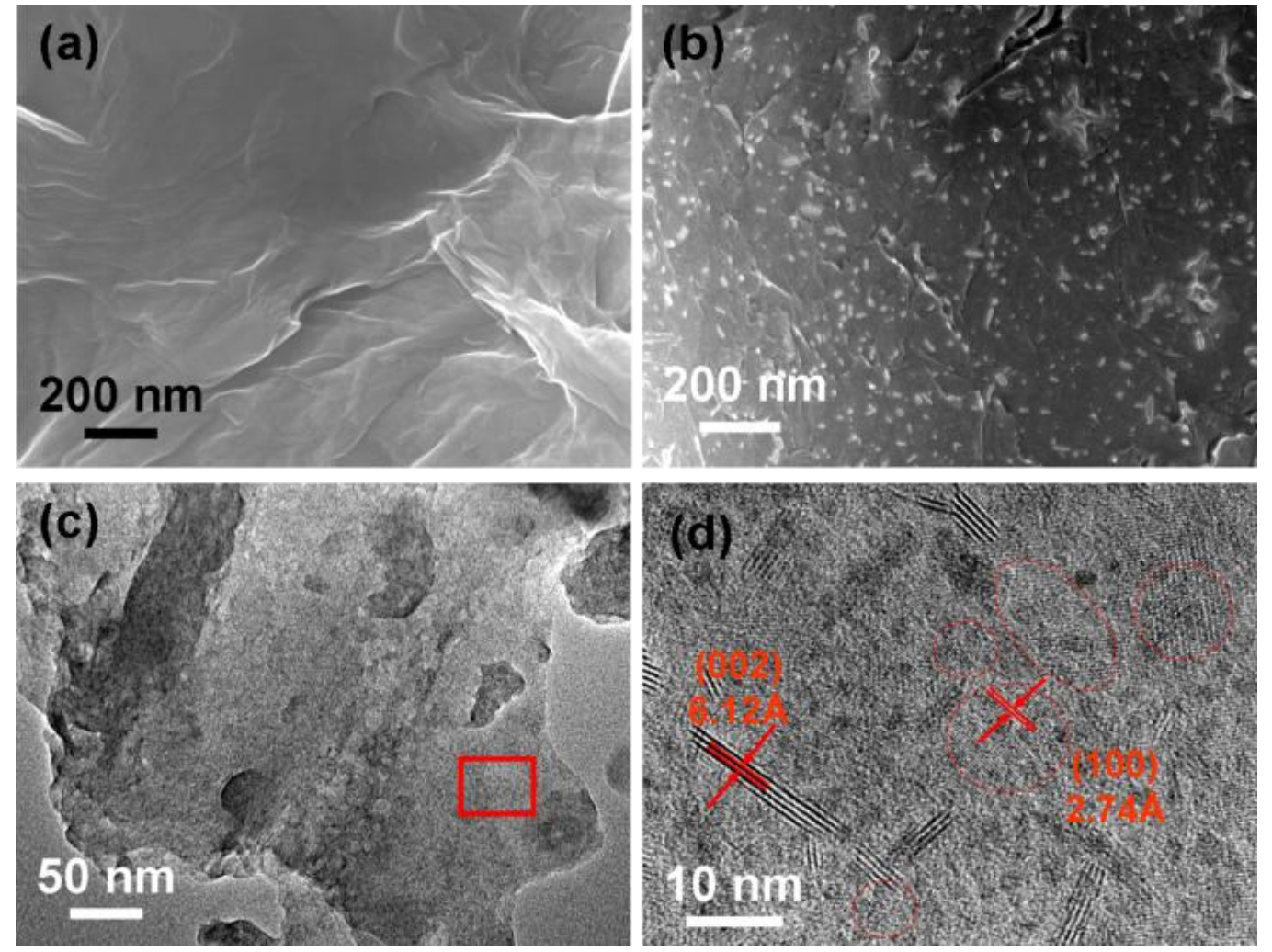

Figure 8-1. SEM images obtained from (a) the rGO sheets, (b) the p-MoS $2 / n-r G O ;$ (c) TEM image of the $\mathrm{p}-\mathrm{MoS}_{2} / \mathrm{n}-\mathrm{rGO}$, and (d) HRTEM image of the $\mathrm{p}-\mathrm{MoS}_{2} / \mathrm{n}$-rGO from the area marked in red square in (c).

Three types of photocatalysts were synthesized in this study, that is, (i) the freestanding solitary $\mathrm{MoS}_{2}$ nanoparticles, (ii) the $\mathrm{MoS}_{2} / \mathrm{rGO}$ composite where the $\mathrm{MoS}_{2}$ nanoplatelets were supported on the undoped $\mathrm{rGO}$ sheets and (iii) the $p-\mathrm{MoS}_{2} / n-\mathrm{rGO}$ junction composite where the $p$-type $\mathrm{MoS}_{2}$ nanoplatelets were deposited on the $n$-type nitrogen-doped rGO semiconductor sheets. All three types of photocatalysts were synthesized with a hydrothermal method. The bare rGO had a smooth flat surface before 
deposition (Figure 8-1 (a)). Figure 8-1 (b) shows that the $\mathrm{MoS}_{2}$ nanoparticles were successfully grown on the nitrogen-doped rGO sheets. The $\mathrm{MoS}_{2}$ nanoparticles were uniformly distributed on the $n$-rGO with a size of 5 20 nm.

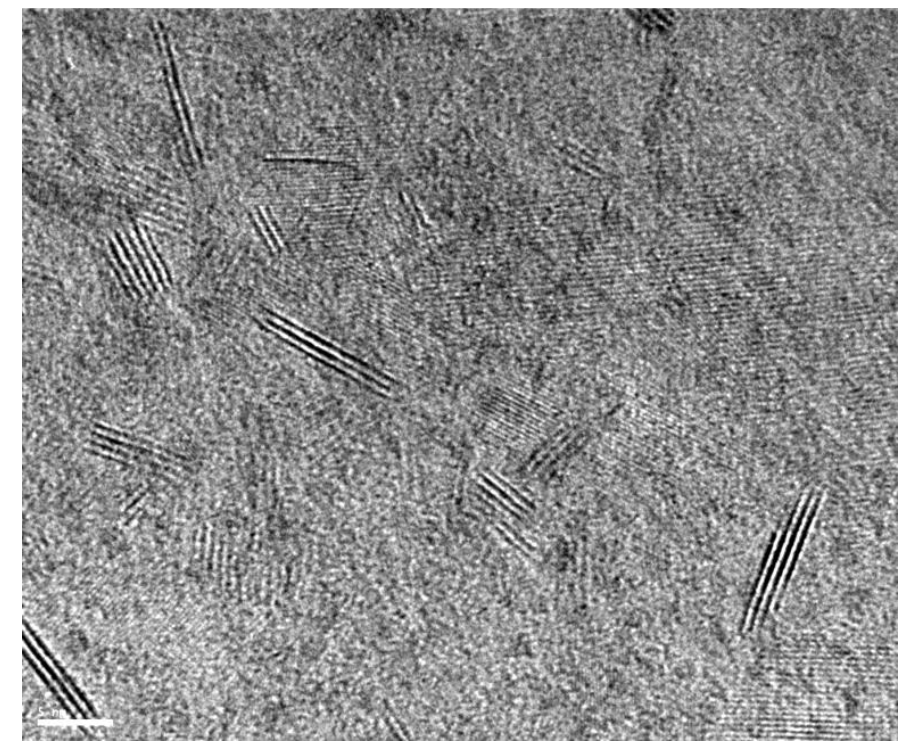

Figure 8-2 TEM image of the $\mathrm{MoS}_{2} / \mathrm{rGO}$ composite in which $\mathrm{rGO}$ was undoped.

Before doping with nitrogen, the $\mathrm{MoS}_{2} / \mathrm{rGO}$ composite in Figure 8-2 showed similar morphology as the $p-\mathrm{MoS}_{2} / n$-rGO junction composite in Figure 8-1 (c) and (d), which indicated the $\mathrm{MoS}_{2}$ was not damaged in the nitrogen-doping process. 

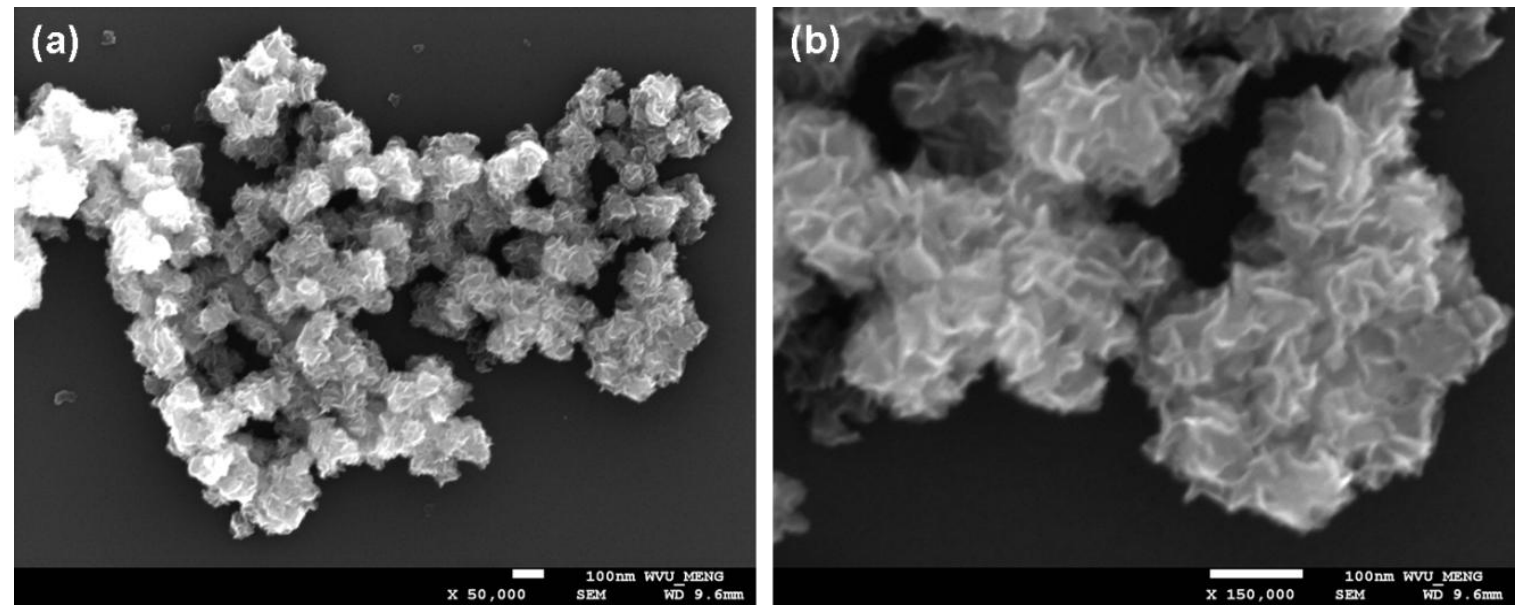

Figure 8-3 SEM images of solitary $\mathrm{MoS}_{2}$.

In contrast, the bare $\mathrm{MoS}_{2}$ without $\mathrm{rGO}$ had a "nanoball" structure composed of $\mathrm{MoS}_{2}$ nanoplatelets with a lateral size of $\sim 80 \mathrm{~nm}$ (Figure 8-3). The results show that the rGO effectively restrained the aggregation of the $\mathrm{MoS}_{2}$ nanoplatelets.

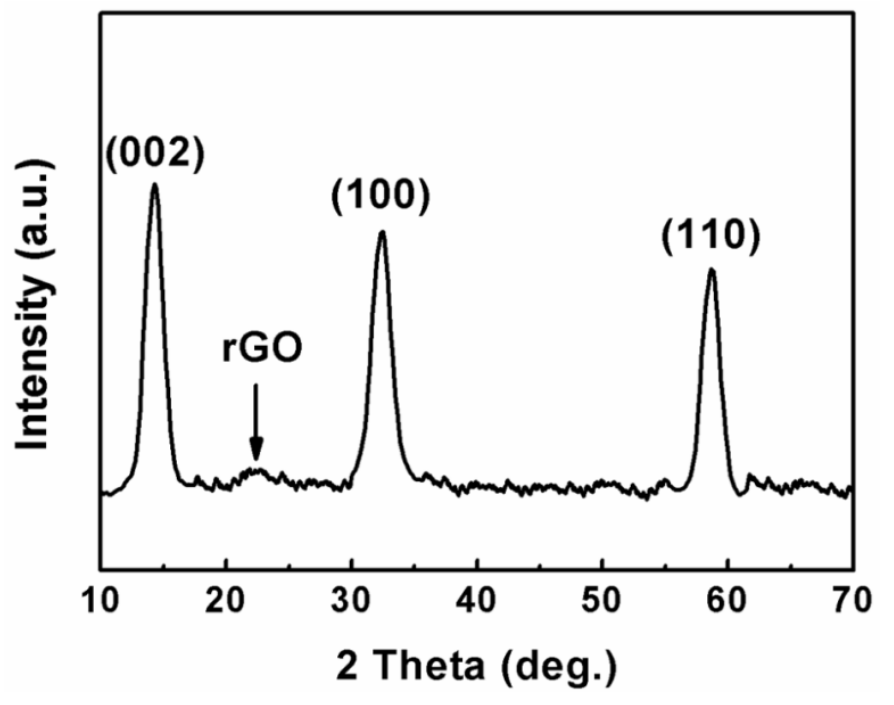

Figure 8-4. XRD pattern of the $p-\mathrm{MoS}_{2} / n-\mathrm{rGO}$ junctioned photocatalyst

The $p$-type $\mathrm{MoS}_{2}$ had a hexagonal structure, as confirmed by the XRD pattern in

Figure 8-4. The broad peaks for the (002), (100) and (110) crystal planes at $14.4^{\circ}, 32.7^{\circ}$ 129 
and $58.4^{\circ}$ indicated that the nanostructured $\mathrm{MoS}_{2}$ was crystalline. A broad peak at $24^{\circ}$ resulted from the rGO [26]. Figure 8-1 (c) shows a TEM image of the p-MoS $2 / n-r G O$. The marked area was enlarged for a HRTEM image in Figure 8-1 (d). The singlecrystalline $\mathrm{MoS}_{2}$ nanoparticles synthesized on the $n$-rGO sheet had a lattice spacing of $6.12 \AA$ and $2.74 \AA$, corresponding to the (002) and (100) facets, respectively (JCPDS: 77 1716).

\subsubsection{Elements compostion}

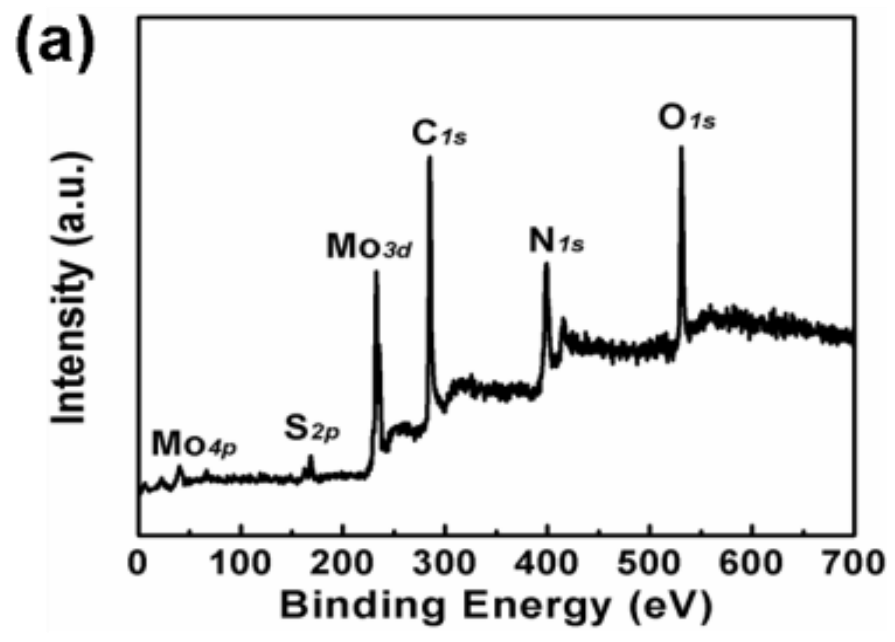

Figure 8-5. XPS spectrum of the $p-\mathrm{MoS}_{2} / n-\mathrm{rGO}$. 



Figure 8-6 XPS spectra of N1s (a), C1s (b), S2p (c) and Mo3d (d). The XPS spectra of the C 1s in (b) was deconvoluted into the $\mathrm{sp}^{2}$ carbon $(284.6 \mathrm{eV}), \mathrm{C}-\mathrm{O}-\mathrm{C}(285.6 \mathrm{eV}), \mathrm{C}-\mathrm{OH}$ or $\alpha-\mathrm{C}$ in $-\mathrm{C}-\mathrm{COOH}(286.7 \mathrm{eV})$, $\mathrm{C}=\mathrm{O}(288.0 \mathrm{eV})$ and $\mathrm{COOH}(289.1 \mathrm{eV})$, respectively [17]

The XPS spectra in Figure 8-5 and Figure 8-6 confirmed the formation of rGO [31-33] and the successful nitrogen doping in the rGO sheets. The doping concentration of $\mathrm{N}$ was estimated to be 6.4 at $\%$ in the $\mathrm{n}-\mathrm{rGO}$ sheets. The molar ratio of $\mathrm{rGO}$ to $\mathrm{MoS}_{2}$ was $10.5: 1$ in the $\mathrm{p}-\mathrm{MoS}_{2} / \mathrm{n}-\mathrm{rGO}$. 


\subsubsection{Light absorption}

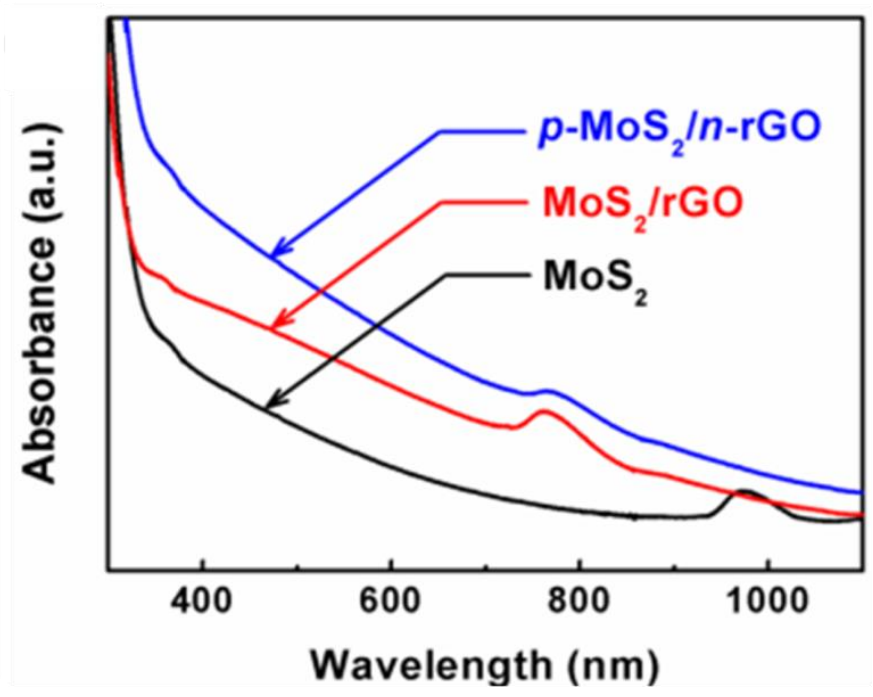

Figure 8-7. UV-Visible absorption spectra of the solitary $\mathrm{MoS}_{2}$, the $\mathrm{MoS}_{2} / \mathrm{rGO}$ and the $\mathrm{p}-\mathrm{MoS}_{2} / \mathrm{n}-\mathrm{rGO}$.

The UV-Visible spectrum in Figure 8-7 shows that solitary $\mathrm{MoS}_{2}$ had an absorbance tail that stretched to $1020 \mathrm{~nm}$, corresponding to the indirect band gap between $\Gamma$ and the middle of the Brillouin zone between $\Gamma$ and $\mathrm{K}[34,35]$. For bulk $\mathrm{MoS}_{2}$, the absorbance edge is typically cut off at $1040 \mathrm{~nm}$ [10]. In addition, solitary $\mathrm{MoS}_{2}$ exhibited weak absorption at around $962 \mathrm{~nm}$. And the $\mathrm{MoS}_{2} / \mathrm{rGO}$ and $p-\mathrm{MoS}_{2} / n-\mathrm{rGO}$ samples had the weak absorption at $781 \mathrm{~nm}$ and $786 \mathrm{~nm}$, respectively. Similar phenomenon was also found in the nano-sized $\mathrm{MoS}_{2}$ particles reported previously $[10,36]$. The weak absorption peaks were due to the direct transition at the $K$ point or to the contributions of excitonic transitions $[34,35]$. 


\subsubsection{Photocatalytic $\mathrm{H}_{2}$ generation}

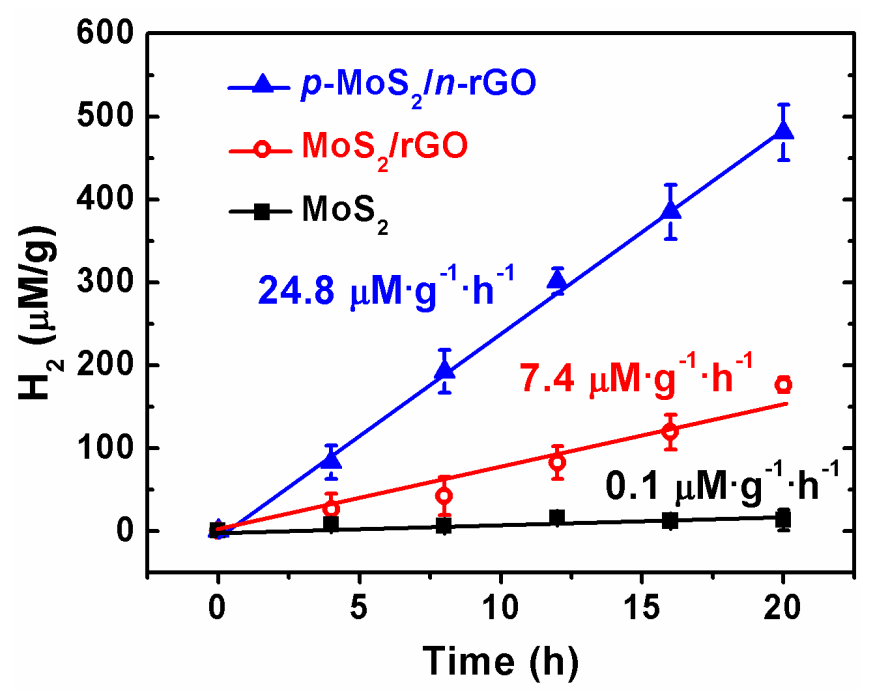

Figure 8-8. Hydrogen generated by the $\mathrm{MoS}_{2}$, the $\mathrm{MoS}_{2} / \mathrm{rGO}$ and the $p-\mathrm{MoS}_{2} / n$-rGO photocatalysts.

For comparative studies, three types of particulate photocatalysts, including the solitary $\mathrm{MoS}_{2}$, the $\mathrm{MoS}_{2} / \mathrm{rGO}$ composite and the $p-\mathrm{MoS}_{2} / n$-rGO junction, were tested in the water/ethanol mixture solution under simulated solar light irradiation (Figure 8-8). Ethanol was a sacrificial reagent that acted as a hole-scavenger. $\mathrm{MoS}_{2}$ alone showed negligible photocatalytic activity toward $\mathrm{H}_{2}$ generation. Although the conduction band alignment of the bulk $\mathrm{MoS}_{2}$ particles is not suitable for water reduction [10,35], the quantum confinement of the nano-sized $\mathrm{MoS}_{2}$ makes it thermodynamically possible to reduce water to hydrogen. The solitary $\mathrm{MoS}_{2}$ has the HER-active edges and absorption properties necessary for HER. However, the lack of activity indicates that either charge separation is extremely inefficient or the photo-generated charges cannot migrate to the HER-active edges. In contrast, the composite $\mathrm{MoS}_{2} / \mathrm{rGO}$, in which the $\mathrm{MoS}_{2}$ nanoparticles were supported on the undoped rGO, showed evident photocatalytic 
activity with a hydrogen generation rate of $7.4 \mu \mathrm{M} \cdot \mathrm{g}^{-1} \cdot \mathrm{h}^{-1}$. Doping nitrogen into the rGO nanosheets led to the formation of hundreds of $p-n$ junctions on each $n$-rGO sheets. As a result, the $\mathrm{H}_{2}$ generation rate further increased to $24.8 \mu \mathrm{M} \cdot \mathrm{g}^{-1} \cdot \mathrm{h}^{-1}$. The surface area of the solitary $\mathrm{MoS}_{2}$ was $134.3 \mathrm{~m}^{2} / \mathrm{g}$, which was even larger than that of the $\mathrm{MoS}_{2} / \mathrm{rGO}(112.3$ $\left.\mathrm{m}^{2} / \mathrm{g}\right)$ and the $p-\mathrm{MoS}_{2} / n-\mathrm{rGO}\left(106.6 \mathrm{~m}^{2} / \mathrm{g}\right)$, negating the surface area effects from explaining the difference in photoactivity. The improvement in photoactivity was solely due to the existence of the $\mathrm{MoS}_{2} / \mathrm{rGO}$ heterostructure.

Table 8-1. The intensity of sun light during photocatalysis tests

\begin{tabular}{|c|c|}
\hline Time & Intensity $\left(\mathbf{W} / \mathbf{m}^{2}\right)$ \\
\hline $12: 00 \mathrm{PM}$ & 882.5 \\
\hline $12: 15 \mathrm{PM}$ & 903.7 \\
\hline $12: 30 \mathrm{PM}$ & 884.7 \\
\hline $12: 45 \mathrm{PM}$ & 878.1 \\
\hline $1: 00 \mathrm{PM}$ & 910.7 \\
\hline $1: 15 \mathrm{PM}$ & 897.1 \\
\hline $1: 30 \mathrm{PM}$ & 910.4 \\
\hline $1: 45 \mathrm{PM}$ & 924.7 \\
\hline $2: 00 \mathrm{PM}$ & 917.1 \\
\hline Average & $\mathbf{9 0 1}$ \\
\hline
\end{tabular}

Location: Morgantown, WV, US (39 $\left.38^{\prime} 1^{\prime \prime N} 79^{\circ} 57^{\prime} 2^{\prime \prime} \mathrm{W}\right)$ [S4]; Date: September 21, 2012; Weather: Sunny; Sunlight incident angle: $45.2^{\circ}$. 
Table 8-2. Normalized $\mathrm{H}_{2}$ generation rates of the $p-\mathrm{MoS}_{2} / n-\mathrm{rGO}$ under sunlight irradiation

\begin{tabular}{|c|c|c|c|c|}
\hline Measurement & No. 1 & No. 2 & No. 3 & Average \\
\hline $\begin{array}{c}\mathbf{H}_{2} \text { generation rate } \\
\left(\mu \mathbf{M ~ h}^{-1} \mathbf{g}^{-1}\right)\end{array}$ & 160.7 & 161.8 & 152.9 & 160.6 \\
\hline
\end{tabular}

The photocatalytic $\mathrm{H}_{2}$ generation rate of the $p-\mathrm{MoS}_{2} / n$-rGO relatively lower than the other materials reported previously $[11,26]$ was due to much lower intensity of irradiation used in the present work. In order to measure the practical performance, the $p$ $\mathrm{MoS}_{2} / n$-rGO sample was exposed to the natural sun light on our campus for $\mathrm{H}_{2}$ generation (Table 8-1 and 8-2). The average hydrogen generation rate was measured to be $160.6 \mu \mathrm{M} \cdot \mathrm{g}^{-1} \cdot \mathrm{h}^{-1}$.

\subsubsection{Photoelectrochemical measurements}
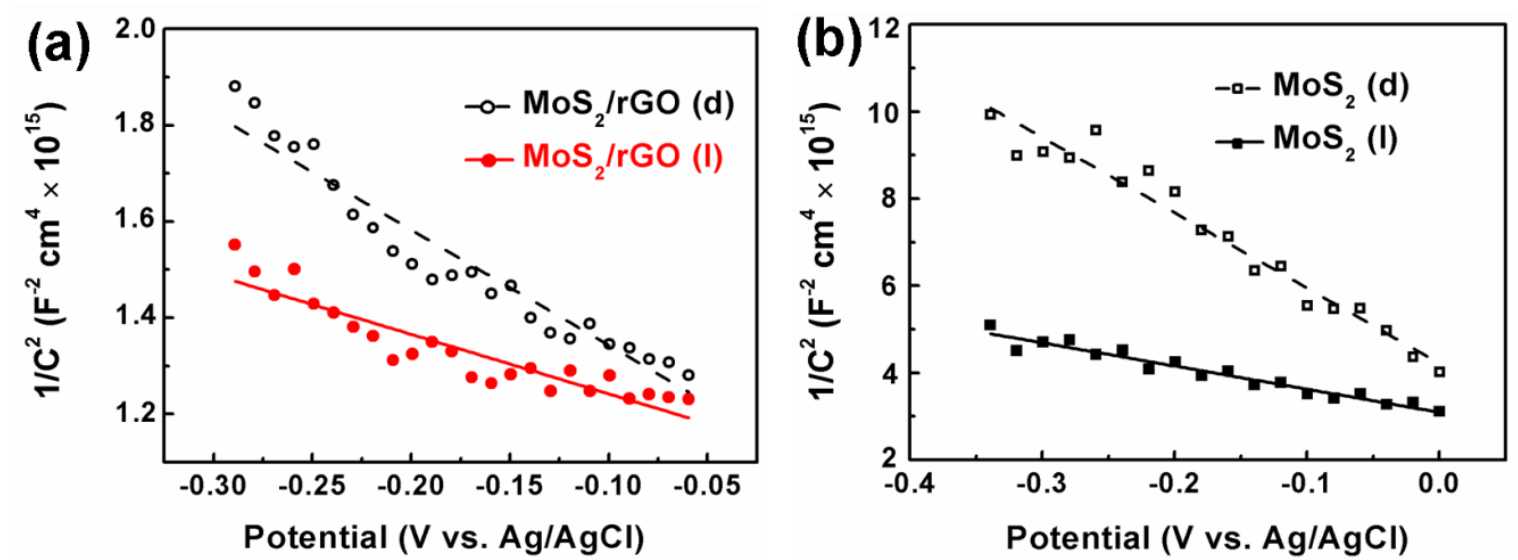

Figure 8-9 Mott-Schottky plots of $\mathrm{MoS}_{2} / \mathrm{rGO}$ (a) and $\mathrm{MoS}_{2}$ (b) in the dark (d) and upon light (l) illumination. 
The three types of particulate photocatalysts were immobilized on fluorine tin oxide (FTO) substrates to form nanoparticle film photoelectrodes. The photoelectrochemical performance of the films was measured in a $1.0 \mathrm{M} \mathrm{NaOH}$ electrolyte solution using a three-electrode photoelectrochemical cell (PEC). To acquire the Mott-Schottky (M-S) plots, impedance spectroscopy was performed at an AC frequency of $10 \mathrm{kHz}$ in the dark and under the simulated solar irradiation, respectively. Both the $\mathrm{MoS}_{2}$ and the $\mathrm{MoS}_{2} / \mathrm{rGO}$ photoelectrodes showed a negative slope for the M-S plots both in the dark and under light irradiation (Figure 8-9), which indicated a $p$-type behavior [37]. The charge carrier densities in the solitary $\mathrm{MoS}_{2}$ were measured to be $3.59 \times 10^{14} \mathrm{~cm}^{-3}$ and $4.67 \times 10^{15} \mathrm{~cm}^{-3}$ in the dark and light irradiation, respectively. While, the charge carrier densities in $\mathrm{MoS}_{2} / \mathrm{rGO}$ were measured to be $9.14 \times 10^{16} \mathrm{~cm}^{-3}$ and $1.82 \times 10^{17} \mathrm{~cm}^{-3}$ in the dark and light irradiation, respectively. It is well known that the $\mathrm{MoS}_{2}$ is a $p$-type semiconductor and the nitrogen-doped rGO is a $n$-type semiconductor $[1,38,39]$. When they were coupled to each other to form a heterostructure, a $p$ - $n$ junction characteristic was observed in the M-S plot where an inverted "V-shape" was present (Figure 8-11). The donor densities in the $p$ - $\mathrm{MoS}_{2} / n$-rGO were $4.22 \times 10^{18} \mathrm{~cm}^{-3}$ in the dark and $7.14 \times 10^{18} \mathrm{~cm}^{-3}$ under light irradiation, respectively. The increased donor density in the $p-\mathrm{MoS}_{2} / n$-rGO indicated that the nanoscale $p$ - $n$ junction created a sufficient space charge layer to enhance the charge carrier creation under light irradiation. 

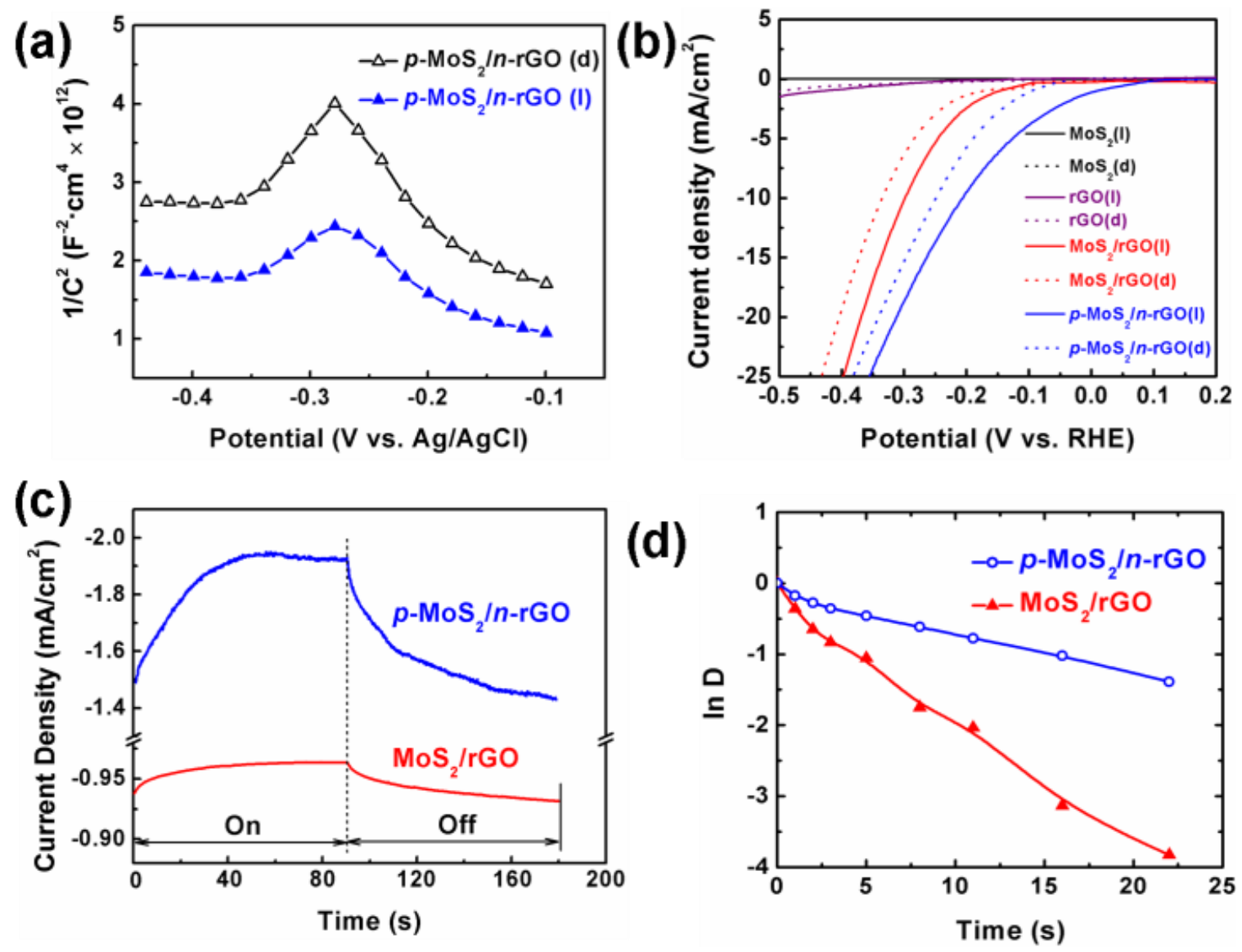

Figure 8-10. (a) Mott-Schottky plots of the p-MoS $2 / n-r G O$ in dark (d) and under light (l) irradiation; (b) JV curves of the various catalysts and the bare rGO in dark (d) and under light (l) irradiation; (c) On-off J-t curves of the $\mathrm{MoS}_{2} / \mathrm{rGO}$ and the $\mathrm{p}-\mathrm{MoS}_{2} / \mathrm{n}$-rGO under light (On) in dark (Off) at $-0.1 \mathrm{~V}$ vs. RHE; (d) normalized plots of the photocurrent-time dependence for the $\mathrm{MoS}_{2} / \mathrm{rGO}$ and the $\mathrm{p}-\mathrm{MoS}_{2} / \mathrm{n}-\mathrm{rGO}$.

The PEC performance was evaluated as shown in the current density $(J)$-potential (V) curve (Figure 8-10). For independent $\mathrm{MoS}_{2}$ or rGO, only a very small photocurrent was observed even at a potential of $-0.5 \mathrm{~V}$ vs. RHE. Under light irradiation, the onset potential for photocurrent generation for the $\mathrm{MoS}_{2} / \mathrm{rGO}$ was $-0.10 \mathrm{~V}$ vs. RHE; and the onset potential for the $p-\mathrm{MoS}_{2} / n$-rGO shifted to $0.05 \mathrm{~V}$ vs. RHE. The onset potential for photocurrent generation typically reflects the catalytic activity of the photoelectrode 
$[40,41]$. The shift of onset potential indicated that the formation of $p-n$ junction improved the catalytic activity toward HER. At a high potential, the photocurrent density reflects the HER rate, which is controlled by the delivery rate of electrons to the electrode/electrolyte interface. Figure 8-10 shows that the photocurrent density was greatly enhanced after $\mathrm{MoS}_{2}$ was coupled to the undoped $\mathrm{rGO}$; and the $p-\mathrm{MoS}_{2} / n-\mathrm{rGO}$ junction further increased the photocurrent density. This indicates that the $p$ - $n$ junction facilitated the charge generation and migration, as well as suppressed the charge recombination $[42,43]$.

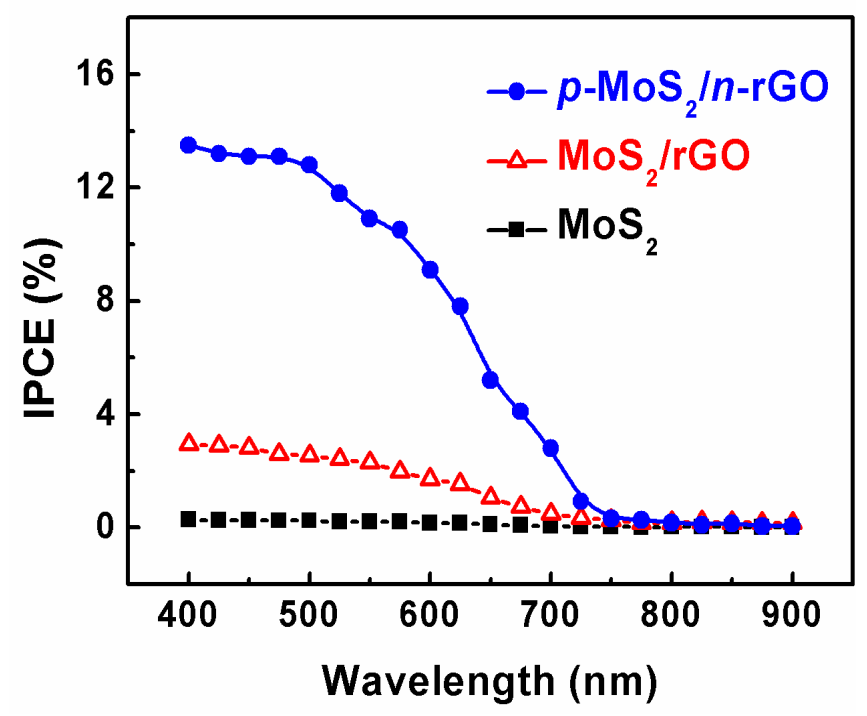

Figure 8-11. IPCE of the $\mathrm{MoS}_{2}$, the $\mathrm{MoS}_{2} / \mathrm{rGO}$ and the $p-\mathrm{MoS}_{2} / n-\mathrm{rGO}$ at a bias of $-0.1 \mathrm{~V}$ vs. RHE.

The photoelectrochemical activity of the photoelectrode as a function of wavelength was quantitatively investigated by the incident photon-to-electron conversion efficiency (IPCE) curve (Figure 8-11), which was measured at a bias of -0.1 V vs. RHE. It is noted that the $\mathrm{p}-\mathrm{MoS}_{2} / \mathrm{n}-\mathrm{rGO}$ junction showed photocatalytic activity toward $\mathrm{H}_{2}$ generation in a wide spectral range from the ultraviolet light through the near-infrared 
light region. Above $780 \mathrm{~nm}$, the photo-response of both the $\mathrm{MoS}_{2} / \mathrm{rGO}$ composite and the $p-\mathrm{MoS}_{2} / n-\mathrm{rGO}$ junction dropped to zero, which was in agreement with the light absorption behavior. At $400 \mathrm{~nm}$, the solitary $\mathrm{MoS}_{2}$ showed negligible photo-response while the IPCE values of the $\mathrm{MoS}_{2} / \mathrm{rGO}$ and the $p-\mathrm{MoS}_{2} / n$-rGO were $2.8 \%$ and $13.6 \%$, respectively. In the $\mathrm{MoS}_{2} / \mathrm{rGO}$ composite, the rGO increases the energy conversion efficiency as a passive charge extraction layer. When a nanoscale $p$ - $n$ junction is formed, the space charge layer creates a built-in electric field and separates the electrons and holes upon light illumination. Hence the $p$ - $n$ junction changes the role of rGO from passive to active, further enhancing the charge separation.

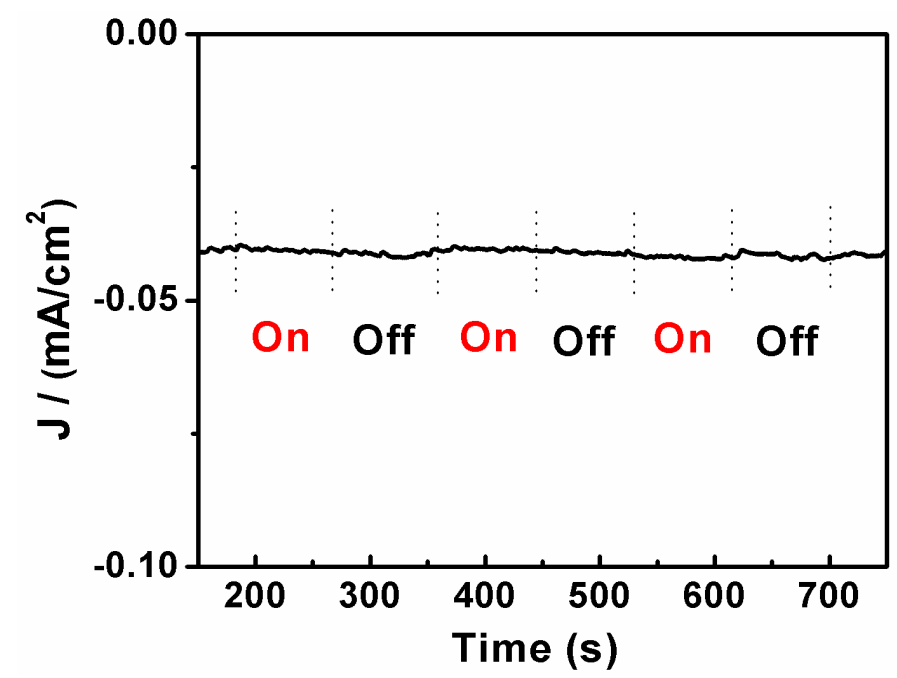

Figure 8-12. On-off $J$ - $t$ curve of $\mathrm{MoS}_{2}$ under a bias of $-0.1 \mathrm{~V}$ vs. RHE.

The hydrogen evolution rate is strongly dependent on the charge recombination rate. The transient photocurrent (chronoamperometric) curve was acquired to investigate the charge recombination behavior (Figure 8-10 (c)). However, no photocurrent was detected for solitary $\mathrm{MoS}_{2}$ in Figure 8-12. The transient photocurrent was measured to 
study the charge recombination behavior. The charge recombination behavior was reflected by a normalized parameter, $D[43,44]$ :

$$
D=\left(I_{t}-I_{s t}\right) /\left(I_{\text {in }}-I_{s t}\right)
$$

where $I_{t}, I_{s t}$, and $I_{\text {in }}$ are the time-dependent, the steady-state and the initial photocurrents respectively. The normalized curve of $\ln D-t$ can be plotted. The transient time constant $(\tau)$ is defined as the time when $\ln D=-1$. Figure 8-10 (d) shows the normalized plots of $\ln D-t$. The transient time constant $(\tau)$ is defined as the time when $\ln D=-1$ [43]. $\tau$ was estimated to be $4.4 \mathrm{~s}$ for the $\mathrm{MoS}_{2} / \mathrm{rGO}$ and $16.2 \mathrm{~s}$ for the $p-\mathrm{MoS}_{2} / n-\mathrm{rGO}$, which confirmed the suppression of charge recombination due to the space charge layer of the nanoscale $p-n$ junction.

\subsection{Conclusions}

In summary, three types of photocatalysts, including solitary $\mathrm{MoS}_{2}$, the $p$ $\mathrm{MoS}_{2} /$ undoped-rGO composite and the $p-\mathrm{MoS}_{2} / n-\mathrm{rGO}$ junction were tested. The solitary $\mathrm{MoS}_{2}$ nanoparticles showed little photocatalytic activity toward HER. The $p-\mathrm{MoS}_{2} / n-\mathrm{rGO}$ junction photocatalyst exhibited much better photocatalytic activity than the $p$ $\mathrm{MoS}_{2}$ /undoped-rGO composite. The rGO was transformed from a passive support to an active component of the heterostructure through doping, which was capable of improving all three vital steps in photocatalysis: charge separation, migration and recombination. The nanoscale $p-n$ junction positively shifted the onset potential, increased both the photocurrent and the hydrogen evolution rate. The $p-\mathrm{MoS}_{2} / n-\mathrm{rGO}$ heterostructure is a 
promising photocatalyst, allowing efficient hydrogen production in a wide spectral range from the ultraviolet light through the near- infrared light region. The $p-\mathrm{MoS}_{2} / n-\mathrm{rGO}$ heterostructure contains only earth-abundant, non-toxic and inexpensive materials. The design principles outlined in this paper can be extended beyond $\mathrm{MoS}_{2}$ and $\mathrm{rGO}$ to

enhance the nanoscale heterostructures undergoing development for solar energy conversion.

\section{Reference}

[1] Laursen, A. B.; Kegnas, S.; Dahl, S.; Chorkendorff, I. Energy Environ. Sci. 2012, 5, 5577.

[2] Li, J. T.; Meng, F. K.; Suri, S.; Ding, W. Q.; Huang, F. Q.; Wu, N. Q. Chem. Commun. 2012, 48, 8213.

[3] Cushing, S. K.; Li, J. T.; Meng, F. K.; Senty, T. R.; Suri, S.; Zhi, M. J.; Li, M.; Bristow, A. D.; Wu, N. Q. J. Am. Chem. Soc. 2012, 134, 15033.

[4] Meng, F. K.; Hong, Z. L.; Arndt, J.; Li, M.; Zhi, M. J.; Yang, F.; Wu, N. Q. Nano Res. 2012, 5, 213.

[5] Li, J. T.; Cushing, S. K.; Bright, J.; Meng, F. K.; Senty, T. R.; Zheng, P.; Bristow, A. D.; Wu, N. Q. ACS Catal. 2013, 3, 47.

[6] Fujishima, A.; Honda, K. Nature 1972, 238, 37.

[7] Meng, F. K.; Li, J. T.; Hong, Z. L.; Zhi, M. J.; Sakla, A.; Xiang, C. C.; Wu, N. Q. Catal. Today. 2013, 199, 48.

[8] Li, Y. G.; Wang, H. L.; Xie, L. M.; Liang, Y. Y.; Hong, G. S.; Dai, H. J. J. Am. Chem. Soc. 2011, 133, 7296. 
[9] Lee, J. K.; Lee, W.; Yoon, T. J.; Park, G. S.; Choy, J. H. J. Mater. Chem. 2002, 12, 614.

[10] Thurston, T. R.; Wilcoxon, J. P. J. Phys. Chem. B. 1999, 103, 11.

[11] Zong, X.; Yan, H. J.; Wu, G. P.; Ma, G. J.; Wen, F. Y.; Wang, L.; Li, C. J. Am. Chem. Soc. 2008, 130, 7176.

[12] Xiang, Q. J., Yu, J. G.; Jaroniec, M. J. Am. Chem. Soc. 2012, 134, 6575.

[13] Zong, X.; Wu. G. P.; Yan, H. J.; Ma, G. J.; Shi, J. Y.; Wen, F. Y.; Wang, L.; Li, C. J. Phys. Chem. C. 2010, 114, 1963.

[14] Zong, X.; Na, Y.; Wen, F. Y.; Ma, G. J.; Yang, J. H.; Wang, D. G.; Ma, Y.; Wang, M.;

Sun, L. C.; Li, C. Chem. Commun. 2009, 153, 4536.

[15] Sobczynski, A. J. Catal. 1991, 131, 156.

[16] Kanda, S.; Akita, T.; Fujishima, M.; Tada, H. J. Colloid Interface Sci. 2011, 354, 607.

[17] Meng, F. K.; Li, J. T.; Cushing, S. K.; Bright, J.; Zhi, M. J.; Rowley, J. D.; Hong, Z. L.; Manivannan, A.; Bristow, A. D.; Wu, N. Q. ACS Catal. 2013, 3, 746.

[18] Jiang, B. J.; Tian, C. G.; Fan, Q. J.; Jiang, Z.; Wang, J. Q.; Yan, W. S.; Fu, H. G. J. Phys. Chem. C. 2011, 115, 23718.

[19] Zhang, X. Y.; Li, H. P.; Cui, X. L.; Lin, Y. H. J. Mater. Chem. 2010, 20, 2801.

[20] Xiang, Q. J.; Yu, J. G.; Jaroniec, M. Chem. Soc. Rev. 2012, 41, 782.

[21] Williams, G.; Seger, B.; Kamat, P. V. ACS Nano 2008, 2, 1487.

[22] Ng, Y. H.; Iwase, A.; Kudo, A.; Amal, R. J. Phys. Chem. Lett. 2010, 1, 2607.

[23] Guo, J. J.; Li, Y.; Zhu, S. M.; Chen, Z. X.; Liu, Q. L.; Zhang, D.; Moon, W. J.; Song, D. M. RSC Adv. 2012, 2, 1356. 
[24] Mukherji, A.; Seger, B.; Qing, G.; Lu, M.; Wang, L. Z. ACS Nano 2011, 5, 3483.

[25] Li, Q.; Guo, B. D.; Yu, J. G.; Ran, J. R.; Zhang, B. H.; Yan, H. J.; Gong, J. R. J. Am. Chem. Soc. 2011, 133, 10878.

[26] Min, S. X.; Lu, G. Q. J. Phys. Chem. C. 2012, 16, 25415.

[27] Hinnemann, B.; Moses, P. G.; Bonde, J.; Jorgensen, K. P.; Nielsen, J. H.; Horch, S.; Chorkendorff, I.; Norskov, J. K. J. Am. Chem. Soc. 2005, 127, 5308.

[28] Jaramillo, T. F.; Jorgensen, K. P.; Bonde, J.; Nielsen, J. H.; Horch, S.; Chorkendorff, L. Science 2007, 317, 100.

[29] Jakob, K.; Chen, Z. B.; Reinecke, B. N.; Jaramillo, T. F. Nat. Mater. 2012, 11, 963.

[30] Chen, Z. B.; Cummins, D.; Reinecke, B. N.; Clark, E.; Sunkara, M. K.; Jaramillo, T. F. Nano Lett. 2011, 11, 4168.

[31] Li, M.; Cushing, S. K.; Zhou, X.; Guo, S.; Wu, N. Q. J. Mater. Chem. 2012, 22, 23374.

[32] Wu, N. Q.; Fu, L.; Su, M.; Aslam, M.; Wong, K. C.; Dravid, V. P. Nano Lett. 2004, 4, 383.

[33] Stankovich, S. Piner, R. D.; Chen, X. Q.; Wu, N. Q.; Nguyen, S. T.; Ruoff, R. S. J. Mater. Chem. 2006, 16, 155.

[34] Coehoorn, R.; Haas, C.; Dijstra, J.; Flipse, C.; Degroot, R. A.; Wold, A. Phys. Rev. B. 1987, 35, 6195 .

[35] Wilcoxon, J. P.; Newcomer, P. P.; Samara, G. A. J. Appl. Phys. 1997, 81, 7934.

[36] Zhou, W. J.; Yin, Z. Y.; Du, Y. P.; Huang, X.; Zeng, Z. Y.; Fan, Z. X.; Liu, H.; Wang, J. Y.; Zhang, H. Small 2013, 9, 140.

[37] Gelderman, K.; Lee, L.; Donne, S. W. J. Chem. Educ., 2007, 84, 685. 
[38] Li, X. L.; Wang, H. L.; Robinson, J. T.; Sanchez, H.; Diankov, G.; Dai, H. J. J. Am. Chem. Soc. 2009, 131, 15939.

[39] Tributsch, H.; Bennett, J. C. J. Electroanal. Chem. 1977, 81, 97.

[40] Sivula, K.; Le-Formal, F.; Michael, G. ChemSusChem 2011, 4, 432.

[41] Katz, M. J.; Riha, S. C.; Jeong, N. C.; Martinson, A.; Farha, O. K.; Hupp, J. T. Coord. Chem. Rev. 2012, 256, 2521.

[42] Tilley, S. D.; Cornuz, M.; Sivula, K.; Gratzel, M. Angew. Chem. Int. Ed. 2010, 49, 6405.

[43] Sivula, K.; Zboril, R.; Le Formal, F.; Robert, R.; Weidenkaff, A.; Tucek, J.; Frydrych, J.; Gratzel, M. J. Am. Chem. Soc. 2010, 132, 7436.

[44] Tafalla, D.; Salvador, P.; Benito, R. M. J. Electrochern. Soc. 1990, 137, 1810. 


\section{Charpter 9 Conclusions and Outlook}

\subsection{Conclusions}

The following conclusions are drawn from the investigation:

First, oxygen vacancies play an important role in anatase $\mathrm{TiO}_{2}$ photocatalysis, and more generally in doped photocatalysts. In this study, the anatase $\mathrm{TiO}_{2}$ nanobelts were synthesized by hydrothermal method and followed by reduction in $\mathrm{H}_{2}$ flow at $600{ }^{\circ} \mathrm{C}$ for $3 \mathrm{~h}$ to form oxygen vacancies $\left(0.85\right.$ at. \%) and associated $\mathrm{Ti}^{3+}$ species (3.4 at. \%). Under visible light irradiation, the $3 \mathrm{~d}$ status of $\mathrm{Ti}^{3+}$ acted as a sub-band $\mathrm{in}^{\mathrm{TiO}}{ }_{2}$ band gap, which benefited the separation of electrons and holes and enhanced the light absorption. Thus, the reduced anatase $\mathrm{TiO}_{2}$ nanobelts owned higher photocatalytic activity than that of the pristine anatase $\mathrm{TiO}_{2}$ nanobelts. On the contrary, in UV light, the oxygen vacancies were more apt to act as recombination centers for electrons and holes, which led to less electrons and holes moving to the $\mathrm{TiO}_{2}$ surface. Accordingly, the reduced $\mathrm{TiO}_{2}$ exhibited lower photocatalytic activity than the pristine $\mathrm{TiO}_{2}$. These results showed that if the dopant or reduction process introduces mid-gap or localized states the overall photocatalysis will not improve. Although the visible light performance and absorption will increase, the UV performance will suffer due to increased charge recombination.

Second, perovskite-type layer-structured $\mathrm{La}_{2} \mathrm{Ti}_{2} \mathrm{O}_{7}$ nanosheets were successfully prepared by the hydrothermal method. Nitrogen was doped into perovskite $\mathrm{La}_{2} \mathrm{Ti}_{2} \mathrm{O}_{7}$ lattice by heating $\mathrm{La}_{2} \mathrm{Ti}_{2} \mathrm{O}_{7}$ nanosheets in $\mathrm{NH}_{3}$, which resulted to $0.77 \mathrm{eV}$ of band gap 
narrowing of the $\mathrm{La}_{2} \mathrm{Ti}_{2} \mathrm{O}_{7}$ nanosheets but no change in the particle shape, the dimension and the crystal phase. The nitrogen-doped $\mathrm{La}_{2} \mathrm{Ti}_{2} \mathrm{O}_{7}$ nanosheets showed both enhanced photocatalytic methyl orange degradation in either UV light or visible light. Therefore, the enhancement of photocatalytic activity was originated from the narrowing of the band gap of $\mathrm{La}_{2} \mathrm{Ti}_{2} \mathrm{O}_{7}$ nanosheets. To further increase the photocatalytic water splitting activity for hydrogen generation, Pt nanoparticles (size less than $5 \mathrm{~nm}$ ) were homogeneously dispersed onto the surface of the nitogen-doped $\mathrm{La}_{2} \mathrm{Ti}_{2} \mathrm{O}_{7}$ nanosheets. As a result, the photocatalytic activity of hydrogen generation from water was significantly increased by nitrogen doping and $\mathrm{Pt}$ nanoparticles loading together. These results showed that when the dopant narrows the band gap by introducing a continuum of states by the valence band edge, doping increases performance across the entire spectrum. These provide guidelines to introduce dopants without seeing the decrease in performance measured in Chapter 1.

Third, the $\alpha-\mathrm{Fe}_{2} \mathrm{O}_{3} / \mathrm{rGO}$ composite was synthesized for photocatalytic water splitting to generate oxygen. Our work has proved that rGO can improve performance of the $\mathrm{a}-\mathrm{Fe}_{2} \mathrm{O}_{3}$ for photocatalytic water oxidation, which was due to the following reasons. The suppressed charge recombination and enhanced charge separation in $\alpha-\mathrm{Fe}_{2} \mathrm{O}_{3}$ was realized due to the extraction of photogenerated electrons from $\alpha-\mathrm{Fe}_{2} \mathrm{O}_{3}$ to $\mathrm{rGO}$, which resulted in the enhanced photocatalytic activity toward oxygen evolution. Just as in chapters 3-5 where increased the performance of a large band gap metal oxide photocatalyst was shown by narrowing the band gap, this work showed an equally justified approach is to start with a small band gap metal oxide and increase the charge separation to lead to large enhancements in hydrogen production. 
Fourth, plasmonic Au nanoparticles loaded onto the LTO nanosheets benefit the light absorption efficiency. The plasmonic peak of the Au nanoparticles ranges from 400 to $650 \mathrm{~nm}$. In the plasmon overlaps with the doped NLTO spectrally, leading to a plasmon-induced increase in spectral utilization. Besides, the rGO can improve the charges separation and migration. These combined effects of the surface plasmon of $\mathrm{Au}$ nanoparticles and excellent conductivity of rGO increase the hydrogen generation rate from 44.2 to $163.4 \mu \mathrm{M} \cdot \mathrm{g}^{-1} \cdot \mathrm{h}^{-1}$. The reported results can be used to guide the optimization of the plasmonic effect for photocatalytic $\mathrm{H}_{2}$ generation.

Fifth, we used abundant, low-cost $\mathrm{MoS}_{2}$ and GO to synthesize $p-\mathrm{MoS}_{2} / n$-rGO nanocomposite with nanoscale $p-n$ junctions. GO acted as an efficient substrate to support and inhibit the growth of the $\mathrm{MoS}_{2}$ nanoparticles. By comparing Mott-Schottky results of the $\mathrm{MoS}_{2} / \mathrm{rGO}$ and $p-\mathrm{MoS}_{2} / n-\mathrm{rGO}$, p-n junction was successfully formed. The nanoscale p-n junction can effectively decrease the kinetic energy barrier of the catalytic reaction, facilitate the charge separation and extend the life time of the photo-generated charge carriers. Therefore, the rate of photocatalytic HER of the $\mathrm{MoS}_{2} / \mathrm{rGO}$ nanocomposite in simulated solar light was sgnificantly increased from $8.4 \mu \mathrm{M} \mathrm{g}^{-1} \mathrm{~h}^{-1}$ to $22.5 \mu \mathrm{M} \mathrm{g}^{-1} \mathrm{~h}^{-1}$ by doping nitrogen into rGO. The results of these section further improve upon the enhancements of the previous chapters by replacing the expensive Pt co-catalyst with an earth abundant material. This is important, otherwise the effects of manufacturing renewable energy materials would offset the clean energy the produce. This chapter showed that $\mathrm{MoS}_{2}$ is not only a earth-friendly alternative to $\mathrm{Pt}$, but that the performance of the device can actually be further improved by this technique. 
Overall this thesis has developed strategies for the enhancement of hydrogen generation in both large and small band gap metal oxide photocatalysts, as well as showing an earth-friendly co-catalyst to replace noble metals. The information gained through these studies allows a large flexibility in materials selection when designing hydrogen generation devices without any sacrifice in performance or eco-friendliness.

\subsection{Outlook}

Following this thesis, I recommend future research should be focused on the following areas:

First, deeper investigations should be conducted on the mechanisms of the effect of the oxygen vacancies to the anatase $\mathrm{TiO}_{2}$. What is the exact position of the $\mathrm{Ti}^{3+}$ orbital? How does the $\mathrm{Ti}^{3+}$ affect the charge recombination? Does the crystal distortion affect the charge mobility and charge separation in the anatase $\mathrm{TiO}_{2}$ ? In particular, does the oxygen vacancy effect the other metal oxide semiconductors the same as $\mathrm{TiO}_{2}$ ?

Second, since nitrogen doping can significantly increase the light absorption of the $\mathrm{La}_{2} \mathrm{Ti}_{2} \mathrm{O}_{7}$ nanosheets, what is the relationship between the doped nitrogen and other elements in the $\mathrm{La}_{2} \mathrm{Ti}_{2} \mathrm{O}_{7}$ nanosheets? Does the position of Pt loading onto the $\mathrm{La}_{2} \mathrm{Ti}_{2} \mathrm{O}_{7}$ nanosheets (plate surfaces or the edges of the $\mathrm{La}_{2} \mathrm{Ti}_{2} \mathrm{O}_{7}$ nanosheets) affect the photocatalytic $\mathrm{H}_{2}$ generation performance?

Third, the $\mathrm{MoS}_{2}$ has a layered structure. In the composite of $\mathrm{MoS}_{2} / \mathrm{rGO}$, the $\mathrm{MoS}_{2}$ shown in the HRTEM had several layers, so how does the number of the $\mathrm{MoS}_{2}$ layers 
affect the photocatalytic $\mathrm{H}_{2}$ generation? Will a one-layer $\mathrm{MoS}_{2} / \mathrm{rGO}$ composite have the best photocatalytic performance?

Fourth, targeting to solve the global energy consumption, the photocatalytic $\mathrm{H}_{2}$ generation must be improved at an industrial scale. Therefore, all the nanostructure materials studied in this thesis should be studied for their long-term catalytic stability, scaleup tests etc.. The photocatalytic instruments should also be re-designed to be favorable industrially. 


\section{List of Publications}

1. Solar Hydrogen Generation by Nanoscale p-n Junction of p-type Molybdenum Disulfide/n-type Nitrogen-Doped Reduced Graphene Oxide, F. K. Meng, J. T. Li, S. K. Cushing, M. J. Zhi, N. Q. Wu, Journal of the American Chemical Society, 135 (2013), 10286-10289.

2. Photocatalytic Water Oxidation by Hematite/Reduced Graphene Oxide Composite, F. K. Meng, J. T. Li, S. K. Cushing, J. Bright, M. J. Zhi, J. D. Rowley, Z. L. Hong, A. Manivannan, A. D. Bristow, N. Q. Wu, ACS Catalysis, 3 (2013), 746-751.

3. Visible-light photocatalytic activity of nitrogen-doped $\mathrm{La}_{2} \mathrm{Ti}_{2} \mathrm{O}_{7}$ nanosheets originating from band gap narrowing, F. K. Meng, Z. Hong, J. Arndt, M. Li, M. Zhi, F. Yang, N. Q. Wu, Nano Research, 5 (2012), 213-221.

4. Photocatalytic generation of hydrogen with visible-light nitrogen-doped lanthanum titanium oxides, F. K. Meng, J. Li, Z. Hong, A. Sakla, N. Q. Wu, Catalysis Today, 199 (2013), 48-52.

5. Photocatalytic activity enhanced by plasmonic resonant energy transfer from metal to semiconductor, S. K. Cushing, J. Li, F. K. Meng, T. Senty, S. Suri, M. Zhi, M. Li, A. Bristow, N. Q. Wu, Journal of the American Chemical Society, 134 (2012), 1503315041.

6. Differential mouse pulmonary dose- and time course-responses to titanium dioxide nanospheres and nanobelts, D. W. Porter, N. Q. Wu, A. F. Hubbs, R. R. Mercer, K. Funk, F. K. Meng, J. Li, M. G. Wolfarth, L. Battelli, S. Friend, M. Andrew, R. 
Hamilton, K. Sriram, F. Yang, V. Castranova, and A. Holian, Toxicological Sciences, 131 (2013), 179-193.

7. Photoelectrochemical performance enhanced by nickel oxide-hematite $p-n$ junction photoanode, J. Li, F. K. Meng, S. Suri, W. Ding, F. Huang, N. Q. Wu, Chemical Communications, 48 (2012), 8213-8215.

8. Highly conductive electrospun carbon nanofiber/ $\mathrm{MnO}_{2}$ coaxial nano-cables for high energy and power density supercapacitors, M. Zhi, A. Manivannan, F. K. Meng, N. Q. Wu, Journal of Power Sources, 208 (2012), 345-353.

9. Ag@Cu2O Core-Shell Nanoparticles as Visible-Light Plasmonic Photocatalysts, J. T. Li, S. K. Cushing, J. Bright, F. K. Meng, T. R. Senty, P. Zheng, A. D. Bristow, ACS Catalysis, 3 (2013), 47-51.

10. Plasmon-induced Photonic and Energy-transfer Enhancement of Solar Water Splitting by a Hematite Nanorod Array, J. T. Li, S. K. Cushing, P. Zheng, F. K. Meng, D. Chu, N. Q. Wu, Nature Communications, (2013), doi: 10.1038/ncomms3651.

11. A Systematic Study of the Catalytic Behavior at Enzyme-metal-oxide Nanointerfaces, A. S. Campbell, C. B. Dong, A. Maloney, J. Hardinger, X. Hu, F. K. Meng, N. Q. Wu, C. Dinu, Nano Life, (2013), accepted. 